\title{
OSCILLATORY DYNAMICS OF THE ACTIN CYTOSKELETON
}

\author{
Dissertation \\ zur Erlangung des mathematisch-naturwissenschaftlichen \\ Doktorgrades \\ „,Doctor rerum naturalium” \\ der Georg-August-Universität Göttingen
}

vorgelegt von

Christian WESTENDORF

aus Rostock, Deutschland

Göttingen, 2012 
Referent: Prof. Dr. Eberhard Bodenschatz

Korreferent: Prof. Dr. Oliver STEINBOCK

Tag der mündlichen Prüfung: 28.11.2012 
1. Introduction 12

1.1. Dictyostelium discoideum - a model organism . . . . . . . . . . . . . . . 13

1.2. The actin cytoskeleton and its in vivo observation . . . . . . . . . . 16

1.3. The Dictyostelium discoideum chemotactic signal processing system . . . . 19

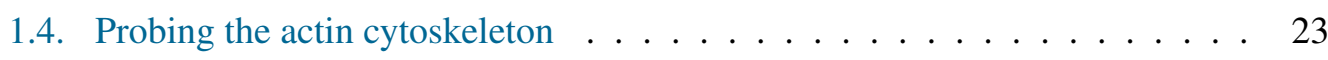

1.5. Linear and non-linear systems . . . . . . . . . . . . . . 26

2. Material and methods $\quad 29$

2.1. Cell culture . . . . . . . . . . . . . . . . . . . . . . . . . . . . . . 29

2.2. Microfluidics . . . . . . . . . . . . . . . . . . 30

2.3. Experimental setup and its characterization . . . . . . . . . 33

2.4. Image processing . . . . . . . . . . . . . . . . . . . . . . . . . . . . . . . . . . . . . . . .

2.5. Signal processing . . . . . . . . . . . . . . . . 42

3. Actin cytoskeletal response to short-time stimuli 44

3.1. Observed temporal patterns . . . . . . . . . . . . . . . . . . . 47

3.2. Characterization of the periodic response . . . . . . . . . . . . . 48

3.3. Nonlinear regimes in the actin cytoskeleton . . . . . . . . . . 58

3.4. Theoretical approach . . . . . . . . . . . . . . 61 
3.5. Discussion . . . . . . . . . . . . . . . . . . 68

3.6. Chemotactic response to short-time stimuli f . . . . . . . . . . 72

4. On the existence of self-sustained cytoskeletal oscillations $\quad \mathbf{8 0}$

4.1. Self-sustained actin oscillations in SCAR(-)/PIR121(-) LimE-GFP . . . . . 81

4.2. Dependence of the cortical actin oscillations on different conditions . . . . 83

4.3. Non-optical detection of self-sustained oscillations . . . . . . . . . . . 87

4.4. Periodic stimulation of the SCAR(-)/PIR121(-) knockout . . . . . . . . . 90

4.5. Discussion . . . . . . . . . . . . . . . . . . . . . 92

5. Eukaryotic cell flattening 95

5.1. Overlay techniques . . . . . . . . . . . . . . . . . . 96

5.2. Microfluidic approaches . . . . . . . . . . . . . . . . . 98

5.3. Discussion . . . . . . . . . . . . . . . . . . . . . . . 104

5.4. Applications of the cell flattening devices . . . . . . . . . . . . . 107

$\begin{array}{lr}\text { Conclusion } & 110\end{array}$

$\begin{array}{lr}\text { Acknowledgement } & 112\end{array}$

$\begin{array}{ll}\text { Appendix } & \mathbf{1 1 6}\end{array}$

A. Supplementary material on CD-ROM . . . . . . . . . . . . . . 116

B. MATLAB script for DDE simulation . . . . . . . . . . . . . . . 117

$\begin{array}{lr}\text { Bibliography } & 120\end{array}$

$\begin{array}{lr}\text { Curriculum vitae } & 140\end{array}$ 
1.1. Life cycle of Dictyostelium discoideum . . . . . . . . . . . . . . . 15

1.2. The actin cytoskeleton . . . . . . . . . . . . . . . 17

1.3. Chemotaxis in Dictyostelium discoideum . . . . . . . . . . . . . . 20

1.4. Chemistry of DMNB-caged compounds . . . . . . . . . . . . . 24

1.5. Principle of flow photolysis . . . . . . . . . . . . . . 25

1.6. Characterization of a signal processing system . . . . . . . . . 28

2.1. Soft lithography and replica molding . . . . . . . . . . . . . . . . . . . . . . . . . 32

2.2. Channel geometries . . . . . . . . . . . . . . . . . . 33

2.3. Characterization of the FV1000 confocal laser scanning system . . . . . . . 34

2.4. Average flow speed measurement . . . . . . . . . . . . . . . . 35

2.5. Measured and calculated concentration profiles after uncaging . . . . . . 37

2.6. Microfluidic setup for periodic stimulation experiments . . . . . . . . . . . 39

2.7. Image segmentation $\ldots \ldots \ldots$. . . . . . . . . . . . . 41

3.1. Response behavior of the filamentous actin . . . . . . . . . . . . . 46

3.2. Variety of the observed oscillatory patterns . . . . . . . . . . . . . . . . . . . . . . . 48

3.3. Periodic stimulation at low external forcing . . . . . . . . . . . . 50

3.4. Periodic stimulation at medium periodic forcing . . . . . . . . . . . 52

3.5. Periodic stimulation at high periodic forcing . . . . . . . . . . . . 54

3.6. Characterization of the oscillatory properties . . . . . . . . . . . . 56

3.7. Characterization of the intrinsic timescale . . . . . . . . . . . . . 57

3.8. Superposition of the pulse responses . . . . . . . . . . . . . . 60 
3.9. Properties of the self sustained oscillations . . . . . . . . . . . . . . 62

3.10. Depolymerization time delay . . . . . . . . . . . . . . . . . 64

3.11. Pulse and step response of the DDE model . . . . . . . . . . . . . . . 66

3.12. Response of the DDE model to periodic perturbation . . . . . . . . . . 67

3.13. Periodic stimulation of other chemotaxis of cytoskeletal markers . . . . . 71

3.14. Chemotactic response to pulse and step input functions . . . . . . . . 73

3.15. Chemotactic response to different periodic input functions . . . . . . . 75

3.16. Chemotactic response control . . . . . . . . . . . . . . 76

3.17. Averaged velocity over time _ . . . . . . . . . . . . . 78

3.18. Chemotactic response to an additional gradient . . . . . . . . . . 79

4.1. Model of the SCAR-complex . . . . . . . . . . . . . . . . . . . . . 82

4.2. Influence of the laser intensity on the autonomous oscillations . . . . . . . 84

4.3. Phase analysis of temporarily interupted oscillations . . . . . . . . . . . 86

4.4. Spatial dependence of the autonomous oscillations . . . . . . . . . . . 87

4.5. Atomic force microscopy on the SCAR(-)/PIR121(-) double knockout . . . 89

4.6. Periodic forcing of the SCAR(-)/PIR121(-) double knockout . . . . . . . 90

4.7. Comparison of the wildtype and the mutant response to periodic forcing . . 91

4.8. Attractor reconstruction . . . . . . . . . . . . . . . . . . . . 93

5.1. Oil overlay technique . . . . . . . . . . . . . . . . . . . . . 97

5.2. Cell flattening with overlay techniques . . . . . . . . . . . . . . 97

5.3. Single-layer microfluidic device . . . . . . . . . . . . . . . . . . . . 99

5.4. Characterization of the single-layer microfluidic device . . . . . . . . . 100

5.5. Microfluidic through-flow actuator . . . . . . . . . . . . . . . 102

5.6. Characterization of the microfluidic through-flow actuator . . . . . . 103

5.7. Exemplary micrographs visualizing the cell flattening . . . . . . . . . 105

5.8. Example analysis of a flattened Dictyostelium cell . . . . . . . . . . . . 109 


\section{List of Tables}

2.1. List of all used Dictyostelium discoideum mutants . . . . . . . . . . . . . 30

5.1. Comparison of the devices for eukaryotic cell flattening . . . . . . . . . . 106 
ACV

Aip1

AFM

ADP

Arp

ATP

CAMP

CAR

CEASAR

CLSM

DDE

D. discoideum

DMNB

DMSO

ECIS

F-actin

FEM

FFT

G-actin

GFP

GTP

HPLC

MPI BPC
Autocovariance

Actin-interacting protein 1

Atomic force microscopy

Adenosine-5'-diphosphate

Actin related protein

Adenosine-5'-triphosphate

3'-5' cyclic adenosine monophosphate

cAMP receptor

Center of Advanced European Studies and Research

Confocal laser scanning microscopy

Delay differential equation

Dictyostelium discoideum

4,5-dimethoxy-2-nitrobenzyl

Dimethyl sulfoxide

Electrical cell substrate impedance sensing

Filamentous actin

Finite element method

Fast Fourier transform

Monomeric globular actin

Green fluorescent protein

Guanosine-5'-triphosphate

High performance liquid chromatography

Max Planck Institute for Biophysical Chemistry 


$\begin{array}{ll}\text { MPI DS } & \text { Max Planck Institute for Dynamics and Self-Organization } \\ \text { MS } & \text { Mass spectrometry } \\ \text { PB } & \text { Phosphate buffer } \\ \text { PDMS } & \text { Polydimethylsiloxane } \\ \text { PI3K } & \text { Phospho-inositol-3-kinase } \\ \text { PIP2 } & \text { Phosphatidylinositol 4,5-bisphosphate } \\ \text { PIP3 } & \text { Phosphatidylinositol 3,4,5-trisphosphate } \\ \text { PLA2 } & \text { Phospholipase A2 } \\ \text { PTEN } & \text { Phosphatase and tensin homolog } \\ \text { RFP } & \text { Red fluorescent protein } \\ \text { SCAR } & \text { Suppressor of cAMP receptor mutation } \\ \text { SGC } & \text { Soluble Guanylyl-cyclase } \\ \text { TIRF } & \text { Total internal reflection fluorescence } \\ \text { WASP } & \text { Wiskott-Aldrich syndrome protein }\end{array}$


Abstract

The actin cytoskeleton is ubiquitously distributed among eukaryotic organisms and determines the shape and the movement of eukaryotic cells. In single migratory cells, rapid and localized actin polymerization often follows an external stimulus, engaging migration towards or away from the stimulus. This process is termed chemotaxis and plays a crucial role in several different eukaryotic processes, for instance the inflammatory response of neutrophils and macrophages, cancer metastasis or the growth of axons. A suitable model system to study the chemotactically induced response of the actin cytoskeleton is the social amoeba Dictyostelium discoideum. Within its life cycle, it exists as an autonomously living and proliferating single cell as well as a differentiated and multicellular organism. The developmental program to multicellularity is engaged under starvation conditions and serves as a survival mechanism. Within this development, the cells become chemotactic towards 3'-5' cyclic adenosine monophosphate (cAMP), which is emitted spontaneously by cells to attract other amoebae. If a $D$. discoideum amoeba is exposed to a sudden upshift of external cAMP concentration, the actin cytoskeleton responds biphasically, i.e. an initial sharp increase in filamentous actin concentration is followed by a broader and smaller maximum. This biphasic behavior resembles a damped oscillation, if the input is shortened to a brief pulse of cAMP.

Within this thesis, the oscillatory properties of the actin cytoskeleton and the chemotactic signaling cascade, controlling the actin polymerization were probed by different periodic input functions of externally administered cAMP. The pulses were created using the flow photolysis method, in which chemically caged molecules are photochemically released in the microfluidic flow. Pulse lengths down to $1.5 \mathrm{~s}$ are possible with concentration switching times below $1 \mathrm{~s}$. The response of the $D$. discoideum actin cytoskeleton, which was 
labeled via LimE-GFP (a protein specifically associated with filamentous actin) suggests a resonance of the actin cytoskeleton at input periods equal to $20 \mathrm{~s}$. The second harmonic frequency becomes apparent, above the resonance timescale. Furthermore, the response to short periods suggest an onset of oscillatory behavior, i.e. the actin cytoskeleton follows the external forcing, above input periods of $8 \mathrm{~s}$. This is considerably shorter than the $20 \mathrm{~s}$ timescale, previously estimated for the chemotactic signaling cascade. In conjunction with these observations and the observation that a minor fraction of all cells showed selfsustained oscillations of the LimE-GFP fluorescence intensity, we proposed that the actin cytoskeleton and/or the signal processing cascade operate close to an oscillatory instability. A delay differential equation, which shows this type of Hopf bifurcation was successfully used to model the principal observed temporal patterns. The delay was experimentally verified by measuring the delay between LimE-GFP, the polymerization marker and CoroninGFP as well as Aip1-GFP, two labels of the actin depolymerization process.

Interestingly self-sustained oscillations of the LimE-GFP label have been reported previously in a $D$. discoideum knockout mutant of the Arp2/3 regulatory SCAR-complex. One could speculate that, within this mutant, a larger fraction of cells passed the instability criterion, explaining the higher rate of observed oscillations. The oscillatory patterns, obtained by confocal laser scanning microscopy (CLSM), lacked verification by other microscopy techniques and a possible light dependence of the oscillations was supposed. Additional CLSM experiments were conducted within this thesis and such light dependence was not observed. However, it was verified that the oscillations occur globally within the cell. Assuming that the constant polymerization and depolymerization cycles act directly on the cell membrane gave rise to the possibility to read out the height of a cell as a non-optical parameter. Atomic force microscopy (AFM) height measurements over time characterized the difference as stronger fluctuations of the cell height within the SCAR(-)/PIR121(-) knockout mutant.

To study the actin cytoskeleton on the timescales of polymerizing filamentous actin bundles other microscopy techniques than CLSM need to be applied. Most prominently the total internal reflection fluorescence microscopy (TIRF) images solely the lower boundary of the specimen on a high time resolution. One way to improve the quality of TIRF imaging is to compress the cell and therefore to confine its lower membrane to the glass surface, increasing the observable parts of the cell. Previous approaches involved overlaying techniques, which lack precise control and stability. Here, easy-to-handle microfluidic-based flattening techniques were developed and characterized. Microfluidic compression devices greatly improved the degree of control over flattening and duration of the experiments, but are nevertheless generable within one to two hours. 
CHAPTER 1

Introduction

Living organisms are dynamic and adaptive systems, which rely on a constant throughput of energy (Maroto and Monak, 2008). On a single cell level, oscillations are representations of the cells dynamic properties (Kruse and Juelicher, 2005) and emerge from collective behavior. One distinguishes transient and self-sustained type of oscillations. The transient oscillation follows a sudden state change of the system and decays quickly back to it. In phase space, this is represented by a trajectory excited from the fixed point and then spiraling back to it. If the limit cycle is stable, one speaks of a self-sustained oscillation (Strogatz, 2000). The system continuously drains energy from a source to maintain the oscillation. Perhaps the most famous example of self-sustained oscillations are the circadian rhythms (Huang et al., 2011). Examples from cell biology range from the oscillations during the bacterial cell division (Hu and Lutkenhaus, 1999) to the oscillations of the auditory hair bundles (see for instance Martin et al. (2003)). Especially the eukaryotic actin cytoskeleton gathered attention in the context of such dynamical systems; its fast polymerization and depolymerization kinetics give cells the possibility to quickly change their shape, move and adapt to varying environments.

Within this work, the oscillatory properties of the actin cytoskeleton have been probed by application of external input functions. As a suitable experimental system to study the actin cytoskeleton, the model organism Dictyostelium discoideum was employed. 


\subsection{Dictyostelium discoideum - a model organism}

The first description of the Dictyostelium genus dates back to the year 1869 by Oscar Brefeld $^{1}$. According to Kessin (2001) he chose the term Dicty, which means 'net-like' and stelium, referring to 'tower' as a reference to the interesting life cycle of this organism. Dictyostelium discoideum was first isolated in the 1930's from forest soil (Raper, 1935) and, until nowadays, it gathered the attention of both biologists and physicists for various reasons. Within its life cycle, it exists in both forms: as an isolated amoeba and as a differentiated multicellular organism. Therefore, it has become popular to call $D$. discoideum a social amoeba. However, it is classified as a cellular slime mold. Slime molds are eukaryotic and heterotrophic organisms, but are not anymore considered to be part of the fungi kingdom. Instead they are included in the phylum amoebozoa, part of the protists (Eichinger et al., 2005). D. discoideum feeds on a variety of bacteria via phagocytosis (Raper (1937), Raper and Smith (1939), Depraitere and Darmon (1978)). As long as food is available in the nearest neighborhood, $D$. discoideum lives as a solitary amoeba, about $10 \mu \mathrm{m}$ in size, and proliferates by cell division with a division rate of approximately $4 \mathrm{~h}$ (Kessin, 2001). In figure 1.1A a sketch of the entire lifecycle is shown. The solitary living and proliferating stage is designated as the vegetative cycle.

If the environment is depleted of nutrients a developmental program towards a multicellular organism turns on, referred to as the social cycle. We can think of this as a survival mechanism, in which the organism in its multicellular stage can overcome larger distances and finally develops into a fruiting body capable of dispersing spores (Chisholm and Firtel, 2004). The developing $D$. discoideum cells become sensitive to 3'-5'-cyclic adenosine monophosphate (cAMP). Spontaneously, cells start to produce and emit cAMP (Gerisch and Wick (1975), Tomchik and Devreotes (1981)). Neighboring cells receive this signal and themselves start to produce and to emit cAMP. This is controlled by an internal relay (Laub and Loomis, 1998), within the cell, which first becomes stimulated, then emits cAMP and enters a transient refractory period in which it remains quiescent (the entire relay takes about 6 minutes, depending on the developmental state of the cell). This relay process is responsible for the observed travelling waves of cAMP through the population of amoebae, which can be described in the context of excitable media (Martiel and Goldbeter, 1987). The developed $D$. discoideum cells are chemotactic towards cAMP and, through several passing cAMP waves, the cells come into close contact by chemotaxis. Finally these cells build streams of cells moving towards one aggregation center. The aggregating cells build the mound stage and differentiate into prestalk and prespore cells (Chisholm and Firtel (2004),

${ }^{1}$ The first description of Dictyostelium was on the species Dictyostelium mucoroides. 
Gomer and Firtel (1987)). The cells sort out with the prestalk cells moving to the middle and the prespore cells migrating towards the outside. The mound develops into the slug state, which itself is migratory and both photo- and chemotactic (Fisher et al., 1981) (see figure 1.1A,B roman III). After cessation of the slug, the culmination starts and leads to a fruiting body, where the stalk cells carry the spore cells on top (see figure 1.1A,B roman V). The spores are released, eventually spread, and finally reach the amoeba stage again, while the stalk cells die. Note that throughout the entire cycle the cellularity remains, i.e. the cells may develop and differentiate, but do not loose their integrity. Therefore $D$. discoideum and the other members of this genus are termed cellular slime mold to distinguish it from the acellular slime molds with Physarum polycephalon being the most famous in current science (see for instance Tero et al. (2010)).

Throughout the vegetative and the social cycle $D$. discoideum remains an haploid organism and no genetic material is exchanged or recombined. Sexual reproduction occurs under very distinct environmental conditions via the attraction of different mating types, consequent fusion to the diploid stage and formation of macrocysts (figure 1.1A, sexual cycle).

All experiments carried out in this thesis required starved and partially developed chemotactic $D$. discoideum cells. We should bear in mind that experiments carried out during this chemotactic stage are time limited. The $D$. discoideum amoeba constantly develops. Due to this, the genetic expression pattern is subject to constant changes (Parent and Devreotes, 1996). For example, the expression of membrane bound cAMP receptors start just 4 hours after starvation and lasts until 10 hours after the onset of starvation. The maximal chemotactic motility is reached after 7 to 8 hours. This sets the phase of the development, in which chemotaxis experiments can be carried out on 7 - 10 hours starved $D$. discoideum amoebae. In the early 1960's efforts were undertaken to maintain Dictyostelium in a bacteria free so called axenic culture (Sussman and Sussman (1967), Ashworth and Watts (1970), Franke and Kessin (1977)). The selection of Dictyostelium resulted in strains usually abbreviated by $\mathrm{AX}$ and an additional number, which designate their origin. These axenic strains are the set of mutated $D$. discoideum strains which can feed on macropinocytosis (Hacker et al. (1997), Williams et al. (1974)), i.e. the fluid uptake from the surrounding medium by endocytosis. Axenic strains greatly improved the handleability of Dictyostelium as a lab organism and a variety of different strains are available (Kreppel et al., 2004). In this thesis, different mutants introduced in the AX2 (Sussman and Sussman, 1967) and AX3 (Loomis, 1971) background have been used. We should always keep in mind that the strains originate from different parental cell lines (these parental cell lines are the natural isolates of D. discoideum) and that different mutagenesis methods had been used to create them. 

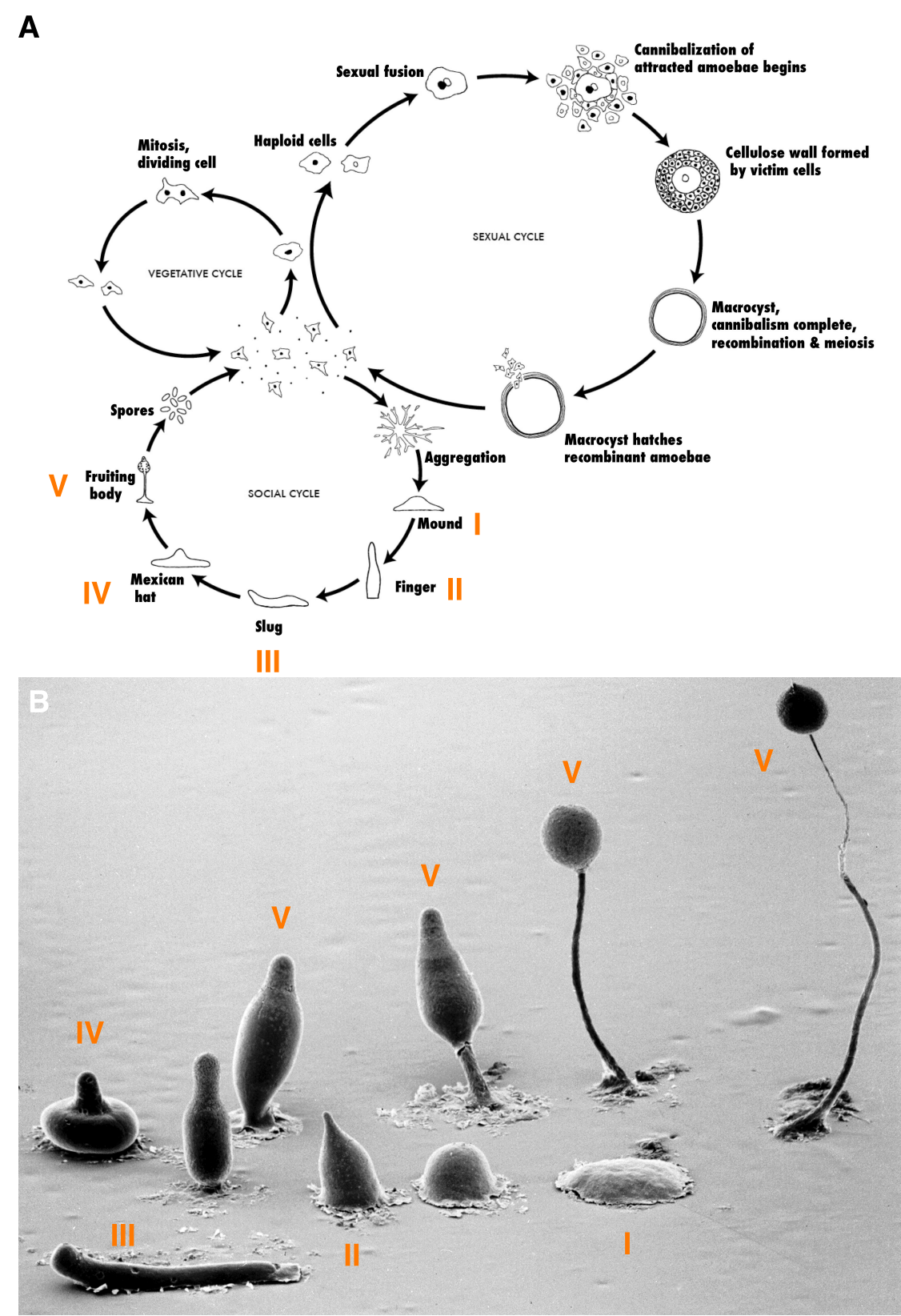

Figure 1.1.: The life cycle of Dictyostelium discoideum. (A) Sketch of the D. discoideum life cycle, which can be subdivided into three subcycles. (1) As long as food is not limiting, the amoebae life solitary, feed on bacteria and proliferate (vegetative cycle). (2) Under starvation conditions the development into a multicellular organism is engaged. This is part of a survival program, finally resulting in the production and release of spores (social cycle). (3) Within these two cycles the cells remain haploid. An exchange of genetic material is possible via the attraction of different mating types and consequent fusion of them (sexual cycle). Further information are given in the text. (CD. Brown and J.E. Strassmann. Permission granted by the Creative Commons Attribution. (B) Collage of different developmental stages of the $D$. discoideum within the social cycle. The roman numbers indicate the different stages labelled in (A). Several stages of the fruiting body are shown (V). (C)M.J. Grimson and R.L. Blanton, Biological Sciences and Electron Microscopy Laboratory, Texas Tech University. Reprint permission granted by the authors. 


\subsection{The actin cytoskeleton and its in vivo observation}

\subsubsection{The actin cytoskeleton}

The actin cytoskeleton is a key to understanding the dynamics of the living cell. It is one part of the eukaryotic cytoskeleton, the others being the microtubuli and the intermediate filaments. It ranges among the most abundant proteins on earth (Pollard and Cooper, 2009) and exists in two states: (1) the monomeric form, termed globular or G-actin and (2) the polymerized form, termed filamentous or F-actin. Polymerization of the actin filament from the monomer can occur spontaneously, but is energetically unfavoured (see figure 1.2B). At the trimer stage, the polymerization of the ATP bond monomeric actin molecules becomes exothermic and proceeds faster. The hydrolyzation of the ATP molecule into ADP and inorganic phosphate occurs shortly after the polymerization and is not necessary for the polymerization process itself, but gives a polarization to the actin molecule. The newest monomers, i.e. in the freshly polymerized part of the actin filament, still are bonded to the ATP molecule and are designated as the barbed end of the filament. The dissociation rate for the ADP-containing pointed end of the F-actin filament surpasses the association rate of new monomers. Therefore net depolymerization dominates at the pointed end and the filament moves forward (Pollard and Borisy, 2003). This process is known as treadmilling and is intrinsically a slow process. More than 100 proteins are known to be associated with the actin cytoskeleton (see Eichinger et al. (2005) for the D. discoideum genome project), which enhance the polymerization. These proteins are known as the actin nucleators (Machesky and Insall, 1998). The nucleation onto the filament or other monomeric actin molecules is mediated via the Formins (sketched in figure 1.2C-D) (Evangelista et al. (2003), Goode and Eck (2007)). A key player in the formation of the actin network is the Arp2/3-complex, consisting of 7 members, with two proteins being closely related to the actin monomer (actin-related proteins) (Pollard (2007), Goley and Welch (2006)). The Arp2/3-complex binds to an actin filament and initiates the growth of a new daughter filament at an particular angle (sketched in figure 1.2E)(Welch and Mullins, 2002). It has intrinsically only a weak nucleation activity but increases its activity upon binding of different regulators, called the nucleation promoting factors. These are namely the suppressor of cAMP receptor mutation and Wiskott-Aldrich syndrome protein (WASP) family verprolin homology protein (SCAR/WAVE) ${ }^{2}$ and the WASP families of proteins. Here of particular interest is the SCAR complex (see chapter 4 for details on its biochemistry), which couples external signals to the actin cytoskeleton via the Arp2/3-complex (see figure 1.2E).

\footnotetext{
${ }^{2}$ In the remainder of the text, we will use only SCAR to designate this protein.
} 
Rapid polymerization and branching of the filamentous actin network is a transient process. A turnover of the filamentous actin is necessary to maintain a pool of monomeric actin molecules. The capping of a particular barbed filament end prevents it from further polymerization and severing, as well as depolymerization disassemble it. These processes are also tightly regulated by numerous proteins. Two of them, Coronin and Aip1, are of particular interest within this work. Coronin was described as being associated with F-actin and is known to bind to and to inhibit the action of the Arp2/3 complex (DeHostos et al, 1991). The filament disassembly is driven by the conserved family of ADF/Cofilins (Pollard et al., 2000). Next to Coronin, also the Actin-interacting protein 1 (Aip1) binds to the $\mathrm{ADF} / \mathrm{Cofilin}$ and aids in the disassembly of the actin filaments (Okada et al. (1999)). Both proteins labeled with a GFP-tag can serve as markers for the depolymerization process. The released monomeric and ADP actin molecules are bound by Profilin (Carlsson et al., 1977), which catalyses the exchange of ADP to ATP and the cycle is completed (figure 1.2C).
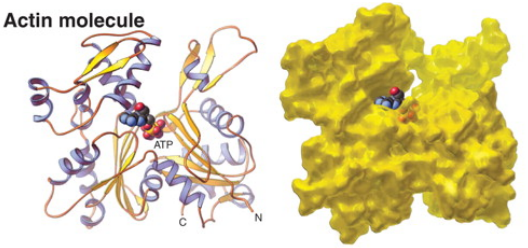

B Spontaneous nucleation and elongation

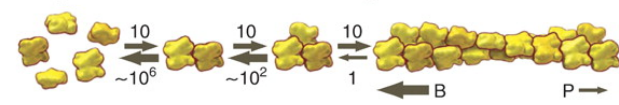

C

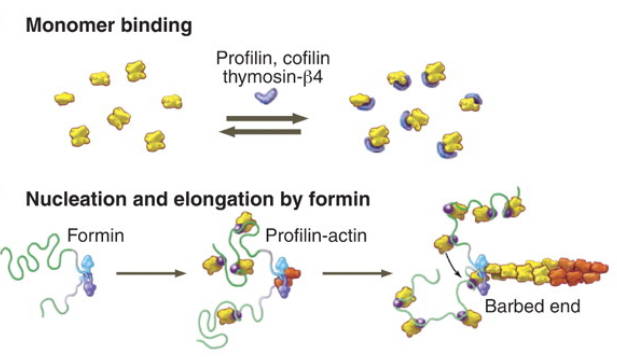

E Branching nucleation by Arp2/3 complex

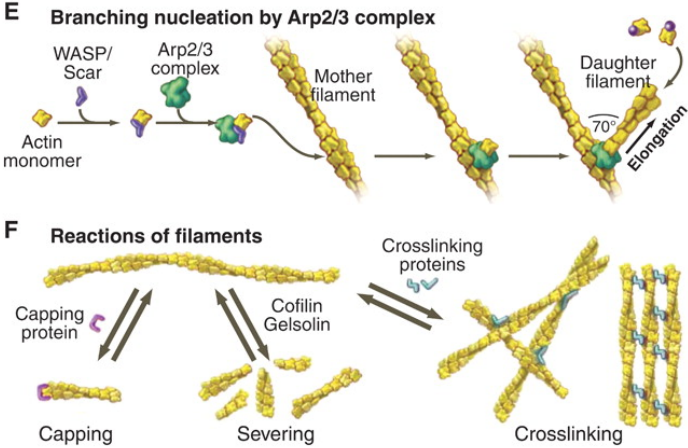

G Myosin-V walking on an actin filament

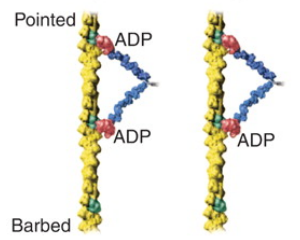

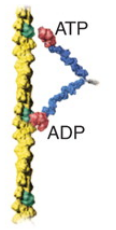

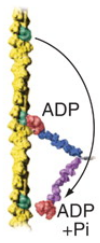

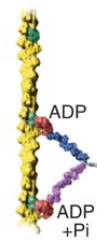

Figure 1.2.: Fundamental structures and reactions of the actin cytoskeleton. (A) Globular structure of the actin molecule. (B) Spontaneous nucleation and elongation of the filamentous actin molecule. The primary nucleation and the extension to a trimer is energetically unfavoured. (C-D) Action of the actin nucleation and elongation factors profilin and formin. (E) Branching and consequent forming of new daughter filaments, by the nucleation factor Arp2/3. This complex requires activation by the SCAR complex prior to binding the F-Actin. (F) The formed filaments can be blocked from further elongation by capping proteins, disassembled by severing proteins or crosslinked to obtain networks and/or bundles of F-Actin. (G) The motor protein myosin moves on the actin filament by hydrolyzation of ATP. From Pollard and Cooper (2009), which is itself redrawn from Pollard (2007). Reprinted with permission from AAAS. 
This cycle describes the steady-state dynamics of the actin cytoskeleton. However localized actin polymerization can be induced by external stimuli. This requires that the spatial information of the stimulus is translated into the intracellular biochemistry (see section 1.3). The dynamics of such stimulated actin polymerization now includes the dynamics of the upstream signalling pathway and of the actin cytoskeleton. Localized actin polymerization gives rise to pseudopods, but the actin cytoskeleton also builds up the shape of an amoeboid cell. A dense layer of F-actin below the cell membrane, which is usually assigned as the cell cortex, stabilizes the cell shape. However, also this cortex is not necessarily stable and so called actin waves have been observed in the absence of external stimuli (Vicker (2002), Bretschneider et al. (2004)). These are waves of high filamentous actin concentration travelling along the cell boundary and display a self-organizing behavior of the actin cytoskeleton. It has been suggested that these waves are not an artificially introduced phenomenon ${ }^{3}$, but in fact are related to processes like pseudopod extension (Bretschneider et al., 2009).

\subsubsection{Filamentous actin labels}

Fluorescence microscopy-based experimental observation of filamentous actin requires labeling of the actin molecules. Prior to genetic engineering, the cell actin cytoskeleton was labeled with fluorescent analogues of the toxin Phalloidin, which binds the filamentous actin and prevents it from depolymerization ${ }^{4}$, imposing the death of the investigated cell (Cooper, 1987). With the available green fluorescent protein (GFP, Tsien (1998)) and the newly developed genetic tools one aimed to find proteins suitable to be tagged with GFP (or other fluorescent proteins). The straightforward labeling of the actin protein itself (Act15 in $D$. discoideum) led to disappointing results. The GFP tag actually changes the polymerization kinetics of the actin cytoskeleton (Pang et al., 1998). As early as 1995, Gerisch and co-workers searched for actin-associated proteins suitable for in vivo imaging of the filamentous actin network. Coronin, as described above is part of the regulation of the actin-severing process. It therefore localizes with older parts of the filamentous actin molecule and Coronin-GFP was succesfully applied to label F-actin (Gerisch et al., 1995). Further screening of actin-associated proteins led to the discovery of DdLim (later renamed DdLimE), which is a LIM-domain containing protein. The LIM-domain, first found in homeodomain proteins ${ }^{5}$, contains two cystein-rich zinc finger motifs, a glycine-rich domain and a coiled-coil domain (Freyd et al. (1990), Sadler et al. (1992)). In D. discoideum,

\footnotetext{
${ }^{3}$ The initial observation of actin waves required the addition of drugs, such as Latrunculin.

${ }^{4}$ The toxicity of Phalloidin is based on the affinity to the actin cytoskeleton.

${ }^{5}$ Homeodomain proteins are transcription factors often associated with the organism development and morphogenesis.
} 
LimE is involved in the cytokinesis, as overexpression and knockout of its gene lead to severe cytokinesis defects (Prassler et al. (1998), Schneider et al. (2003)). Comparing the LimE-GFP fluorescence profile with a Phalloidin staining ${ }^{6}$ revealed that the overlay is about 97\%. Furthermore, LimE-GFP associates with micropinosomes, i.e. small vesicle like inclusions of fluids. The LimE protein was shown to co-localize with the Ras-GTPase Rac1A (Prassler et al. (1998) and recently Filic et al. (2012)). This is a direct activator of the SCAR complex and might explain the affinity of LimE-GFP to the actin cytoskeleton.

In the subsequent years, different LIM domain containing proteins have been identified in D. discoideum (leading to the renaming of DdLim in DdLimE) and several of them colocalize with the actin cytoskeleton (Khurana et al. (2002a), Khurana et al. (2002b)), while LimB is included in the chemotactic sensory pathways (Chien et al., 2000). The LimE-GFP label was used in this study to visualize the actin dynamics under periodic stimulation. Prior (and next) to this study it was successfully applied to visualize the above introduced actin waves (Bretschneider et al. (2004), Bretschneider et al. (2009)). Of particular interest is the study by Diez et al. (2005), which applied TIRF-imaging of LimE-GFP to investigate the subsecond actin filament displacement.

\subsection{The Dictyostelium discoideum chemotactic signal processing system}

Chemotaxis is defined to be the directed movement towards (positive chemotaxis) or away (negative chemotaxis) from a chemical stimulus and plays a crucial role within many eukaryotic processes, such as the bacteria hunting of neutrophils (Servant et al., 2000), the growth of neurons (Rubel and Cramer (2002), Crone and Lee (2002)), the inflammation responses (Baggiolini, 1998) and cancer metastasis (Condeelis et al., 2005). The chemotaxis of mammalian neutrophils and $D$. discoideum are the most intensively studied chemotactic systems (Swaney et al., 2010) and interestingly they share many similarities in terms of the response to extracellular cues and shallow gradients (Bagorda and Parent, 2008). Generally, one can subdivide the process of chemotaxis into three main processes (see Devreotes and Janetopoulos (2003), Franca-Koh and Devreotes (2004), Rappel and Loomis (2009)):

1. Directional sensing, the recognition of an external stimulus and its gradient.

2. Polarization of the cell with a stable leading edge.

3. Directed migration, the final movement of the cell towards the stimulus.

${ }^{6}$ The Phalloidin staining often serves as a control of the suitability of the respective new filamentous actin label. 
A simplified sketch of these processes in the framework of chemotaxis is given in figure 1.3 below (see also Swaney et al. (2010) for a current review).

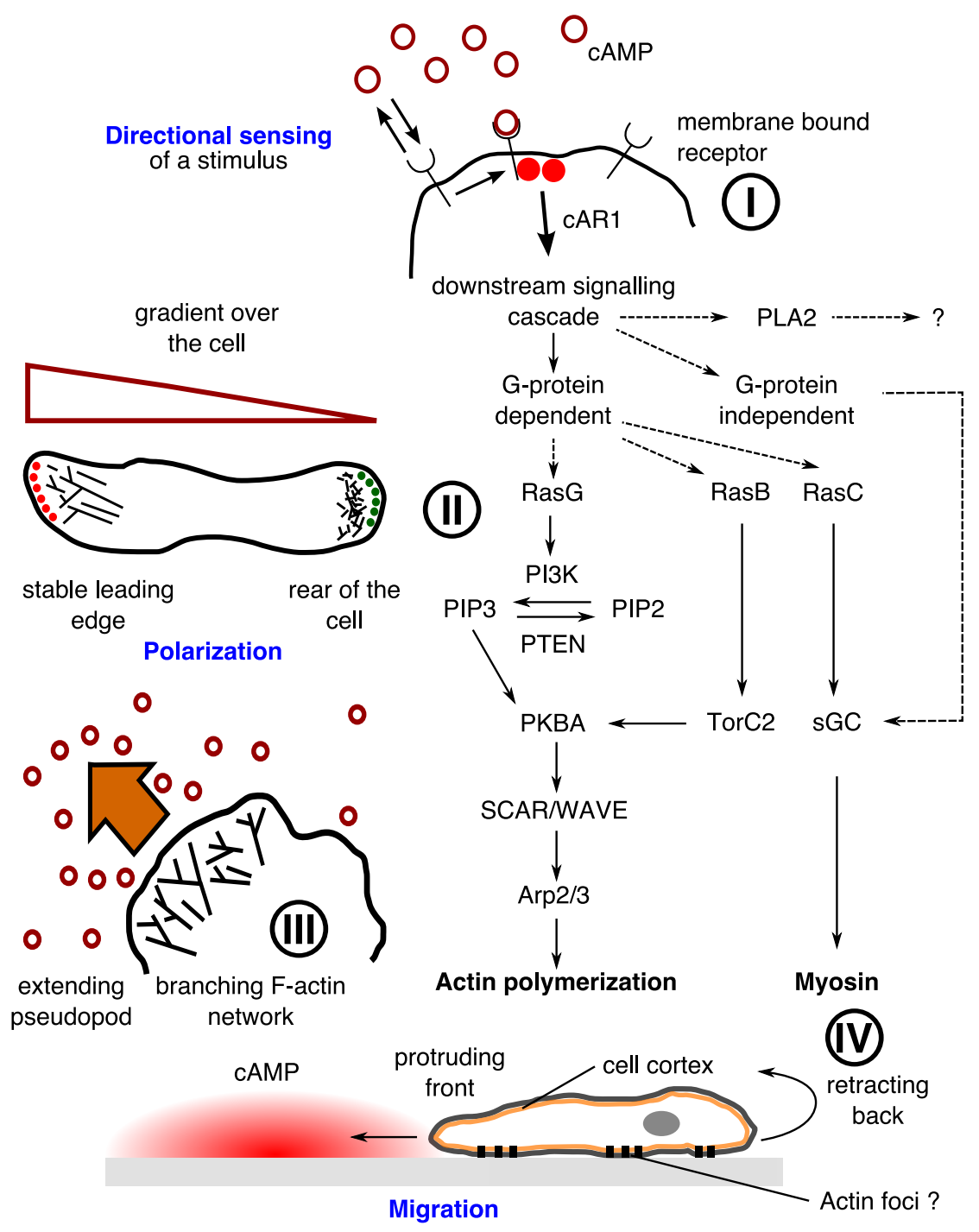

Figure 1.3.: Simplified sketch of the chemotaxis of D. discoideum. In principle the cell senses the presence of the chemoattractant via membrane-bound receptors. This is translated into an internal gradient by a downstream signalling cascade. The cell becomes polarized with a stable leading edge at the front. Finally, D. discoideum migrates towards the source of cAMP. While the front is protruded by the action of the branching and polymerizing actin network, the rear of the cell is retracted by the contracting acto-myosin network. Cell-substrate interaction is possibly mediated by actin foci. Further details are given in the text. 
In the case of developed $D$. discoideum, cAMP acts as a first messenger and binds to the cAMP receptor (cAR1). The cAMP receptor is a transmembrane protein and G-protein coupled receptor (often abbreviated $\mathrm{GpCr}$ ), via which it activates the downstream signalling cascades (see figure 1.3 (I)). For the sake of completeness we note that 4 different cAR receptors are found in the genome of $D$. discoideum (Eichinger et al., 2005). cAR1 and cAR3 are both important in the chemotaxis, with cAR1 beeing the first to be expressed and the more abundant. In the later multicellular stages cAR2 and 4 become important (Parent and Devreotes, 1996).

The G-protein dissociates upon binding of cAMP to the receptor and activates different GTPases via the action of GDP exchange factors. Particularly important is the small GTPase RasG, which activates the Phospho-inositol-3-kinase (PI3K). This enzyme realizes the phosphorylation of the membrane bound phospholipid PIP2 to PIP3 which acts as an anchor for several downstream effector proteins. The opposite reaction is mediated by the phosphatase PTEN, which accumulates at the rear of the cell. This is important, since it is assumed that the external gradient is translated into an internal gradient. The PI3K and PTEN now counteract each other and lead to different biochemistry in the front and in the back, i.e. different downstream effectors binding to the membrane at the front than at the back. Thus the cell is now polarized, with respect to the external gradient (figure 1.3 (II)). The biological picture of the chemotactic response is still incomplete, since different possibly redundant signalling mechanisms were discovered. For example, Veltman and van Haastert (2008) showed that at least 4 different signalling pathways exists. Little is known about the action of the Phospholipase A2 (PLA2), but its importance within the chemotactic response has been proven (Chen et al., 2007). The so-called cGMP pathway relies on the soluble Guanylyl-cyclase (sGC) and is thought to control the response of the motor protein myosin (Veltman et al., 2008). Furthermore, it remains unclear whether there are responses mediated without the dissociation of the G-protein. Interactions between proteins, which have not been fully understood yet, are displayed by dashed arrows in figure 1.3.

The final increase of actin polymerization close to the place of stimulus reception is linked to the nucleation promoting factors. We have seen previously that the action of the Arp2/3complex increases the number of free barbed ends by filament branching and is coupled to the above explained signalling cascade by the action of the SCAR-complex. However, the chemotactic response is not a one way signal processing. Positive feedback loops are established between the F-actin and the Ras-GTPase (Sasaki et al. (2004), Sasaki et al. (2007)). This self-enhancing process finally leads to the actin-driven protrusion of pseudopods (figure 1.3 (III)). It is debated, whether an internal gradient is built up as a representation of the external gradient (as described above), or if a bias on the pseudopod formation leads to 
the directed movement. In the so-called pseudopod-centered approach to chemotaxis, the chemoattractant places a bias on the pseudopod formation and its lifetime (i.e. pseudopods extended away from the chemoattractant source have on average a shorter lifetime and are retracted faster) (Andrew and Insall (2007), Van Haastert (2010)). To contract the rear end of an amoeboid moving cell, the actin network, i.e. the crosslinked network of actin fibers, pulls the rear membrane forward by contraction of the network. This process is driven by the myosin motors (Iwadate and Yumura, 2008). These proteins have a conserved N-terminal motor domain, which drives the movement and variable side chains (de la Roche and Cote, 2001), which are target of different effector proteins. Next to the actin network contraction, the myosin motors serve as transporters moving attached vesicles along the actin filament (figure 1.2G). The contraction of the actin-myosin network requires the network to be attached to the substrate, in order to pull the membrane. Among animal cells this is achieved via the so-called Integrins: heteromeric transmembrane proteins acting as an anchor. These integrins have not been discovered in D. discoideum and it is debated whether the actin foci act as an replacement. Actin foci are spots of high F-actin concentration (Uchida and Yumura, 2004) and are localized on membranes, which are attached to the surface (Skoge et al., 2010). The spatially and temporally coordinated action of actin-driven pseudopod extension and actin-myosin mediated retraction of the cell back finally leads to the migration of the cell towards the chemoattractant (figure 1.3 (IV)).

We should note that the engaged processes within the chemotactic response are transient processes. Most, but not all, responding proteins adapt to the level of external chemoattractant. In fact, one distinguishes adapting and non-adapting proteins. The G-protein stays dissociated as long as the cell receives an external stimulus, while most other key players adapt with their respective timescales (Swaney et al., 2010). The unbinding constant of the cAR1 receptor is between 0.2 and $1.1 \mathrm{~s}^{-1}$ (Janssens and VanHaastert (1987), Ueda et al. (2001)), which means that practically immediately after the cAMP stimulus is washed away the receptors are free again. Examples of rapidly activated proteins are the sGC (peak of activity $1 \mathrm{~s}$ after stimulus (Van Haastert, 1987)) and the RasGTPase (ca. 3 s (Sasaki et al., 2004)). The actin cytoskeleton as well as LimE-GFP respond as early as $3 \mathrm{~s}$ after the stimulus, but reach their maximum activity after 5 - 10 s (Hall et al. (1988) and own observations). This sets the timescale for the directional response to $0-10 \mathrm{~s}$ after the reception of the pulse. The front/back asymmetry between PI3K and PTEN requires $10-15 \mathrm{~s}$ to be build up and finally, after 20 - 30 s the Myosin localization reaches its maximum (Etzrodt et al., 2006). Although the three chemotaxis subprocesses are overlapping each other, the polarization of the cell is believed to take place between $10-60 \mathrm{~s}$ after the stimulus reception, with the net migration setting in afterwards (Rappel and Loomis, 2009). 


\subsection{Probing the actin cytoskeleton}

As cAMP engages the chemotactic response in $D$. discoideum and consequently leads to a reorganization of the actin cytoskeleton, we used this effect to study the actin cytoskeleton in vivo by applying external stimuli of cAMP. Within this work, cAMP was released by a controlled photochemical lysis reaction from a biologically inactive so called caged precursor molecule.

\subsubsection{Caged compounds}

Photoactivatable Nitrobenzyl compounds were first introduced by Barltrop et al. (1966). More then 10 years later, this protecting group had been used to chemically convert cAMP to a biologically inert but photosensitive compound (Engels and Schlaeger, 1977). The term 'caged compound' was introduced in 1978 by Kaplan et al. (1978), who synthesized the first caged ATP molecule. The main advantage of the application of caged compounds is the fast and spatially-restricted release of biological effector molecules. As light source served either a UV-flash lamp or a low-wavelength laser (McCray et al., 1980). The laser based photolysis was applied in this thesis. Uncaging efficiency and kinetics were subsequently improved, by changing the chemical nature of the caging group. The 4,5-dimethoxy-2nitrobenzyl (DMNB) cage, used in this work, was first introduced by Nerbonne et al. (1984) and was widely used over the following decades. A brief overview of the DMNB-caged compound chemistry is given in figure 1.4. The synthesis of caged compounds yields axial and equatorial diastereomers. DMNB-caged cAMP diastereomers have only subtle differences concerning their absorption maxima and quantum yield. However, the equatorial isomer is much more stable in solution than its axial counterpart. The photolysis reaction has an uncaging rate of $k_{1}=300 \mathrm{~s}^{-1}$, i.e. the uncaging of one molecule requires $3 \mathrm{~ms}$ (Hagen and Kaupp, 2005). The photolysis products are the free cAMP, the 2-dimethylnitrosobenzaldehyde and a proton. In aqueous solution the ester bond of the caged cAMP may also spontaneously hydrolyze, releasing the cAMP and the respective alcohol.

The uncaging technique is typically applied, whenever spatially and temporally well defined and restricted stimuli are necessary. A classic example is the activation of cyclic nucleotidegated channels by uncaging DMNB-caged cGMP (the guanosin analog to cAMP) (Karpen et al. (1988), Hagen et al. (1996)) and more recently caged cGMP derivates have been also applied to investigate sperm chemotaxis (Struenker et al. (2011), Boenigk et al. (2009)). 
In the Dictyostelium discoideum research community caged cAMP was used to examine $D$. discoideum chemotaxis, by uncaging a point source close to a single D. discoideum cell (Samadani et al. (2006), Amselem (2010)). By diffusion, this creates a gradient in order to study the polar response of certain signalling cascade effector proteins.

Neither cAMP nor the photolysis product are fluorescent and therefore one cannot quantify the amount of released cAMP directly. DMNB-fluorescein (only the released fluorescein is actually fluorescent) was used to quantify the uncaging efficiency (see for instance Saucerman et al. (2006)), since the caging group is the same. However, comparability remains an assumption. The leaving group kinetics of the fluorescein and the cAMP are similar but not the same. The solvability of DMNB-caged compounds is limited and requires the primary solution to be in Dimethyl sulfoxide (DMSO). The stock solution is diluted by a factor of 1:1000, leaving only small and non-toxic amounts of DMSO in the final solution applied within the experiments.

A

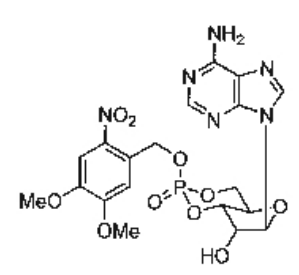

B<smiles>COc1cc(CO)c([N+](=O)[O-])cc1OC</smiles>

C<smiles>COc1cc([N+](=O)[O-])c(OC)cc1C=O</smiles>

D

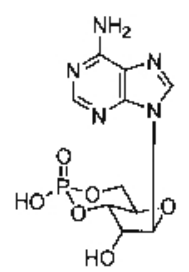

Figure 1.4.: Chemistry of DMNB caged compounds. (A) DMNB-caged cAMP. (B) Product of the hydrolysis of DMNB-caged cAMP. (C) Product of the photolysis of DMNB-caged cAMP. (D) Free cAMP.

\subsubsection{Flow photolysis}

The combination of the light induced release from a caged precursor and microfluidic flow has been developed recently and termed flow photolysis (Beta et al., 2007). The principle of this technique is shown in figure 1.5. The caged compound is flown through a straight microfluidic channel (figure $1.5 \mathrm{~A}$ ) at a certain velocity. A low-wavelength confocal laser is used to scan a certain region (figure $1.5 \mathrm{~B}$, marked in red with the magnification) within the flow. Scanning photolytically releases the respective compound and the compound is immediately dragged away by the microfluidic flow. The result was visualized by uncaging fluorescein from DMNB-caged fluorescein and is shown in figure 1.5C. Advantages of the flow photolysis technique are the very fast concentration switching time and the high degree 
of spatial control (Beta, 2009). The example given in figure $1.5 \mathrm{C}$ has a straight line as the uncaging region, but in principle arbitrary shaped regions can be created and bleached by the laser. In the experiments, carried out within this work, a $405 \mathrm{~nm}$ laser was used to release the caged compound and a second laser was used to acquire the actual confocal image. We shall from now on designate the low-wavelength laser as the uncaging laser and its scanning region as the uncaging region. The flow photolysis method allowed us to create a variety of different temporal input functions by uncaging of caged cAMP upstream of a single $D$. discoideum cell. The temporal resolution is limited by Taylor dispersion (Taylor, 1953). We can understand this phenomenon by thinking of the evolution of an infinitesimally small stripe of some dye in a cylindrical pipe flow. The stripe gets deformed by the parabolic flow profile, but at the same time radial diffusion smoothes the concentration profile. The diffusion therefore counteracts the axial spreading and results in a plug moving with the average flow speed. Taylor dispersion ultimately destroys spatial patterns, created by the flow photolysis. Hence the cell, which is to be stimulated, must be located very close to the uncaging region (about $20 \mu \mathrm{m}$ in the later experiments) to limit the effects of Taylor dispersion.

A

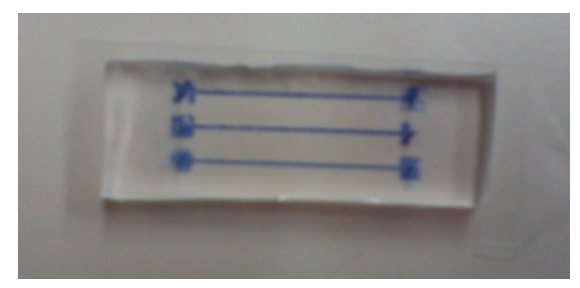

B

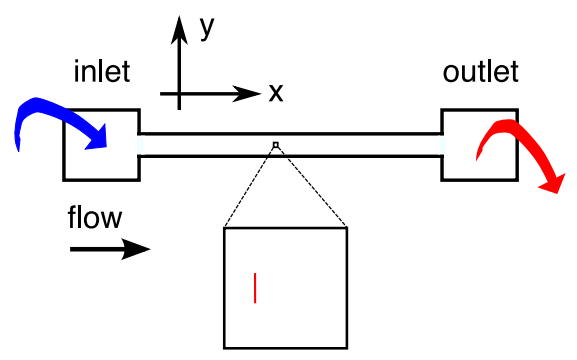

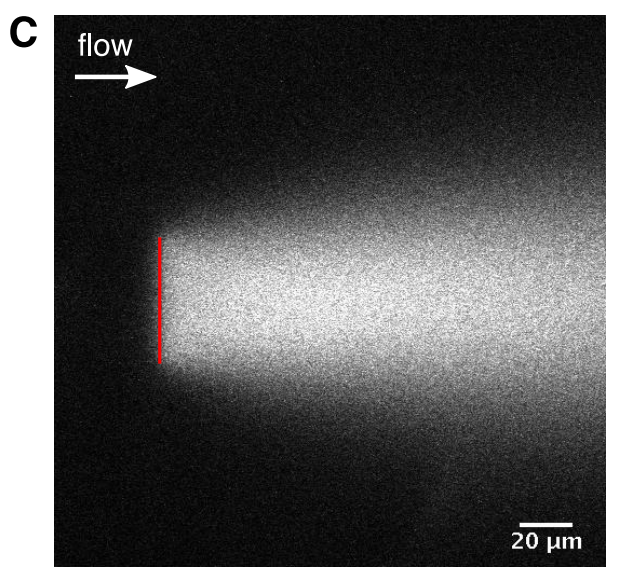

Figure 1.5.: Principle of flow photolysis. (A) Straight microfluidic channel filled with ink. A sketch of the microfluidic channel is given in (B). Caged compounds are supplied via the inlet. The magnification shows the imaging region (black) and the uncaging region (red) used in (C). DMNB-caged fluorescein is uncaged in the microfluidic flow and (C) shows the steady state profile following constant uncaging (uncaging region marked in red). 


\subsection{Linear and non-linear systems}

In the most straightforward case the properties of a system (S) can be described by the output or response $(y(t))$ of the system to a certain well-defined input $(x(t))$ (sketched in figure 1.6A). If the system is linear and the system's impulse response $(h(t))$, i.e. the system's response to a $\delta$ input, is known, then every output can be predicted by the convolution of the impulse response with the respective input. This is known as linear response theory, which originates from statistical mechanics (Kubo, 1957) and was widely applied in other fields, especially electronics. To probe the system's properties, the system's detailed structure is not necessarily known. In fact, the system is treated as a black box with some signal processing properties.

\subsubsection{Linear time invariant systems}

Considering the process of chemotaxis, linear response theory was successfully used to model and to further characterize the chemotactic response of E. coli. This response can be described as the bias of the flagella motor protein rotation (clockwise versus counterclockwise) from its equilibrium ratio (without chemoattractant in the surrounding medium). Linear response theory became applicable to $E$. coli chemotaxis by satisfying the following three conditions:

- Causality - If the input $x(t)=0$ then also the response remains zero. E.coli motion is well known to be indistinguishable from a random walk, if no chemoattractant is apparent in the surrounding medium (Berg, 2003).

- Linearity - If the E. coli bacterium is adapted to the current local extracellular chemoattractant concentration and the following input does not reach the saturation level of the receptors, then the response is linearly related to the input (Segall et al., 1986).

- Stationary - The system can be assumed to be stationary over the course of an experiment.

Furthermore, using a course-grained model approach (Tu et al. (2008), Shimizu et al. (2010), Celani and Vergassola (2010)), the systems pulse response can be derived analytically and was verified experimentally. An analytical solution does not suffer from the limitations of the experimental delta function realization. The description of the E. coli chemotaxis as a linear time-invariant system has been very successful. However, recent discoveries suggest non-linearities in its chemotactic response (Min et al., 2012). 


\subsubsection{Superposition principle}

This section summarizes the argumentation in Marmarelis (1978). Let $y_{a}(t)$ be the systems response to $x_{a}(t)$ and $y_{b}(t)$ the response to $x_{b}(t)$. If the system is linear, the superposition of both responses equals the actual measured response (see figure 1.6B). Including an amplitude $\left(a_{n}\right)$, every input can be described as a sum of known input functions:

$$
a_{1} x_{1}(t)+a_{2} x_{2}(t)+\ldots+a_{n} x_{n}(t)
$$

and produces the output

$$
a_{1} y_{1}(t)+a_{2} y_{2}(t)+\ldots+a_{n} y_{n}(t) .
$$

This is called the superposition principle. However a practical realization (especially in biological experiments) of a $\delta$ input function is complicated. Examples from neurobiology show the application of light pulses to the neurons, which is probably the most accurate representation of a $\delta$ input (Sakai and Naka (1987)). These timescales cannot be reached if the applied stimulus is a chemical in solution. However, as has been shown previously (Bae et al., 2009), the above introduced flow photolysis method provides the fastest switching mechanism of a chemical in a microfluidic flow.

If the stimulus has necessarily a finite time dimension, then the superposition can be written as the sum of rectangular pulses of time extension $\Delta t$, and consequently the response is the sum of the individual responses to the rectangular pulse:

$$
y(t)=y(N \Delta t)=\sum_{k=0}^{N} \Delta t x(k \Delta t) h(t-k \Delta t)
$$

with $h(t-k \Delta t)$ being the approximated impulse response (to a rectangular shaped pulse with finite time extension $\Delta t$ ). Only in the limit of $\lim _{\Delta t \rightarrow 0}$ and with $k \Delta t=\tau$, the sum becomes the integral:

$$
y(t)=\int_{0}^{t} x(\tau) h(t-\tau) d \tau
$$

or respectively,

$$
y(t)=\int_{0}^{t} x(t-\tau) h(\tau) d \tau
$$

with $x(t)=0$ for $t<0$ (causality). This is the convolution integral of the impulse response $h(t)$ with an arbitrary shaped input $x(t)$. The linear response theory supplies us also with a definition of nonlinearities. Easily, if the superposition of responses fails, nonlinear processes must be taken into account. This is sketched in 1.6B. The dashed response $\left(y_{c}(t)\right)$ is not the superposition of the two single responses $\left(y_{a}(t)+y_{b}(t)\right.$, solid line). 
A system

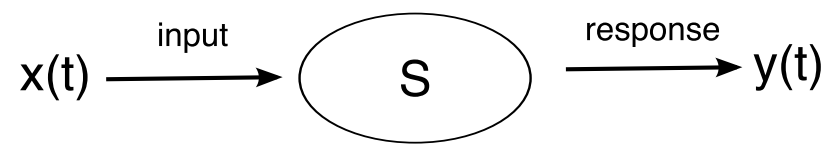

B
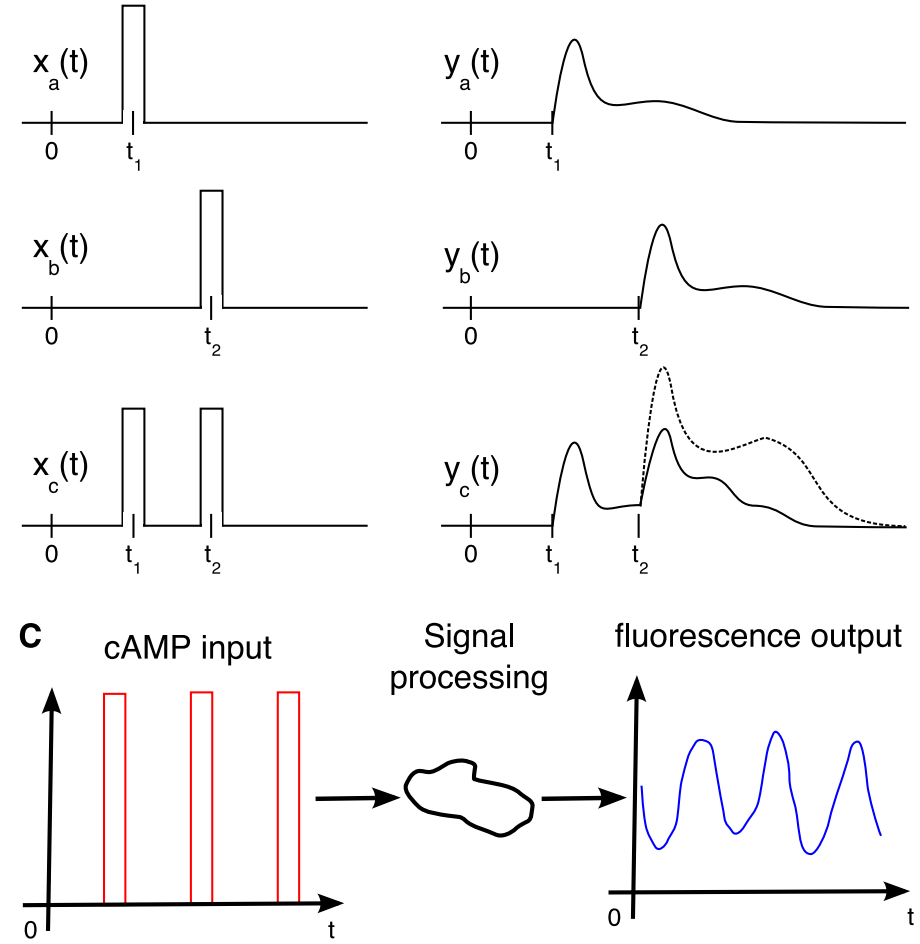

Figure 1.6.: Characterization of a signal processing system. (A) An arbitrary system with signal processing properties (S) can be characterized by the response $(y(t))$ to well defined inputs $(x(t))$. (B) The superposition principle. If the superposition of both individual responses $y_{a}(t)+y_{b}(t)$ is not equal to the observed response $y_{c}(t)$, then the system needs to be considered as non-linear. (B) was adapted from Marmarelis (1978). (C) Sketch of the characterization of the D. discoideum signal processing system and its actin polymerization machinery. The input is realized as cAMP pulses created by flow photolysis. The output is measured as the fluorescence time profile of labelled F-actin associated proteins.

Finally, this leads us to the scope of this thesis, which is sketched in 1.6C. Within this work, the oscillatory dynamics of the eukaryotic actin cytoskeleton in developed and chemotactic D. discoideum amoebae was studied. The cytoskeleton and its regulating signal processing system are treated as a black box, which is acting on the input. The input were almost rectangular pulses of cAMP, created by the flow photolysis method. Finally, the output the signal processing is measured using fluorescent labels of the filamentous actin system. 
CHAPTER 2

Material and methods

\subsection{Cell culture}

Dictyostelium discoideum was stored at $-80^{\circ} \mathrm{C}$ as either cells or spores for an extended period. Cells were frozen in HL-5 medium $(14 \mathrm{~g} / \mathrm{L}$ peptone, $7 \mathrm{~g} / \mathrm{L}$ yeast extract, $13.5 \mathrm{~g} / \mathrm{L}$ glucose, $0.5 \mathrm{~g} / \mathrm{L} \mathrm{KH}_{2} \mathrm{PO}_{4}, 0.5 \mathrm{~g} / \mathrm{L} \mathrm{Na}_{2} \mathrm{HPO}_{4}$, purchased from Formedium, Norwich, England) (Watts and Ashworth, 1970) with additional 20\% Dimethyl sulfoxid (DMSO, Calbiochem, Darmstadt, Germany) and 40\% Fetal calf serum (FCS, Invitrogen), while spores were frozen directly in liquid nitrogen. The culture of $D$. discoideum was maintained in HL5 medium at $22{ }^{\circ} \mathrm{C}$ in a Petri dish (Fey et al., 2007). The cell culture was diluted and its medium refreshed every $2-3$ days, depending on the cell density. The culture itself was renewed from frozen stock once a month. The added amount of the antibiotics Genetecin (G418, Roche, Mannheim, Germany and Gibco, Darmstadt, Germany) and Blasticidin (Sigma Aldrich, Taufkirchen, Germany), to maintain the introduced mutations, varied with the cultured mutant. An overview of the strains, investigated within this work and their requirements is given in table 1.

The preparation of cells for flow photolysis experiments (see section 2.3) started one day prior to the experiment being carried out. An inoculum of $1 \times 10^{6}$ cells was pipetted into $25 \mathrm{ml} \mathrm{HL} 5$ medium and cultivated at $22^{\circ} \mathrm{C}$ as a shaking culture ( $\left.150 \mathrm{rpm}\right) .7$ hours in advance of the experiment the full shaking culture was centrifuged $\left(1000 \mathrm{rpm}, 3 \mathrm{~min}, 4{ }^{\circ} \mathrm{C}\right)$, washed once in phosphate buffer $(\mathrm{PB}, \mathrm{pH}=6)$ and the remaining pellet was diluted in 


\begin{tabular}{ccccc}
\hline Description & $\begin{array}{c}\text { additional } \\
\text { Name }\end{array}$ & Background & $\begin{array}{c}\text { Geneticin } \\
\mu 1 \text { in } 10 \mathrm{ml}\end{array}$ & $\begin{array}{c}\text { Blasticidin } \\
\mu 1 \text { in 10 ml }\end{array}$ \\
\hline LimE-GFP & $4-2-D 6$ & AX2 & - & - \\
LimE-GFP & - & AX3 & 5 & - \\
SCAR(-)/PIR121(-) LimE-GFP & - & AX3 & 10 & - \\
MyoII-GFP LimE-mRFP & - & AX2 & 5 & 10 \\
Aip1-GFP LimE-mRFP & - & AX2 & 5 & 10 \\
Coronin-GFP LimE-mRFP & - & AX2 & 5 & 10 \\
CRAC-GFP & Wf-38 & AX3 & 5 & - \\
Actin-GFP & - & AX2 & 5 & - \\
cytosolic GFP & HG1694 & AX2 & 5 & - \\
cAR1-GFP & - & AX2 & 5 & - \\
\hline
\end{tabular}

Table 2.1.: List of all used Dictyostelium discoideum mutants, their axenic background strain and the antibiotics added to each cell culture. Literature references for each strain are given in the text of the respective results sections. Two strains with different axenic backgrounds for LimE-GFP exist. If not mentioned otherwise the 4-2-D6 strain was used.

$20 \mathrm{ml} \mathrm{PB}$. This new shaking culture $\left(150 \mathrm{rpm}, 22^{\circ} \mathrm{C}\right)$ was subject to periodic pulses of $3^{\prime}-5^{\prime}$ cyclic adenosine monophosphate (cAMP, Sigma-Aldrich) in order to enhance the development of the D. discoideum amoebae (Chisholm et al., 1987). A pulse of cAMP consisted of approximately $60 \mu \mathrm{l}$ with a concentration of $18 \mu \mathrm{M}^{1}$ and was added every 6 minutes. The cells developed for 5 hours. 1 hour prior to the experiment the cells were washed $1-3$ times in PB (1000 rpm, $3 \mathrm{~min}, 4^{\circ} \mathrm{C}$ ) and were finally dispersed in $1-2 \mathrm{ml} \mathrm{PB}$ (depending on the cell density). This cell solution was then ready to be placed into a microfluidic channel.

\subsection{Microfluidics}

Soft lithography (for reviews see Xia and Whitesides (1998), Whitesides et al. (2001) and Qin et al. (2010)) was used to create microfluidic channels. This process can be subdivided into three different processes (see figure 2.1): (A) the creation of a chrome photomask, (B) the generation of a reusable master wafer and finally (C) the replica molding of the microfluidic channel. The generation of a photomask first required the desired geometry to be drawn in a computer-aided design (CAD) file. The geometries used here are presented in figure 2.2. The geometry was printed onto a photoresist covering the chrome/quartz photomask. This printing required an optical pattern generator (GCA PG3600F). The mask

\footnotetext{
${ }^{1}$ Adding $60 \mu \mathrm{l}$ of $18 \mu \mathrm{M}$ cAMP to the culture results in an overall concentration of $50 \mathrm{nM}$.
} 
was placed in a mask processor (Steag Hamatech, Austin, TX) and exposed to UV light rendering it insoluble. Photoresist that had not been exposed to UV light remained soluble and was subsequently washed away using a photoresist developer. Regions of the chrome no longer coated with photoresist were removed using chromatic acid and the remaining photoresist was then stripped off. The reusable master wafer was generated as a pattern of the photoresist SU-8 on top of a silicon wafer. The polished silicon master wafer (diameter $100 \mathrm{~mm}$, SiMat) was placed onto a $200^{\circ} \mathrm{C}$ heating plate before spin coating, in order to evaporate possible organic solvent residues. After placing the wafer on the spin coater (M-Spinn200, Ramgraber, Hofolding, Germany) a sufficiently large drop of degassed SU825 (Micro Resist Technology, Berlin, Germany) was poured onto the middle of the silicon wafer. The necessary angular velocities and times to create different heights of SU-8 25 can be found in the Microresist references ${ }^{2}$. With the spin coated SU- 8 on top, the wafer was first baked for 10 minutes at $65^{\circ} \mathrm{C}$ and then for 20 minutes at $95^{\circ} \mathrm{C}$. Exposing the baked SU-8 to UV light engages crosslinking reactions. Placed below the chrome photomask the geometry is written into the SU-8. All necessary steps were carried out using the EV620 mask alignment system (EV Group, St. Florian am Inn, Austria). The exposed wafer was baked again (slow temperature ramp from $65^{\circ} \mathrm{C}$ to $95^{\circ} \mathrm{C}$ and back) and developed in photoresist developer (Microresist, Berlin, Germany). Finally, the wafer was cleaned with Isopropanol and the height of the SU-8 pattern was verified using white light interferometry (Wyko NT 1100, Veeco, Plainview, NY).

To create microfluidic channels, the wafer was placed into a sufficiently large weightboat with a well mixed solution of polydimethylsiloxane (PDMS) and curing agent (both Sylgard 184, Dow Corning Europe SA, Houdeng-Goegnies, Belgium). The mixture (100:10) was degassed in a vacuum desiccator and cured for at least one hour at $75^{\circ} \mathrm{C}$. A single microfluidic device (each device consisted of 3 independent channels, see figure 2.2A) was cut out using a sharp knife and removed from the wafer. A clean 19 gauge stainless steel syringe tip (McMaster, Hamilton, Ontario) was used to punch inlets and outlets into the PDMS. In the final step, a cover glass (No.1, 24×60 mm, Menzel Gläser, Braunschweig, Germany) was bonded to the lower surface of the PDMS channel, after 3 - 5 minutes plasma treatment (PDC 002, Harrick Plasma, Ithaca, NY), to seal the microchannels from below. The microfluidic device was incubated at $75^{\circ} \mathrm{C}$ for 5 minutes and then filled with the cell suspension (section 2.1) through PTFE tubing (Novodirect, Kehl, Germany) via a 23 gauge sterile and sharpened syringe (Kruuse, Langeskov, Denmark). Due to the constant change of hydrophobility of the PDMS, the microfluidic device was filled at most 30 minutes after plasma cleaning. Each microfluidic device was only used once.

\footnotetext{
${ }^{2}$ http://www.microresist.de/products/mec/mccprod_en.htm
} 


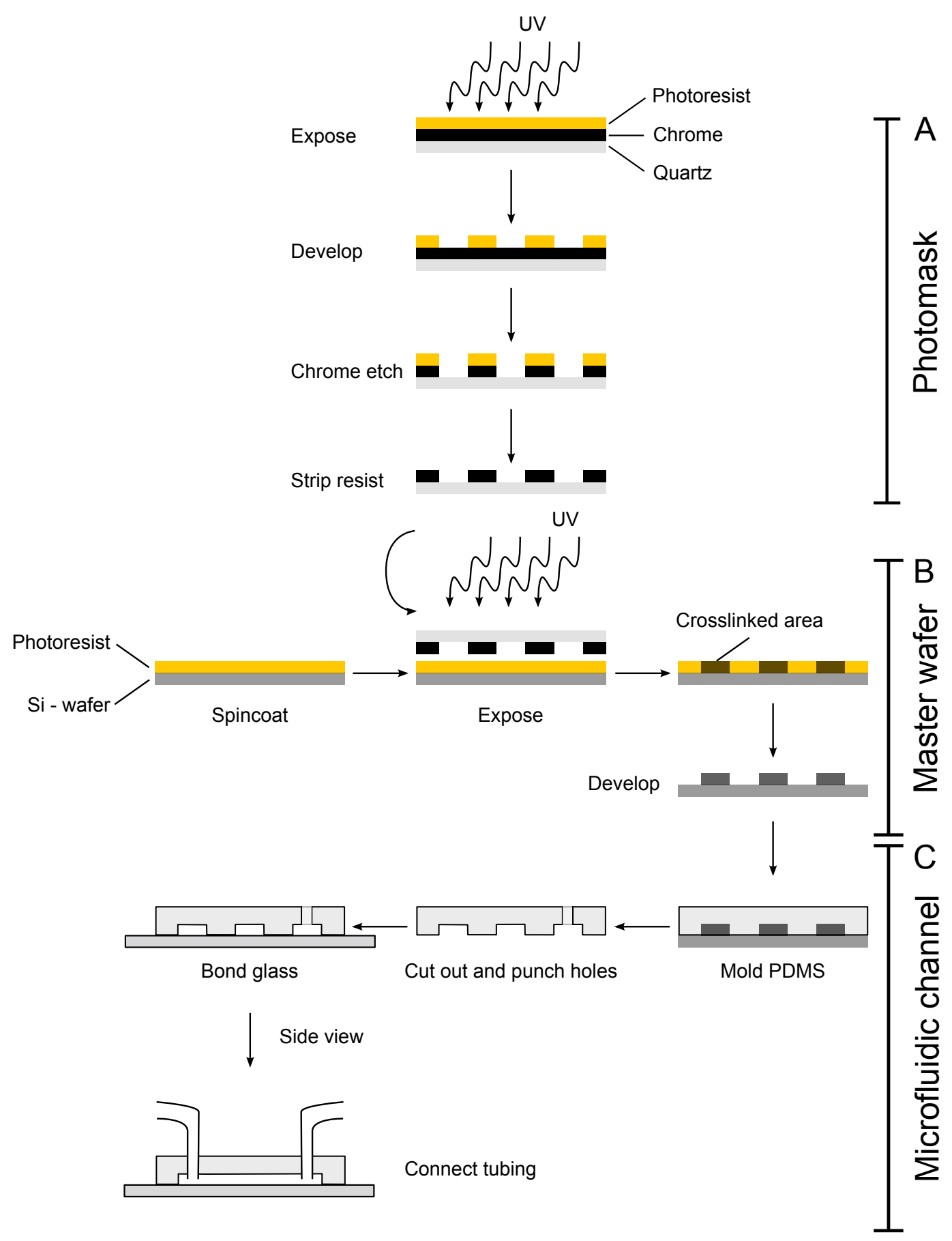

Figure 2.1.: Soft lithography and replica molding. The fabrication of microfluidic channels can be subdivided in three major processes: (A) the creation of the photomask, (B) the creation of the master wafer and finally $(C)$ the molding of a microfluidic channel. Information on the procedures are given in the text. The initial creation of the photomasks was carried out at the Cornell nanofabrication facility and the subsequent steps took place at the cleanroom facilities of the Max Planck Institute for Dynamics and Self-Organization in Göttingen. Figure adapted from Theves (2009) and Bae (2010). 
A

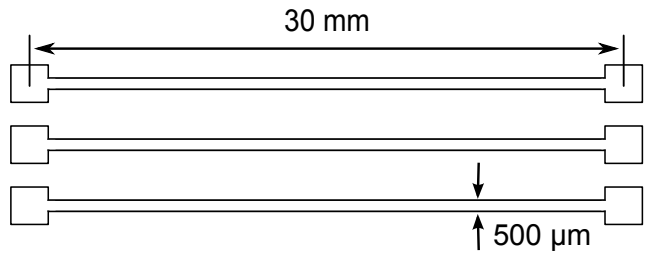

B

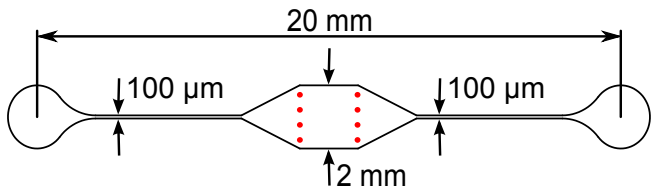

Figure 2.2.: Used channel geometries. (A) The mask containing three straight microfluidic channels for each microfluidic device was used for all periodic stimulation experiments and to create the lower chamber of the double layer flattening devices. (B) shows the high aspect ratio chamber, which was used to create different types of flattening chambers. Red dots indicate later columns of PDMS to prevent the final channel from collapsing (figure taken from Westendorf et al. (2010)). Design of both masks by Danica Wyatt and Eberhard Bodenschatz. The chrome photomasks were created at the Cornell nanofabrication facility by Gabriel Amselem (A) and Danica Wyatt (B).

\subsection{Experimental setup and its characterization}

\subsubsection{Fluorescence intensity characterization}

Confocal laser scanning microscopy was conducted on two Olympus FV 1000 setups. One setup was used for all experiments considering the external driving of cells, while the second setup was applied to investigate the self-sustained actin oscillations ${ }^{3}$. Uncaging of caged cAMP and caged fluorescein respectively required the $405 \mathrm{~nm}$ line of a $35 \mathrm{~mW}$ laser diode (FV5-LD405). GFP fluorescence was excited with the $488 \mathrm{~nm}$ line of a $150 \mathrm{~mW}$ Argon laser (Melles Griot, Carlsbad, CA). Both microscopes were characterized in a similar manner. For all external driving experiments the laser intensity was kept constant, while for the investigation of the self-sustained oscillations the laser intensity was changed as part of the experiment. Therefore we will focus on the characterization of the second setup. $100 \mu \mathrm{M}$ fluorescein solution (Acros organics, Nidderau, Germany) was dropped onto a glass bottom Petri dish (No. 1.5, diameter $50 \mathrm{~mm}$, MaTek, Ashland, MA) and imaged through a 60x UplansApo oil objective. Both the pinhole size $(100 \mu \mathrm{m})$ and the pixel aspect ratio $(512 \times 512)$ were kept constant. The fluorescence intensity was excited with different intensities (10 and $40 \%$ of maximal output) of the $488 \mathrm{~nm}$ laser and recorded with different photomultiplier anode voltages. The calibrated curves of the setup are given in figure 2.3.

\footnotetext{
${ }^{3}$ The second setup had two additional laser light wavelengths at $559 \mathrm{~nm}(20 \mathrm{~mW})$ and $635 \mathrm{~nm}(50 \mathrm{~mW})$.
} 

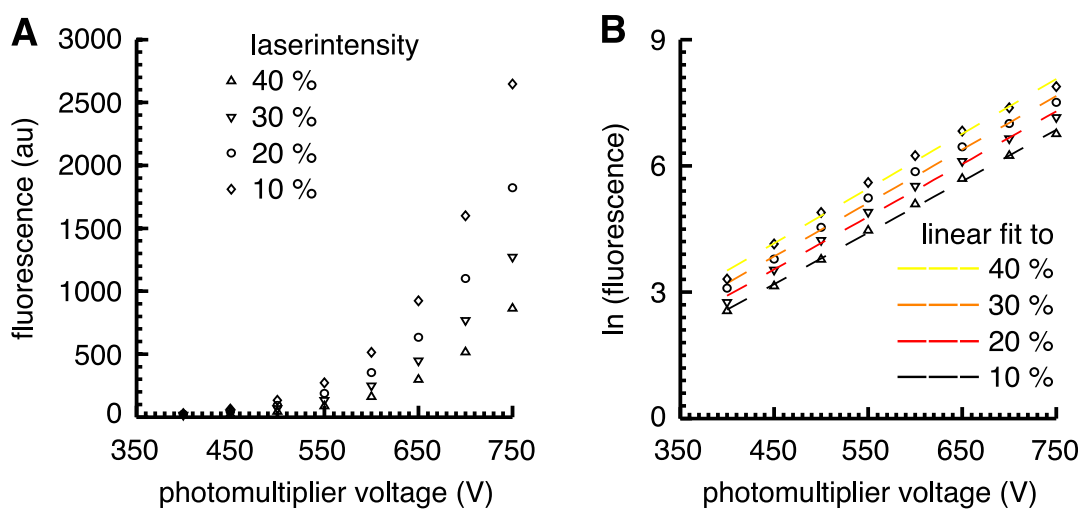

Figure 2.3.: Characterization of the FV1000 confocal laser scanning system. A $100 \mu \mathrm{M}$ fluorescein solution was excited with the $488 \mathrm{~nm}$ line of a $150 \mathrm{~mW}$ Argon laser with different output intensities. The chosen intensities were the same as applied in the later experiments. The emitted fluorescence was recorded using different photomultiplier anode voltages. (A) gives the fluorescence intensities after the background was subtracted. (B) A linear fit was applied to the natural log of the recorded fluorescence intensities. This characterizes the difference of the used laser intensities.

\subsubsection{Mean flow velocity characterization}

The velocity measurement was carried out by uncaging of DMNB-caged fluorescein (MW 3000 , Invitrogen) in the microfluidic flow. This characterized the mean flow velocity in the microfluidic channel approximately $1-2 \mu \mathrm{m}$ above the cover glass surface. The volume flow was $5 \mu \mathrm{l} / \mathrm{h}$ throughout the experiment. The uncaging region was $48 \mu \mathrm{m}$ in length and oriented perpendicular to the flow. The imaging region was chosen to be smaller than in the later experiments, in order to obtain faster acquisition rates (figure 2.4). The plug of fluorescein released by a $0.2 \mathrm{~s}$ uncaging event required approximately $0.4 \mathrm{~s}$ to reach the downstream extension of the imaging region (figure 2.4A and B). By lateral diffusion the plug smears out and becomes wider. Additionally the flow profile appears to be tilted by a certain angle with respect to the flow direction. This is artificially introduced by the limited scanning speed of the imaging laser ${ }^{4}$. We used a Gaussian fit to the sum of lines in the flow direction $^{5}$ (see figure $2.4 \mathrm{C}$ for a single experiment example). The difference of the maxima over time gave the velocity of the flow. For a $26 \mu \mathrm{m}$ high and $500 \mu \mathrm{m}$ wide channel a volume flow of $5 \mu \mathrm{l} / \mathrm{h}$ corresponds to a mean flow ${ }^{6}$ speed of $120 \pm 6.5 \mu \mathrm{m} / \mathrm{s}$.

\footnotetext{
${ }^{4}$ In fact one could calculate the mean flow speed from the slope of the tilted profile, but this method is less accurate.

${ }^{5}$ This used the MATLAB function trackgaussian.m originally written by Albert J. Bae.

${ }^{6}$ The plug moves according to Taylors work (Taylor, 1953) with the mean flow not with the maximal velocity (i.e. the velocity in the center of the microfluidic channel).
} 
A

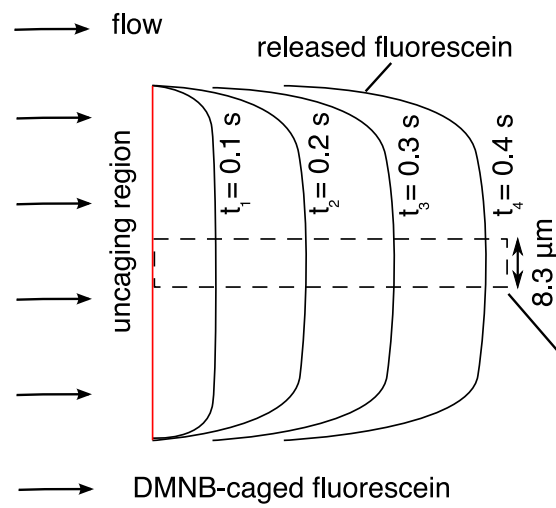

B

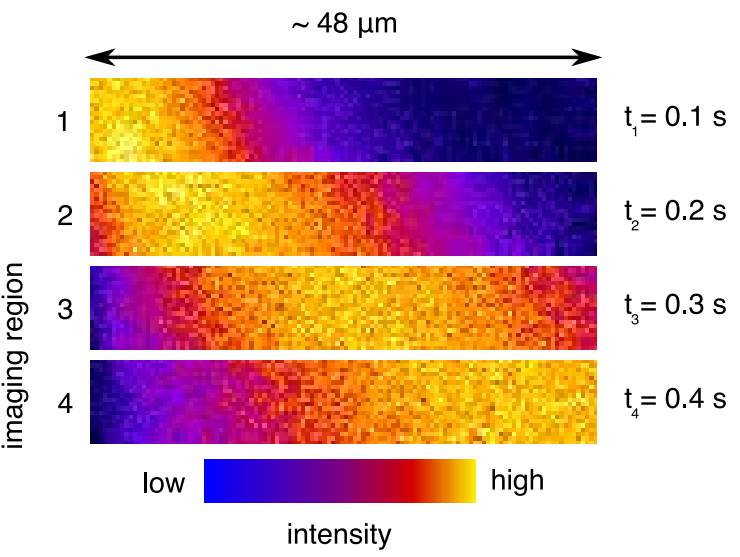

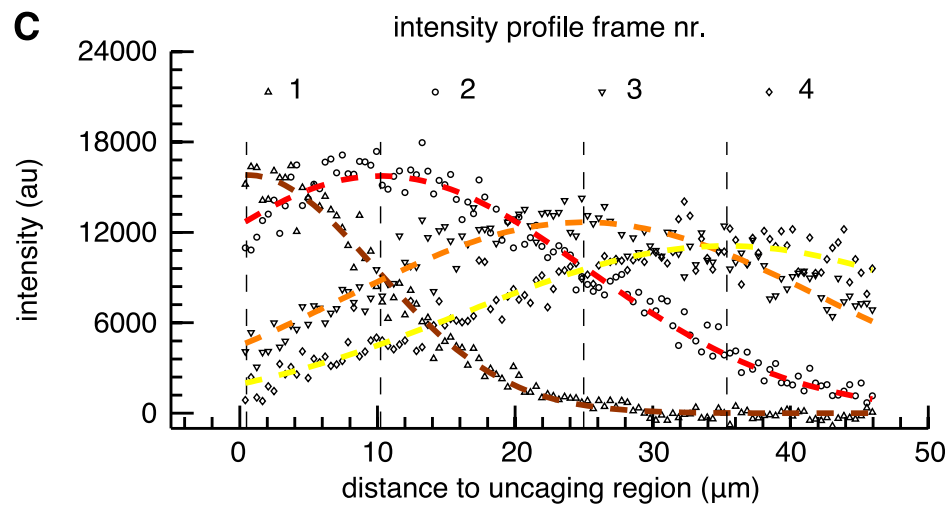

Figure 2.4.: Average flow speed measurement. The microfluidic channel $(500 \mu \mathrm{m}$ width, $26 \mu \mathrm{m}$ height) was connected to a syringe filled with $100 \mu \mathrm{M}$ DMNB-caged fluorescein solution. Characterization was carried out while applying a constant flow rate of $5 \mu \mathrm{l} / \mathrm{h}$. (A) The uncaging region was oriented perpendicular to the flow as used in the caged-cAMP uncaging experiments (see figure 2.6). The scanning region was chosen to be small enough so that scanning times of less than $0.1 \mathrm{~s}$ per frame were obtained. The fluorescein was released by a $0.2 \mathrm{~s}$ lasting uncaging event. It reached the opposite end of the region of interest within $0.4 \mathrm{~s}$ (A, sketched by the black lines). The concentration profile becomes wider due to lateral diffusion. The averaged fluorescence intensity out of 10 independent experiments is given frame by frame in (B). A gaussian was fitted to the sum of all horizontal lines and the difference in the maxima over time gives the velocity. (C) A single representative experiment is shown. The distribution give the summed intensity along the flow direction. Different markers show the summed intensity profiles for the 4 images and the dashed lines give the respective fitted gaussians ${ }^{7}$. 


\subsubsection{Concentration profile characterization}

Since cAMP is non-fluorescent, a similar setup as in the previous section (uncaging of caged fluorescein) was used to evaluate the time profile of the cAMP input function in the external driving experiments (see below). First the fluorescence profile was compared with a finite element simulation (FEM). This was done to justify later use of finite element simulations to estimate the cAMP profile. The uncaging time was extended to $1 \mathrm{~s}$ (as in the actual experiments) and the fluorescence was recorded as in figure 2.4A. Figure $2.5 \mathrm{~A}$ shows the temporal fluorescence profile 20 and $30 \mu \mathrm{m}$ downstream of the uncaging region (black and red dots respectively). The corresponding space time plot is given in figure 2.5B. FEM simulations were carried out using the COMSOL Multiphysics 3.5 software. It solved the convection diffusion equation in two dimensions ( $\mathrm{x}, \mathrm{z}$ ), for $\mathrm{x}=300 \mu \mathrm{m}, \mathrm{y}=200 \mu \mathrm{m}, \mathrm{z}=26 \mu \mathrm{m}$. The uncaging region was simulated as the source region, where the concentration was set to $\mathrm{c}_{0}=1$ for the total time of $1 \mathrm{~s}$ and $\mathrm{c}_{0}=0$ afterwards. Side walls of the simulated microfluidic channel imposed insulation boundary conditions and the concentration at the front of the simulation area was set to 0 . Consequently the back of the simulation area imposed convective flux boundary conditions. Front and back are defined with respect to the flow direction. The diffusion coefficient of uncaged dextrane conjugated fluorescein was set to $\mathrm{D}=267 \mu \mathrm{m}^{2} / \mathrm{s}$ (Bae et al., 2009) and the mean flow velocity was set to $\vec{u}=120 \mu \mathrm{m} / \mathrm{s}$ as shown in the previous section. The concentration profile was evaluated 20 and $30 \mu \mathrm{m}$ downstream (and central in the channel region) from the source. The result is given in figure $2.5 \mathrm{~A}$ by a solid blue line ( $20 \mu \mathrm{m}$ distance) and a solid orange line ( $30 \mu \mathrm{m}$ distance to the uncaging region). Additionally a movie showing the evolution of the cAMP pulse can be found in the electronic appendix. The finite element method provides a good approximation of the fluorescence profile timescales following a $1 \mathrm{~s}$ uncaging experiment ${ }^{8}$. Consequently, FEM simulations were used to estimate the cAMP profile at different distances to the uncaging region with the diffusion coefficient $\mathrm{D}_{C A M P}=440 \mu \mathrm{m}^{2} / \mathrm{s}$ (Dworkin and Keller, 1977). The concentration switching time ${ }^{9}$ is on the order of $0.6 \mathrm{~s}$ and the short lived plateau of nearly constant cAMP concentration is approximately $0.7 \mathrm{~s}$ long. The total duration of the pulse is $1.5 \mathrm{~s}$ (Laser scans for exactly $1 \mathrm{~s}$ ). The timescale dependence of the distance to the uncaging region (here 20 and $30 \mu \mathrm{m}$ downstream) is given in figure $2.5 \mathrm{C}$.

\footnotetext{
${ }^{7}$ Note that the means are not necessarily equally spaced due to the unavoidable jitter in data acquisition.

${ }^{8}$ Note that the amplitude of the data has been scaled to fit the amplitude of the simulation.

${ }^{9}$ The concentration switching time is defined as the time of change from $5 \%$ to $95 \%$ of the final concentration (Bae et al., 2009).
} 

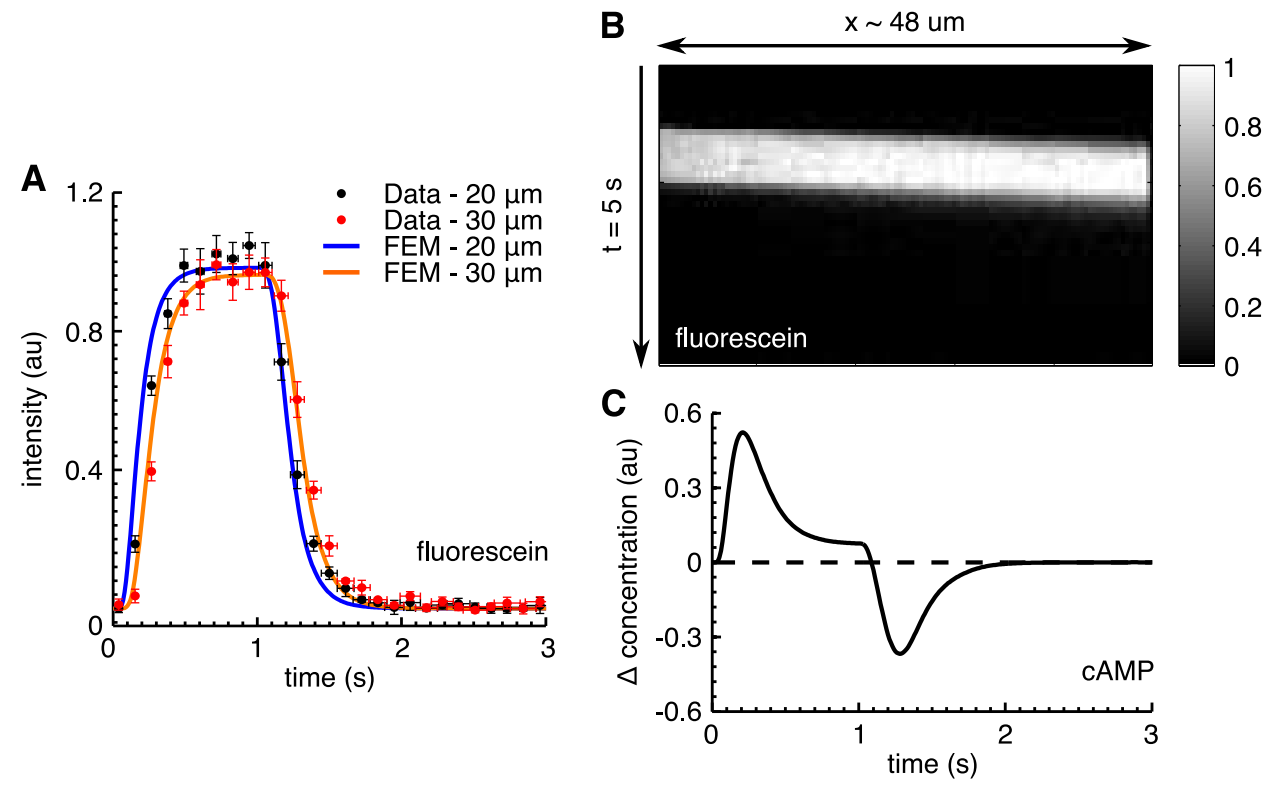

Figure 2.5.: Measured and calculated concentration profiles after uncaging. (A) The temporal fluorescence profile of released dextrane conjugated fluorescein at $20 \mu \mathrm{m}$ (black dots) and $30 \mu \mathrm{m}$ (red dots) downstream of the uncaging region is given with the respective standard deviations in $\mathrm{x}$ and $\mathrm{y}$. Data are the average over 10 independent experiments. The solid lines show the height scaled results of the finite element simulation at the respective distances to the source. (B) shows the averaged space time plot of 10 independent one second lasting uncaging events of DMNB-caged fluorescein. It appears darker at small distances to the uncaging region, due to the slow activation dynamics of the freshly released fluorescein. Changing the diffusion coefficient in the FEM simulation to $D_{\text {cAMP }}$ allows to estimate the concentration profiles for the uncaged cAMP. The difference of the concentrations between 20 and $30 \mu \mathrm{m}$ (approximate position of the front and back of a typical cell) is given in $(\mathrm{C})$.

\subsubsection{Characterization of the DMNB-caged cAMP}

The release of cAMP by photolysis, from its caged precursor, can also occur spontaneously by hydrolysis. Therefore an estimate of the already uncaged amount of cAMP in the solution is necessary. DMNB-caged cAMP was purchased from Invitrogen as dry powder. Prior to dilution with phosphate buffer the powder was solved in DMSO to generate a $5 \mathrm{mM}$ stock solution. Initial characterization of a $200 \mu \mathrm{M}$ DMNB-caged cAMP solution was carried out in the lab of Claudia Höbartner (Max Planck Institute for Biophysical Chemistry, Göttingen, Germany) using high performance liquid chromatography (HPLC). Free cAMP was detected in the polar fraction at a detection wavelength of $260 \mathrm{~nm}$. To investigate whether the entire peak was composed of cAMP required further examination using HPLC in conjunction with Mass spectrometry (MS). A $500 \mu \mathrm{M}$ DMNB-caged cAMP solution was characterized by HPLC-MS in the lab of Ulrich Benjamin Kaupp at CAESAR 
in Bonn, Germany ${ }^{10}$. It revealed that the purchased DMNB-cAMP solution consists of its equatorial and axial isomer. Free cAMP appears in the strongly polar fraction of the HPLC, but MS showed that this fraction is still corrupted with other polar components from the buffer solution. Direct quantification is thus not possible. However, the second hydrolysis product (see figure 1.4C) was estimated at about $1 \%$ of the total mass fraction. It appears in a 1:1 ratio to cAMP, but the absorption coefficient at $254 \mathrm{~nm}$ (HPLC detection wavelength) is not necessarily the same and therefore the value of $1 \%$ background concentration of free cAMP remains an estimate.

\subsubsection{Experimental setup}

The microfluidic channel (filled with the cell suspension, see section 2.1) was connected to a $10 \mu \mathrm{M}$ DMNB-cAMP (Invitrogen) containing syringe and mounted on the stage of the confocal laser scanning microscope. The cells were allowed to settle for 15 minutes and afterwards the PB was replaced with the caged cAMP by engaging a volume flow of $10 \mu \mathrm{l} / \mathrm{h}$ for 30 minutes via the connected syringe pump (PHD2000, Harvard Apparatus). For the duration of the experiment the flow was reduced to $5 \mu \mathrm{l} / \mathrm{h}$. Each independent experiment was conducted as sketched in figure 2.6. A $48 \times 48 \mu \mathrm{m}(120 \times 120$ pixel $)$ squared imaging region was aligned with a line shaped uncaging region located upstream (oriented perpendicular to the flow). The $488 \mathrm{~nm}$ Argon laser scanned the imaging region in parallel mode with a pixel resting time of $2 \mu \mathrm{s}$. The $405 \mathrm{~nm}$ laser scanned the uncaging line at a rate of $0.25 \mathrm{pixel} / \mu \mathrm{s}$. Simultaneous imaging and uncaging was possible due to the second set of galvano mirrors included in the Fluoview 1000 SIMultaneous setup. Prior to each experiment a motile and elongated single cell was centered within the imaging region. The experiment started with recording the fluorescence fluctuations for $20-30 \mathrm{~s}$ as a control and afterwards the different stimuli were applied. Periodic pulses were given for a maximal stimulation time of $320 \mathrm{~s}$. After finishing one experiment the next cell was located by moving the region of interest upstream or perpendicular to the flow (figure 2.6) to ensure unstimulated cells. Since the D. discoideum amoeba develops under starvation conditions the total experimental time for one cell suspension was limited to 3 hours (i.e. the time from 7 to 10 hours after the amoebae were depleted from food).

${ }^{10}$ Experiments carried out by Nico Kotzur. 


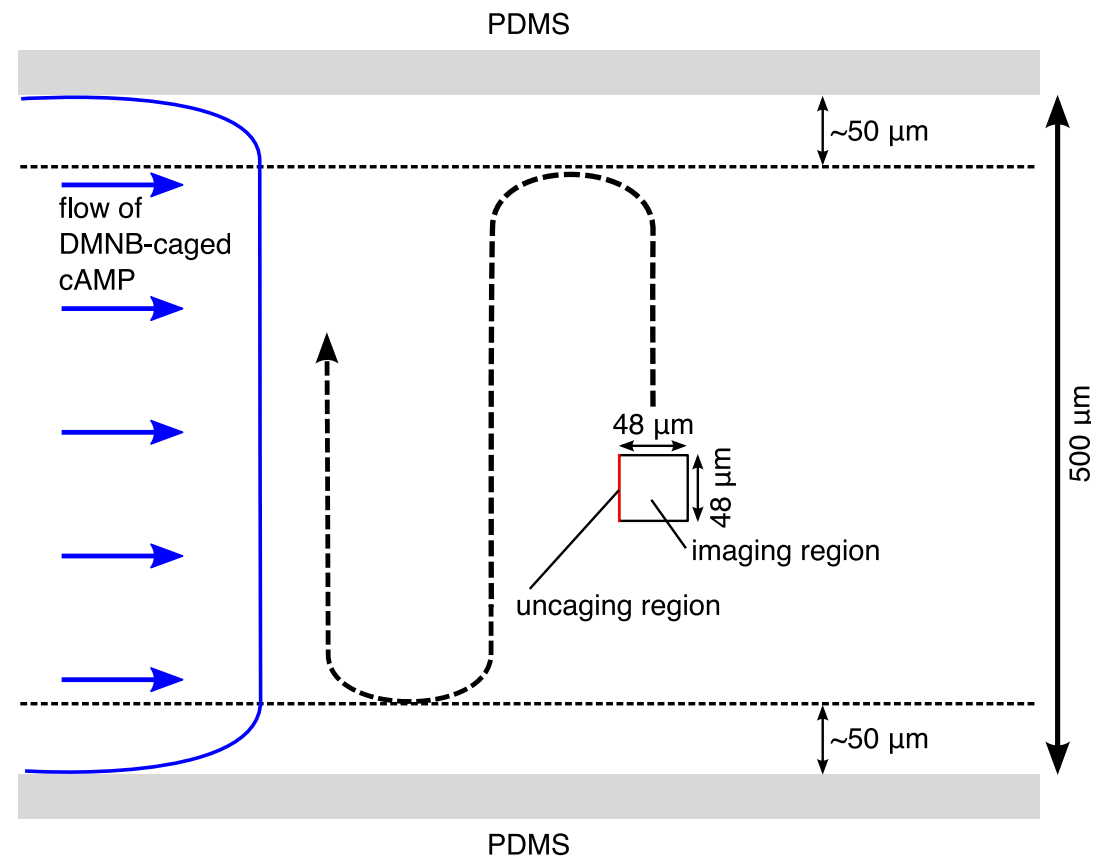

Figure 2.6.: Sketch of the microfluidic setup for periodic stimulation of $D$. discoideum. Prior to the experiment the microfluidic channel was filled with the cell suspension and the cells were allowed to settle down to the glass surface. The phosphate buffer was replaced with $10 \mu \mathrm{M}$ DMNB-caged cAMP. A flow with a mean velocity of $\vec{u}=120 \mu \mathrm{m} / \mathrm{sec}$ (see figure 2.4 for the velocity measurement) was engaged via the connected microfluidic pump (a schematic flow profile is drawn in blue). The imaging region (black square) and the uncaging line (red) were aligned via the Olympus Fluoview 1000 software as drawn in the figure. A single motile cell was centered within the region in advance of each experiment. To avoid boundary effects the region of interest was never placed closer than $50 \mu \mathrm{m}$ to the PDMS wall. After finishing one single experiment the region of interest was moved in a meandering manner upstream the flow, as indicated by the black dashed arrow.

\subsection{Image processing}

The recorded TIFF image sequence contains ideally, but not necessarily, one cell in the foreground, as well as the background and possibly fluorescent debris. Image processing was applied to distinguish fore- and background and to separate the cell of interest from other objects. The mask defining the foreground was created by locally thresholding the image using the Chan-Vese algorithm (Chan and Vese, 2001). This algorithm defines a curve, which distinguishes an outside (background) from an inside (foreground) region. It relies on minimizing the energy functional $\mathrm{F}$ :

$$
F\left(c_{1}, c_{2}, C\right)=\mu \text { Length }(C)+v \text { Area }(\operatorname{inside}(C))+\lambda_{1} F_{1}+\lambda_{2} F_{2} .
$$


$C$ gives the evolving curve, and $F_{1 / 2}$ are the integrals:

$$
F_{1 / 2}=\int_{\operatorname{inside}(C) / \operatorname{outside}(C)}\left|u_{0}(x, y)-c_{1 / 2}\right|^{2} d x d y,
$$

where $\mathrm{u}_{0}-c_{1,2}$ defines the difference between the foreground average and the average within/outside of the curve. The functional $F$ is minimized if both integrals $F_{1}$ and $F_{2} \approx 0$. This is only the case if the evolving curve $C$ equals the boundary of the foreground object, since both $u_{0}-c_{1}$ and $u_{0}-c_{2} \approx 0$. See figure $2.7 \mathrm{~A}-\mathrm{D}$ for a visualization of this algorithm. A $-\mathrm{C}$ show the three principal cases when the evolving curve not matching the boundary of the foreground $\left(C_{0}\right)$. In each of these cases at least one of the two integrals is larger than 0 and therefore the evolution continues until the evolving curve matches the foreground boundary (figure 2.7D). $\mu, v, \lambda_{1}, \lambda_{2}$ are fixed parameters, which weight the different terms and were set to $0.25,0,1,1$ respectively. These parameters are the standard parameters used by the original authors and were sufficient for the image segmentation purposes in this work. The Chan-Vese algorithm was implemented into a MATLAB routine by Getreuer (2010) ${ }^{11}$. The gained time sequence of all logical masks was labeled using MATLAB's bwlabeln.m function. This function assigns to all connected pixels (in x,y and time) the same number. Therefore all objects not labeled with the same number as the cell of interest were set to 0 . Hence, we obtained a mask for the cell of interest and furthermore we obtained the background ( $\overline{I_{\text {Bgnd }}}$, calculated as the average value of all pixels not belonging to the initial mask). The actin cortex is clearly distinguishable from the cytosolic region, as being a small band of high fluorescence intensity around the entire cell (see figure 2.7E). In response to a stimulus, the translocation of LimE-GFP increases the cortical and decreases the cytosolic fluorescence intensity. Consequently, the image has to be subdivided into a cortical and a cytosolic region. MATLAB's imerode.m function was used to erode the logical mask in order to obtain the mask of the cytosolic region. Image erosion shrinks the mask depending on a structuring element with which it is compared (Gonzales et al., 2004). The structuring element was chosen to be disk shaped of approximately $1.6 \mu \mathrm{m}$ (4 pixel) in radius, which defines the width of the cortical region. The image erosion is shown for a single cell example in figure 2.7F. Prior to averaging, all intensity values exceeding $C_{\text {mean }}+2 C_{\text {std }}$ (with $C_{\text {mean }}$ being the average value and $C_{\text {std }}$ the standard deviation of the cytosolic region) were added to the cortical region. This was necessary because the bright cortical region in some cases exceeded the diameter of the actual mask. The intensity values of each region are averaged and give the intensity values $\overline{I_{\text {Cor }}}$ for the cortical and $\overline{I_{\text {Cyt }}}$ for the cytosolic intensity respectively.

\footnotetext{
${ }^{11}$ The package can be downloaded from MATLAB's file exchange (FileID: 29743).
} 
A

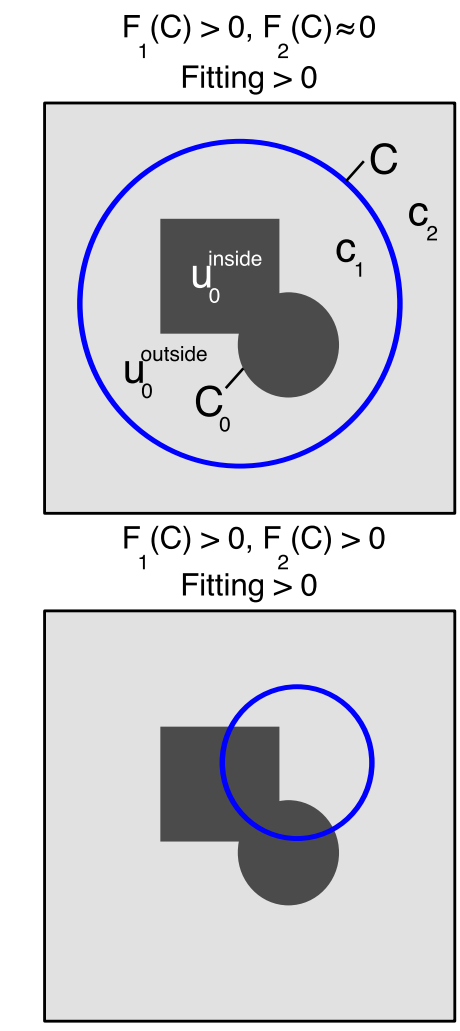

E

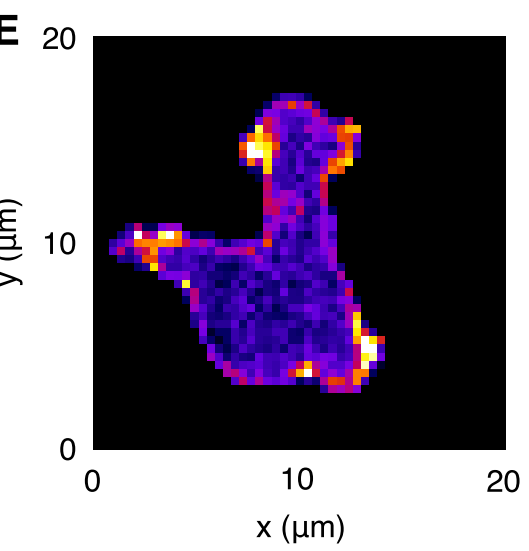

B

$F_{1}(C) \approx 0, F_{2}(C)>0$

Fitting $>0$

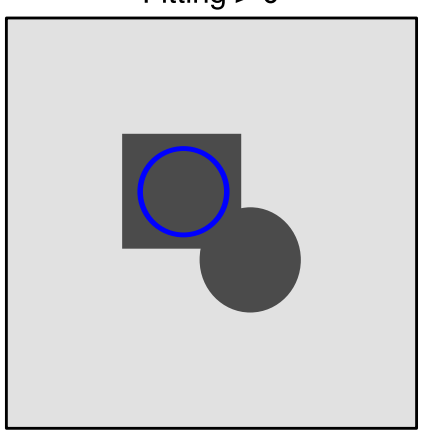

$\mathrm{F}_{1}(\mathrm{C}) \approx 0, \mathrm{~F}_{2}(\mathrm{C}) \approx 0$

Fitting $\approx 0$

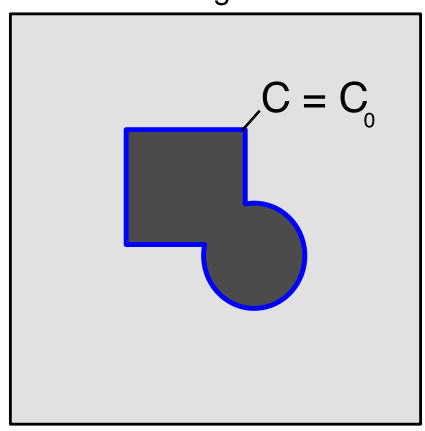

$\mathbf{F}$

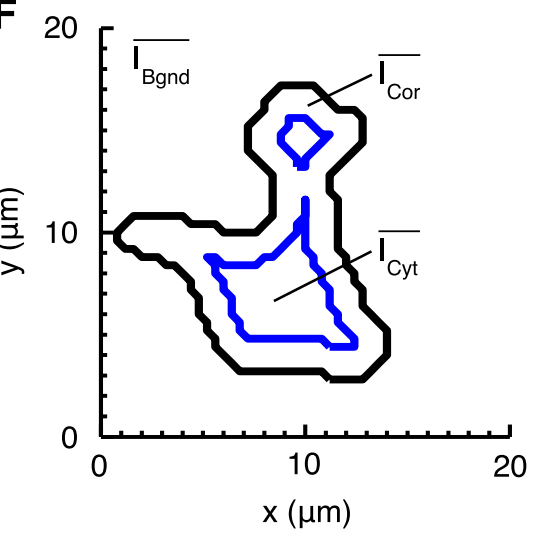

Figure 2.7.: Image segmentation using the Chan-Vese algorithm. (A-D) Visualization of the ChanVese algorithm (redrawn and adapted from Chan and Vese (2001)). The foreground object has a certain averaged intensity $u_{0}^{\text {inside }}$, which distinguishes it from the outside region (i.e. the background, $\left.u_{0}^{\text {outside }}\right)$. The evolving curve $C$ of the algorithm itself defines an inside and an outside region with their respective averaged values $\left(c_{1}, c_{2}\right)$. As long as $C$ does not equal $C_{0}$, the fitting term is larger than zero and the algorithm continues. (E-F) Image segmentation on a single cell example. The gray scale image (E, in false colors) is first distinguished from the background (using the Chan-Vese algorithm) and then subdivided (using image erosion) into a cortical (F, region between the black and the blue curve) and a cytosolic (F, inside the blue curve) region. Prior to computing the average intensity values of this region, all pixels exceeding an intensity of the average value plus twice the standard deviation were added to the cortical region. 


\subsection{Signal processing}

The averaged background intensity $\overline{I_{\text {Bgnd }}}$ was subtracted from every single averaged fluorescence value:

$$
I_{\mathrm{j}}^{*}(t)=\overline{I_{\mathrm{j}}(t)}-\overline{I_{\text {Bgnd }}}
$$

with $j=$ Cor, Cyt for the cortical and the cytosolic signal respectively. $I^{*}(t)$ gives the time signal to be processed and analyzed. The later application of the Fourier transform requires evenly spaced data. Due to hardware and software limitations the images were not sampled in equally spaced intervals. The jitter in data acquisition can reach up to $10 \%$ (i.e. $\Delta t=$ $0.9-1.1 \mathrm{~s}$ for a set value of $\left.\Delta t_{\text {set }}=1 \mathrm{~s}\right)^{12}$. A time series with equally spaced intervals is obtained by applying spline interpolation to the sampled signal (using MATLAB's spline.m function).

If no external stimulation was applied the interpolated signal was detrended by subtracting a line fitted to the signal. This had to be slightly adapted for signals, obtained in the presence of external stimulation. Now the interpolated signal can be thought of being composed of two parts. One part under stimulation and a part without external driving. A line was fitted exclusively to the region under stimulation. The line was then extended to the full length of the signal and the normalized and detrended signal was again obtained by subtracting the line from the original time series. Fitting the line only to the part under stimulation was necessary, since the mean and the variance are not constant over the full signal (due to the application of stimuli). For external driving experiments the interpolated and detrended signals were then averaged. Due to the different lengths of the individual timeseries ${ }^{13}$, the number of signals contributing to the average is not constant over time, but was always greater or equal to 3 .

Frequency analysis applied MATLAB's Fast Fourier Transform algorithm. If possible the signals were cut in period to prevent cut off artifacts. All signals were zeropadded to the respective next power of 2, prior to Fast Fourier Transform. The power spectra were estimated via the periodogram algorithm and used the MATLAB included spectrum.m function. The normalized power spectrum, i.e. the power spectrum divided by the sum of the full spectrum, could be used to automatically detect oscillations in a timeseries. A signal (or the respective fraction of it, if windowing was applied) was counted as oscillatory, if the normalized power of a certain frequency exceeded 0.1 (i.e. 1/10th of the total power) (Karra and Karim, 2009).

\footnotetext{
${ }^{12}$ The jitter was determined from the timevector measured by the Olympus Fluoview 1000 software itself. A software independent determination of the jitter suggested, that the measured timevector was accurate.

${ }^{13} \mathrm{D}$. discoideum is constantly moving and eventually leaves the region of interest after a certain amount of time.
} 
The unbiased autocovariance (ACV) was calculated as suggested by Flyvbjerg and Petersen (1989). It was used to determine the regularity of an oscillation, by the regularity of its zerocrossings. This also served as a detection criterion for oscillations (as described by Thornhill et al. (2003)), and was applied to automatically detect self-sustained oscillations (see chapter 4).

The period of an oscillation can be calculated directly from the zerocrossings of the autocovariance:

$$
\text { interval }=\frac{1}{2}\left(\overline{T_{p}} \pm \Delta T_{p}\right),
$$

with $T_{p}$ being the mean period and $\Delta T_{p}$, the variation of the period over the intervals. The corresponding standard deviation is given by $\sigma_{T_{p}}=2 \sigma_{\text {intervals. }}$. Thornhill et al. (2003) suggests to use the first 10 intervals (i.e. the first 11 zerocrossings) to determine the mean period and the standard deviation of it. The decision, whether an oscillation is present and regular relies on the spacing of its zerocrossings. The regularity is determined by

$$
r=\frac{1}{3} \frac{\overline{T_{\mathrm{p}}}}{\sigma_{\mathrm{p}}}
$$

If $r>1$ the signal (or the respective windowed part of it) is considered to be oscillatory. 
CHAPTER 3

\section{Actin cytoskeletal response to short-time stimuli}

The $D$. discoideum actin cytoskeleton is known to respond in a biphasic ${ }^{1}$ manner to a sudden upshift of external chemoattractant concentration (see Hall et al. (1988) for the cAMP and Yumura (1994) for the folic acid response). An increase in the polymerization of filamentous actin becomes apparent a few seconds after receiving the chemoattractant stimulus. The F-actin content of the cell reaches a sharp peak $5-10 \mathrm{~s}$ after the stimulus is applied. Within $20 \mathrm{~s}$ to $30 \mathrm{~s}$ the prestimulation F-actin content is recovered or even decreases below. A second extended phase of elevated F-actin level spans from 25 - 60 s (this period can extend to several minutes). The profile of this phase is broader, but it does not reach the same high F-actin levels as the first response phase. This behavior is sketched in figure 3.1A.

Analysis of labeled PH-domain proteins indicates the existence of the biphasic response on the level of the chemotactic reaction. The height of the second broad peak is significantly influenced by the action of PI3K and PTEN (Chen et al. (2003), Park et al. (2004)). Taking this into account the following explanation for the observed behavior has been suggested: The $D$. discoideum cell responds with a strong increase of actin polymerization to an external stimulus. The cell stops moving and rounds up, which is known as the cringe response (Futrelle et al., 1982). It lasts until the end of net F-actin depolymerization. Within the second, longer lasting phase lateral pseudopods are extended and the cell starts to move again (Janetopoulos and Firtel, 2008). Additionally it was observed that the actin response to a short pulse displays damped oscillatory properties (Vicker and Grutsch, 2008)2.

\footnotetext{
${ }^{1}$ The term biphasic here describes two phases within one signal, which have different properties.

${ }^{2}$ Experiments done with cells developed for 18 hours.
} 
Here LimE-GFP is used to indirectly label the polymerization and branching of filamentous actin. The time profile of the cortical and the cytosolic LimE-GFP fluorescence in response to a step input of cAMP is shown in figure 3.1B. The timescale of the step and the pulse responses were calculated as the crossing of the cytosolic fluorescence signal with the lower confidence boundary. This boundary is derived from the confidence intervals of the prestimulation signal. The measured timescales of the step response are comparable to those found in previous experiments (see Hall et al. (1988), Chen et al. (2003), Park et al. (2004), Lee et al. (2010)). The initial cytosolic depletion starts $3 \mathrm{~s}$ after receiving the stimulus and lasts for $16 \mathrm{~s}$. The next minimum starts $32 \mathrm{~s}$ after the stimulus is applied and continues for a further $20 \mathrm{~s}$. It should be noted that the fluorescence profile does not give an information how the actual filamentous actin content of the cell changes over time, but rather the translocation of LimE-GFP to areas of increased actin polymerization. It was previously reported that the second peak decreases in height with increasing developmental time of the cell (Park et al., 2004). Within our experiments ( $7-9$ hours starvation time) the second phase is still significant (i.e. below the lower confidence boundary).

The flow photolysis technique allowed us to create cAMP pulses as short as $1.5 \mathrm{~s}$, which served here as approximate $\delta$ functions. The response of the LimE-GFP fluorescence to this short-time cAMP stimulus (figure 3.1C) indicates cytosolic depletions that are shorter than their step response counterparts. Consequently the second minimum starts and ends earlier. Additionally, an overshoot of cytosolic LimE-GFP fluorescence is observed $19-20 \mathrm{~s}$ after application of the stimulus. This can be explained as follows: Net depolymerization decreases the amount of F-actin (and hence LimE-GFP) in the cell cortex below the prestimulus level. The LimE-GFP is released to the cell cytosol resulting in a fluorescence overshoot $^{3}$. The averaged pulse response signal shows similarities to a damped oscillation. Subdividing the response into intervals (again determined by the crossing of the signal with the lower confidence boundary) shows an analogy to a long-short alternans; the two long cytosolic depletions are interspaced with short fluorescence recoveries (indicated by the red dots in figure 3.1C). However, alternans, as described for the cardiac action potential (see Weiss et al. (2006) for a review), refers to the alternation of the pulse length with respect to the external pacing. One long response to a single stimulus is followed by a short response to the subsequent stimulus. Regarding the pulse response timescale, the first full period is $22 \mathrm{~s}$ long. This includes the $3 \mathrm{~s}$ delay for the LimE-GFP translocation to become visible. The second, strongly damped, period increases in duration to $25 \mathrm{~s}$.

\footnotetext{
${ }^{3}$ This overshoot is different from the in vitro and in vivo actin polymerization overshoot (Pantaloni et al. (1984), Buzan and Frieden (1996), Goley and Welch (2006), Brooks and Carlsson (2010)), which was recently reviewed in Carlsson (2010).
} 

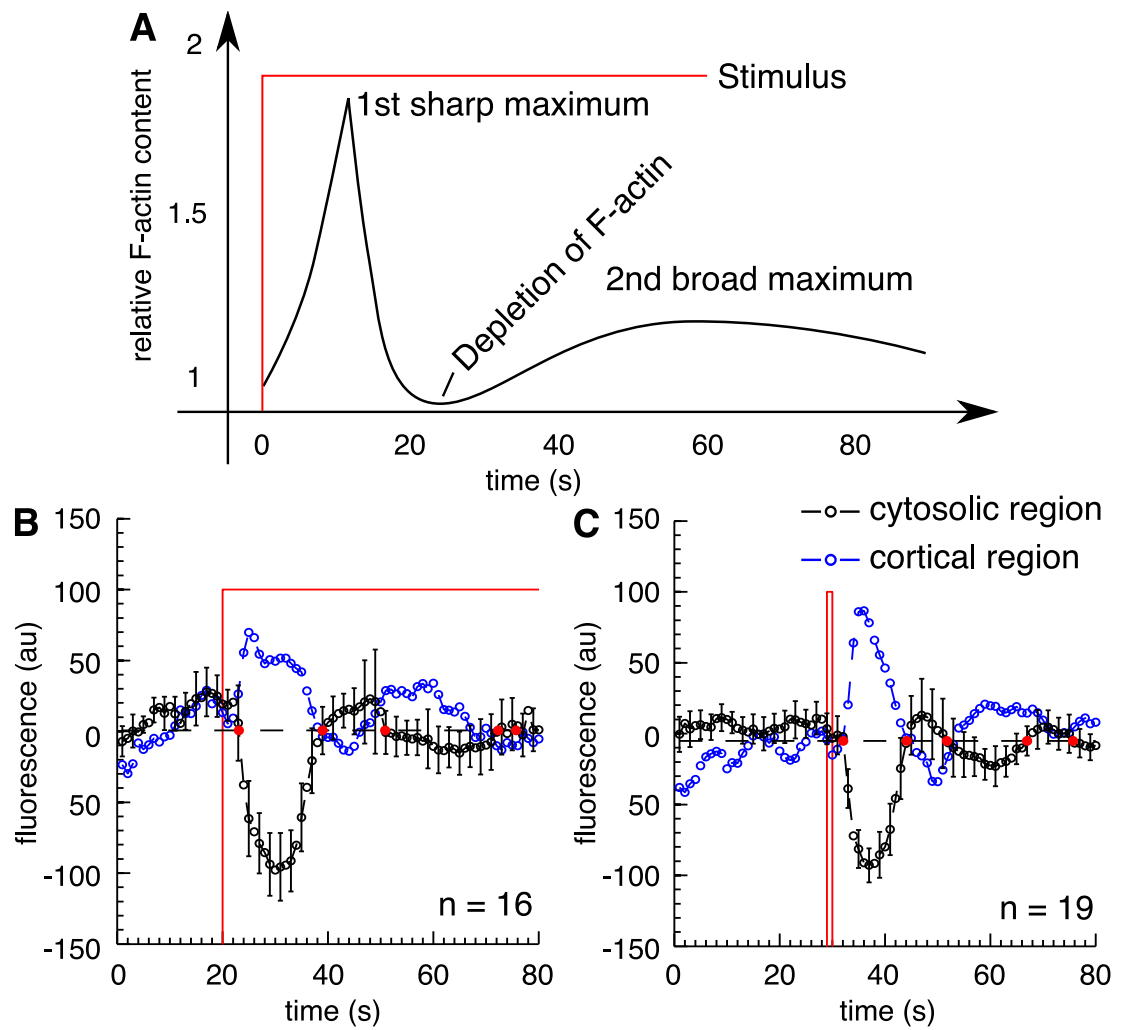

Figure 3.1.: Response behavior of the filamentous actin to a sudden chemoattractant stimulus. (A) Sketched time profile of the biphasic response (Hall et al. (1988), Yumura (1994)). The absolute content of the filamentous actin in the cell in response to a 1 min stimulation of cAMP increases initially, peaking after 5-10s. This peak decreases dropping to the prestimulation value or even below. After $30 \mathrm{~s}$, a second broader maximum is observed. (B) In the experiments described in the subsequent section LimE-GFP labels the filamentous actin. In response to a step input (red line) this label shows a biphasic fluorescence response profile. This is particularly prominent in the cytosolic fluorescence signal. However, it should be noted that in vivo confocal micrographs of the LimE-GFP molecule indicate the distribution of F-actin, but do not contain enough information to determine the entire F-actin content in the cell (as sketched in (A)). When the input time is shortened to $1.5 \mathrm{~s}$ the response profile resembles a damped oscillation (C). In (B) and (C) the response onset and end are defined by the intersection of the fluorescence signal with the dashed line. The dashed line gives the lower averaged confidence interval. This interval is the average of all individual confidence intervals (black errorbars) before the stimulus is applied. The response onset and end are indicated by red dots. 


\subsection{Observed temporal patterns}

Cell to cell variability is an intrinsic property of biological systems. Figure 3.2 summarizes the oscillatory patterns for a number of different cells. Fluctuations of the cytosolic LimEGFP fluorescence without sustained oscillations were observed in $90 \%$ of all recorded cells (total number of experiments $\mathrm{N}=271$, figure 3.2A shows the overlay of all non-oscillating controls). Fluctuations arise due to the dynamic nature of the actin cytoskeleton and also because the $D$. discoideum cell is constantly moving. Furthermore, it should be noted that the imaged area of the cytosolic region (as well as the cortical region) was not constant over time. Due to its movement, the cell changes shape and as a result the imaged confocal crossection also changes. A minor fraction of cells showed regular oscillatory patterns of LimE-GFP fluorescence, one example of this is shown in figure 3.2B. The amplitude of the self-sustained oscillation is comparable to the magnitude of the fluctuations. The spectrum of the averaged signal from figure 3.2A is compared to the spectrum of figure $3.2 \mathrm{~B}$ in figures $3.2 \mathrm{C}$ and $\mathrm{D}$ respectively. The non-oscillatory spectrum resembles that for brownian noise (1/f decaying frequency spectrum). The frequency and amplitude have a characteristic distribution, this is discussed in more detail in section 3.4. Finally, as described above, a single short-time pulse leads to a damped oscillatory profile ${ }^{4}$. However different degrees of damping were observed for the same experimental conditions as shown by the two single cell examples (figures 3.2E and F). These figures show a time trace characteristic of the majority of the pulse responses. The signal is strongly damped and decays after the second fluorescence depletion event. Weak damping is also observed, although rarely (less than $10 \%$ of all pulse responses). An example of this is given in figure 3.2F. The pulse response is visible for at least four full periods. The damping of figures 3.2E and $\mathrm{F}$ is compared in figures G and H. To analyze the response, the signal is Savitzky-Golay filtered (black dots) and fitted with an exponentially decaying single frequency sinusoid (blue line). The orange dashed envelope visualizes the damping. The timescale of the weakly damped response is shorter (by 3 to $4 \mathrm{~s}$ when comparing the first cytosolic depletion) than the more strongly damped response. Looking now at the average of the response for all recorded cells, the following results are found: The onset of the response starts at $t=3.4 \pm 1.50 \mathrm{~s}$. The subsequent depletion of cytosolic fluorescence intensity lasts until $\mathrm{t}=13.6 \pm 2.67 \mathrm{~s}$. The entire length of this cycle is $22.8 \mathrm{~s}^{5,6}$. In 8 out of 18 cases, the overshoot of cytosolic fluorescence intensity is significantly different from the prestimulation average.

\footnotetext{
${ }^{4}$ Both examples given in figure $3.2 \mathrm{E}$ and $\mathrm{F}$ belong to the non-autonomous oscillating fraction of cells.

${ }^{5}$ The full period includes the $3 \mathrm{~s}$ lasting response delay.

${ }^{6}$ The length of the full period is determined from the averaged pulse response signal.
} 

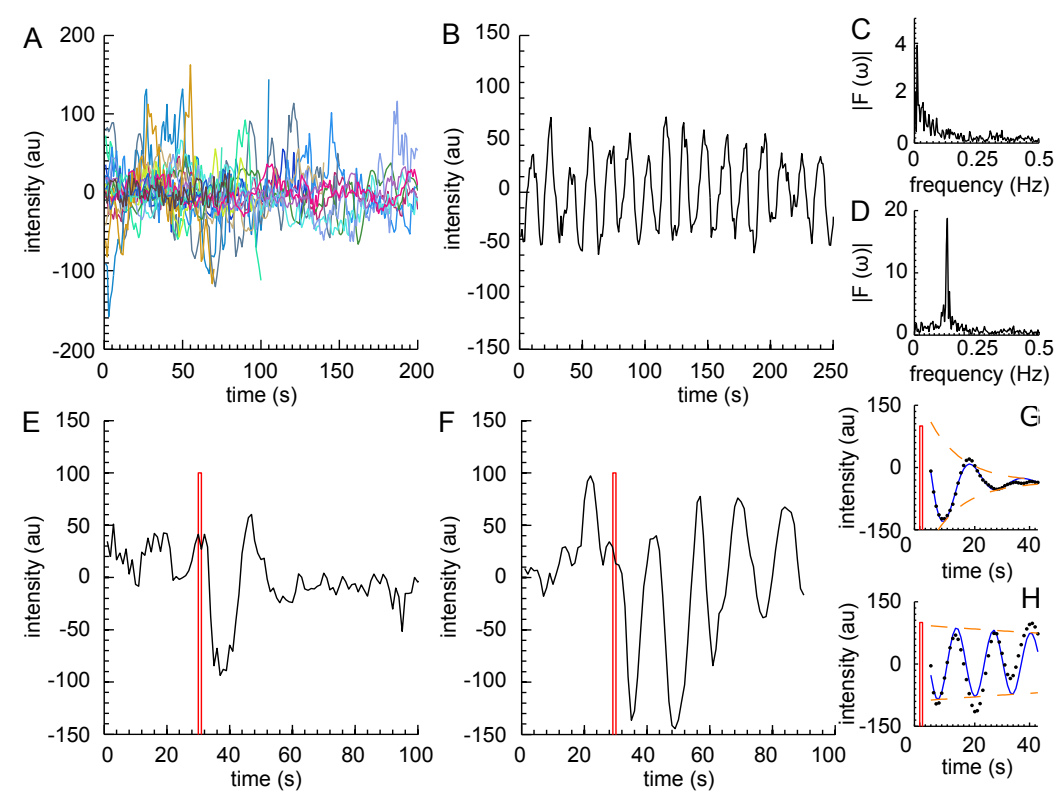

Figure 3.2.: Variety of the observed oscillatory patterns. (A) Overlay of cytosolic LimE-GFP fluorescence profiles of cells not exposed to external cAMP stimulation. These non-oscillatory patterns (C shows the spectrum of the corresponding averaged signal) make up more than $90 \%$. In rare cases $(<10 \%)$ an ongoing oscillation is observed. One single cell example is plotted in (B) and its respective spectrum in (D). The observed damped oscillatory behavior showed a remarkable cell to cell variety as visualized by two single cell examples in (E) and (F). (G-H) Different damping strengths of the impulse responses shown in (E) and (F). A single frequency and exponentially decaying sinusoid (blue) is fitted to the filtered and interpolated signal (black dots). The envelopes (orange dashed) describe the strength of the damping.

\subsection{Characterization of the periodic response}

To substantiate the existence of an intrinsic timescale, single $D$. discoideum cells were exposed to periodic sequences of cAMP pulses. Stimulus periods ranging from $\mathrm{T}=4 \mathrm{~s}$ to $\mathrm{T}=60 \mathrm{~s}$ were applied to systematically explore the response of the filamentous actin network. We chose $\mathrm{T}=4 \mathrm{~s}$ as the lower limit to ensure that the cAMP released during a single uncaging event was washed away by the microfluidic flow before the next pulse was applied. An upper limit of $60 \mathrm{~s}$ was selected since the response to a single pulse is seen to fully decay to the base level over this time period. The following subsections are distinguished into low $(-8 \mathrm{~s})$, medium $(10-20 \mathrm{~s})$ and high $(>20 \mathrm{~s})$ period external forcing. The discrimination is done on the basis of the pulse response timescale; the application time of the second pulse in the pulse train is compared to the phase of the pulse response at that time ${ }^{7}$.

\footnotetext{
${ }^{7}$ For instance low and medium periods are distinguished, whether the second pulse is applied before or after the pulse response reached its fluorescence minimum.
} 


\subsubsection{Low period external forcing}

External driving periods of $\mathrm{T}=4-8 \mathrm{~s}$ are designated as low period external forcing. For these timescales the second pulse within the spike train is applied before, or as, the resulting fluorescence intensity reaches its minimum (see figure 3.3A). The cytosolic LimE-GFP response to a $4 \mathrm{~s}$ input function is shown in figure 3.3B. A prolonged initial depletion is observed before a recovery to a steady state response with small oscillatory fluctuations. However, a clear peak in the frequency spectrum is observed at $\mathrm{f}=0.25 \mathrm{~Hz}$ (figure 3.3F). To investigate, whether LimE-GFP really translocates on this fast timescale a strain expressing cytosolic $\mathrm{GFP}^{8}$ was exposed to $4 \mathrm{~s}$ external driving (figure 3.3C). A similar steady state response is observed however, there is no initial fluorescence depletion due to the absence of the LimE-protein. The similarity of the LimE-GFP and cytosolic GFP signals can be seen from their spectra (figures $3.3 \mathrm{~F}, \mathrm{G}$ ), which both have peaks at $\mathrm{f}=0.25 \mathrm{~Hz}$ and with comparable amplitudes. This therefore gives a lower limit for any detection made by the LimE-GFP label. This property could be introduced by fast and small changes of the optical properties in the cell (Dewitt et al., 2009) or could indicate a minor association of the GFP itself to the actin cytoskeleton as discussed by Pang et al. (1998), but denied by Heinrich et al. (2008). The $6 \mathrm{~s}$ response also shows an initial prolonged depletion (figure 3.3D). Consequently the normalized ${ }^{9}$ initial responses of both the $4 \mathrm{~s}$ and $6 \mathrm{~s}$ timeseries were compared to the step and the pulse response (figure 3.3J). Doing this makes it clear that the cell response is similar to a step input or, in other words, the cell is not able to distinguish these fast input periods from a continuous input. The $D$. discoideum cell acts as a low pass filter for the input signal, with the $1 / 4$ and $1 / 6 \mathrm{~Hz}$ frequencies being blocked ${ }^{10}$.

For periodic forcing with $\mathrm{T}=8 \mathrm{~s}$ (figure 3.3E), the initial response of the cell is prolongued, but now a transient type of behavior is observed. The time between the stimuli is long enough that the cell can distinguish subsequent pulses. This gives rise to an oscillatory pattern in the steady state response that is much more obvious that forcing at $4 \mathrm{~s}$ and $6 \mathrm{~s}$ periods. However, the spectral amplitude (figure 3.3I) is still comparable to the cytosolic GFP control. The autocovariances of the response signals to 8 and $10 \mathrm{~s}$ inputs show the development of a regular periodic response (figures $3.3 \mathrm{~K}-\mathrm{M}$ ). The spacings of the ACV zerocrossings approach an averaged value equal to $1 / 2$ of the oscillation period, indicating a regular and single frequency oscillation. The onset of a periodic oscillation is therefore located for stimuli input between $8 \mathrm{~s}$ and $10 \mathrm{~s}$ periodic cAMP input (figures $3.3 \mathrm{~L}-\mathrm{M}$ ).

\footnotetext{
${ }^{8}$ The GFP is overexpressed as it is under the control of an Act15 promotor, but is not bound to another protein. Therefore, it freely diffuses through the cytosol.

${ }^{9}$ The signals were normalized by dividing the signal by their largest absolute value.

${ }^{10}$ Interestingly, the immediate response of the cell to cAMP, i.e. until the maximal depletion of cytosolic fluorescence is reached, is independent from the temporal profile of the applied stimulus.
} 


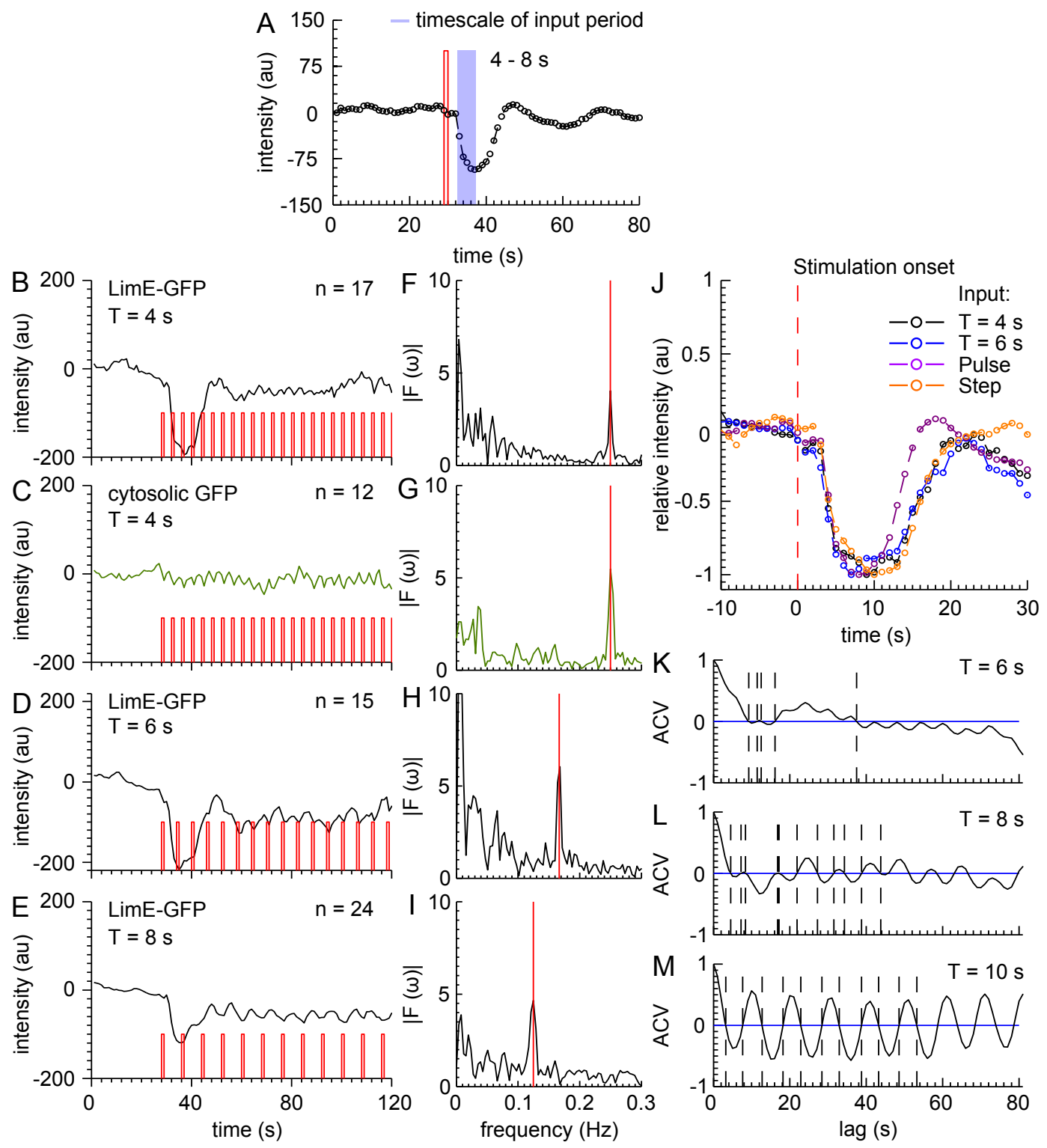

Figure 3.3.: Periodic stimulation at low external forcing. (A) The cytosolic response (black) to a pulse lasting $1 \mathrm{~s}$ (red). Marked in blue is the phase of the response at which the subsequent stimulus pulse is applied for $\mathrm{T}=4-8 \mathrm{~s}$. This occurs before the cytosolic region reaches the first and largest fluorescence minimum. (B-E) Fluorescence profiles of a LimE-GFP cell (B,D,E, black line) and a cytosolic GFP expressing cell (C, green line) to periodic stimulation (red) at periods ranging from $4 \mathrm{~s}$ to $8 \mathrm{~s}$. The corresponding frequency spectra of the steady state responses are given in (F-I). Note the similarity of the $4 \mathrm{~s}$ cytosolic GFP and the $4 \mathrm{~s}$ LimE-GFP response, which determines the detection limit of this label. The response to $4 \mathrm{~s}$ and $6 \mathrm{~s}$ periodic stimulation is similar to a step response. (J) Comparison of the response to a single pulse, a step function and $4 \mathrm{~s}, 6 \mathrm{~s}$ periodic stimuli. Clearly the response to a single pulse (purple) reaches the prestimulation value earlier than the 3 other responses. The onset of the different stimuli is marked by the red dashed line. (K-M) The steady state response becomes more regular as the stimuli period increases from $8 \mathrm{~s}$ to $10 \mathrm{~s}$. This is shown by the autocovariances (black) of the respective steady state signals and the spacing of their zerocrossings (indicated by horizontal dashed lines). The onset of an oscillatory response occurs for stimuli with a periodicity between $8 \mathrm{~s}$ and $10 \mathrm{~s}$. 


\subsubsection{Medium period external forcing and transient characterization}

Medium periodic forcing of the $D$. discoideum cells is defined as stimuli with periodicities ranging from 10 to $20 \mathrm{~s}$. For these periodicities the second pulse of the input spike train is applied during the net depolymerization of the F-actin, i.e. the phase in which LimE-GFP gets retranslocated to the cytosolic region and increases its fluorescence. The cytosolic fluorescence responses are given in figures 3.4B - E and their respective frequency spectra in figures $3.4 \mathrm{~F}-\mathrm{I}$. A clear increase in the amplitude of the oscillation is observed as the periodicity of the stimuli is increased from $T=10 \mathrm{~s}$ to $\mathrm{T}=20 \mathrm{~s}$. The longer forcing periods allow a stronger response to develop. Two other properties of the response to medium external forcing are interesting. First, as mentioned for $\mathrm{T}=8 \mathrm{~s}$, a transient behavior is observed for $\mathrm{T}=10 \mathrm{~s}$. This transient behavior vanishes when the system is driven by stimuli with longer periods (figure 3.4J). Each individual response (i.e. the timesignal within an interval determined by two consecutive stimuli) is correlated with its subsequent response. When a steady state oscillation is reached the correlation coefficient approaches 1; each response is almost identical to the next. The first and second responses for $\mathrm{T}=8 \mathrm{~s}$ are anticorrelated, while the correlation coefficient between the second and third responses is slightly positive. This indicates that for $\mathrm{T}=8 \mathrm{~s}$ the cytosolic signal needs $2-3$ full cycles to reach a steady state. This is less pronounced but still detectable for $10 \mathrm{~s}$ and $12 \mathrm{~s}$ periodic forcing, which require two and one periods respectively to reach a steady state. Transient behavior is not detectable for response periods with $\mathrm{T} \geq 15 \mathrm{~s}$.

The second interesting phenomenon within this range of input periods is a shift of the baseline. Looking at the responses for $\mathrm{T}=10 \mathrm{~s}$ and $\mathrm{T}=12 \mathrm{~s}$ (figures $3.4 \mathrm{~B}-\mathrm{C}$ ) one can see that the steady state response oscillates around a basevalue lower than the prestimulation value. To validate this, the first 3 periods of the steady state signal are highlighted (figure $3.4 \mathrm{~K}$ and $\mathrm{L}$, orange) and compared to the averaged confidence intervals (black dashed lines) as calculated from the individual confidence intervals of the prestimulation signal. It appears that the mean of the steady state is lower than the prestimulation mean. This is less prominent in the response signal when $\mathrm{T}=20 \mathrm{~s}$ (figure $3.4 \mathrm{~L}$ ). Here an overshoot of the cytosolic fluorescence intensity above the upper confidence boundary is observed. This phenomenon can be explained in the context of the pulse response timescale. Including the time delay of $3 \mathrm{~s}$ observed between the initial stimulus and the subsequent cell response, the consecutive stimulus has passed through the signal processing system, while LimE-GFP relocates to the cytosol. Two counteracting processes are occurring: (1) LimE is relocating to the cytosol (the late phase of the response to the first stimulus) and (2) LimE is localizing to the cell cortex in response to the second stimulus. This leads to a lower steady state concentration of LimE-GFP in the cytosol and therefore to a decreased averaged cytosolic fluorescence. 


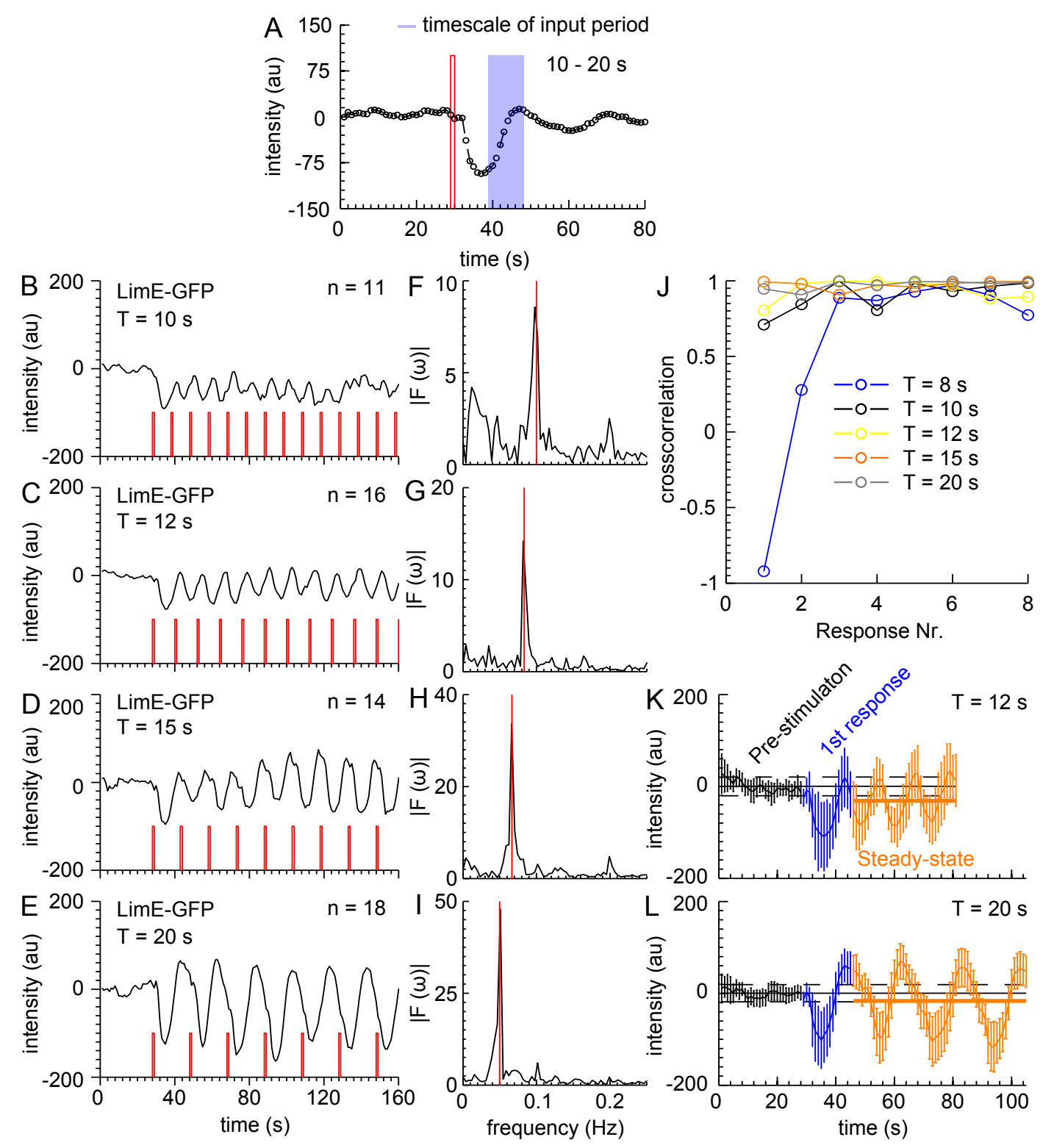

Figure 3.4.: Periodic stimulation at medium periodic forcing. (A) Here the applied input periods range from $\mathrm{T}=10 \mathrm{~s}$ to $\mathrm{T}=20 \mathrm{~s}$. The second pulse of each input spike train is applied in the depolymerization regime (indicated by blue area). The cytosolic LimE-GFP responses are given in (B-E) and their respective frequency spectra in (F-I). Note the changing scale from F to I, due to the increase in the amplitude of the oscillatory response. In B and C as well as in figure 3.3D transient behavior is visible prior to the steady state response being reached. To characterize this transient behavior each individual response is crosscorrelated with the next. This correlation as a function of the response period is plotted in $(\mathrm{J})$. Note that this plot gives the time in terms of periods of the input signal and not in seconds. The steady state response for $\mathrm{T}=10 \mathrm{~s}$ and $\mathrm{T}=12 \mathrm{~s}$ appears to oscillate around a mean value, lower than the prestimulus mean. This is compared with the response for longer stimuli periods in $(\mathrm{K})$ and $(\mathrm{L})$. The prestimulation response is indicated by a black line. The errorbars give the confidence intervals. From these the lower and upper averaged confidence boundary is calculated (black horizontal dashed lines). Comparing the response to the first stimulus (blue) and the steady state response (orange) to the prestimulation value, it appears that for $\mathrm{T}=12 \mathrm{~s}$ the response does not overshoot the prestimulation average, while for $\mathrm{T}=20 \mathrm{~s}$ it does. 


\subsubsection{High period external forcing}

When the periodicity of the stimuli is shifted to values greater than $\mathrm{T}=22 \mathrm{~s}$ (the estimated period of the pulse response), the second pulse of the input pulse train hits during or after the second and strongly damped response of the actin cytoskeleton (region is marked blue in figure 3.5A). Again, for comparison, the respective cytosolic response signals are given in figure $3.5 \mathrm{~B}-\mathrm{E}$ and their spectra in figures $3.5 \mathrm{~F}-\mathrm{I}$. Here the increasing stimuli period allows the cell to pass through more parts of the single pulse response, before the next stimulus is applied. Consequently, a second smaller translocation of LimE-GFP to the cortex becomes apparent within one period of the pulse train. This is visible as a little kink in the response for $\mathrm{T}=25 \mathrm{~s}$, which grows as the periodicity of forcing increases in length. To understand this in greater detail the cytosolic response signal for $\mathrm{T}=35 \mathrm{~s}$ was time-delay-embedded in three dimensions (figure 3.5J). In phase space one can see that the system spirals back towards the fixed point after being pushed outwards by the external stimulus. However, before reaching the fixed point the system is again kicked to the outermost position of the cycle (indicated by the red arrow) and the spiraling process restarts. The periodicity of forcing therefore sets the time on which the system is reseted and restarts with a new first response. The second smaller response leads to the appearance of the second harmonic frequency in the spectrum and a decrease in the amplitude of the spectrum at the input frequency (figure 3.5F - I, red line). This behavior can be assigned as period halving (figure $3.5 \mathrm{~K}-\mathrm{M}$ ). The ACV contains the same amount of information as the frequency spectrum, but allows the period halving to be visualized easily from the ACV zerocrossing pattern (black dashed lines). For $\mathrm{T}=25 \mathrm{~s}$ and $\mathrm{T}=30 \mathrm{~s}$ the averaged period of the response, given by the zerocrossings is close or equal to that of the input period. From $\mathrm{T}=30 \mathrm{~s}$ to the $\mathrm{T}=35 \mathrm{~s}$, the correlation of the second response with the first increases and crosses the zero line. A response period of $17.4 \mathrm{~s}$ is found; the response period halves with respect to the input period. However, the period 2 type of behavior still remains. A strong cytosolic depletion alternates with the weaker depletion of fluorescence intensity.

For $\mathrm{T}=60 \mathrm{~s}$ the resulting cell response is observed to decay. This is reasonable as the input periodicity timescale is now longer than the pulse response timescale. The actin cytoskeletal system can therefore reach its prestimulation state before the next pulse is applied. Consequently the response is only marginally different to the cytosolic response for $\mathrm{T}=60 \mathrm{~s}$ external driving. It should be noted that LimE-GFP labels only the state of the filamentous actin. Other proteins or systems, for instance the adenylate cyclase ${ }^{11}$, may not recover their prestimulation activity within $60 \mathrm{~s}$.

\footnotetext{
$\overline{{ }^{11} \text { The adenylate cyclase is part of the cAMP }}$ relay and remains active up to 2 minutes after receiving a stimulus.
} 


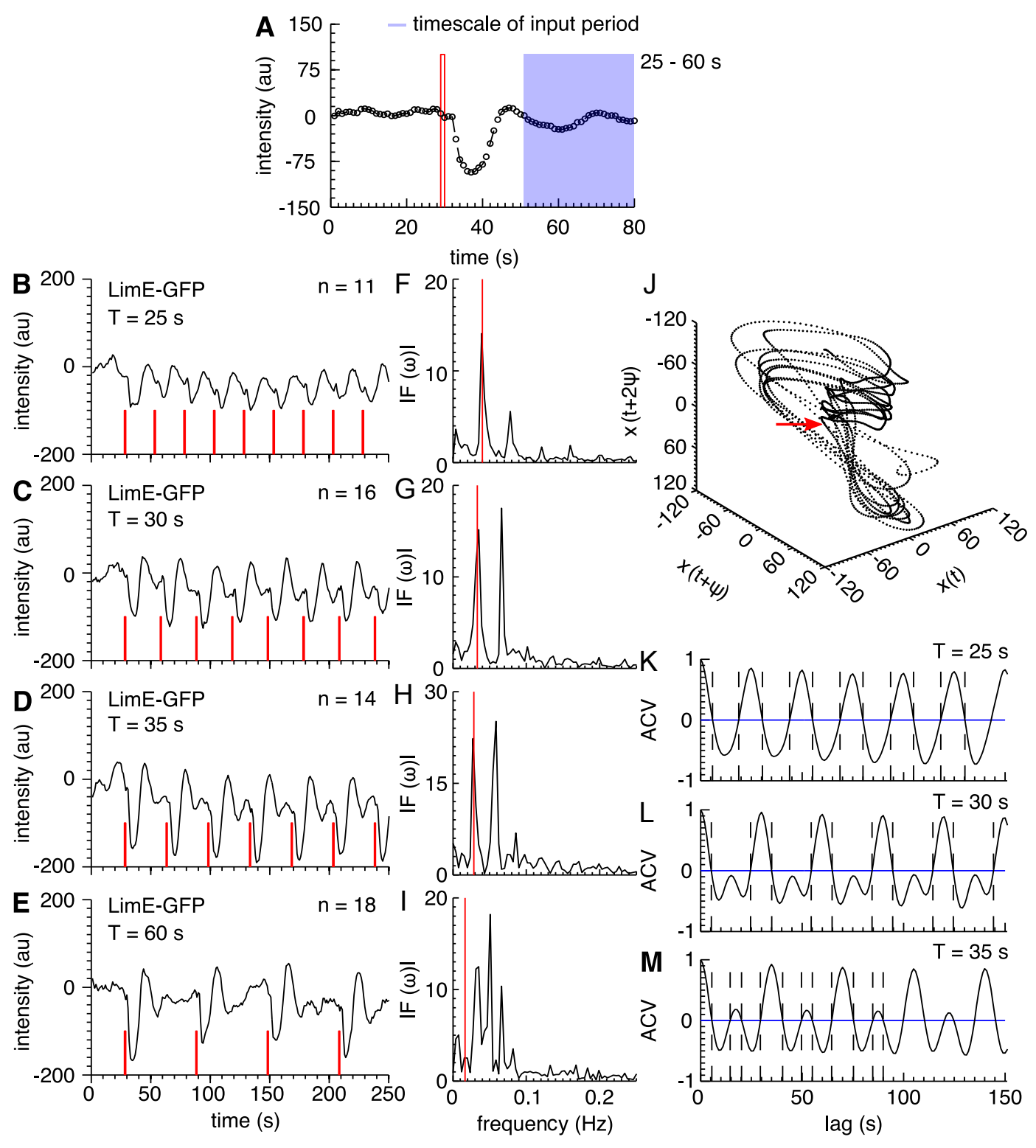

Figure 3.5.: Periodic stimulation at high periodic forcing. (A) Stimuli periods ranging from $T=25 \mathrm{~s}$ to $\mathrm{T}=60 \mathrm{~s}$. The approximate period of the pulse response is $22 \mathrm{~s}$ and therefore the second pulse of the pulse train hits the cell during or after the second damped phase of its response. (B-E) The cytosolic response profiles (black) are plotted. The input function is indicated by the red lines. (FI) show the spectra of the steady state responses. (J) Three-dimensional time-delay embedding of the response for $\mathrm{T}=35 \mathrm{~s}$. $\Psi=2 \mathrm{~s}$ was chosen for best unfolding of the underlying attractor. The signal was Savitzky-Golay filtered and interpolated prior to embedding. (K-M) Zerocrossing pattern (black dashed) of the autocovariance functions (black solid) for $\mathrm{T}=25 \mathrm{~s}-35 \mathrm{~s}$. Period halving of the response is observed for $\mathrm{T}>30 \mathrm{~s}$. 


\subsubsection{Characterization of the oscillatory properties}

A summary of the results characterizing the oscillation behavior is given in figure 3.6. Figure $3.6 \mathrm{~A}$ shows the development of the response spectrum as the stimulus period $\mathrm{T}$ increases. The magnitude of the frequency spectrum is given as gray scale values. One can see the rise and decay of the first and second harmonics as the stimulation period increases. Figure 3.6B shows the evolution of the dominant peaks in the frequency spectrum over time. The dominant mode is marked in black and the second largest in red. The first harmonic frequency is the dominant mode for $\mathrm{T} \geq 8 \mathrm{~s}$. For $\mathrm{T} \geq 20 \mathrm{~s}$, a second harmonic appears in the spectrum. This second harmonic becomes the dominant mode for $\mathrm{T} \geq 30 \mathrm{~s}$ input. A linear fit is applied to the first and the second harmonics to obtain the slopes, which are equal to 1 and 0.55 respectively ${ }^{12}$. The dominant mode switches again for $\mathrm{T}=60 \mathrm{~s}$, where the greatest response is seen for the third harmonic. The amplitude of the dominant mode is highest for a forcing period $\mathrm{T}=20 \mathrm{~s}$. The full amplitude response is given in figure 3.6C. Keep in mind that the input amplitude (concentration of cAMP) and the response amplitude (fluorescence light intensity) cannot be compared directly. Only the different response amplitudes with respect to each other can be compared.

In the above experiments, all parameters were kept constant while the period of forcing was changed. The input of each new cAMP pulse is seen to drive the system back to its starting position. If this (including the delay due to signal processing) occurs within the fluorescence recovery and depolymerization overshoot, then the ongoing recovery and external forcing counteract each other. Consequently, the maximal response was observed for cells stimulated every $20 \mathrm{~s}$, since this is close to the previously determined pulse response time of approximately $22 \mathrm{~s}$ (see section 3.1). For $\mathrm{T}=20 \mathrm{~s}$ the subsequent stimulus is applied at the same time as the overshoot of cytosolic intensity appears. However, the signal needs at least $3 \mathrm{~s}$ to pass through the signal processing system. It starts to engage actin polymerization right in the moment that the cell would enter the second phase of the pulse response. The driving leads the cell to enter a new first cycle and the next pulse is applied at the right moment to keep the system at the outermost cycle. Instead of spiraling inwards, a limit cycle with the maximal distance to the fixed point is established.

The averaged amplitude could be effected by cell to cell variability; slightly phase shifted single signals would lead to a smaller averaged amplitude. However, the variances over time of all response signals are comparable ${ }^{13}$. Hence we can speak of a resonance of the actin cytoskeleton and the chemotactic system at $\mathrm{T}=20 \mathrm{~s}$.

\footnotetext{
${ }^{12}$ This result is expected. For the first harmonic the response equals the input period and for the second harmonic it equals half the input period.

${ }^{13}$ The variance would increase if the single signals were phase shifted with respect to each other.
} 

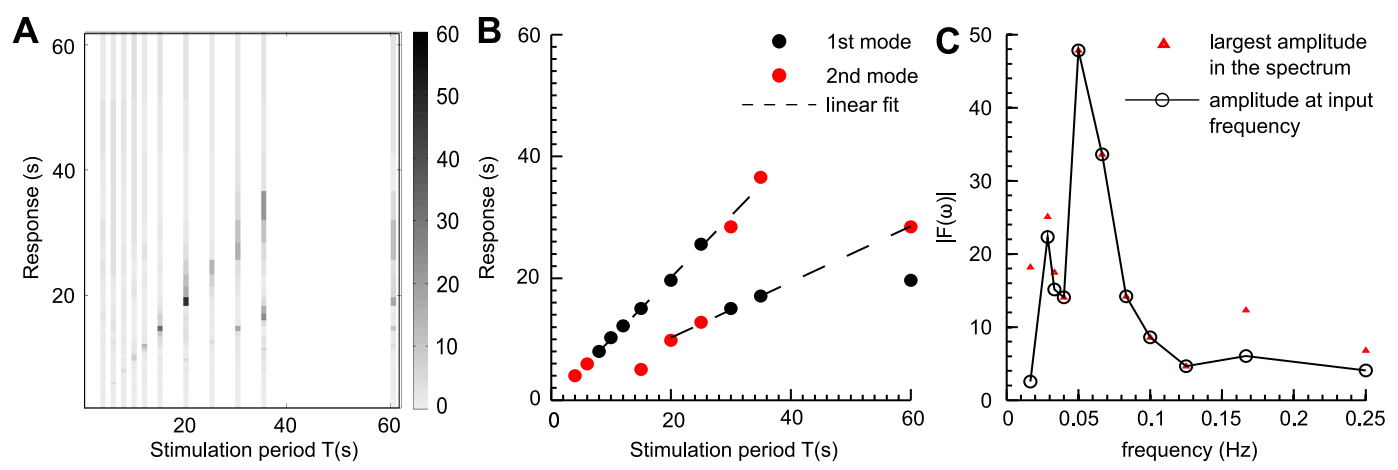

Figure 3.6.: Characterization of the oscillatory properties for all external forcing experiments. (A) Fourier spectra of the cytosolic response signals to periodic stimulation. This period of the uncaging experiments is given on the $\mathrm{x}$-axis, while the $\mathrm{y}$-axis shows the response of the cell (i.e. 1/f). The magnitude of the Fourier spectrum is gray scale coded. The two largest modes are plotted in (B). The dominant mode in the Fourier spectrum is colored in black and the second largest mode in red. A linear fit is applied to the first and the second harmonic (dashed line). As expected the slope is $\approx 1$ for the first harmonic and $\approx 0.55$ for the second. Note, that for short forcing periods the dominant modes are hidden by low frequent noise. (C) The largest amplitudes of each response frequency spectrum are plotted as a function of stimulation period (red triangles). This is to be compared with the amplitude of the spectrum at the respective input frequency, i.e. the frequency of the external forcing (black line and circles). If the two overlay each other $(0.05$ to $0.125 \mathrm{~Hz})$ the largest mode occurs at the input frequency. This is lost for lower frequencies as the dominant mode switches to the second harmonic (can also be seen in (B) between $25 \mathrm{~s}$ and $30 \mathrm{~s}$ input period).

\subsubsection{Characterization of the intrinsic timescale}

The responses to forcing with periods of $\mathrm{T}=10 \mathrm{~s}$ and $\mathrm{T}=12 \mathrm{~s}$ input appear to be out of phase with respect to the inputs (see averaged response functions in figure 3.4). In these response signals, the maximal cytosolic fluorescence intensity is the first to follow an applied stimulus. Shifting the full response signal to $\mathrm{T}=10 \mathrm{~s}$ or $12 \mathrm{~s}$ forcing, with respect to the input signal and by a certain lag (approx. $3-4 \mathrm{~s}$ ) would make the signal fit to the input, in the sense that the cytosolic fluorescence minimum is the first to follow a stimulus.

Figures 3.7 A - C show the overlay of the averaged responses to a pulse for each single cell measured. The signal is computed by taking the average of each pulse response within the pulse train (i.e. the signal embedded by two consecutive stimuli). The transient response is not included. The estimated minima and maxima of the overlayed responses are highlighted by the orange and purple circles. For an input period between $\mathrm{T}=10 \mathrm{~s}$ and $\mathrm{T}=15 \mathrm{~s}$, the succession of the minimum and the maximum with respect to the pulse interval switches. This is interesting because it implies leading/lagging behavior. The timelag is defined as the time between the application of the external stimulus and the minimum of the cytosolic 
fluorescence intensity. This is calculated by the maximum correlation of the Fourier filtered inversed cytosolic signal and an artificial sum of sinusoids, approaching the pulse train. Defining the interval as going from -T/2 to $\mathrm{T} / 2$, the relative position of the minimum with respect to the stimulus $(\mathrm{t}=0)$ can be found. For $\mathrm{T}=10 \mathrm{~s}$ and $\mathrm{T}=12 \mathrm{~s}$ a lead of -2 to $-3 \mathrm{~s}$ is observed (figure 3.7D).
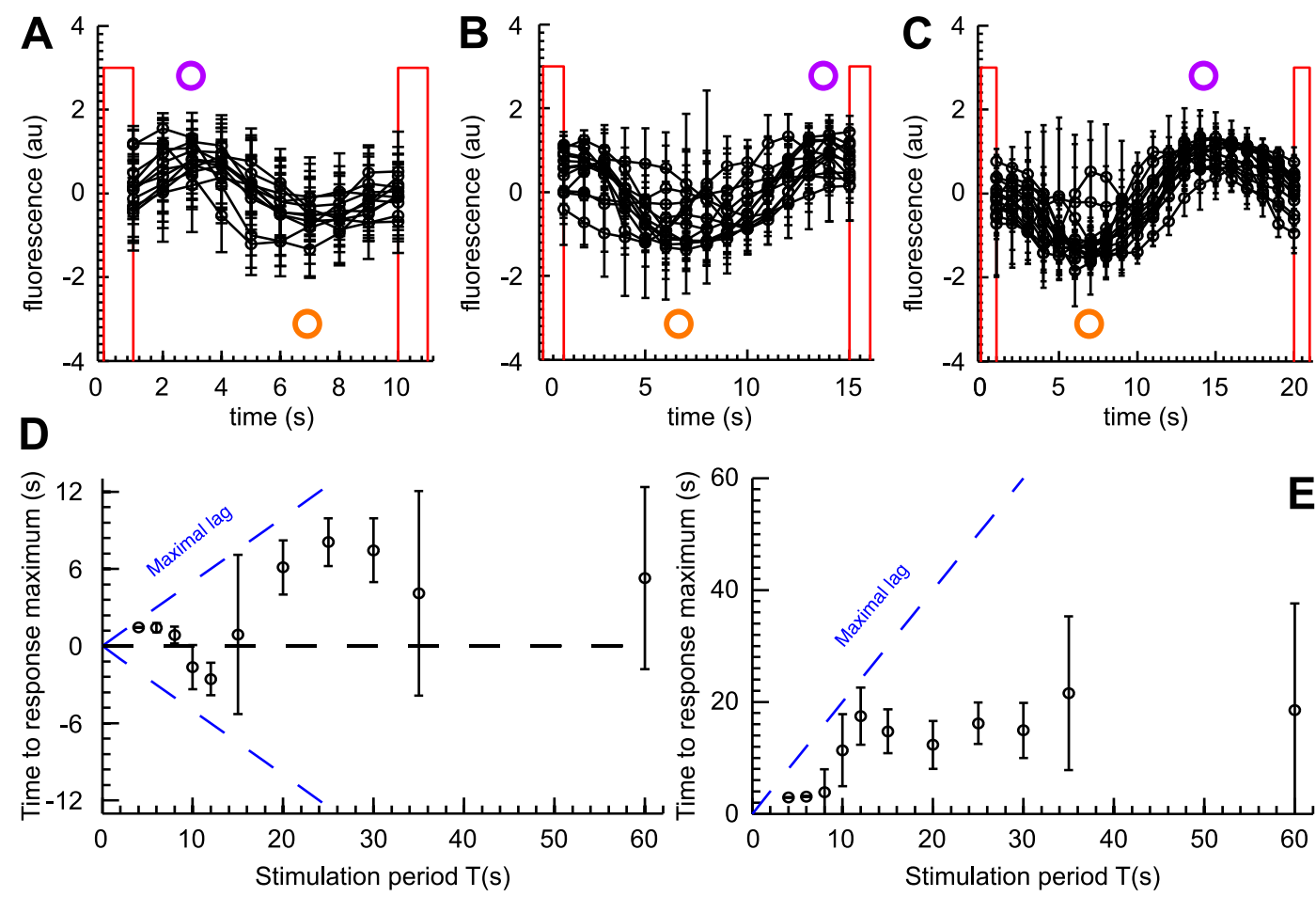

Figure 3.7.: Characterization of the intrinsic timescale. The averaged cytosolic responses of each single cell measured are overlayed for the input periods $\mathrm{T}=10 \mathrm{~s}(\mathrm{~A}), \mathrm{T}=15 \mathrm{~s}(\mathrm{~B})$ and $\mathrm{T}=20 \mathrm{~s}(\mathrm{C})$. Errorbars give the standard deviation of the mean. The transient was not included in the calculation of the averaged single cell response. Prior to averaging, the timeseries were normalized to a standard deviation of 1 . Red squares give the position of the uncaging event at time $t$ and at time $t+T$ respectively. The minimum of the cytosolic intensity shifts its position with respect to the stimulus interval as the interval increases. The approximate position of the minima and the maxima are highlighted by the orange and the purple circles respectively. (D) Response lag of the D. discoideum actin polymerization machinery and the signal processing system (i.e. the response events taking place prior to the actin polymerization). Circles give the mean of all single cell responses and their standard deviations are shown in black. The timelag between the stimulus onset and the maximal response is calculated as the lag leading to the maximum correlation between the input in output. The timelag is defined for one period and the maximal lag is indicated by the blue dashed line. For the input periods $\mathrm{T}=10 \mathrm{~s}$ and $\mathrm{T}=12 \mathrm{~s}$ the response signal seems to be out of phase. The intrinsic timescale (the time it takes to reach the fluorescence minimum) however is conserved for input periods $\mathrm{T}>8 \mathrm{~s}$. This can be seen in $(\mathrm{E})$ where the absolute value of the time lag is plotted. 
The negative time lag to the response maximum indicates that the fluorescence minimum appears right before the subsequent stimulus is applied (compare for instance in figure 3.7A the position of the minimum, marked by the orange circle, with respect to the stimulus). Note that the standard deviation of the calculated time lag derives from the single cell experiments. If the fluorescence minimum occurs at or close to $\pm \mathrm{T} / 2$ the standard deviation is large (as observed for $\mathrm{T}=15 \mathrm{~s}$ ). This is due to the single experiment detection, which is either at $+7 \mathrm{~s}$ or $-7 \mathrm{~s}$ from the stimulus, while the average gives a lag of $\approx 0 \mathrm{~s}$.

Setting the interval boundaries (i.e. the two consecutive stimuli) to 0 and $\mathrm{T}$, allows the absolute timescale of the responses to be calculated (figure 3.7F). The timescale is conserved for $\mathrm{T}=10 \mathrm{~s}$ and $\mathrm{T}=12 \mathrm{~s}$ forcing, i.e. at a value of $6-8 \mathrm{~s}$ between the applied stimulus and the subsequent occurrence of the first minimum. Therefore for $\mathrm{T}=10 \mathrm{~s}$ and $\mathrm{T}=12 \mathrm{~s}$, the cell is still responding to the first stimulus when it receives the next. We can think of the processing under this fast input as an assembly-line. The cell processes one pulse over its intrinsic timescale. A second pulse then hits the cell and passes through the signal processing system. The signal processing cascade "stores" this pulse for long enough that the cell is able to distinguish the two consecutive inputs. Shorter pulse periods, which occur faster than the processing timescale appear to the cell as a continuous input. For $\mathrm{T}=10 \mathrm{~s}$ and $\mathrm{T}=12 \mathrm{~s}$ the response signal seems to be leading and phase shifted, but when the $3 \mathrm{~s}$ signal processing time delay is included the intrinsic timescale is conserved.

\subsection{Nonlinear regimes in the actin cytoskeleton}

To investigate whether the actin system (and the signal processing) act in the linear regime, it is straightforward to apply linear response theory (see introductory section 1.5). The conditions causality (1) and stationarity (3) for linear time invariant systems can be assumed to be also fulfilled for $D$. discoideum. In fact we have shown condition (3) by measuring the response to a $\mathrm{T}=60 \mathrm{~s}$ input function. The actin cytoskeleton recovers within this time and the responses to the consecutive stimuli equal each other. Therefore, the response function is time invariant or stationary for the duration of an experiment $(\mathrm{t}<6 \mathrm{~min})$. A dose dependence (condition (2)) of the Dictyostelium filamentous actin response is known ${ }^{14}$ (Hall et al., 1988), but we would still like to discuss the applicability of linear response theory in more detail.

The transportation of cAMP over the typical length of a cell is about $0.1 \mathrm{~s}$. The concentration switch is on the order of $0.6 \mathrm{~s}$ (see material and methods section 2.3). Therefore, both

$\overline{{ }^{14} \text { Hall et al. (1988) applied step inputs with }}$ different cAMP concentrations. 
the concentration switch and the transportation of cAMP are faster than diffusion over the length of the cell. The full cell is therefore activated: (1) as a whole object (because the advection of the cAMP beats the intracellular diffusion timescale) and (2) with a certain concentration (the maximal concentration is reached very fast). This makes the approximation of a $\delta$ input reasonable on a first sight. However, the convolution of the pulse response with different input functions (created by FEM simulations) led to unsatisfactory results. Contrary to the pulse response of $E$. coli, the integral over the $D$. discoideum response is negative and therefore each convolution of a spike train with this pulse response sums up the non-zero average of the pulse response. The system pulse response as measured here by the $1.5 \mathrm{~s}$ lasting pulse can only serve as an approximation to the true pulse response. The only way to go beyond the limitations of (microfluidic) concentration switching is to deconvolve the pulse response with the pulse input. This introduces or enhances errors in the signal and has been omitted here.

In the framework of differential equations, the response to a $1.5 \mathrm{~s}$ lasting input is also a solution of this system (see introductory section 1.5). Assuming the system is linear, then a superposition of this solution is itself a solution. In figure 3.8 the comparison of superposed signals with the respective measured cytosolic responses is given. Both the measured response signal and the superposed signal were rescaled with respect to its largest absolute value. Solving a simple kicked oscillator model and applying convolution or superposition with the pulse response to recover arbitrary responses, does not require rescaling. However, in the actual experiments the input amplitude is not known precisely and furthermore, can not be compared directly to the output amplitude. Amplitude scaling is therefore necessary. Within the input period range $\mathrm{T}=4-8 \mathrm{~s}$ the superposition recovers certain features of the measured signal. For instance it revealed that for $\mathrm{T}=4 \mathrm{~s}$, as well as for $\mathrm{T}=6 \mathrm{~s}$ (data not shown) also a transient behavior is observed before reaching the steady state (see figure $3.8 \mathrm{~B}$ ). However, the steady state response of the measured signal is not oscillatory and furthermore its baseline almost recovers the prestimulation average value. The superposition fails in reproducing this behavior and therefore a nonlinear mechanism, most probably adaptation, is necessary to drive the system back to the prestimulation state. The superposed and the measured response signal for $\mathrm{T}=8 \mathrm{~s}$ are out of phase with each other (figure 3.8C). The system can not respond at the timescale given by the input function. The measured signal is therefore delayed with respect to the superposed solution. Response signals to $\mathrm{T}>8 \mathrm{~s}$ are recovered to a respectable degree by the superposition of the pulse responses (see figures $3.8 \mathrm{D}-\mathrm{G})$. We can therefore state that the oscillatory dynamics of the actin cytoskeleton and/or the signal processing system ahead of it, are dominated by a linear regime, while non-linear processes are necessary to respond to fast external forcing or a step input. 

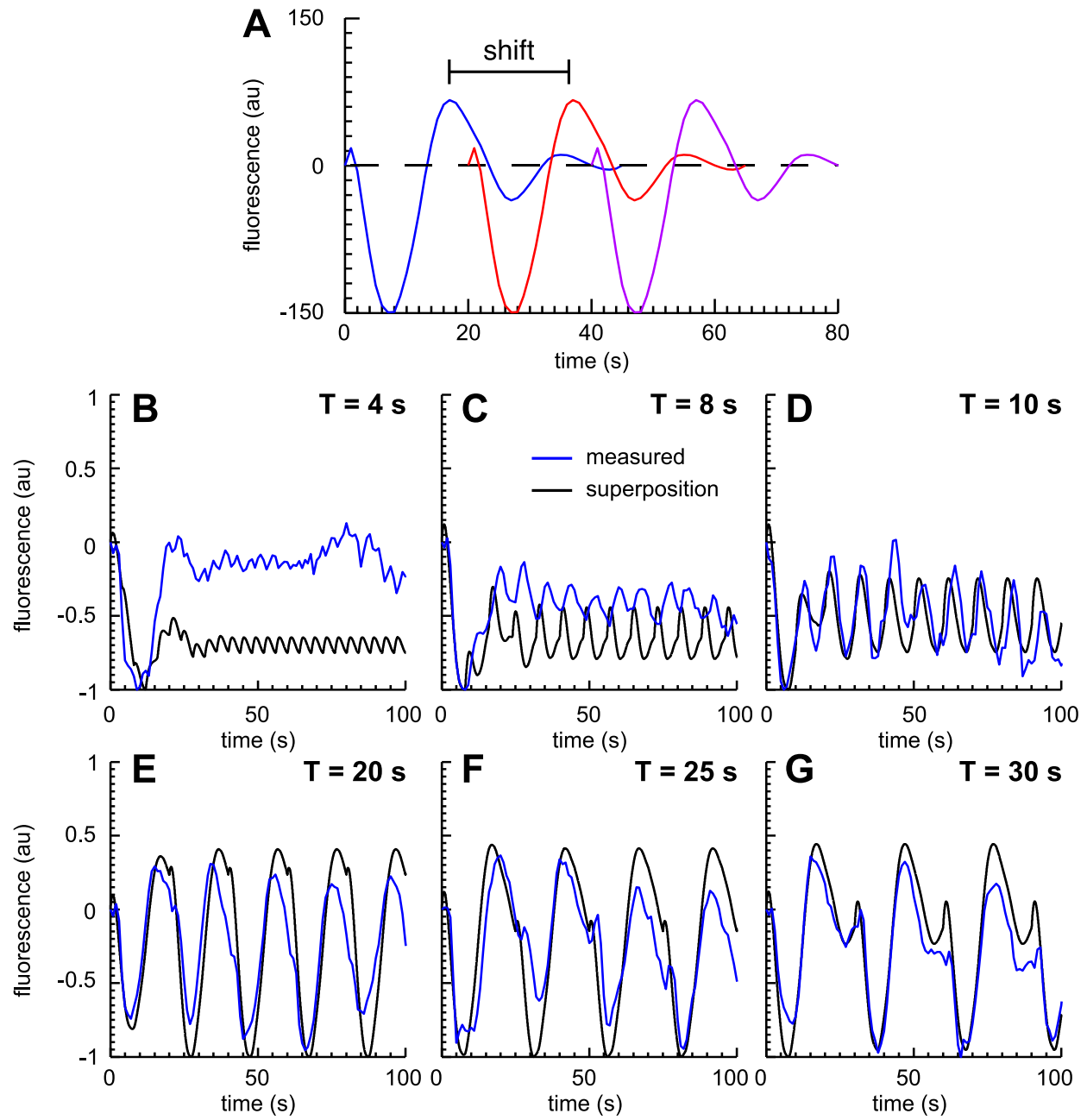

Figure 3.8.: Superposition of the pulse responses. If the system is in a linear regime, the superposition of the pulse responses should be similar to the measured response. (A) visualizes the principle of the superposition. The pulse response (blue) is overlayed with shifted pulse responses (red, purple). The length of the shift defines the desired period and finally all responses are added up. The results for different periods are displayed in $(B)$ to $(G)$. The respective measured signal is drawn in blue, while the superposition of the pulse responses is given in black. For both signals, the maximal absolute amplitude was normalized to 1 . The first uncaging event occurs at $t=0 \mathrm{~s}$. The input pulse train is not drawn for visibility. The superposition recovers particular features for $\mathrm{T}=4 \mathrm{~s}$ to $\mathrm{T}=8 \mathrm{~s}$, but nonlinear effects are necessary to explain the difference, e.g. the return of the response to $\mathrm{T}=4 \mathrm{~s}$. The response for $\mathrm{T}=10 \mathrm{~s}$ external forcing on the other hand already overlays its superposition estimate. This property holds for the longer input periods as well (G-E). 


\subsection{Theoretical approach}

In the previous sections of this chapter the intrinsic timescale of the actin cytoskeleton and the signal processing system were presented. To draw final conclusions a theoretical description of the obtained experimental results is necessary. This description should explain the intrinsic timescale, which leads to the particular resonance curve, as well as the different oscillatory patterns.

\subsubsection{Properties of the self-sustained oscillations}

Within the forcing experiments carried out, a minor fraction of autonomous oscillating cells were subject to external forcing. Two examples of $D$. discoideum cells showing an autonomous oscillation of the LimE-GFP fluorescence were exposed to periodic stimulation (figure 3.9). The cytosolic fluorescence intensity of the first cell (A) oscillates with a period of approximately $12 \mathrm{~s}$ and is exposed to an external forcing period of $\mathrm{T}=25 \mathrm{~s}$. The autooscillatory signal vanishes immediately on receiving the first pulse and is replaced by the forced pattern. Interestingly, this system returns to its prestimulus oscillatory cycle after abolishing external stimulation, indicating an underlying limit cycle. The second example (figure 3.9B) is forced with a period close to the actual period of the autonomous oscillation $(\approx 12 \mathrm{~s})$. The first stimulus is applied in phase with the oscillation, i.e. the maximum of the cytosolic fluorescence and the pulse coincidate. The cell is observed to respond at a higher amplitude to the external forcing as compared to the prestimulation amplitude. The phases of the signals over time are given in figures 3.9C and D. However, a closer investigation of the phase response requires a smaller perturbation amplitude ${ }^{15}$.

The unforced free running oscillations show an interesting amplitude-frequency relationship (figure 3.9E). The amplitudes of the self-sustained oscillations seem to decrease with decreasing frequency. The observed self-sustained oscillations of the cytosolic fluorescence intensity have an oscillation period range of 11 and $17 \mathrm{~s}$. Combining these observations with the occurrence of the different temporal response patterns to the pulse input (see section 3.1) led us to hypothesize, that the actin cytoskeletal machinery works close to the onset of an oscillatory instability. The different oscillatory patterns herein represent a different degree of vicinity to the oscillatory instability, i.e. cells, which respond with a strongly damped excursion to a pulse are considered to be far away from the instability, while the weakly damped responding cells are close but still before the instability. Finally, self-sustained oscillating cells can be seen as being beyond the oscillatory instability.

\footnotetext{
${ }^{15}$ In future experiments, the perturbation should only slightly change the amplitude of the oscillation.
} 

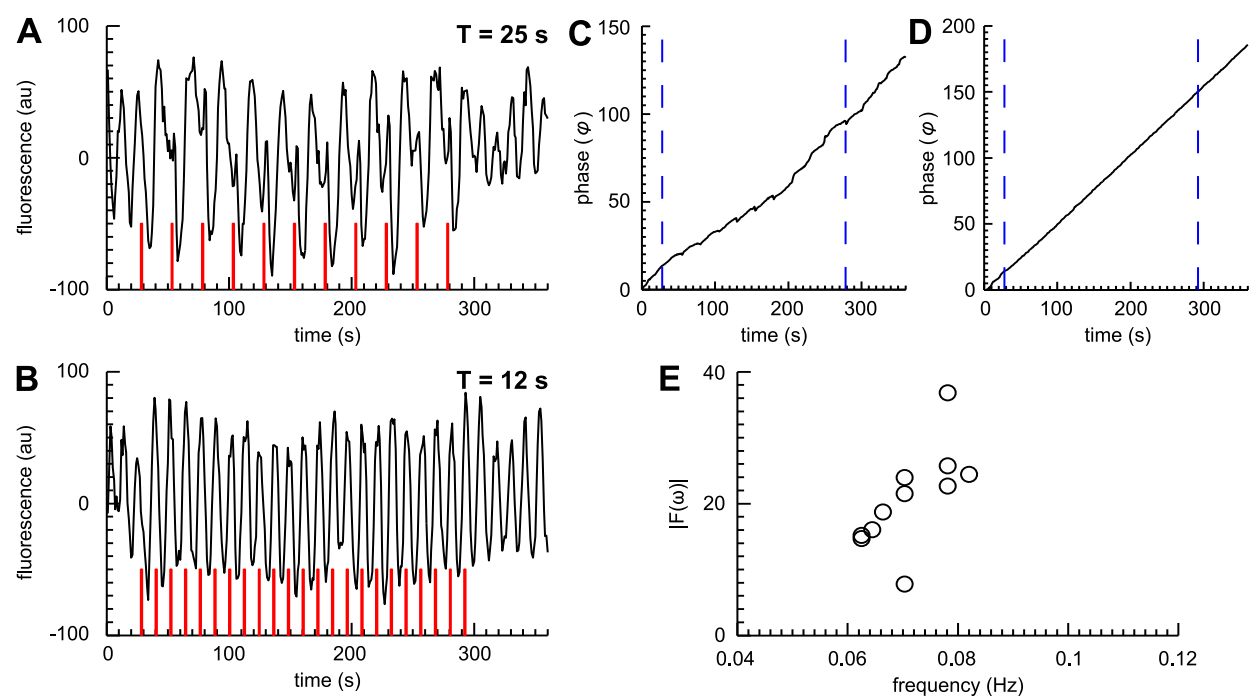

Figure 3.9.: Properties of the self sustained oscillations. (A-B) Autonomous oscillating cells exposed to periodic forcing of (A) $\mathrm{T}=25 \mathrm{~s}$ and (B) $\mathrm{T}=12 \mathrm{~s}$. In both cases the external forcing dictates the LimE-GFP fluorescence response profile. The prestimulation oscillatory pattern vanishes immediately after receiving the first external stimulus, but eventually recovers after end of external stimulation. (C) and (D) show respectively the phases of the signals in (A) and (B) over time. The blue dashed lines mark the start and the end of the periodic stimulation. A relationship between the amplitude and frequency was observed for the set of free running oscillations and is displayed in (E). The magnitude appears to decrease with decreasing frequency.

\subsubsection{Delay differential equation approach}

A simple theoretical approach, which shows the required oscillatory instability are time delay differential equations (DDE), as given by:

$$
\frac{d N}{d t}=f(N(t), N(t-\tau))
$$

where the evolution of the quantity $N$ over time is a function of the current state of the system $(N(t))$ and of a specific previous state of the system $(N(t-\tau))$ (Murray, 2003). $\tau$ gives the time delay between the current and the previous state. Here this concept has been adapted to simulate the observed temporal profiles of the filamentous actin system using the following equation:

$$
\frac{d A}{d t}=k_{+}(t) A(t)-k_{-} A(t) A(t-\tau) .
$$


$A(t)$ gives the actin concentration and its time evolution can be described straightforward by a growth $\left(k_{+}(t) A(t)\right)$ and a decay term $\left(k_{-} A(t) A(t-\tau)\right) . k_{+}(t)$ combines all polymerization enhancing factors into an effective polymerization rate. We can think of the enhancing effects as the action of the actin nucleation factors like mDia or the Arp2/3-complex. Polymerization as well as filament branching can only occur on existing filaments and therefore the growth term is dependent on the actual concentration of actin $A(t)$. The system properties are probed by perturbing $k_{+}(t)$, which is therefore necessarily a function of time. The effective depolymerization rate $k_{-}$now accounts for all polymerization inhibiting and depolymerization enhancing processes, like for instance the binding of Coronin or the action of the ADF/Cofilin complex. Since no depolymerization can occur in the absence of actin the decay term needs to be 0 for $A(t)=0$. However, it is reasonable to assume that the polymerization inhibition and the depolymerization occur with a certain delay $\tau$. Hence the magnitude of the decay term is also dependent on the actin concentration in the past $(A(t-\tau))$. For simplicity we assume that $k_{-}=k_{+}^{0}$, with $k_{+}^{0}$ giving the basal level of the effective polymerization rate. Now the fixed point of the system is found at:

$$
A^{*}=\frac{k_{+}^{0}}{k_{-}}=1
$$

The solution to the system becomes unstable with the condition:

$$
k_{+} \tau>\frac{\pi}{2}
$$

Throughout the simulations, $\tau$ was set to $\pi / 2$, so that the unstable region is designated to parameter values of $k_{+}^{0}>1$. However, the actual realization of $\tau$ in the cell is not necessarily equal to $\pi / 2$. As previously stated, the polymerization inhibiting and depolymerization enhancing factors respond with a certain time delay. To measure this delay, GFP-labeled mutants of the proteins Coronin (Gerisch et al., 1995) and Aip1 (Konzok et al., 1999) were exposed to periodic stimulation with $\mathrm{T}=20 \mathrm{~s}$. The obtained response signals are compared to the $20 \mathrm{~s}$ LimE-GFP response signal in figure 3.10A and B. As can be seen from the Fourier analysis (C-D), the depolymerization markers respond with the same frequency. However they seem to be phase shifted with respect to the LimE-GFP signal.

To quantify this time difference the steady state oscillatory parts of each signal were subject to Fourier filtering (filtered signals are displayed in figure 3.10E). The crosscorrelation was calculated for both signals folding it with the filtered LimE-GFP signal. The timelag at the maximal correlation gives an estimate of the time delay between the polymerization and the depolymerization maxima. It yields $\tau=4.3 \mathrm{~s}$ for the Aip1-GFP and $\tau=5.6 \mathrm{~s}$ for the 
Coronin-GFP signal. Therefore, the value for the actual realization of $\tau$ was set to $5 \mathrm{~s}$. This simple model is already able to recover the different observed oscillatory states (as summarized in figure 3.2).
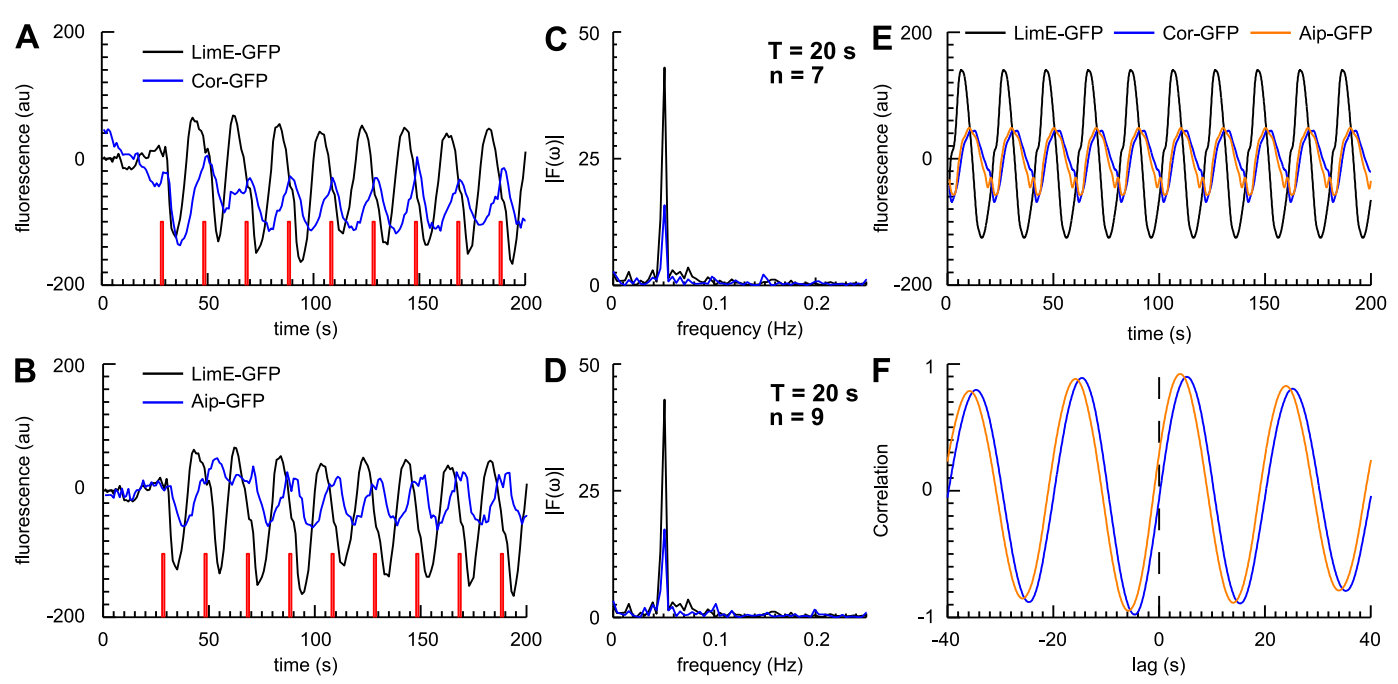

Figure 3.10.: Characterization of the time delay between the polymerization and the net depolymerization of filamentous actin. $D$. discoideum cells expressing the depolymerization markers CoroninGFP (A, blue) and Aip1-GFP (B, blue) were exposed to $\mathrm{T}=20 \mathrm{~s}$ periodic stimulation (red). The respective response signals are compared to the LimE-GFP response for $\mathrm{T}=20 \mathrm{~s}$ (black). (C) and (D) show the comparison of the Coronin-GFP and the Aip1 response in the frequency domain. (E) overlays the Fourier-filtered response signals. The FFT filter was exclusively applied to the steady state part of the response signal. The crosscorrelation of these filtered Coronin and Aip1-GFP response signals is shown in $(\mathrm{F})$. The timelag to the maximal correlation gives the delay time between the polymerization and the depolymerization of the filamentous actin.

However, the process of adaptation is necessary to explain the response to a continuous input (see figure 3.1B). The cytosolic fluorescence intensity recovers to its prestimulation value, indicating the relocalization of LimE-GFP to the cytosol. A non-adapting system would oscillate, contrary to this, around a new base value. We implemented adaptation into the definition of $k_{+}(t)$ :

$$
k_{+}(t)=k_{+}^{0}+(a(t)-b(t)) .
$$

The new variable $a(t)$ simulates the input. The pulse input is defined as a gaussian distribution, with the amplitude $\varepsilon$ and the variance $\sigma$. Consequently the pulse trains are simulated as consecutive series of pulses. The step input is approached by the error function, which also is multiplied by $\varepsilon$ to weight the amplitude. 
Finally, $b(t)$ gives the adaptation within the system to a certain input $a(t)$. The time evolution of $b(t)$ reads:

$$
\frac{d b}{d t}=\frac{1}{\tau_{a d}}(a(t)-b(t))
$$

The second timescale $\tau_{a d}$ can be regarded as the adaptation timescale. It defines how fast the adapting variable $b(t)$ reaches the value of $a(t)$ and was determined from the response to a continuous stimulation. An exponentially damped cosine was fitted to the cytosolic response (see figure 3.1B for the step response). Mean, amplitude, frequency and phase of the cosine were fitting parameters and could be well estimated from the frequency domain. Finally, the fitted decay timescale $\tau_{a d}=12.5 \mathrm{~s}$ serves as an estimate of the adaptation timescale and was used throughout the simulation.

\subsubsection{Evaluation of the model}

For the evaluation of the model, parameters $\varepsilon$ and $\sigma$ were chosen to be 0.5 and 0.3 , respectively. The results of the pulse and the continuous perturbation are displayed in figure 3.11. Strongly damped responses to a pulse input can be found in the $k_{+}^{0} \ll 1$ regime (A), while the weakly damped responses are represented by $k_{+}^{0}$ close to, but still smaller, than 1 (B). Finally the self-sustained oscillations are recovered for $k_{+}^{0}>1(\mathrm{C})$. Note that all simulations were carried out in units of $\tau$, but were converted into time units for better comparability ${ }^{16}$. The pattern of the step response shows a similar profile. However, as also observed experimentally, the timescales of the step response are longer than the pulse response timescales. This is due to the effective input (i.e. the timescale within $k_{+}(t)>k_{+}^{0}$ holds), which itself is longer than the pulse input. Furthermore the amplitude of the depletion is larger in the step response, which has not been observed experimentally. This can be explained, since the cytosolic fluorescence has a lower limit (entire depletion of the cytosol from LimE-GFP), below it cannot drop.

Figure 3.12 shows the simulations of the actin concentration under different periodic perturbations. Again the experimentally observed temporal patterns were recovered qualitatively by the model. Particularly striking is the recovery of the transient behavior of the response for $\mathrm{T}=6 \mathrm{~s}$ (compare the experimentally obtained (figure 3.3D) and the simulated timeseries (figure $3.12 \mathrm{~A}$ ) in the time range $40-60 \mathrm{~s}$ ). The response profiles for increasing period lengths show an amplitude increase towards the input period of $\mathrm{T}=20 \mathrm{~s}$ (compare figures 3.12B and C) and finally also the second smaller response occurs for input periods larger than $\mathrm{T}=30 \mathrm{~s}$. All simulations were carried out with a $k_{+}^{0}=0.7$, because on average the

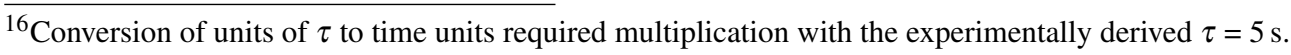


pulse response is strongly damped and the fraction of weakly damped single cell examples is smaller than $10 \%$. The resonance curves for the different $k_{+}^{0}$ were calculated as for the experimentally obtained time series and are plotted for $k_{+}^{0}=0.6$ and 0.7 (figures 3.12E and F). The amplitude response shows a peak at an input period of $\mathrm{T} \approx 23 \mathrm{~s}$, which is in agreement with the experimental observation of a resonance at $\mathrm{T}=20 \mathrm{~s}$ periodic stimulation. Furthermore, a second resonance peak becomes apparent for $k_{+}^{0} \geq 0.7$, which can also be found in the experimentally obtained amplitude response (see figure 3.7C). Summarizing, one can conclude that the simple DDE model approach already recovers the main qualitative features of the actin cytoskeletal system under external stimulation. However, the DDE model fails to reproduce the baseline shifting (see figure $3.4 \mathrm{~K}$ ) and also creates an overestimate of the overshoot, in both, amplitude and length.
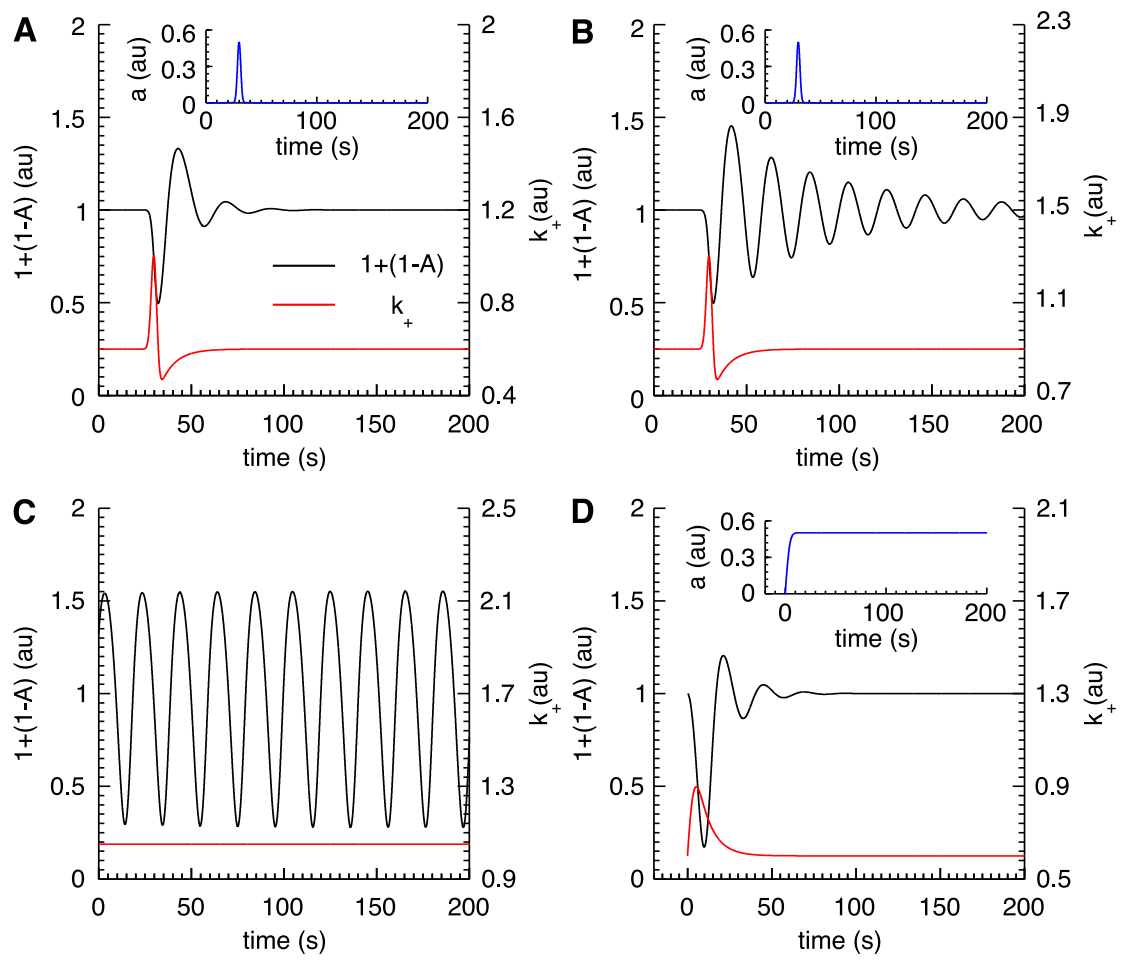

Figure 3.11.: Simulation of the pulse and the step response of the DDE model. The input (i.e. $a(t)$ ) is given in the respective subplots. The response (i.e. the actin concentration $A(t)$ ) is always plotted as $1+(1-\mathrm{A})$ to be easier compared with the cytosolic fluorescence profiles obtained in the experiments (black line). The effective polymerization rate over time $\left(k_{+}(t)\right)$ is drawn in red. (A-C) give the three observed oscillatory states: (A) strong damping $\left(k_{+}^{0}=0.6\right)$, (B) weak damping $\left(k_{+}^{0}=0.9\right)$ and (C) free running oscillations $\left(k_{+}^{0}=1.05\right), k_{+}(t)$ adapts, if the input $a(t)$ is continuous. The damping of the response to a step input is also dependent on $k_{+}^{0}$ and in (D) shown for the case $k_{+}^{0}=0.7$. The input simulated as the errorfunction was only defined for $t \geq 0 \mathrm{~s}$ (see subplot in (D)) 

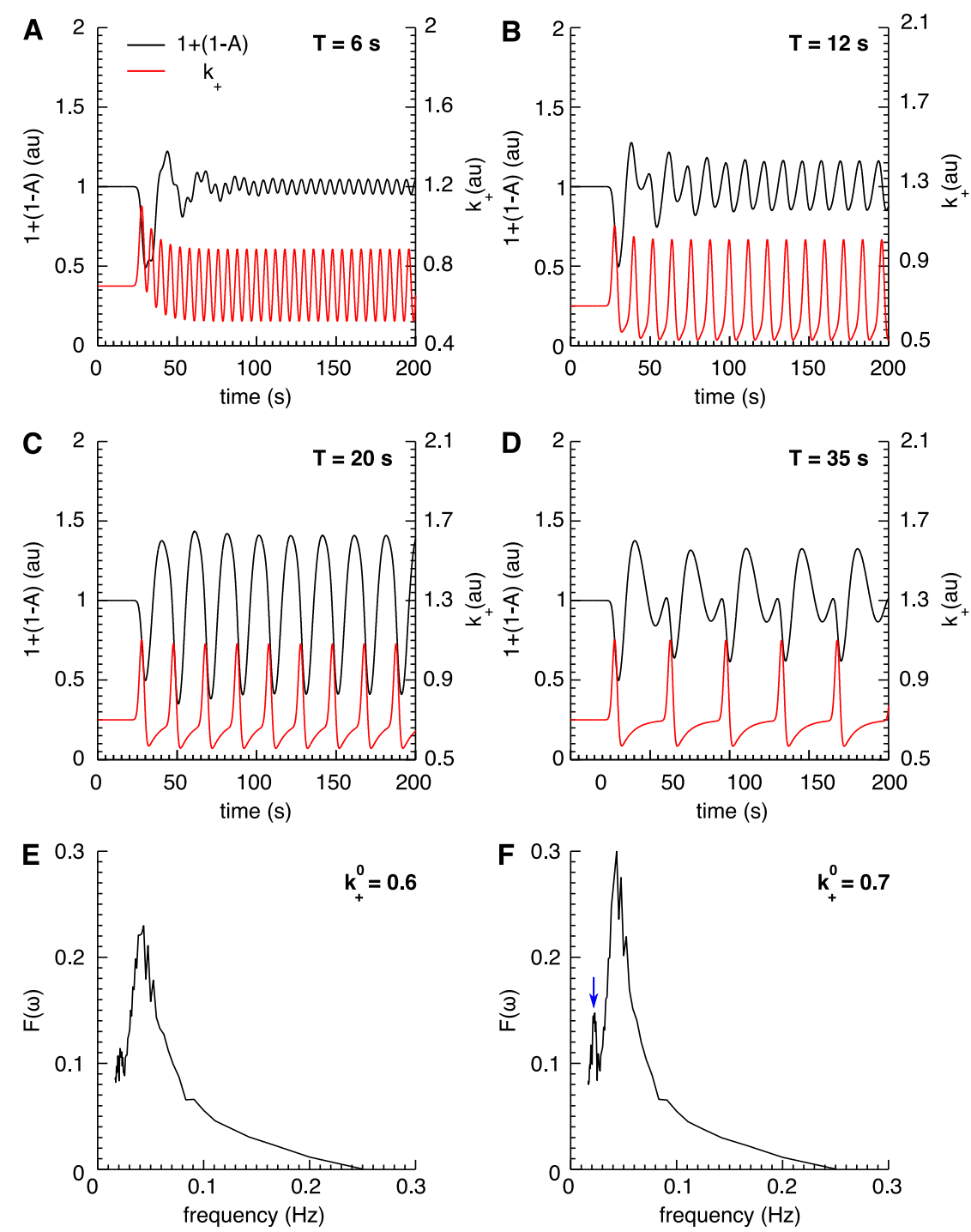

Figure 3.12.: Response of the delay differential equation model to periodic pertubation. (A-D) Simulation of the actin concentration over time, if the system is exposed to different pertubation periods. These can be qualitatively compared with the obtained response signals in the experimental section. Again $A(t)$ is plotted in black as $1+(1-\mathrm{A})$ for convenience and $k_{+}$is drawn in red. $k_{+}^{0}$ was equal to 0.7 in all simulations. Periods between $\mathrm{T}=4 \mathrm{~s}$ and $\mathrm{T}=60 \mathrm{~s}$ periodic pertubation were simulated and the amplitude response was calculated as for the experimentally obtained timeseries. (E) $k_{+}^{0}=0.6$ and (F) $k_{+}^{0}=0.7$. In both cases the resonance can be found at approximately $1 / 23 \mathrm{~Hz}$. The second smaller peak (indicated by the blue arrow) is only visible for $k_{+}^{0} \geq 0.7$. 


\subsection{Discussion}

The here described onset of an oscillatory response at input periods $\mathrm{T}>8 \mathrm{~s}$ is considerably shorter, than the recently estimated timescale by Wang et al. (2012). This group periodically stimulated $D$. discoideum cells with cAMP pulse periods down to $\mathrm{T}=17 \mathrm{~s}$ and concluded from the CRAC-GFP ${ }^{17}$ response, that $D$. discoideum cells cannot time resolve periodic inputs lower than $\mathrm{T}=20 \mathrm{~s}$. In general, one should not rely on only a single label to deduce the properties of the entire system. The CRAC-GFP label for instance, responds as fast as the LimE protein (translocation becomes apparent about $3 \mathrm{~s}$ after the stimulus reception), but it shows a longer decay back to its a prestimulation value (Etzrodt et al., 2006). Hence the label might need longer input periods, compared to the LimE-GFP label, to build up an oscillatory response ${ }^{18}$. Indeed the response of the CRAC-GFP label to $\mathrm{T}=10 \mathrm{~s}$ external forcing shows a prolongued initial response but no oscillatory steady state response (see figure 3.13A), while the corresponding LimE-GFP signal is oscillatory (figure 3.4B). The external forcing always acts on the entire system, but the observed label only shows one single integrative part of the system. Here we state that the periodic forcing of the LimE-GFP label shows, that $D$. discoideum amoebae can temporarily resolve periodic input functions down to at least $\mathrm{T}=10 \mathrm{~s}$.

The comparison of chemotaxis associated proteins with filamentous actin markers in the low external forcing regime may provide further insight to the question, which factor actually limits the dynamics of the actin cytoskeleton, as asked by Carlsson (2010). The author concludes by simple timescale comparison, that the dynamics must be set by the timescales of the upstream signal processing system. Periodic stimulation could be used to tackle this task. As one approaches the limit of the temporal resolution, more and more labeled proteins show a step like response, leaving the last periodically responding protein as the one, which limits the timescale ${ }^{19}$.

If linear systems are subject to a continuous pertubation, which is rapidly shut off, they return to their initial fixed point. That means these systems run through the exact opposite behavior to the step response. This "step-off" or "step-out" response in fact has been recently observed in D. discoideum using the Ras-GFP label (Takeda et al., 2012), but could not be observed with the LimE-GFP label (data not shown, experiments carried out by José Negrete Jr.). Yet another indication, that the properties of the entire system (i.e. the cell) should not be deduced right away from just a single labeled protein.

\footnotetext{
${ }^{17} \mathrm{CRAC}$, the cytosolic regulator of the adenylate cyclase, is a marker for the early chemotactic response.

${ }^{18}$ Recall that, in the case of LimE-GFP we could relate the onset of the oscillatory response directly to the pulse response timescale.

${ }^{19}$ This of course assumes that the limiting factor has in fact a measurable translocation profile.
} 
The response of the actin associated molecular motor protein myosin is about $10-20 \mathrm{~s}$ delayed to the LimE-GFP response. Cells expressing MyoII-GFP tags had been exposed to medium and high external forcing. The responses to $\mathrm{T}=20 \mathrm{~s}$ and $\mathrm{T}=30 \mathrm{~s}$ external $\mathrm{cAMP}$ stimulation are given in figures 3.13B - C. Both oscillatory timetraces show a response in a 1:1 manner, i.e. the dominant response frequency equals the input frequency (figures 3.13 $\mathrm{G}-\mathrm{H}$ ). While the actin cytoskeletal response, marked by LimE-GFP, already undergoes the process of period halving, remains the Myosin response in the 1:1 regime. The Myosin response has been shown to need several input pulses to build up a measurable response (Ishikawa-Ankerhold, 2008). For $\mathrm{T}=20 \mathrm{~s}$ this is in agreement (it takes about $4-5$ consecutive stimuli to result in a measurable Myosin translocation), while the $30 \mathrm{~s}$ response is not, making a larger set of samples necessary.

This work also draws some suggestions for further experimental work using the flow photolysis method. A critical step was the characterization of the DMNB-caged cAMP by HPLC and indirectly via DMNB-caged fluorescein. In the early 2000's the quantification of the released amount of uncaged material was improved by introducing the 6,7biscarboxymethoxy-coumarin-4ylmethyl (BCMCM), as well as the 7-diethylamino-coumarin-4-ylmethyl (DEACM) ester (Hagen et al. (2001), Hagen et al. (2002)). Both caging groups are fluorescent upon release from the caged precursor and would simplify the characterization of the released material. The DEACM-cage shows better uncaging efficiency in the near UV and violet range, making it easier accessible via the $405 \mathrm{~nm}$ laser. The BCMCM cage improves the solvability in water and does not require to be solved in DMSO prior to dilution. Further studies should thus switch to the now commercially available BCMCMor DEACM-caged cAMP variants.

Interestingly, the determination of the time delay between the LimE-GFP and the Coronin/ Aip1-GFP fluorescence peak via correlation of the periodic forced response signals (figure 3.10) opened a new possibility to measure the timescales of the system. It compares the time delay in the steady state regime. Due to the correlation over the entire length of the signals, it is a more accurate method to estimate the delay between two different labels, than the response to a sudden upshift of cAMP.

Other labels that mark the filamentous actin system tag the actin binding domain (ABD) (Pang et al., 1998) or more recently "lifeact" actually only associates not more than a small actin binding peptide to the GFP (Riedl et al., 2008). Its small size supposably limits artificial effects, but due to its recent development ${ }^{20}$ it had not been applied within this work. Actin-GFP, changes the polymerization kinetics, but is contrary to LimE-GFP a direct staining. Vicker and Grutsch (2008) for instance used Actin-GFP to determine the timescale of

${ }^{20}$ The first application of GFP-lifeact in D. discoideum can be found in Neilson et al. (2011) 
passing cAMP waves in streaming D. discoideum cells. Here, Actin-GFP labeled cells were forced with $\mathrm{T}=10 \mathrm{~s}$ external stimulation period. Results are given in figure 3.13D and are comparable to the LimE-GFP results (see frequency spectrum in figure 3.13I).

Next to the pulse and the step input, a sinusoidal input is often applied to characterize the system properties (see for instance Tu et al. (2008)). It serves to evaluate the frequency response of a system, but was not applied in this study. The realization of a sine wave using laser based photolysis faces a number of experimental difficulties. However, its application in general might not be valid, since the cAMP concentration needs to be always positive (or zero) and the sine wave would consequently have a non-zero mean. The $D$. discoideum chemotactic system would unavoidably adapt to the mean of the sinusoidal input.

The delay differential equation model, introduced in the previous section, is a purely temporal approach as the obtained processed data contains no spatial dependencies. Current models of actin based motility however are spatial and include the major characteristics (like filament density, association/dissociation rates etc.) of the actin polymerization and depolymerization machinery. Oscillatory instabilities are known from these models, as they were used to explain for instance the oscillatory movement of Listeria mutants ${ }^{21}$ (Lacayo et al. (2012), Enculescu and Falcke (2011), Gholami et al. (2008)). Oscillatory instabilities have further been reported in other cytsokeletal structures. The molecular motors are known to spontaneously create collective oscillations, by the synchronization of their detachment and reattachment onto the filament (Julicher and Prost, 1997). However, it is beyond the scope of the DDE model to allow insight into the underlying physics or chemistry of the actin polymer. It provided an explanation of the observed temporal dynamics of the actin cytoskeleton in vivo in chemotactic amoebae.

Finally, damped oscillations in response to an external stimulus have been observed in human leukocytes previously (Wymann et al., 1990). These cells respond with respiratory oscillations to a sudden chemotactic stimulus. If these respiratory oscillations are artificially suppressed, transient F-actin oscillations become apparent. Shape oscillations could also be induced in polymorphonuclear leukocytes, if stimulated with its chemoattractant LTB $_{4}$ (Omann et al., 1989). Furthermore, a biphasic type of chemotactic response has been reported in leukocytes (Norgauer et al., 1994). The here drawn conclusions of an oscillatory instability in the actin cytoskeletal system, might thus not be limited to chemotactic amoebae.

\footnotetext{
${ }^{21}$ These pathogenic prokaryotic organisms propel themselves forward, by recruiting the actin cytoskeletal machinery of the infected host cell.
} 

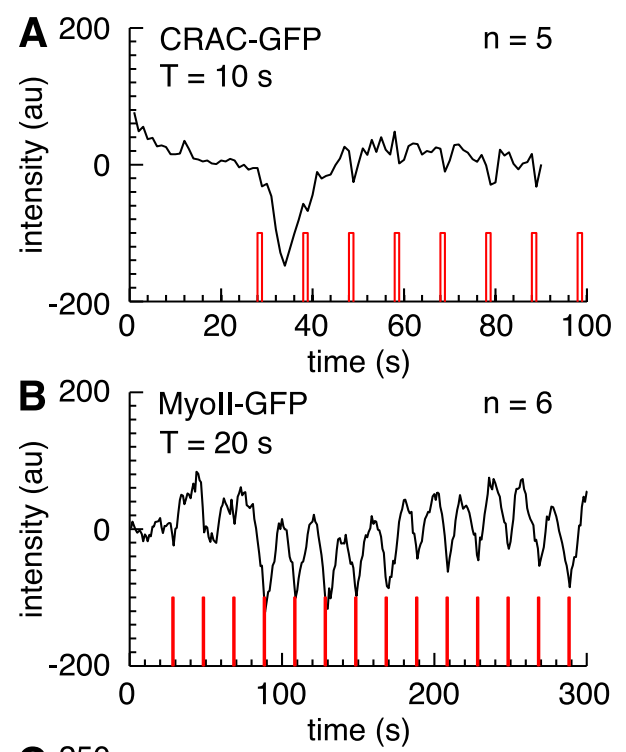

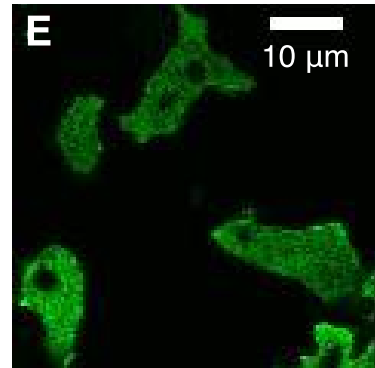

Myoll-GFP

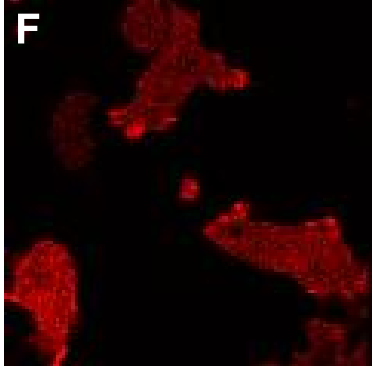

LimE-mRFP
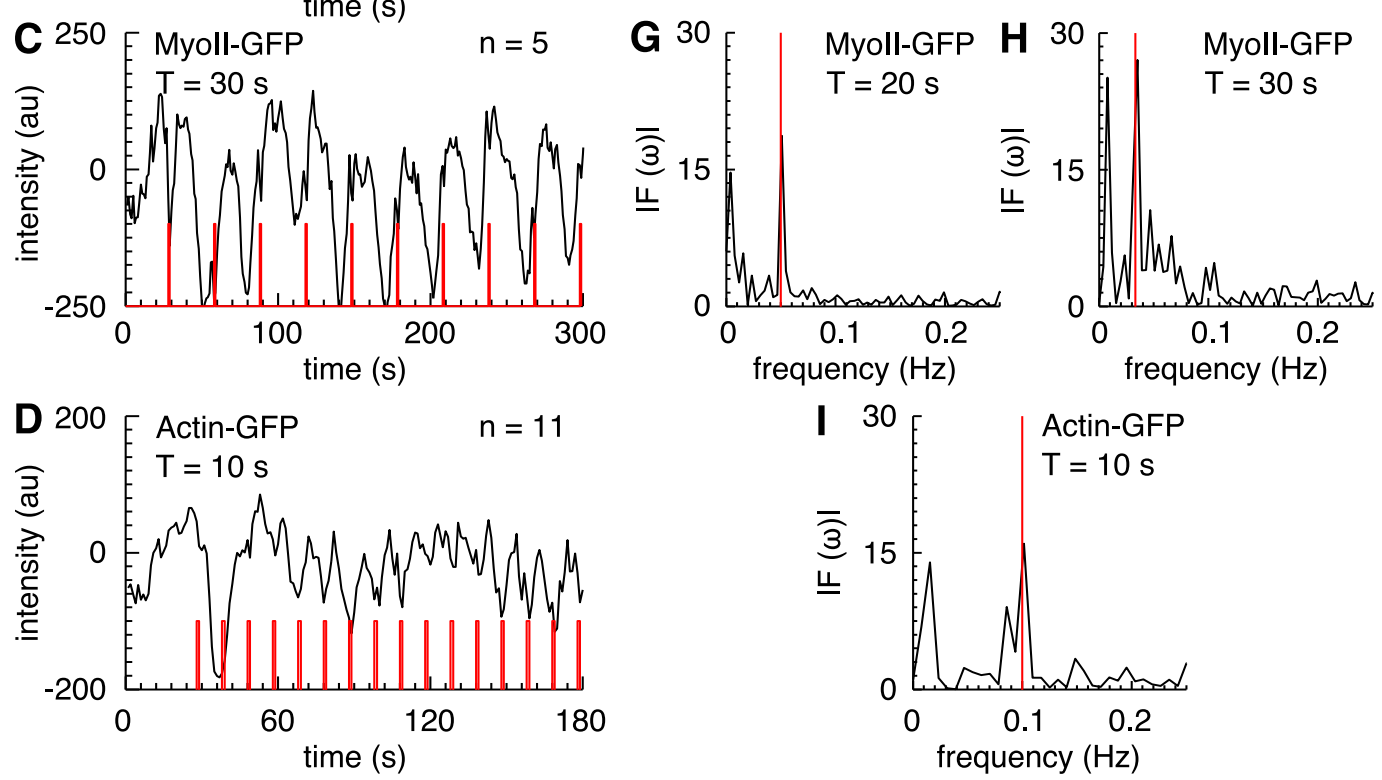

Figure 3.13.: Periodic stimulation of other markers of the chemotactic or the cytoskeletal response. (A) Cytosolic CRAC-GFP response to a $\mathrm{T}=10 \mathrm{~s}$ cAMP input. Although the corresponding LimEGFP response is oscillatory, the CRAC-GFP response resembles a step answer. The small depletions can be related to the GFP itself as in figure 3.13. (B-C) Response of the MyoII-GFP label to external driving of periods $\mathrm{T}=20 \mathrm{~s}(\mathrm{~B})$ and $\mathrm{T}=30 \mathrm{~s}(\mathrm{C})$. (D) Cytosolic fluorescence response of the direct actin label Actin-GFP for $\mathrm{T}=10 \mathrm{~s}$. (E-F) Representative images of the MyoII-GFP (green) and the LimE-mRFP label in this cell line. The MyoII-GFP label shows small zones of fluorescence exclusion. The image processing was adapted in a way, that these exclusions did not contribute to the cytosolic fluorescence average. (G-I) The frequency spectra, obtained from the steady state response of the time series in (B-D). Red line indicates the driving frequency. 


\subsection{Chemotactic response to short-time stimuli}

\subsubsection{Results}

Traditionally chemotactic experiments on $D$. discoideum are carried out exposing the cell to a stable gradient of cAMP (via a pipette, flow chamber or a microfluidic gradient mixer (Jin, 2009)). In the previous sections the periodic stimulation of the actin cytoskeleton has been described. However the $D$. discoideum cell finally responds with chemotaxis towards cAMP. Here we will use the existing data to investigate the chemotactic response of the $D$. discoideum to periodic sequences of cAMP with individual pulse lengths of $\approx 1.5 \mathrm{~s}$.

Celltracks of migrating $D$. discoideum cells were calculated as the $(\mathrm{x}, \mathrm{y})$ position of the cell centroid over time, using a customized MATLAB cell tracking algorithm written by Crocker and Gierer. A detailed description of the algorithm can be found in Theves (2009). A mean flow speed of $120 \mu \mathrm{m} / \mathrm{s}$ is necessary to achieve these short time pulses. First one has to rule out that the mechanical stress on the cell, by the applied microfluidic flow, leads to an intracellular reorganization and subsequent polarization, as has been described by Dalous et al. (2008). Figure 3.14A shows the result for D. discoideum moving in the microfluidic channel in the absence of stimulation. The cells move in a random manner, i.e. the histogram of the velocities in $\mathrm{x}$-direction $\left(v_{x}\right)$ is uniform (figure 3.14D), which indicates that the flow velocity is still low enough to place no bias on the movement. The histograms give the distribution of all individual velocities in $\mathrm{x}$-direction. Those are calculated as $\Delta x / \Delta t$ from each individual celltrack, whereas $\Delta t=3 \mathrm{~s}$.

A single $1 \mathrm{~s}$ lasting uncaging event induces no chemotactic movement towards the uncaging region in the following $120 \mathrm{~s}$, after the pulse was applied (see 3.14B and E), although the stimulus leads to a clear actin localization (see figure 3.1C) and the cringe response. However, D. discoideum cells recognize the gradient during continuous stimulation (figures $3.14 \mathrm{G})$. The histogram of the $v_{x}$ shows a clear bias towards negative $v_{x}$, i.e the cells move towards the uncaging region. We should recall that the established gradient is a concentration difference between the front and the back of the cell (while the front is the cell region closest to the uncaging region), which is introduced by the diffusive spreading of the profile. Neglecting any effects of the cell on the concentration in the flow (as described in Beta et al. (2008)), one finds the concentration difference ${ }^{22}$ to be approximatly $8 \%$. Given Dictyosteliums ability to sense gradients down to $2-5 \%$ (Parent et al., 1998) difference over the cell, the directional movement of the amoeba towards the continuously occurring source of cAMP is not surprising.

\footnotetext{
${ }^{22}$ Concentrations are calculated by finite element simulation. See the material and methods section 2.3 for more details.
} 

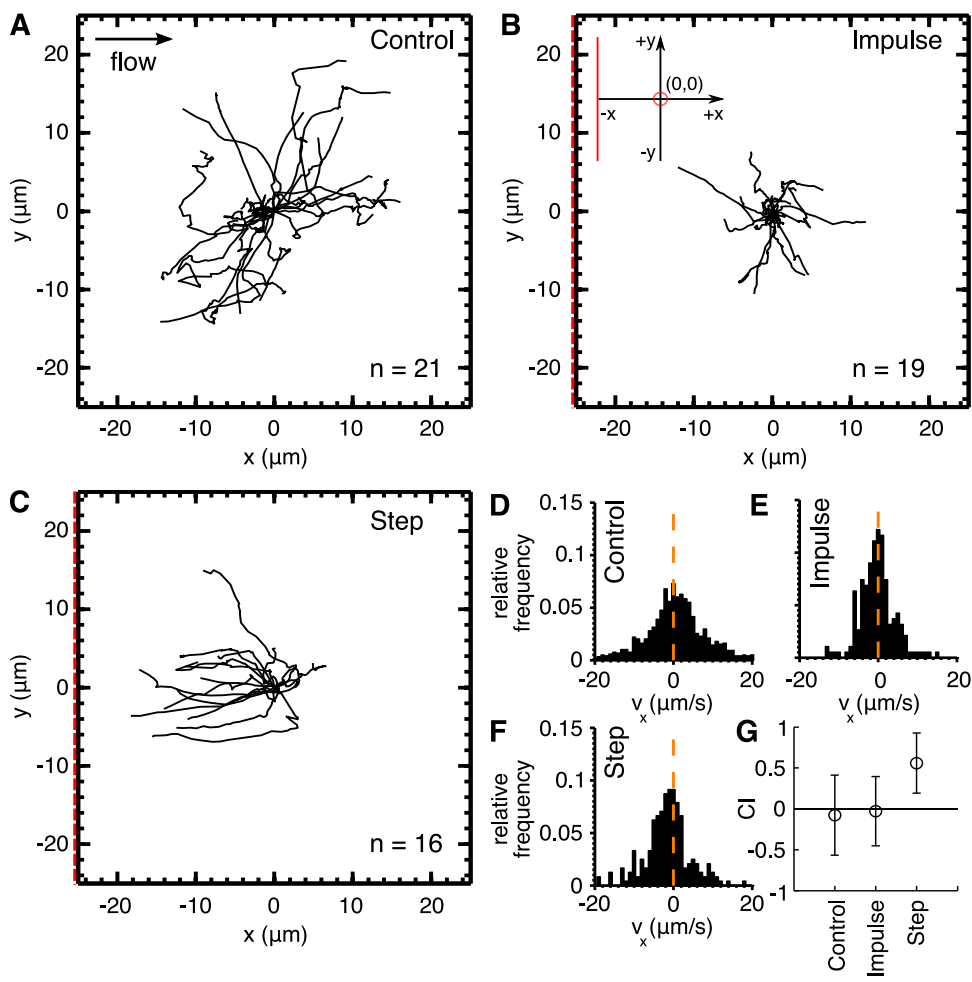

Figure 3.14.: Chemotactic response to an pulse and a step input function. (A-C) contain the cell motion monitored as the centroid position over time. The control (A) shows the motion in absence of external stimuli. The position of the uncaging region is marked with a red dashed line in (B) and $(C)$. The position of the cell centroid at the application of the initial stimulus is set to $(x, y)=$ $(0,0)^{23}$. (D-F) show the histograms of all velocities in $\mathrm{x}$ direction. A bias towards negative values proofs a chemotactic motion towards the uncaging region (see the coordinate system in B). The orange dashed line indicates $v_{x}=0 \mu \mathrm{m} / \mathrm{s}$. Chemotactic responsitivity to different input functions is measured by the chemotactic index (CI). The $\mathrm{CI}$ for the control (no external stimulation) and the $\mathrm{CI}$ for cells exposed to an impulse and a step input is given in (G).

The directionality of the control, as well as the pulse and stimulus input function are to be compared to the directionality of the periodic train responses in terms of the cell velocity in $\mathrm{x}$-direction ${ }^{24}$ and the chemotactic index. The chemotactic index (CI) is defined as:

$$
C I=v_{x} / \bar{v}
$$

\footnotetext{
${ }^{23}$ Note that the pulse response celltracks are limited arbitrarily to $120 \mathrm{~s}$ and not by the duration of the external stimuli.

${ }^{24}$ In figure 3.14B the respective coordinate system is illustrated. This holds for all celltracks in the subsequent figures.
} 
and measures the directionality of the movement (Fuller et al., 2010) ${ }^{25}$. The closer the CI is to 1 the closer is the movement to a straight line towards the uncaging region ${ }^{26}$. The histograms of the $v_{x}$ distribution are symmetric around $0 \mu \mathrm{m} / \mathrm{s}$ for the control and the single pulse response, but the control distribution shows a wider profile. This is probably due to the cringe response. In response to a sudden chemoattractant stimulation, a cell stops its current movement and the cell shape cringes. The timescale of this response is around $20 \mathrm{~s}$ (Futrelle et al., 1982). Therefore in response to a single pulse, the distribution is thinner around small absolute values of $v_{x}$. Due to its symmetric distribution, both the control and the single pulse response have an averaged chemotactic index $\approx 0$ (figure 3.14G). The step responses $\mathrm{CI}$ is on average $\approx 0.5$ and can serve here as the positive control.

A short view on the celltracks in response to periodic forcing, plotted in figure 3.15A - E, reveals already that the cells detect the uncaging source if the pulses are applied periodically. The bias towards the uncaging region is prominent under $\mathrm{T}=4$ to $\mathrm{T}=35 \mathrm{~s}$ driving, but the chemotactic efficiency is not constant for varying periods. The histograms $3.15 \mathrm{G}-\mathrm{K}$ show different strengths of biases towards negative values of $v_{x}$. Low input periods ( $\mathrm{T}=4 \mathrm{~s}$ and $\mathrm{T}=6 \mathrm{~s})$, as well as high periodic forcing $(\mathrm{T}=25 \mathrm{~s}$ to $\mathrm{T}=35 \mathrm{~s})$ impose only a small bias towards the uncaging region on the cell movement. The strongest bias is observed for $\mathrm{T}=8 \mathrm{~s}$ to $\mathrm{T}=20 \mathrm{~s}$. One recognizes the differences in the shape of the histograms, by looking at the large negative values of the velocity in $\mathrm{x}$-direction. The response for $\mathrm{T}=8 \mathrm{~s}$ input shows a higher amount of fast moving cells ${ }^{27}$, when compared to the histogram of for instance $\mathrm{T}=20 \mathrm{~s}$. On average, cells exposed to stimuli with periodicities ranging from $\mathrm{T}=8-20 \mathrm{~s}$ respond with a CI close to the $\mathrm{CI}$ of the step response. This is summarized in figure $3.15 \mathrm{H}$, where the chemotactic index plotted as a function of input period. As observed for the actin cytoskeletal response, in the limit of isolated single pulses ( $T=60 \mathrm{~s}$, figure $3.15 \mathrm{~F}$ and $\mathrm{L}$ ), the response becomes similar to the control in the sense that the histogram of the velocities in $\mathrm{x}$ direction is symmetric. However, as prominent in the response to a single pulse, for $\mathrm{T}=60 \mathrm{~s}$ the cells are stopped or slowed down periodically by the repeating cringe response.

\footnotetext{
${ }^{25}$ The CI was implemented into the MATLAB routine chemotacticindex.m by Matthias Theves.

${ }^{26}$ For simplicity the uncaging region (i.e. the source of cAMP) is reduced to a point at $(\mathrm{x}, \mathrm{y})=(-24 \mu \mathrm{m}, 0 \mu \mathrm{m})$.

${ }^{27}$ Note that the amount of faster moving cells is underestimated because these cells leave the imaging region faster and therefore contribute less to the histogram.
} 

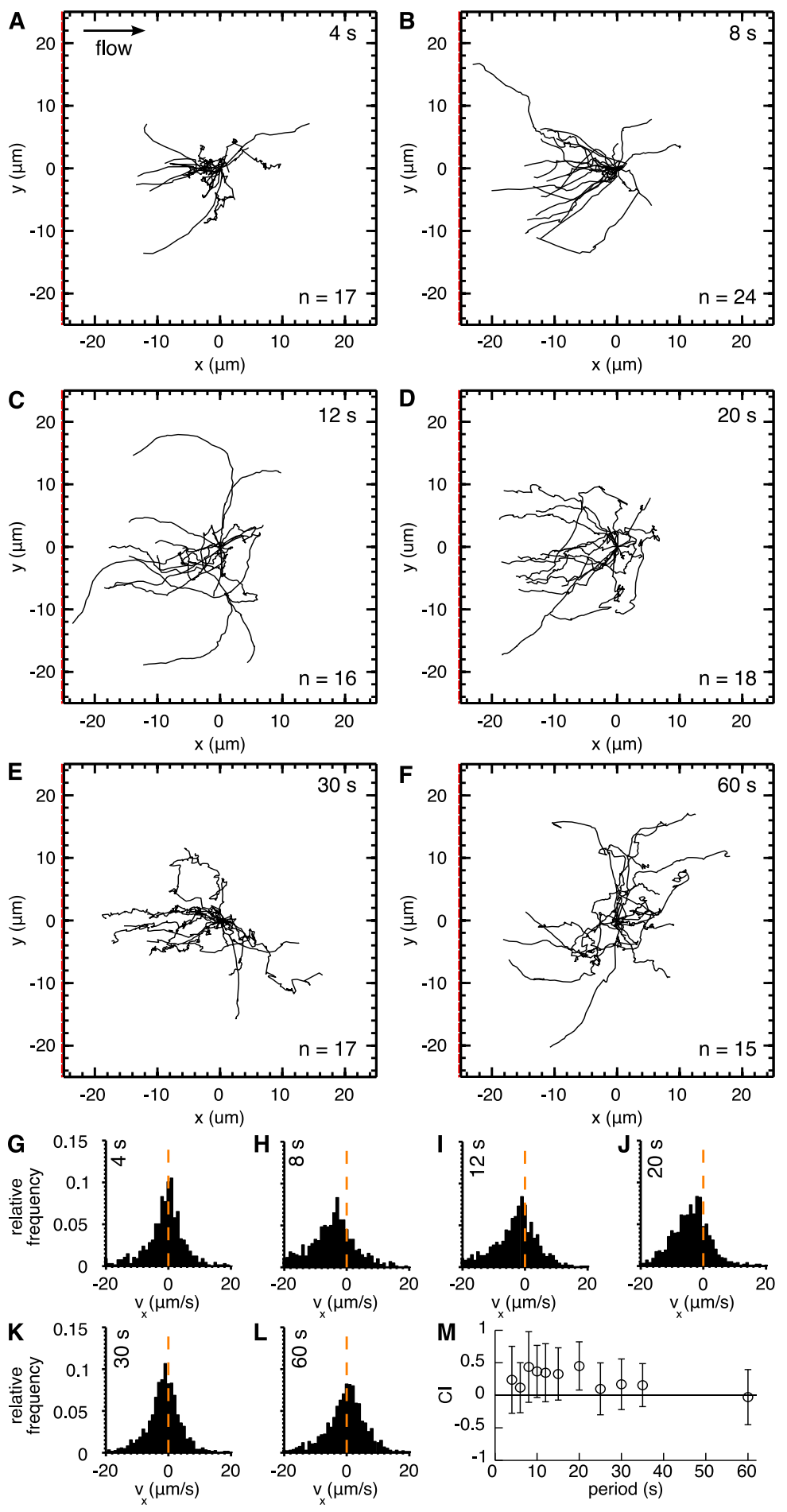

Figure 3.15.: Chemotactic response to different periodic input functions. (A-F) give the tracks of the cell centroid under stimulation, with the first position (= the time of the first stimulus application) set to $(x, y)=(0,0)$. Microfluidic flow is from left to right. The red dashed line indicates the position of the uncaging region. The input periods are given in the upper right, while the number of experiments is written in the lower right corner. (G-L) For each celltrack a distribution of the velocities in Xdirection is calculated. A bias of the histogram to negative $v_{x}$ indicates a preferenced movement towards the uncaging region. For visibility, a dashed orange line is plotted at $v_{x}=0 \mu \mathrm{m} / \mathrm{s}$. (M) The chemotactic index is plotted as a function of stimulation period). 
Note that $D$. discoideum is sensitive to near UV-light (Poff and Butler (1974), Gillies et al. (1976)) and hence it is necessary to rule out the possibility, that the cell follows the light of the uncaging pulse and not the chemotactic input. The result of the application of the $405 \mathrm{~nm}$ bleaching laser in the absence of DMNB caged cAMP is shown in 3.16 ((A) $\mathrm{T}=10 \mathrm{~s}$ and (B) $\mathrm{T}=20 \mathrm{~s}$ forcing). No bias in negative $\mathrm{x}$-direction is introduced by the bleaching laser itself to the cell movement. The chemotactic index even shows an average orientation away from the uncaging region. Finally, we can conclude that $D$. discoideum is able to orient its movement through periodic sequences of short-lived gradients.
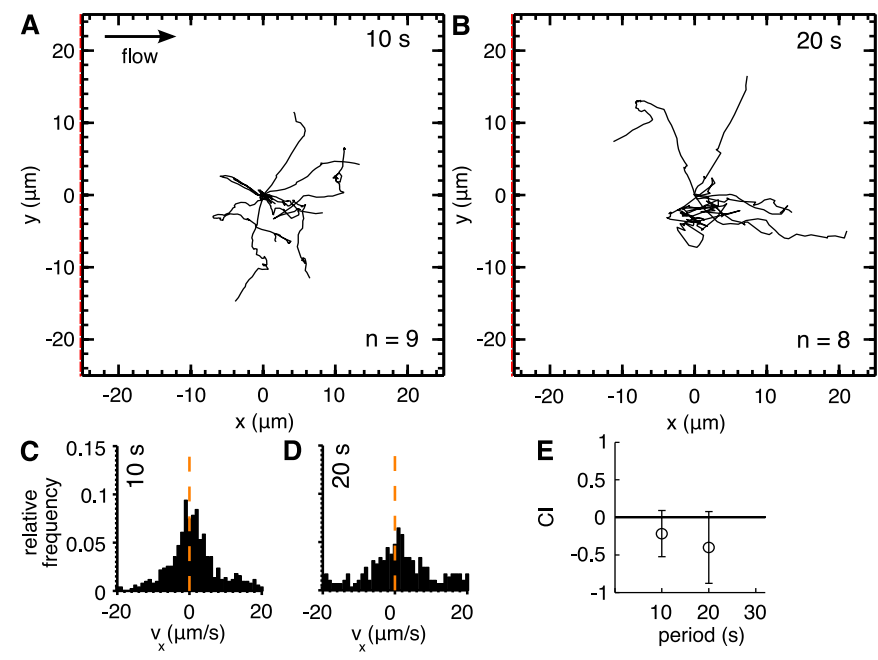

Figure 3.16.: Control of the $D$. discoideum cell movement in the absence of caged cAMP. Besides the replacement of DMNB-caged cAMP with pure PB, the setup and its parameters are the same as in the uncaging experiments. (A and B) Tracks of the centroid movement over time. Flow (black arrow) and uncaging uncaging region (red dashed line) The application of the bleaching laser itself leads to no bias of the cell movement towards the uncaging region.

\subsubsection{Discussion}

The pulse creates a gradient of $\approx 8 \%$ difference between the front and the back of the cell, which lasts for not more than $0.5 \mathrm{~s}$. A single pulse is not sufficient to impose a bias on the D. discoideum movement, while a periodic sequence of pulses fulfills this, at least in the range from $\mathrm{T}=4 \mathrm{~s}$ to $\mathrm{T}=35 \mathrm{~s}$, with varying efficiency. The orientation of the cell follows a difference in the cAMP receptor occupancies between the front and the back. Certainly, already the first pulse induces a difference of occupied receptors between front and back of the cell and engages the downstream effectors (i.e. the G-protein and Ras GTPase mediated 
signalling pathways). The binding rate of cAMP to the cAR1 receptor is about $1.1 \mathrm{~s}$ (Ueda et al., 2001), so the difference of occupied receptors vanishes practically immediately after the cAMP pulse was washed away by the flow. The activated proteins of the signal processing cascade however remain active on longer timescales (the respective intrinsic timescales of each individual effectors). Chemotactic sensing is a stochastic process and the internal gradient introduced by a single short-time pulse might just be indistinguishable from noise. Since the downstream effectors stay activated on longer timescales, subsequent pulses can fortify the internal gradient of activated proteins so at some point it becomes distinguishable from the noise.

The development of the velocity in $\mathrm{x}$-direction over time is given in figure 3.17. The more negative the $v_{x}$, the faster is the (average) migration of the cell population towards the uncaging region. Figure 3.17A shows the negative control (no external stimulation) and the positive control (continuous stimulation). The step response shows the strongest movement in negative $\mathrm{x}$-direction after $\mathrm{t}=40-80 \mathrm{~s}$. This recovers slower migration within the experimental timescale. The cells move closer to the uncaging region and, by approaching it, eventually can not distinguish the gradient anymore.

After receiving the initial stimulus the cells need $20-30$ s to orient and to start the directed migration, which is in agreement with the cringe response and the biphasic timescales (see figure 3.1C). Regarding the medium input periods ( $\mathrm{T}=10 \mathrm{~s}$ to $\mathrm{T}=20 \mathrm{~s}$ ) the cells move gradually and slower than in response to the continuous input increase their velocity in -x direction (figure 3.17B). Similar to the step response, the velocities seem to approach $0 \mu \mathrm{m} / \mathrm{min}$ the longer the experiment lasts, i.e. the closer the amoeba approaches to the cAMP source. Note, that the response to an $\mathrm{T}=8 \mathrm{~s}$ input is not plotted, because the starting value is not equal to $0 \mu \mathrm{m} / \mathrm{min}$, i.e. for some (probably statistical) reason a majority of cells already is biased towards the uncaging region and remains that feature under the periodic stimulation.

Partially, the periodicity of the external forcing appears in the total velocity as well as in the $v_{x}$ over time (for instance for $\mathrm{T}=12 \mathrm{~s}$ response, figure $3.17 \mathrm{~B}$, red). In this case, the cell slows down or even stops its movement immediately after receiving a pulse and restarts its (eventually directed) migration several seconds later. Higher periods do not change $v_{x}$ (aside from its fluctuations) or need more time to develop the biased movement. For instance for $\mathrm{T}=25 \mathrm{~s}$ the $v_{x}$ becomes slightly negative for $\mathrm{t} \geq 100 \mathrm{~s}$. This difference to the medium input periods is important. Straightforward thinking, one could assume that over the longer timescale the polarization might be lost, before it can be renewed or enhanced by the subsequent stimulus. The response to $\mathrm{T}=25 \mathrm{~s}$ (and partially to $\mathrm{T}=30 \mathrm{~s}$ ) indicate that the velocity in $\mathrm{x}$-direction might also just be function of the total number of pulses, which 
hit the cell. Within $100 \mathrm{~s}$, a cell exposed to a $\mathrm{T}=25 \mathrm{~s}$ periodic input has received 4 pulses, while shorter period input functions impose more pules on the cell. These cells therefore might much earlier beat the signal to noise ratio.

In the setup used here, the total time of the experiment is limited by the movement of the cell. In an advanced approach, one would have to use this setup mounted on a co-moving stage, which shifts the imaging region with respect to the cells centroid. Only this would ensure that the absolute number of input pulses of cAMP is the same for each input period. Aside from this experimental limitation, one can conclude that periodic pulses carrying a short lived gradient sequentially improve the signal to noise ratio of $D$. discoideum chemotactic response to a value that results in biased movement.
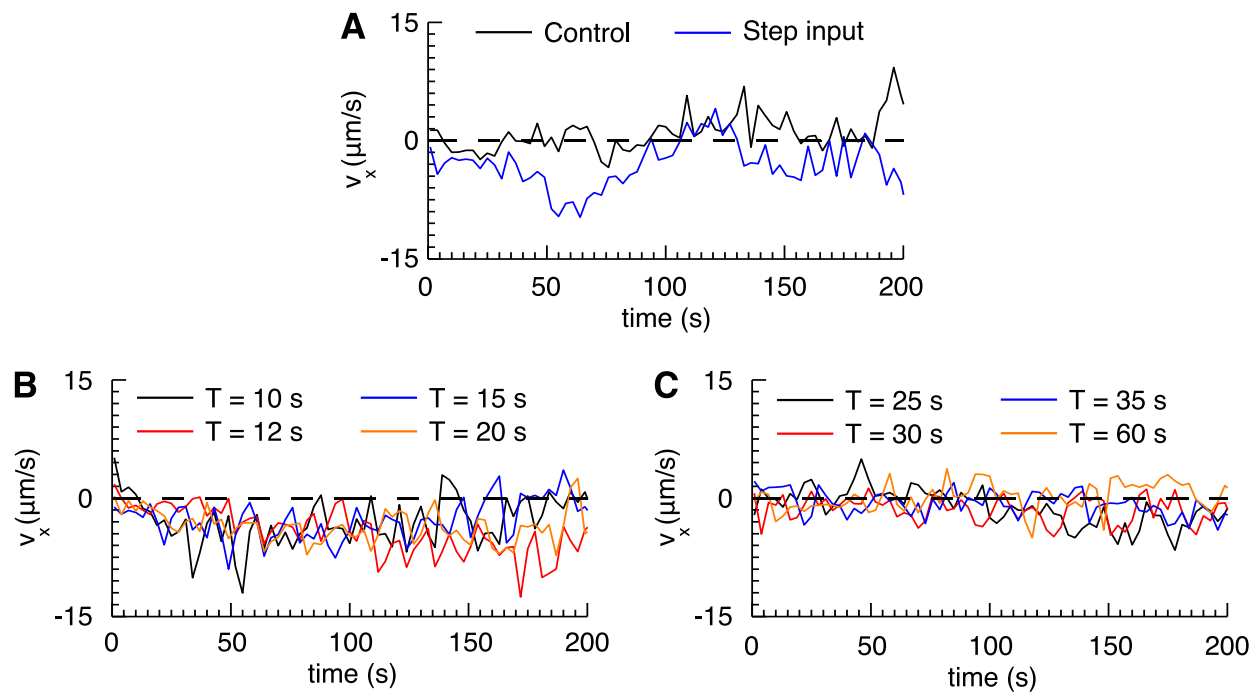

Figure 3.17.: Development of the averaged velocity over time in response to various stimulation periods. These are computed as the averages of all velocities in $\mathrm{x}$-direction at each timestep $(\Delta \mathrm{t}=3 \mathrm{~s})$. (A) compares the $v_{x}$ as a function of time of the control (black) and the step response (blue). The step response celltracks show their strongest bias to the uncaging region $40-80 \mathrm{~s}$ after the onset of the continuous stimulation. In (B) the responses to medium periodic input are given. Those develop a stronger bias towards the uncaging region over a time of 2 minutes under forcing and eventually recover a non biased movement towards the end of an experiment. In contrast, the velocities in $\mathrm{x}$ direction of cells forced with long input periods show no or a slowly developing bias towards the uncaging region. As the chemotactic index indicates, the response to a $T=60 \mathrm{~s}$ response signal shows a time dependent velocity in $\mathrm{x}$ direction which fluctuates around $0 \mu \mathrm{m} / \mathrm{min}$

So far only the $\mathrm{x}$-coordinate carried information, but is $D$. discoideum able to read out a distortion of the isotropy along the y-direction? This question was investigated together with Rabea Sandmann in the framework of her Bachelor thesis (Sandmann, 2009). The 
adapted uncaging setup draws the uncaging region tilted with respect to the flow direction. This changes the isotropy in y-direction of the concentration time profile. The changed setup is sketched in figure 3.18A. The concentration profiles were evaluated using finite element simulations (figure 3.18B) at positions marked by the respectively colored squares in figure 3.18A. The experiments were initially carried out in approximatly $50 * 50 \mu \mathrm{m}$ sized imaging region with the uncaging tilted by $25^{\circ}$ and $41^{\circ}$. The responses to a $41^{\circ}$ tilted line showed a clear bias towards the upper left corner of the imaging region. This could not be obtained under $25^{\circ}$ tilted periodic stimulation. However it appeared that the cells turn their movement towards the tilted line on a longer timescale. The size of the imaging region was changed to approx. $100 * 50 \mu \mathrm{m}$, which was a direct consequence of the previously obtained results. The distance to the uncaging region was set to $40 \mu \mathrm{m}$. On this length and time scale, one can clearly see that, on average, the cells orient with respect to the source orientation (figure 3.18C). The majority of the celltrack end positions (blue triangles) are found in the upper left quadrant of the imaging region. Therefore one can state, that the periodic sequence of tilted pulses and the carried information about the source orientation in x and y can be read out by the $D$. discoideum chemotactic system.

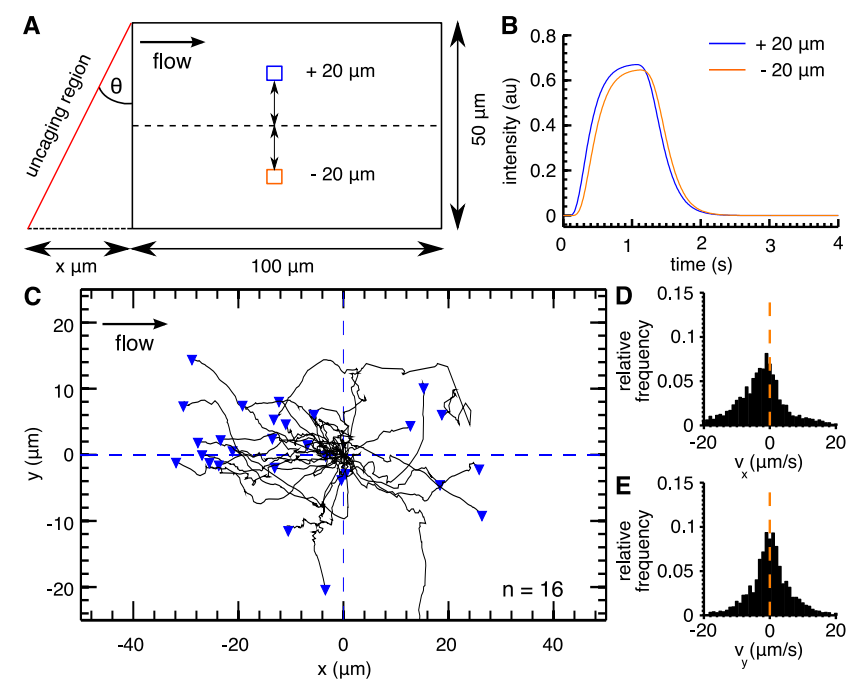

Figure 3.18.: Chemotactic response to an additionally introduced gradient. (A) shows the variation of the uncaging setup. The gradient is introduced in y direction by tilting the uncaging line (red) with respect to the flow direction. The angle $\theta$ was fixed to $25^{\circ}$. Note that also the size of the imaging region was changed to approximatly $100 * 50 \mu \mathrm{m}$ (B) FEM simulation evaluated the difference of cAMP concentration, between the position marked by the black squares in (A). The celltracks of 30 independent experiments are overlayed in $(\mathrm{C})$. The starting position is set to $(\mathrm{x}, \mathrm{y})=(0,0)$, while the endposition of each individual track is highlighted by the blue triangles. The velocity distributions in $\mathrm{x}$ and $\mathrm{y}$ are plotted as histograms in (D) and (E). The orange dashed line marks $\mathrm{v}=0 \mu \mathrm{m} / \mathrm{min}$. 
CHAPTER 4

\section{On the existence of self-sustained cytoskeletal oscillations}

In the previous chapter we learned about the oscillatory properties of the actin cytoskeleton under external driving. We will now draw our attention towards the SCAR-complex, a regulator of the actin cytoskeleton, which couples the action of the Arp2/3-complex to the chemotactic sensory system. Disrupting the different members of the SCAR-complex has lead to insight into the contribution and role of the complex in the regulation and signal transduction pathway of the Dictyostelium chemotaxis system as well as other chemotaxis systems (Ibarra et al. (2005), Insall and Machesky (2009)). We focused our attention on the double knockout of the proteins SCAR and PIR121, first described by Blagg et al. (2003). Confocal laser scanning microscopy recordings of the LimE-GFP tagged knockout revealed a presumably self-sustained oscillation of the cortical and cytosolic fluorescence intensity over time. This indicates repetitive cycles of actin polymerization and depolymerization in the presence of an impaired SCAR-complex. The occurrence of these oscillations is found for $60 \%-70 \%$ of all cells and is therefore considerably higher than in the LimE-GFP labeled AX2 strain discussed in the previous chapter. However, these oscillations could only be obtained using confocal laser scanning microscopy and lacked verification by other microscopy techniques such as spinning disk microscopy (Ishikawa-Ankerhold, 2008). As a consequence a light dependence of the autonomous oscillation has been proposed (Gerisch, 2008). Within this work I have investigated possible confocal and optical effects on the oscillation. Furthermore, non-optical approaches and techniques were applied to exclude any artificial effects of light. Finally we will focus on the question of whether the fluorescence intensity oscillations are self-sustained or a transient type of behavior. 


\subsection{Self-sustained actin oscillations in SCAR(-)/PIR121(-) LimE-GFP}

\subsubsection{The SCAR complex and knockouts of its members}

Within the signal transduction pathway of many eukaryotic organisms the WASp proteins regulate the function of Arp2/3-complex (see Veltman and Insall (2010) for a recent overview). The Dictyostelium SCAR protein is genetically a member of the WASP protein family. The knockout mutant restores the phenotype of a cAR2 receptor mutant ${ }^{1}$. This earned the protein the name: Suppressor of cAMP receptor 2 (Bear et al., 1998). The SCAR protein itself is capable of activating the Arp2/3 complex in a WASP related manner (Machesky and Insall (1998), Machesky et al. (1999)). However, it is only one out of five proteins forming the SCAR-complex. The other proteins are PIR121 (p53 inducable RNA), NAP1 (Non-catalytic region of tyrosine kinase adaptor protein (Nck)-associated protein), HSPC300 (hematopoietic stem-cell progenitor) and Abi (Abelson murine leukemia oncogene 1 (Abl)-interactor) (Insall and Machesky, 2009). Consequently, knockout mutants of all members have been produced in order to investigate each proteins specific role in the complex. Besides the recovery of tip formation in a cAR2(-) background, the disruption of the SCAR gene results in a mutant with decreased levels of filamentous actin and impairments in movement. The PIR121(-) phenotype consists of artificially large cells with a strongly decreased SCAR protein content and an increased concentration of filamentous actin (Blagg et al., 2003). The double knockout of the SCAR and the PIR121 protein, investigated here in more detail, shows a phenotype with high similarities to the SCAR(-) phenotype. This indicates that the action of PIR121 is mediated via the SCAR protein (Blagg et al., 2003). PIR121 is therefore located upstream in the signalling cascade and furthermore it prevents the SCAR protein from being degraded (Blagg et al. (2003), Kunda et al. (2003), Steffen et al. (2004)). The actual regulation of the SCAR-complex is still not fully resolved, but recently the structure of the wave regulatory complex (the mammalian analog to the SCAR-complex) was revealed (Chen et al., 2010) and the following model of activation has been proposed. The SCAR-complex remains in an auto-inhibited state upon activation. The acidic domain of the SCAR-complex, which interacts with the Arp2/3 complex binds to the PIR121/NAP1/Abi complex (Chen et al. (2010), Davidson and Insall (2011)). The Rho-GTPase Rac1 binds to the PIR121 protein and activates the complex (Eden et al. (2002), Innocenti et al. (2004)). Upon activation the acidic domain is free to interact with the Arp2/3-complex. This transition is sketched in figure 4.1.

\footnotetext{
${ }^{1}$ In the cAR2(-) knockout the development arrests before the tip formation starts (Bear et al., 1998).
} 


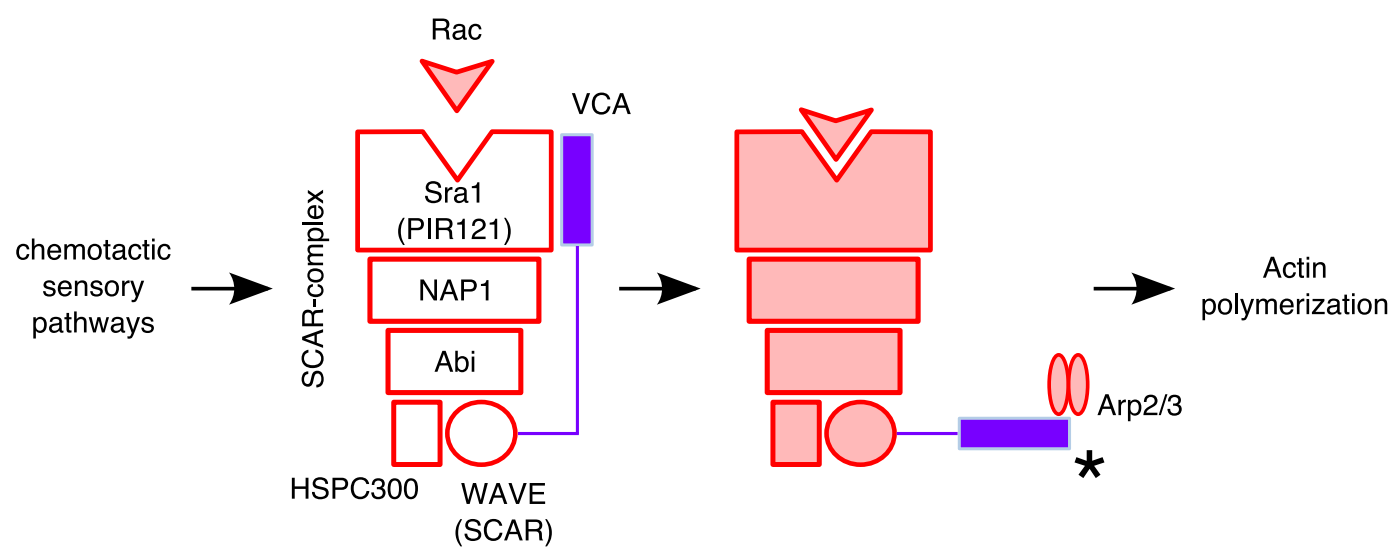

Figure 4.1.: Sketch of the model proposed for the activation of the SCAR-complex. The RhoGTPase Rac couples the SCAR complex to the chemotactic sensory pathways. The active Rac binds to the PIR121 protein and activates the, so far, inactive complex. The complex is inactive since the acidic domain of the SCAR protein (drawn in purple) is not free to interact with Arp2/3. The binding of the Rho-GTPase to the PIR121 protein frees this domain and consequently actin polymerization and branching are engaged via the active Arp2/3. Please note that this model was proposed for the mammalian analog of the SCAR-complex. If the names of the member proteins differ, then in parenthesis the nomenclature in Dictyostelium discoideum is given. The figure has been adapted from (Blagg and Insall (2004), Innocenti et al. (2004), Ismail et al. (2009)).

\subsubsection{Detected oscillations in the cortical actin network}

Self-sustained oscillations of the filamentous actin network have been observed in a SCAR(-) /PIR121(-) strain, which additionally carries the LimE-GFP label. The genetic labeling and the characterization of the mutant were carried out by Hellen C. Ishikawa-Ankerhold as part of her $\mathrm{PhD}$ thesis within the lab of Günther Gerisch. The free running oscillations showed a period distribution with a peak at $20 \mathrm{~s}$. This accounts for all three mutants, i.e. the two single gene knockouts as well as the SCAR(-)/PIR121(-) double knockout. Although the frequency distribution was similar, the amplitude of the oscillation was considerably higher in the double knockout. From these observations it was concluded that the SCAR protein prevents the transition from steady state polymerization to alternating phases of polymerization and depolymerization (Ishikawa-Ankerhold, 2008). However, an attempt to characterize the oscillations in 3 dimensions using the spinning disk microscopy failed to visualize the oscillations. The dependence on the used microscopy technique raised the question of whether the magnitude of the light intensity changes the properties of the oscillation or even induces it; D. discoideum expresses blue light photoreceptors (Poff et al., 1974), which might respond to the scanning laser. We will discuss this question in the subsequent section and then characterize the oscillation in more detail. 


\subsection{Dependence of the cortical actin oscillations on different conditions}

\subsubsection{Dependence on light intensity}

To investigate possible influences of the intensity of the imaging laser on the oscillations, the experiments of Hellen C. Ishikawa-Ankerhold (2008) were expanded as follows: Starved AX3 SCAR(-)/PIR(-) LimE-GFP cells were placed on the glass bottom of a Matek Petri dish and imaged with confocal laser scanning microscopy. The intensity of the $488 \mathrm{~nm}$ wavelength Ar-laser was varied between 10 and $40 \%$. The lower bound was set to $10 \%$ to minimize possible light induced artifacts, while maintaining a reasonably good signal to noise ratio. The upper intensity level was set to $40 \%$. Above $40 \%$ photobleaching and phototoxicity could potentially influence the cell over the duration of an experiment (see the materials and methods section 2.3 for the characterization of the microscope).

Two representative recordings with different laser intensities are shown in figure 4.2A and B. It is apparent, that the oscillation occurs in both cases, but is not necessarily stable over time; the oscillation is not always distinguishable from the background. Therefore each signal recorded with confocal microscopy is subject to windowing. For each window the autocovarriance and spectral analysis are performed as described in the materials and methods section 2.5. An example window is indicated in the timedomain plots of figure 4.2. The respective calculated spectra are given in the following plots. A signal was counted as oscillating, if at least in one window the oscillation criteria were fulfilled. The autocovariances of each window results (if it passes the criteria) in one single period. For each entire signal an average is computed and the distribution of these averages in relation to the imaging laser intensity is given in figure 4.2E. The FFT based detection method allows several detections per signal (i.e. several modes with a normalized spectral power $>0.1$ ). Therefore no average per signal is possible and each oscillatory window and its dominant frequencies are counted. The histogram (figure 4.2F) consequently needs to be normalized by the number of counts. As one can clearly see, the histograms almost equal each other. We therefore conclude that the oscillation is not dependent on the laser intensity, at least within the applied range. Both detection and quantification methods show a peak of the period distribution at approximately $\mathrm{T}=12 \mathrm{~s}(\mathrm{f}=0.083 \mathrm{~Hz})$. This is considerably different to the previously obtained results, which determined the peak at $\mathrm{T}=20 \mathrm{~s}$ (Ishikawa-Ankerhold, 2008). However, here the range of detected periods includes periods as small as $8 \mathrm{~s}$ and reaches up to $20 \mathrm{~s}$. Within the spectrum based detection, low frequencies dominate the histogram. 

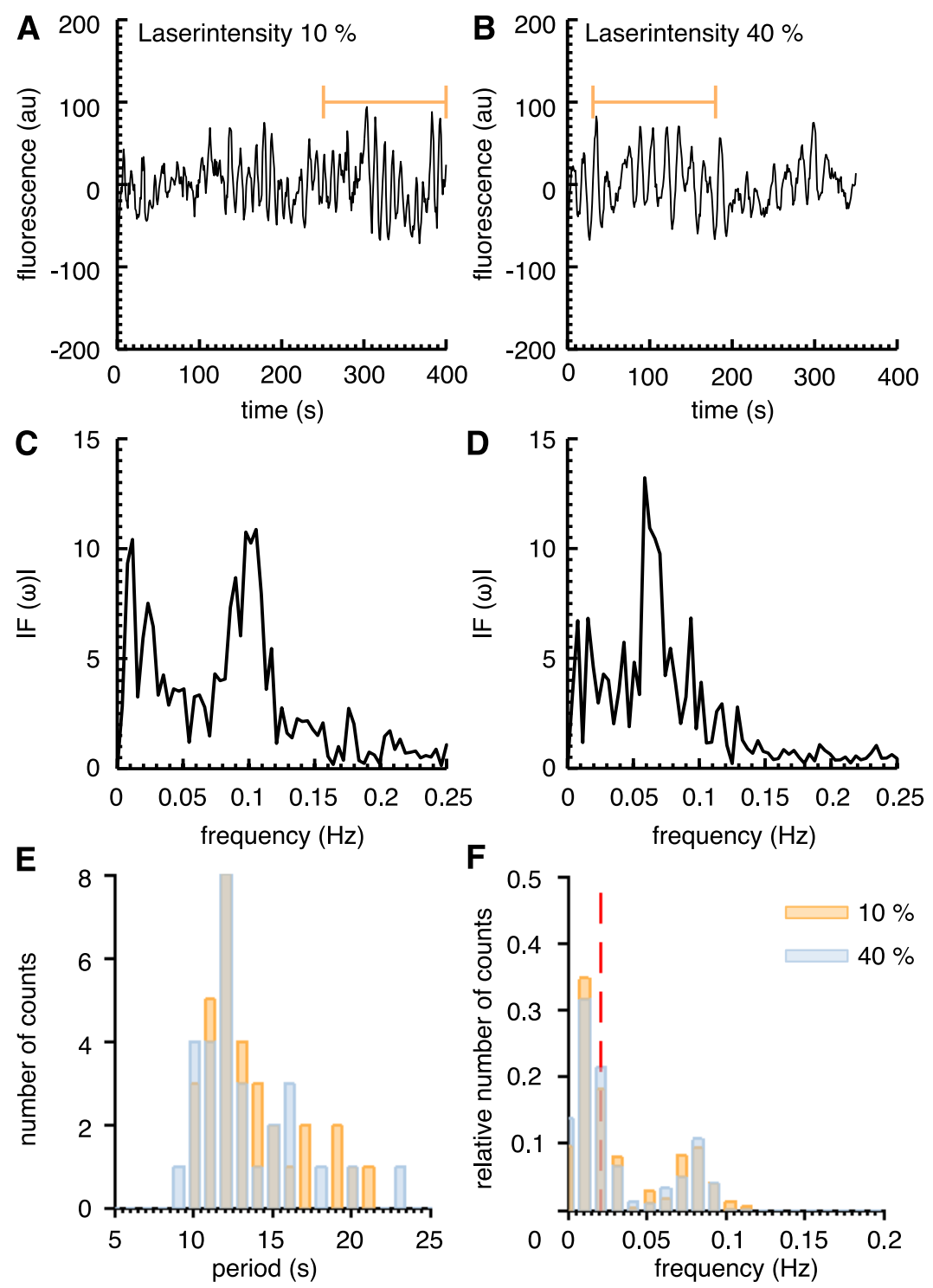

Figure 4.2.: Examples of autonomous oscillations observed with different laser intensities of the confocal scanning laser. Top plots show the oscillations in the normalized cytosolic fluorescence signals recorded at $10 \%$ (A) and $40 \%$ (B) of the available laser intensity. (C) and (D) give the frequency spectrum of a windowed part of the signals in (A) and (B) respectively. The window position is indicated by the orange horizontal line in the top plots. The comparison of all detected periods in cells irradiated with $10 \%$ and $40 \%$ laser intensity is given in (E) and (F). (E) summarizes the detected periods by the regularity and distance of the autocovarriance zerocrossings. Each has been windowed and out of each window with a positive detection an averaged period is calculated. Orange bars represent the detected periods for $10 \%$ laser intensity and blue bars the detected periods under $40 \%$ intensity, respectively. The windowed cytosolic signals were subject to a FFT and each peak larger than 0.1 normalized power is counted as an oscillation (F). Every windowed result is included in the histogram, which is normalized by the number of its elements. Colors represent the different laser intensities as in (E). The horizontal dashed red line indicates the frequency, which allows 4 full cycles of the oscillation within the window extension. Positive detections smaller than this frequency belong to long term trends in the signal. In (E) and (F) the two applied laser intensities are not distinguishable. A dependence on laser intensities in this range can be excluded. 
These low frequency detections can not be considered to be oscillatory, since less than 4 full periods are observed within the sampled window. They are more likely to belong to long range trends. The possibility that the light of the laser beam induces the oscillations was ruled out by Hellen Ishikawa-Ankerhold already ${ }^{2}$ (Ishikawa-Ankerhold, 2008). Now we can furthermore conclude that the autonomous oscillations of the LimE-GFP in the SCAR(-)/PIR121(-) knockout are not influenced by the light intensity.

\subsubsection{Persistence of the oscillations over time}

Temporarily interrupted oscillations make it necessary to use windowing and short-time Fourier transforms. We could asked ourselves: Are these interruptions intrinsic or artificial? If transient oscillation had a finite time extension, they may be an intrinsic property, however, 3D movement of the cell could produce artificial oscillations. Due to confocal imaging a drastic shape change could lead to strong changes in the fluorescence time profile, which might cover an instantaneous fluorescence intensity oscillation. I approached this question by fitting a single frequency sinusoid to one part of the interrupted oscillation. Extending the fitted sine to the artificially chosen second part allows comparison of the phases in both. Figure 4.3A gives the control: a non-interrupted oscillation. The orange vertical bars give the interval of the oscillation to which the sinusoid (bottom plot, red) is fitted. The second part of the oscillation (interval indicated by blue vertical bars) is compared to this sinusoid. Both cropped signals were detrended prior to comparison. In the case of this ongoing and non-interrupted oscillation, the two compared parts oscillate in phase. Therefore we can assume that the oscillation, if stable, does not change its frequency over the course of an experiment. This is further confirmed by the frequency spectra, which indicate a stable frequency over time for the oscillations (data not shown). One has to conclude that an intrinsically ongoing, but temporarily non visible oscillation also has the same phase at each time position. In figure 4.3B - C we see two different examples of temporarily interrupted oscillations of the cytosolic LimE-GFP fluorescence. All cases of phase relationship, i.e. in phase, phase shifted and out of phase by 90 degrees are observed. These two figures show exemplary cases. The fact that all cases are present can therefore not exclude the possibility of starting and stopping (i.e. transient) oscillations.

\footnotetext{
${ }^{2}$ The phase of the oscillation is different from cell to cell and not the same as one would expect, if the oscillation is induced by the light of the microscope.
} 


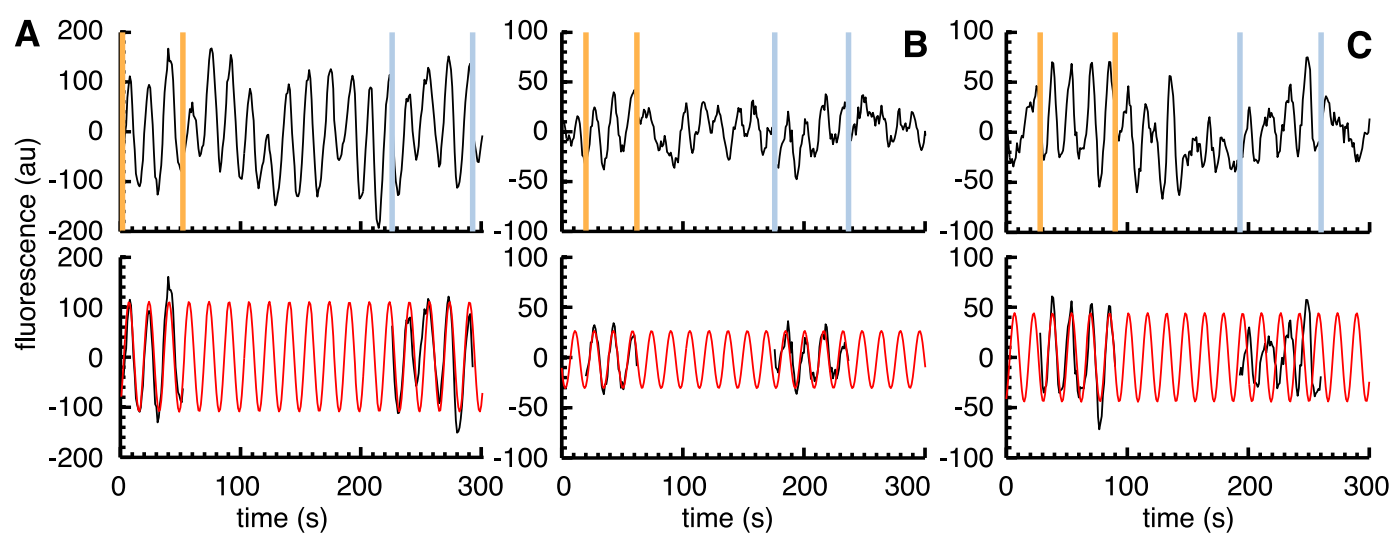

Figure 4.3.: Analysis of temporarily interrupted oscillations. (A) to (C) top plots show the recorded oscillation examples of the cytosolic fluorescence intensity. The orange vertical lines indicate the time region to which a single frequency sinusoid is fitted. The sinusoid is extended to the full range of the signal and therefore also to the second oscillatory region (indicated by blue vertical lines). Comparisons of the sinusoid and the detrended oscillatory regions are given in the respective bottom plots. (A) shows a control of a not interupted oscillation. The signal remains in the same phase over the recorded experiment. In (B) and (C) each example shows an oscillation, which becomes temporarily indistinguishable from noise and reappears afterwards. Both cases: in phase (example B) and phase shifted (example C) occurred among the total set of experiments.

\subsubsection{Dependence on the confocal plane position}

$60-70 \%$ of all SCAR(-)/PIR121(-) show temporary or sustained oscillations in the fluorescence intensity. In this subsection we will address the question as to whether the cortical actin oscillation is to some extent spatially restricted. Confocal microscopy images sections over a limited small thickness. This may be why $30-40 \%$ of all observed cells are not observed to oscillate autonomously and why sudden disruptions in the oscillations are observed. In figure 4.4 three examples of cells recorded in 2 different focal planes are shown. The planes are separated by $2 \mu \mathrm{m}$. The top plots show the temporal evolution of the cytosolic fluorescence signals. As discussed in the previous section, windowing is applied to obtain the short-time Fourier transform. The phase of the dominant frequency peak within each window is displayed in the bottom plots. In more than $90 \%$ of all cases the oscillation was observed in both focal planes. Figures 4.4A and B show two representative time series where the oscillation is present in both recorded focal planes. In most cases the oscillation was in phase among both planes. Figure $4.4 \mathrm{C}$ shows an example oscillation where the oscillations in the two focal planes are out of phase with respect to each other. In rare cases the oscillation is observed in one plane and not detected in the other. 

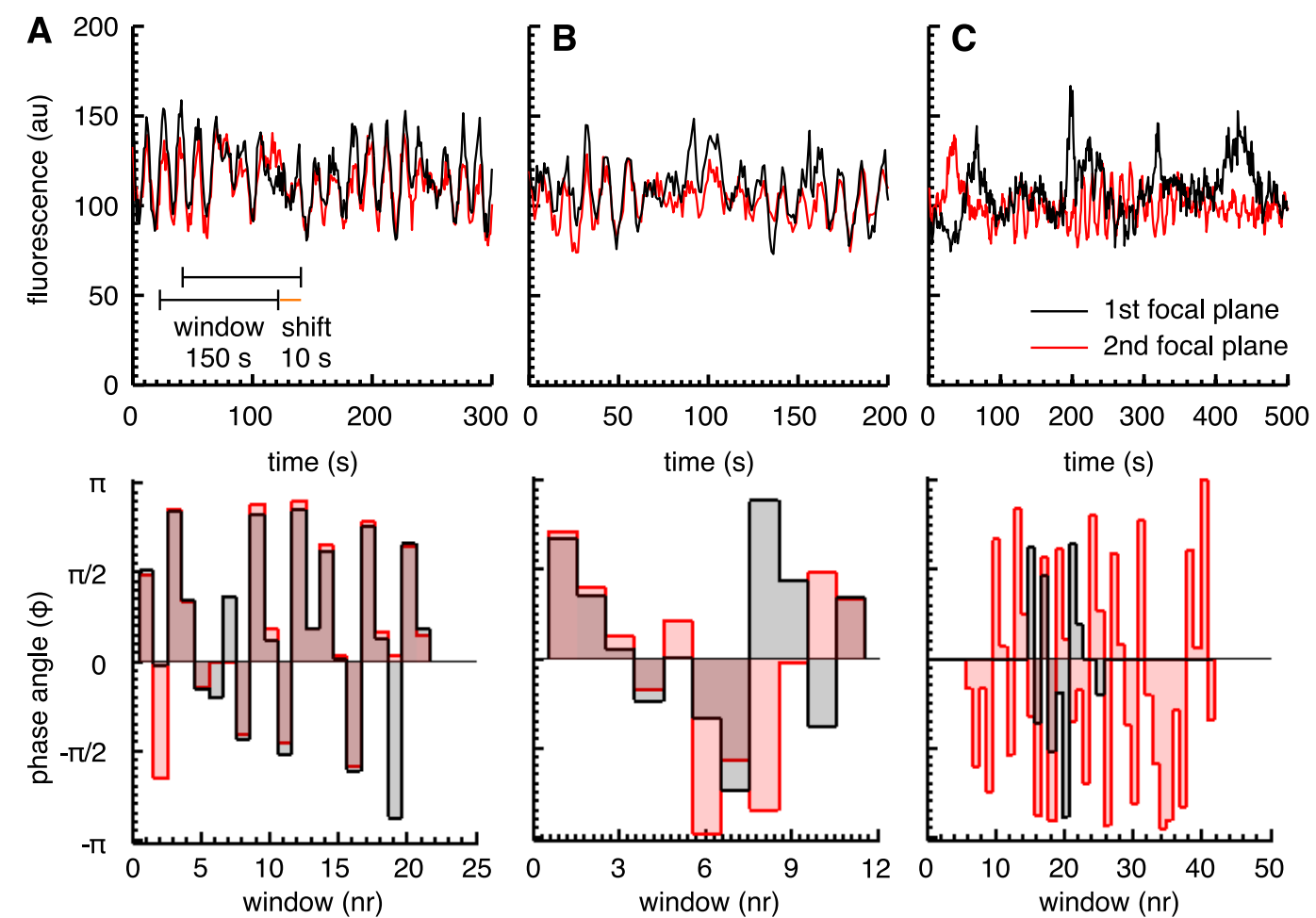

Figure 4.4.: Two confocal crossections recorded for one cell. The top plots show three examples of observed oscillatory patterns. In more than $90 \%$ of all experiments the oscillation was present in both focal planes (A and B), while in rare cases it is only detected in one plane (C, less than $10 \%$ of all cells). In the corresponding bottom plots the phase of the dominant frequency peak is displayed as a function of the window number. Windowing is applied to compute the short time fourier transform. Each window is $150 \mathrm{~s}$ long and differs by $10 \mathrm{~s}$ from the subsequent window (as sketched in (A)).

The obtained results lead to the conclusion, that the observed fluorescence intensity oscillation occurs globally throughout the cell body. This gives the possibility for non-optical AFM based whole cell measurements discussed in the following section.

\subsection{Non-optical detection of self-sustained oscillations}

The intensity oscillations can be thought of as periodic shape changes induced by actin polymerization. These shape changes are non-optically measurable parameters. In collaboration with Marco Tarantola (MPI DS) and Edith Schäfer (Faculty of Chemistry, University of Göttingen), the shape changes were investigated using non-optical methods: electrical 
cell-substrate impedance sensing (ECIS) and atomic force microscopy (AFM). The methods are shortly summarized and the results discussed.

The ECIS method is based on a gold electrode on which the cells are located (immersed in the respective culture medium). The observable parameter is the electrical impedance, as it changes with the current flow through the medium and the insulating properties of the cells cultured on top of the electrode. Therefore these measurements give hints about the coverage of the gold electrode and therefore about the cell shape (Giaever and Keese, 1993). In general, this method is applied to confluent layers of cells (Lovelady et al., 2007). For our application only single cell measurements can be used to ensure reliable results. Since the oscillations that occur are intrinsic to each cell and do not synchronize when multiple cells are present, additional cells will not increase the signal strength but only make the signal more noisy. A single cell gives a clear shift in the impedance while entering the surface of the electrode and the impedance signal can be compared to the cells actual shape (Schaefer et al., 2011). Shape changes due to repetitive polymerization cycles are too small to be distinguishable from movement of the cell. ECIS has been used however to successfully detect the cAMP induced oscillatory shape changes due to the aggregation of Dictyostelium discoideum (Schaefer et al., 2012).

For single cell measurements it is perhaps more appropriate to focus on atomic force microscopy as a method to monitor shape changes. AFM is a high-resolution technique which allows the topography and composition of a surface to be images. It works by measuring the interactions between the sample and a tip mounted on the end of a cantilever. Images are derived from measurements of the attractive force between the tip and the sample due to deflections of the tip as it is scanned across the surface. Here however a tipless cantilever was used to monitor fluctuations in the cell. In this case, two modes of AFM operation are possible, where either the applied force or the height of the cantilever was kept constant. Within the constant height mode, strong force fluctuation may loosen the contact of the cell membrane to the cantilever in irregular intervals. These rupturing events are unavoidable and make it impossible to record time series of an appropriate length. Therefore, only constant force mode was used here. The force between the cell and cantilever was kept constant at $100 \mathrm{pN}^{3}$ and the resulting height fluctuations recorded over time. The results of these experiments (carried out by Marco Tarantola) are displayed in figure 4.5A. The SCAR(-)/PIR121(-) LimE-GFP (AX3 background) cells appear to show larger fluctuations (top plot) compared to the LimE-GFP strain (AX2 background). Nonlinear detrending 4 was applied to subtract long scale trends. The detrended signals are given in figure $4.5 \mathrm{~B}$

\footnotetext{
${ }^{3} 100 \mathrm{pN}$ was the lowest force between the cell and cantilever.

${ }^{4}$ A third order polynomial Savitzky-Golay smoothed signal was created and subtracted from the original signal.
} 
and the variance of each of these signals is calculated. The variances are compared in figure 4.5. The SCAR(-)/PIR121(-) LimE-GFP cells show a larger variance compared to the LimE-GFP, whose variances were in 4 out of 5 cases close to 0 . Oscillatory behavior is not apparent in the SCAR(-)/PIR121(-) knockout using this technique. However, the difference between the two strains can be quantified by looking at the difference in the strength of the cell height fluctuations. The difference between the AX3 wildtype and the SCAR(-)/PIR121(-) knockout needs to be studied in addition to the experiments above to rule out any strain influences.
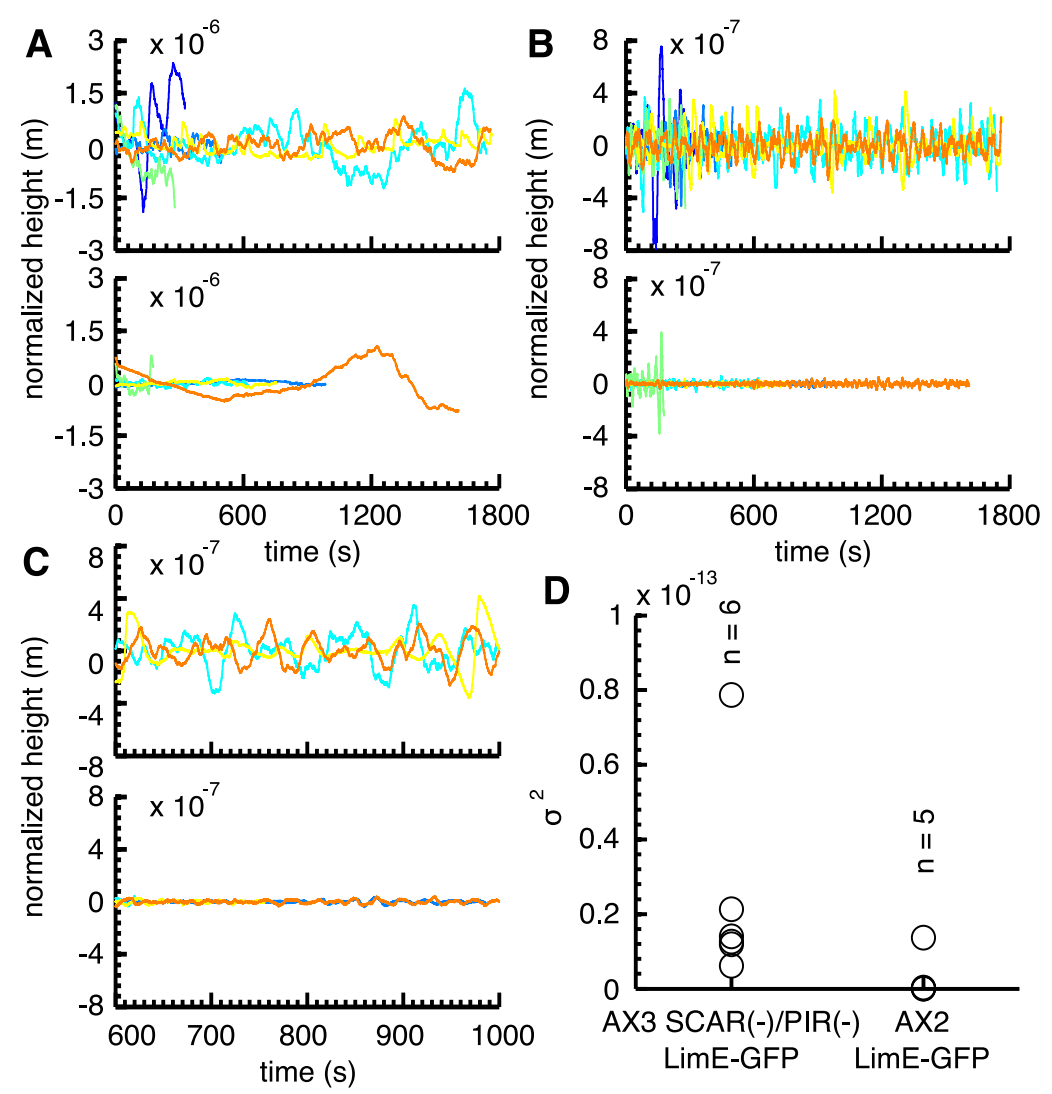

Figure 4.5.: Non-optical phenotype of the AX3 SCAR(-)/PIR121(-) double knockout recorded with atomic force microscopy. Constant force AFM was the most reliable method to monitor the differences between the two strains. The top plot of (A) shows the height changes over time for 6 different SCAR(-)/PIR121(-) cells under the tipless cantilever. The bottom plot contains the results acquired from 5 AX2 LimE-GFP cells. A local 3rd degree polynomial is fitted to the time series and is subtracted from the signal in order to remove long range trends (B). A zoom into the time traces is given in (C) for better visualization. Different colors mark individual single cell experiments in (A-C). The variances of the entire signals are then compared in (D). The AX3 SCAR(-)/PIR121(-) LimE-GFP knockout shows higher fluctuations than the AX2 LimE-GFP strain at comparable contact forces of $100 \mathrm{pN}$ (Experiments performed by Marco Tarantola). 


\subsection{Periodic stimulation of the SCAR(-)/PIR121(-) knockout}

The experimental method for periodic stimulation used here to observe the autonomous oscillatory patterns in AX3 SCAR(-)/PIR121(-) LimE-GFP was the same as that described in chapter 3. However, in this case the first pulse was applied after $60 \mathrm{~s}$ instead of $30 \mathrm{~s}$. Figure 4.6 shows the LimE-GFP response in the mutant cell to a $\mathrm{T}=20 \mathrm{~s}$ and $30 \mathrm{~s}$ input. Note that the autonomous oscillation occurs within a single cell and if present is not necessarily in phase between the different cells. Therefore the averaged signal before stimulation appears to be quiescent ${ }^{5}$. The patterns look very similar to the temporal pattern obtained by LimE-GFP wildtype forcing. This indicates that the LimE-GFP label and its associated proteins can still respond with repeated cycles of translocation and diffusion although the SCAR-complex is impaired. This is in agreement with Blagg et al. (2003), who observed that the biphasic response profile of the filamentous actin content in response to a cAMP stimulus is still present in a SCAR(-)/PIR121(-) mutant.
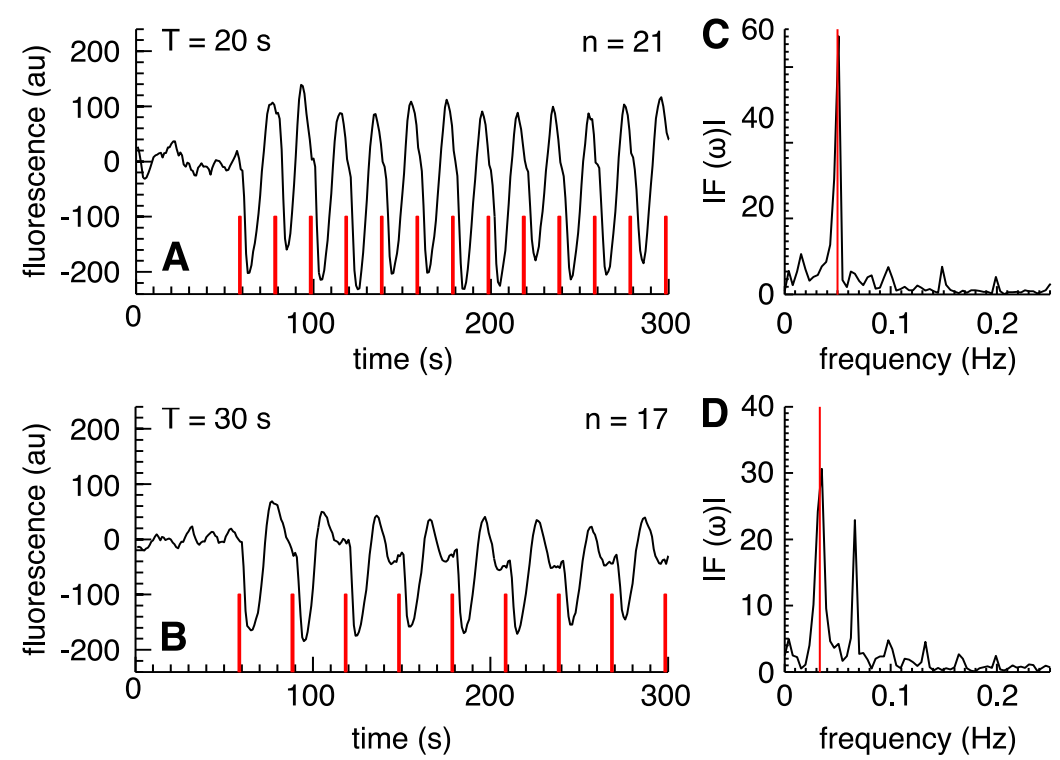

Figure 4.6.: Response of LimE-GFP in AX3 SCAR(-)/PIR121(-) to periodic stimulation. Averaged cytosolic LimE-GFP fluorescence response to $20 \mathrm{~s} \mathrm{(A)} \mathrm{and} 30 \mathrm{~s}$ (B) periodic stimulation. The red lines indicate the time of the stimulus application. (C) and (D) show the corresponding frequency spectra of the steady state responses with the forcing frequency marked in red.

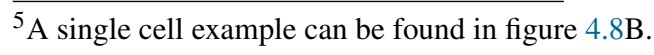


The 30 s periodic response clearly shows a peak at the second harmonic frequency. Contrary to the forcing of the LimE-GFP labeled wildtype the dominant mode is still the first harmonic frequency (figure 4.6D and compare to figure 3.5G).

Therefore in figure 4.7 the two temporal patterns are investigated in more detail. (A) compares the cytosolic LimE-GFP fluorescence averaged responses to a 30 s input (black - AX2 LimE-GFP, blue - AX3 SCAR(-)/PIR121(-)). In the knockout, the polymerization of new filamentous actin appears at exactly the same time as in the wildtype but the depolymerization peak is prolonged. The overshoot of LimE-GFP is longer than in the wildtype. On application of the subsequent pulse, the two signals come into phase with each other. This feature is more prominent in the autocovariance plot (figure 4.7B). The second smaller gain of correlation is missing in the mutant, indicating that the second translocation of LimEGFP to the cell cortex either decreases or vanishes.
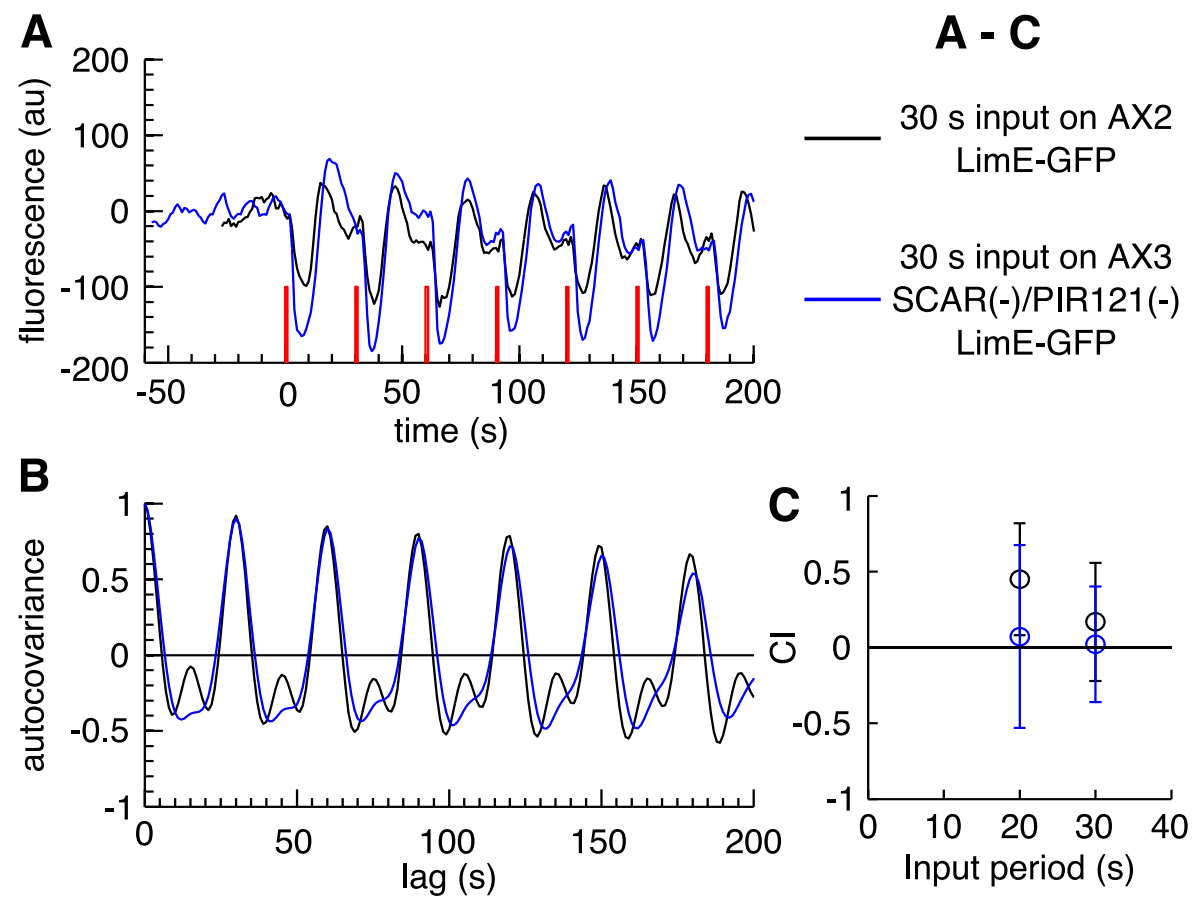

Figure 4.7.: Comparison of the wildtype and SCAR(-)/PIR121(-) mutant responses to periodic forcing of $\mathrm{T}=30 \mathrm{~s}$ input. (A) Overlay of the cytosolic AX2 LimE-GFP and the cytosolic AX3 SCAR(-)/PIR121(-) LimE-GFP fluorescence time profile. $\mathrm{t}=0 \mathrm{~s}$ corresponds to the time at which the first stimulus was applied. The respective autocovariances of both timeseries are shown in (B). (C) compares the chemotactic indices of the two strains in response to $20 \mathrm{~s}$ and $30 \mathrm{~s}$ forcing. 
The LimE-GFP label is known as a filamentous actin marker (Schneider et al., 2003), but essentially associates with the GTP bound form of Rac1A (the activator of the SCAR complex (see figure 4.1)). The Rac1A and the LimE protein show comparable translocation profiles to the cortex (Filic et al., 2012). Hence our conclusions might therefore be restricted to the Rac1A-GTPase. It could be that Rac1A's retranslocation from the cortex is actually more prolonged. The SCAR(-)/PIR121(-) cells are also impaired in their chemotactic efficiency towards the periodically occurring source of cAMP, as can be seen from the chemotactic index ${ }^{6}$ in figure 4.7C (see also Blagg et al. (2003)).

\subsection{Discussion}

Spontaneous oscillations are wide spread in biology and have gained attention, as they are a simple representation of complex dynamics and suited for experimental and theoretical study (Kruse and Juelicher, 2005). Here, I have repeated and refined the characterization of the LimE-GFP label in a SCAR(-)/PIR121(-) background. The spontaneous oscillation was observed using confocal laser scanning microscopy. Results indicate that no dependence on the intensity of imaging laser nor the position of the confocal plane exist. AFM height measurements indicate stronger fluctuations of the cell height in the double knockout mutants. One explanation for this is an impaired actin cortex with a higher rate of rebuilding and depolymerizing of the actin filaments, leading to higher fluctuations of the cell height. It should be mentioned that the readout of the tipless cantilever does not include any spatial information about possible fluctuations, neither does ECIS.

The data for $D$. discoideum indicate the oscillation is not unique to the double knock-out mutant (see section 3.1). Up to $8 \%$ of all wildtype LimE-GFP cells showed periodic oscillations without external stimuli, as well ${ }^{7}$. However, autonomous LimE-GFP oscillations had been observed with a much higher frequency in the SCAR(-)/PIR121(-) double knockout background. In the previous chapter we raised the question, as to whether the actin regulatory system is close to an oscillatory instability. Taking this into consideration, it could be argued that the absence of the SCAR and PIR121 proteins in the actin regulatory system shifts it closer to the instability. In $60-70 \%$ even passes this point. The characterization of the oscillation revealed, that it is not necessarily stable over the time of an experiment (i.e. $5-8$ minutes), but instead can be interrupted or can end before the experiment finishes. Therefore we have to ask the question whether the oscillation is self-sustained (i.e. the phase space representation is a limit cycle) or an intermittent type of behavior?

\footnotetext{
${ }^{6}$ The chemotactic index was calculated as described in equation 3.7.

${ }^{7}$ This case holds for both the LimE-GFP mutant in AX2 and in AX3 background.
} 
Periodic perturbation can be used to probe the cells and answer of this question. The results are summarized in figure 4.8. Subfigures show the attractor reconstruction of an autonomously oscillating cell (A-B) and for comparison an oscillating cell, subject to external driving of $\mathrm{T}=30 \mathrm{~s}(\mathrm{C}-\mathrm{D})$. The oscillation does not synchronize with the external input. The oscillation disappears when the first stimulus is applied and is replaced by the forced periodic pattern, similar to the response of the wildtype LimE-GFP oscillations (see figure 3.9). As mentioned previously, the amplitudes of the input and the output can not be compared directly (concentration and fluorescence). However, a release of only $10 \%$ (i.e. $1 \mu \mathrm{M}$ cAMP from its caged precursor) is considerably higher than the affinity $\left(K d_{1 / 2}\right)$ of $230 \mathrm{nM}^{8}$, saturating $50 \%$ of all membrane bound receptors (Caterina et al. (1995), Dormann et al. (2001)). The input function can therefore be considered to have a strong influence, which might distort or destroy any attracting pattern (see figure 4.8D). Subsequent experimental studies will need weaker perturbations of the system.
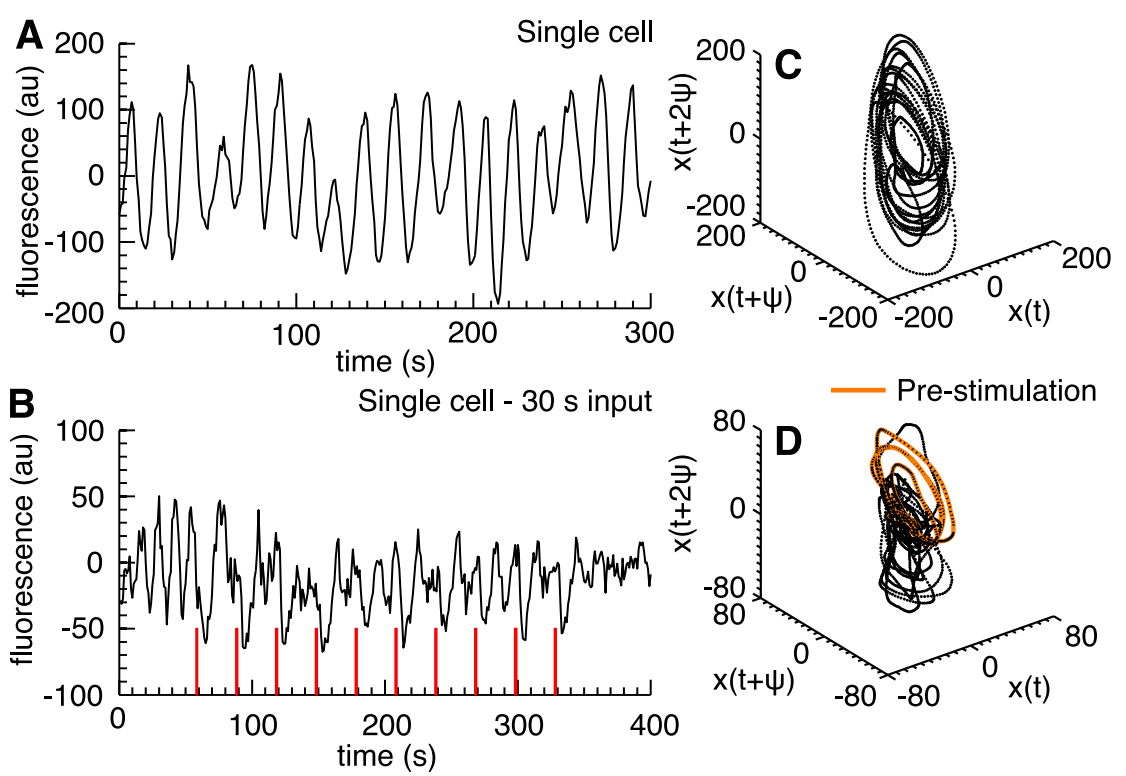

Figure 4.8.: Attractor reconstruction of an autonomous filamentous actin oscillation and an oscillation driven by an additional external forcing. The cytosolic response signal imaged (A) is time delay embedded in 3 dimensions to recover the structure of the underlying attractor (B). The same procedure is carried out for an autonomous oscillating cell exposed to periodic stimulation (C and D). The orange line marks the attractor before the stimulation starts. $\Psi$ was set to $2 \mathrm{~s}$ in both cases for optimal unfolding.

\footnotetext{
${ }^{8}$ Two affinity states of the cAR1 receptor are known, the second has an even higher affinity of $25 \mathrm{~nm}$ (Johnson et al., 1992).
} 
The pattern in figure $4.8 \mathrm{~B}$ is not restored after the end of external stimulation, unlike for the AX2 LimE-GFP wildtype, where restoration was observed (see figure 3.9). It is therefore complicated to speak of a real limit cycle oscillation, but nevertheless the argument introduced in Ishikawa-Ankerhold (2008) is still valid. In the absence of SCAR and PIR121 the actin cytoskeleton spontaneously enters repetitive cycles of polymerization and depolymerization with the system being out of balance. Here I have shown that these oscillations might arise and decay spontaneously and do not necessarily describe a stable state of the system. This indicates that the dynamics of the system are even more complex and parameters describing the system are not stationary over the duration of an experiment. 


\section{CHAPTER 5}

\section{Eukaryotic cell flattening}

Amoeboid cells and in particular the developed Dictyostelium amoeba are 3-dimensional objects, changing their shape on the timescale of a few seconds. This compromises the quality of micrographs and the duration of microscopic observations. Additionally the basal membrane of the cell bottom side, which is in close contact to the surface detaches and reattaches while the cell moves. In particular, this complicates the total internal reflection fluorescence (TIRF) microscopy, which relies on the total reflection of the laser beam on the oil glass interface (Axelrod, 1981). The exponentially decaying evanescent wave penetrates the medium up to a depth of $200 \mathrm{~nm}$. In the case of $D$. discoideum this includes the membrane and membrane associated proteins, as well as the cell cortex. To overcome these difficulties flattening or compression of the cell had been applied in order to confine the cell to one focal plane. Besides the improved image quality ${ }^{1}$ the cell is slowed down or even trapped. Traditionally overlay methods were used, i.e. the object of interest is overlayed with agar, oil or glas and consequently compressed below those. However, overlay approaches lack precise controllability of the actual cell height. This limits the reproducibility for quantitative measurements or makes those even impossible. In the sections below, we will review the overlay techniques and discuss new microfluidic approaches to this task. The review and the characterization of the devices were published as an article (Westendorf et al., 2010) and parts of the text, as well as the figures, are included in the sections 5.1-5.3. Applications and experiments, which followed this article are summarized in the final section.

$\overline{{ }^{1} \text { Confinement improves the quality of both bright field and laser micrographs. }}$ 


\subsection{Overlay techniques}

Widely used among the Dictyostelium research community is the agar overlay method (as introduced by Yumura et al. (1984)). It was applied to investigate the localization of actin and myosin in chemotactic cells (Yumura and Fukui (1985), Kitanishiyumura and Fukui (1989)) and more recently to investigate the cortical actin dynamics (Bretschneider et al. (2004), Diez et al. (2005)). In this method, the cell suspension is sandwiched between a layer of glass below and a sheet of agarose above. As fluid is removed from between these layers, the agar overlayer is lowered, and presses on the cells. A detailed description of the agar-overlay method can be found in Yumura et al. (1984) and Fukui et al. (1986). Approximately $200 \mu 1$ of a $D$. discoideum suspension in PB was placed on a cover slip $(24 \times 60 \mathrm{~mm}$, No. 1, Menzel Gläser). An agar sheet (4\% agarose, UltraPure, Invitrogen) of $c a .0 .15 \mathrm{~mm}$ thickness was placed on top of the cell suspension. Cell flattening was induced by removal of PB with filter paper (Nr. 2, Whatman International). The degree of flattening could be controlled by removing different amounts of PB.

The second flattening approach is based on an oil overlay, instead of an agar sheet. Similar to the agar overlay, the underlying principles of this method have long been established. For an early reference from the Dictyostelium literature see Potts (1902). Here, we proceeded as follows: An amount of $100-200 \mu \mathrm{l}$ of a $D$. discoideum cell suspension is placed on a glass bottom Petri dish (Matek). The dish is $50 \mathrm{~mm}$ in diameter, with a $14 \mathrm{~mm}$ circular well in the center. To maximize the hydrophilicity of the surface, we replaced the glass bottom with a fresh cover slip (No. 1, Menzel Gläser), and this modified dish was then plasma cleaned for $\sim 30 \mathrm{~s}$ (PDC 002, Harrick Plasma) immediately before depositing the cells. After waiting 15 minutes, to allow the cells to settle, we remove most of the PB with a pipette, leaving a thin wetting layer on the glass surface. A wet cotton thread is then laid along the circumference of the well. To prevent the wetting layer from drying out, we coat it with an oil layer (Neutral oil, Euro OTC Pharma), leaving one end of the thread exposed to the air outside. As this portion of the thread dries, the aqueous fluid underneath the oil layer is gently wicked away, resulting in a thinning of the wetting layer. When the desired degree of flattening is achieved, the wick is removed. The setup is illustrated in figure 5.1.

Following the protocol presented above, we flattened D. discoideum cAR1-GFP cells (Xiao et al., 1997) by an agar and an oil overlay. In the agar overlay method, the blotting of the fluid between the agar sheet and glass is difficult to control, and demands great care. Under the agar overlay it was possible to flatten the cells to less than $3 \mu \mathrm{m}$ in height (figure 5.2A and $\mathrm{C}$ ). The temporal stability of flattening is limited by the drying of the agar. To overcome this limitation, a humid environment is required, as reported in Feneberg et al. (2001). 


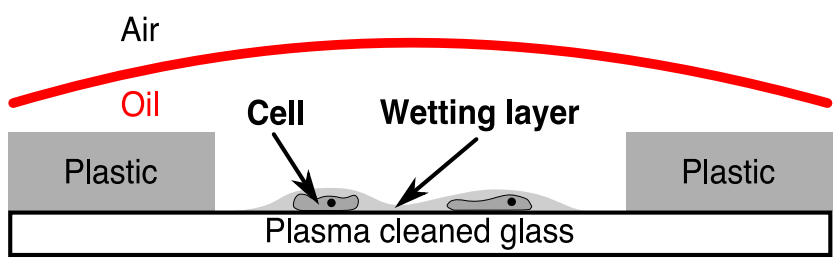

Figure 5.1.: Oil overlay technique. A drawing of the oil overlayer technique on a modified glass bottom Petri dish. The arrows mark the $D$. discoideum cells (not to scale) and the surrounding wetting layer. Most of the wetting fluid is removed by a cotton thread (not shown) placed on the glass surface, which leads to flattening of the cells.

An exemplary $D$. discoideum cAR1-GFP cell, flattened to $\sim 3 \mu$ m under an oil overlayer is shown in figure $5.2 \mathrm{~B}$ and $\mathrm{D}$. We observed that the degree of flattening was prone to strong variations. In particular, flattening was strongly dependent on the distance between the cell and the wick. Flattening was further limited by the breakup of the aqueous layer, see figure 5.7A. This break up of the wetting layer into isolated islands was frequently observed when liquid in the aqueous layer was reduced beyond a critical amount. Due to these limitations, the degree of flattening was harder to control, compared to the agar overlay.
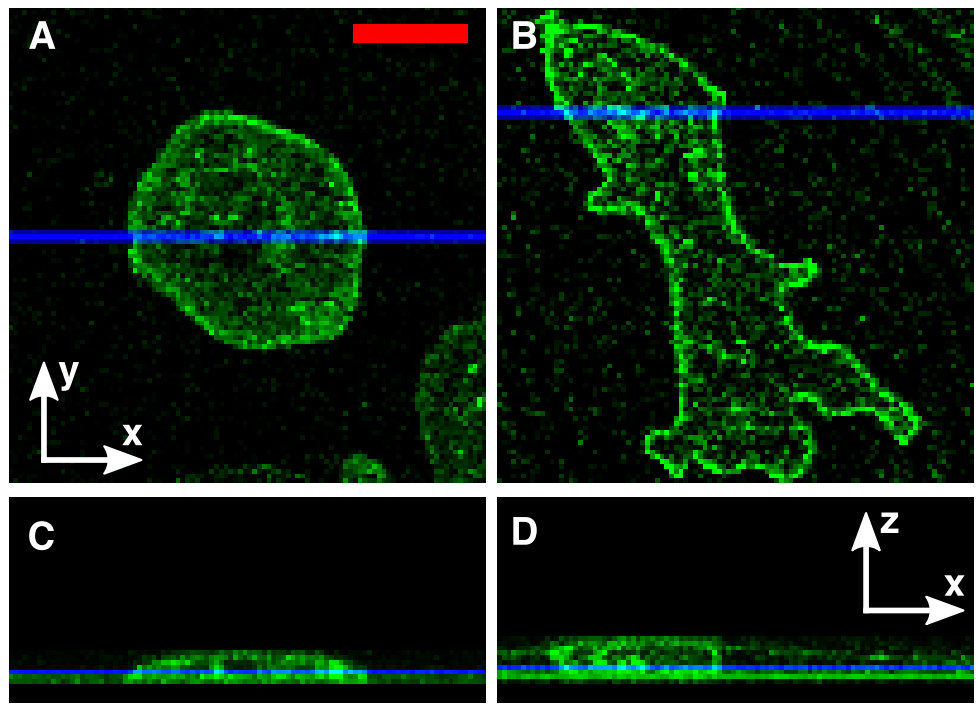

Figure 5.2.: Flattening of $D$. discoideum under agar and oil overlayer. cAR1-GFP labeled cells below an agar (A,C) and oil (B,D) overlayer. (A) and (B) show confocal x-y scans. (C) and (D) show the corresponding $\mathrm{z}$-stacks of those cells. The blue lines in $(\mathrm{A}, \mathrm{B})$ show the $y$-values of sections $(C, D)$, and the blue lines in (C,D) show the z-value of images (A,B). Scale bar: $10 \mu \mathrm{m}$.

We used the agar overlay technique as the reference to compare the stability, the degree 
of control, and the ease of operation for the microfluidic cell flattening techniques. The protocol is well known and tested. It has been used, for example, to apply mechanical stress to living cells (Clarke and Maddera, 2006) or to enhance live cell imaging by deforming cells into a quasi two-dimensional geometry (Neujahr et al., 1998). Also, agar overlay experiments have been performed where liquids have been added and removed during flattening (Fukui et al., 1986). However, the removal and reintroduction of fluid underneath the agar overlay is difficult to perform and hard to control quantitatively, leaving a high risk of damaging the cells. Finally, even cover glass can be used as overlay, which led to the development of the rotocompressors (see Aufderheide (2008) for a review). These have not been treated in the published article, but should be mentioned here for the sake of completeness. The rotocompressors rely on precisely bending a coverglass, squeezing a specimen underneath. The different rotocompression chambers improve the image quality by flattening, but were initially designed to trap fast moving microorganisms like protozoa (Spoon (1978), Uhlig and Heimberg (1981), Aufderheide (1986)). Contrary to the traditional overlay techniques the rotocompressors allow well controlled repeated cycles of compression and decompression. As we will see in the following section the microfluidic chambers, established within this work, also include this advantage.

\subsection{Microfluidic approaches}

\subsubsection{Single layer microfluidic channel}

The single layer microfluidic device ${ }^{2}$ consists of a single PDMS layer with a simple throughflow micro-channel, see figure $5.3 \mathrm{~A}^{3}$. The key feature of this channel is the high aspect ratio (width $\gg$ height) chamber in the middle part. A syringe pump is used to withdraw liquid through the channel. The higher the flow rate, the greater the pressure drop between the outer atmosphere and the chamber. This pressure difference lowers the elastic PDMS ceiling, causing the cells below to be flattened. figure 5.3B shows the layout of the chrome mask used to fabricate the single layer microfluidic device. The final height of the microfluidic channel after spincoating the SU8-25 photoresist was $14 \mu \mathrm{m}$ (the taller the microfluidic channel, the higher the flow speed required to flatten cells inside the channel). The area of the chamber, where experiments were carried out was $2 \times 2 \mathrm{~mm}$ in size. It was stabilized by two rows of pillars (figure $5.3 \mathrm{~B}$, in red), to prevent uncontrolled collapsing. We used

\footnotetext{
${ }^{2}$ The development and the characterization of the microfluidic flattening devices was carried out together with Albert J. Bae and Christoph Erlenkämper.

${ }^{3}$ Original idea of the single channel flattening chamber by Carl Franck.
} 
$45 \mathrm{~g}$ of PDMS and $4.5 \mathrm{~g}$ of curing agent (100:10), to create a $c a .3 \mathrm{~mm}$ tall microfluidic device. After the device was assembled, it was heated at $75^{\circ} \mathrm{C}$ for 10 minutes to prevent an untimely, irreversible collapse of the chamber ceiling. The cells were loaded as described above. Then, the outlet was connected to a reservoir of PB (see figure 5.3C). To lower the PDMS ceiling, PB was withdrawn from the channel at a constant rate using a syringe pump (PHD 2000, Harvard Apparatus). The degree of flattening depends on the suction rate. It is crucial to remove bubbles from the microchannel, the tubing, and the syringes, since they may expand in volume, leading to an uncontrolled drop in the actual flow speed in the microchannel.

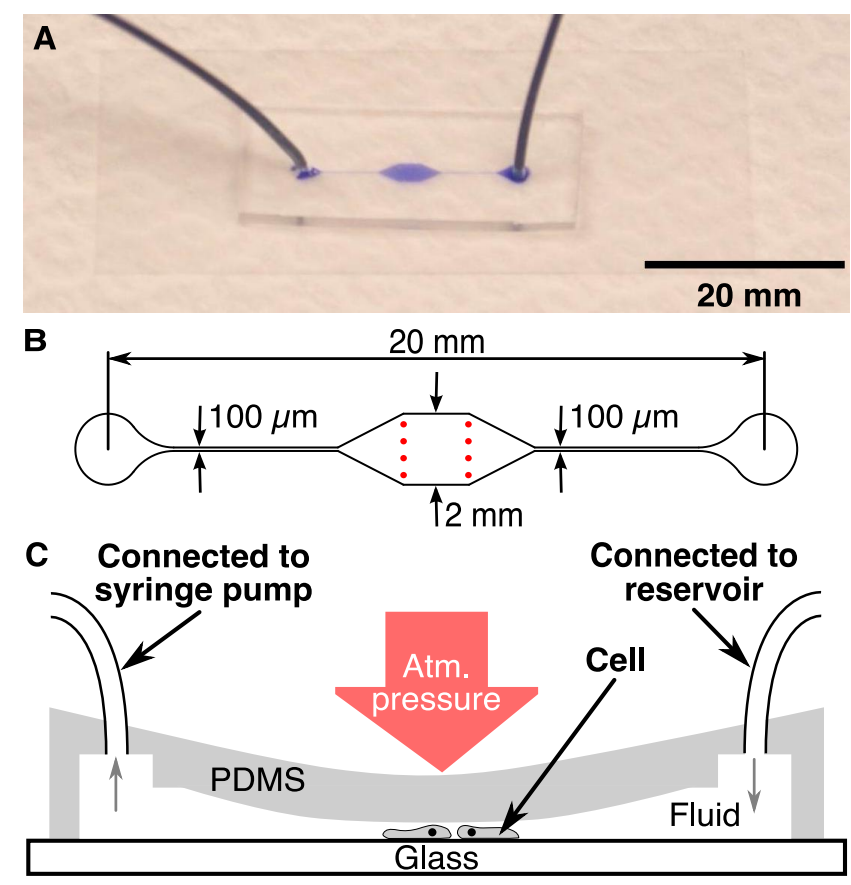

Figure 5.3.: Single layer microfluidic device. (A) Assembled single layer device with connected tubing, filled with ink. (B) Geometry of the chrome mask used to create the single layer device. The height of this channel was $14 \mu \mathrm{m}$. Two rows of pillars (red spots) support the ceiling. In (C), the principle of operation is shown. The inlet of the collapsing device is connected to a reservoir, filled with PB. A syringe pump removes the fluid at a constant rate (indicated by the gray arrows) and thus decreases the pressure within the microfluidic channel. This leads to a collapse of the ceiling and therefore to flattening of $D$. discoideum cells located in the channel. Sketch is not to scale.

By adjusting the rate at which fluid is withdrawn from the single layer device, we can tune the degree of flattening. Then, when we fix the flow rate, the channel height will eventually approach a constant value, which in turn, allows us to conduct stable, long term microscopy on flattened cells. The device characterization with fluorescein is shown in figure 5.4A. 
Here, we applied a suction speed of $250 \mu \mathrm{l} / \mathrm{h}$, which reduced the channel height by $67 \%$ (from 14 to approximately $4.7 \mu \mathrm{m}$ ). After the fluid flow was stopped, the ceiling relaxed back into its initial position. During the first 5 minutes, $80 \%$ of the original height was recovered, but it took more than 16 minutes to relax back into its original height. The withdrawal speed of $250 \mu \mathrm{l} / \mathrm{h}$ was also used to flatten cAR1-GFP cells. The confocal $\mathrm{x}-\mathrm{y}$ scans of a cell before flattening, and during flattening are shown in figure $5.4 \mathrm{~B}$ and $\mathrm{C}$. The height of the cell decreased from $11 \mu \mathrm{m}$ (figure 5.4D) to $\sim 4 \mu \mathrm{m}$ (figure 5.4E) in this device.
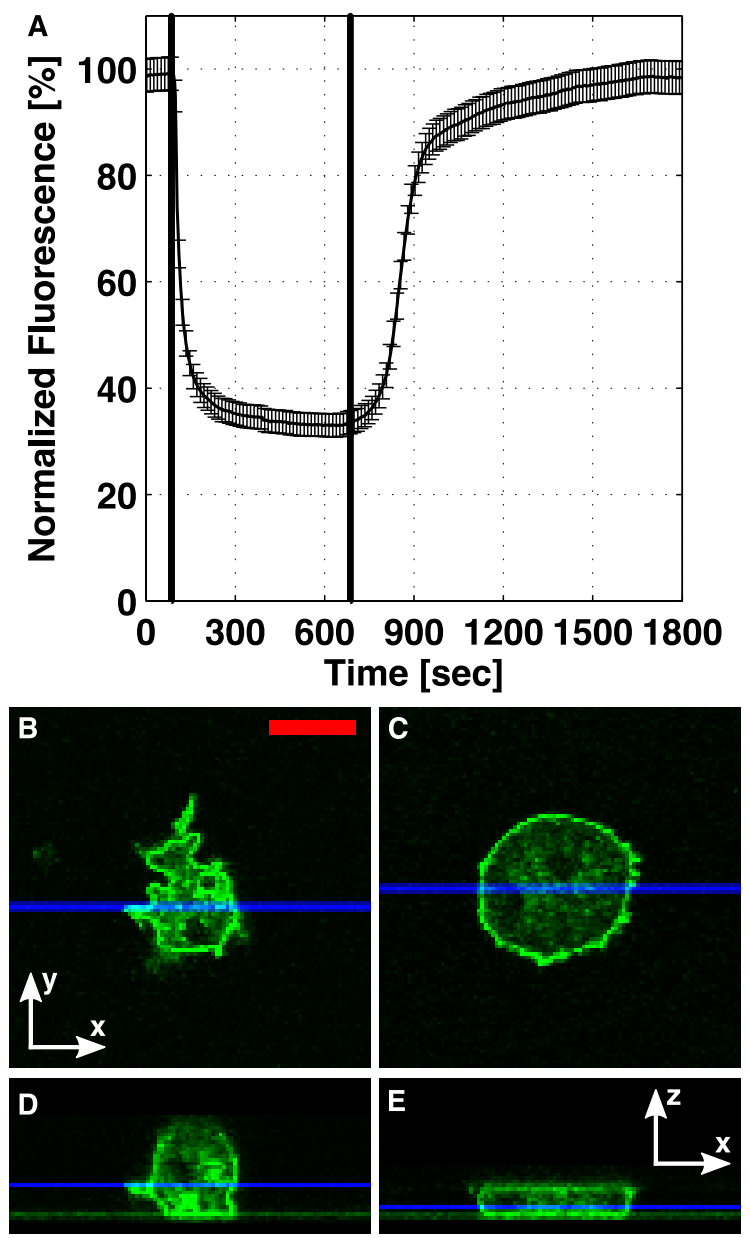

Figure 5.4.: Characterization of the single layer microfluidic device. (A) The channel was filled with $100 \mu \mathrm{M}$ fluorescein, and the intensity in the middle of the device was recorded. Between $\mathrm{t}=$ $85 \mathrm{~s}$ (line 1 ) and $\mathrm{t}=685 \mathrm{~s}$ (line 2), the syringe pump withdrew $250 \mu \mathrm{l} / \mathrm{h}$ of the fluorescein through the device, leading to a drop in the channel height. This flow rate was used to flatten cAR1-GFP cells. Confocal $x-y$ scans are shown for a cAR1-GFP cell (B) before and (C) during flattening. (D,E) show the corresponding $\mathrm{X}-\mathrm{Z}$ sections. The blue lines in $(\mathrm{B}, \mathrm{C})$ show the $\mathrm{y}$-values for the sections $(\mathrm{D}, \mathrm{E})$, and the blue lines in $(\mathrm{D}, \mathrm{E})$ show the $\mathrm{z}$-value of images $(\mathrm{B}, \mathrm{C})$. Scale bar: $10 \mu \mathrm{m}$. 


\subsubsection{Microfluidics through-flow actuator}

The through-flow actuator is composed of two layers, an actuation channel on top and a channel for cell flattening below. The lower channel is filled with a $D$. discoideum cell suspension or a fluorescein solution for characterization. In contrast to the previous setup, the actuation channel is a through-flow channel connected to a continuous oxygen supply. The flow of oxygen is controlled by a metering valve. When the pressure in the actuation channel is increased, the PDMS sheet between the two layers is deformed, flattening the cells (see figure 5.5B). The microstructures were prepared by photolithography on two different "master wafers." The layout of the first wafer, used for molding the actuation channel, was the same as for the single layer device (see figure 5.3B) with a height of $28 \mu \mathrm{m}$. The actuation channel was made by covering the wafer with a layer of the PDMS (100:10) about $1 \mathrm{~cm}$ thick. To prepare the thin lower layer, we used a second wafer with a channel structure of $500 \mu \mathrm{m}$ width and $24 \mu \mathrm{m}$ height (figure 5.5A). In order to distribute the PDMS uniformly, two spacers were used, each consisting of three cover slips (No. 1) that had been glued together. The spacers were taped to opposing edges of the wafer and a bubble-free PDMS (100:10) droplet was placed between them.

The long edge of a thick glass slide was used to evenly spread the PDMS between the two spacers. Before curing the PDMS, the spacers were removed. The actuation channel was cut out and holes were punched into the PDMS. After plasma cleaning, this PDMS block was bonded on top of the thin PDMS film on the master wafer, containing the lower channels. Then, the thin layer was cut along the edges of the PDMS block and the two bonded layers were carefully removed from the wafer. After this, holes for the lower channel were punched into the PDMS. The structured side of the device and a cover slip were plasma cleaned and bonded together as described. The assembled microfluidic through-flow actuator is displayed in figure 5.5C. A suspension of D. discoideum LimE-GFP cells was filled into the lower channel. After 15 minutes, PB was supplied with a constant flow rate of $5 \mu \mathrm{l} / \mathrm{h}$ for the entire experiment. The pressure in the actuation channel was controlled by a metering valve, located between the channel inlet and the oxygen supply ( 0 to 1 bar above atmosphere) as shown in figure 5.5D. The pressure was measured with a glycerin filled manometer (0 to 1 bar, Swagelok). 

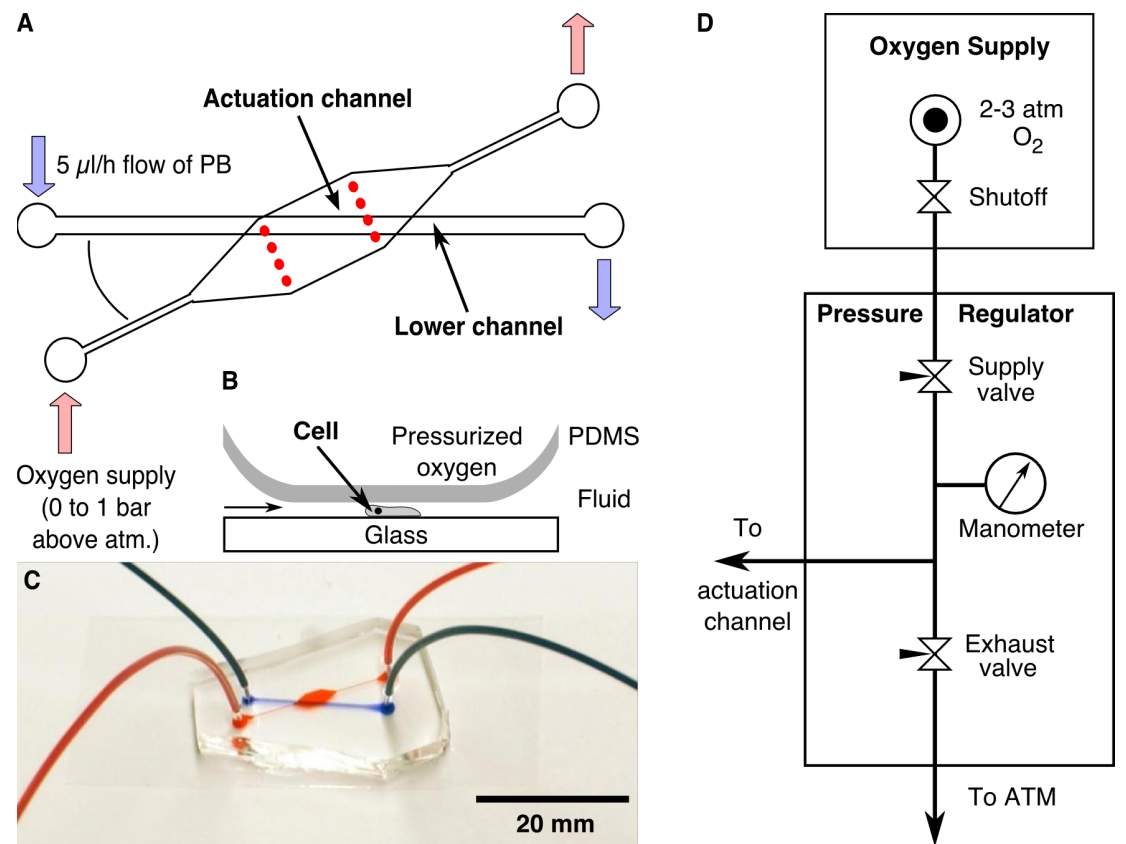

Figure 5.5.: Microfluidic through-flow actuator. (A) Similar to the closed-end actuator, the cells are contained in the bottom channel. In this case, however, the actuation channel was open ended allowing oxygen to flow through. The oxygen was supplied to the cells via the permeable PDMS membrane which separates the two layers (B). By adjusting the pressure of the oxygen supply at the inlet, this PDMS membrane was deformed and flattened the cells. The angle between both channels was limited by the size of the cover slip. (C) Assembled double layer microfluidic channel with connected tubing. The actuation channel is filled with red and the lower channel with blue dye. (D) Schematic of the pressure regulation of the oxygen flowing through the actuation channel. The manometer measures the pressure applied to the actuation channel.

We tune the height of the cell-containing channel by adjusting the pressure in the actuation channel above. The through-flow actuator was characterized in figure 5.6. In these figures, subfigure A shows the temporal evolution of the lower channel height when flattened and unflattened. Subfigure B shows height profiles across the channel, for different degrees of flattening. Subfigures C - F show confocal images for a cAR1-GFP cell before (C,E), and during $(\mathrm{D}, \mathrm{F})$ flattening. By quickly adjusting the oxygen pressure in the actuation channel, we were able to change the channel height from $24 \mu \mathrm{m}$ to less than $1 \mu \mathrm{m}$ in less than $10 \mathrm{~s}$ (figure 5.6A). The reverse process had slightly longer time resolution, $\sim 20 \mathrm{~s}^{4}$. The limitations in speed and stability of this device are caused by the pressure supply. Pressure is applied by manually opening a needle valve on the supply end, and is released by closing this valve and opening an exhaust needle valve (figure 5.5D). Replacing this system with a more sophisticated automated pressure control should improve the stability of the flattened

\footnotetext{
${ }^{4}$ The corresponding movie can be found in the electronic supplement.
} 
state, and the speed at which flattening/unflattening occurs. In the first variant of this double layer device, the actuator is a closed-end channel, i.e. the actuation channel has only an inlet but no outlet and is filled with water. The initial closed-end actuator, created by Edouard Galland, can be found in the published article (Westendorf et al., 2010). The rates of pressurization and de-pressurization in the actuation channel are governed by the speeds at which the connected syringe pump runs. By running the syringe pump at the same rate, in reverse, the lower channel is unflattened in about the same amount of time it took to flatten, which is an advantage over the through-flow approach.
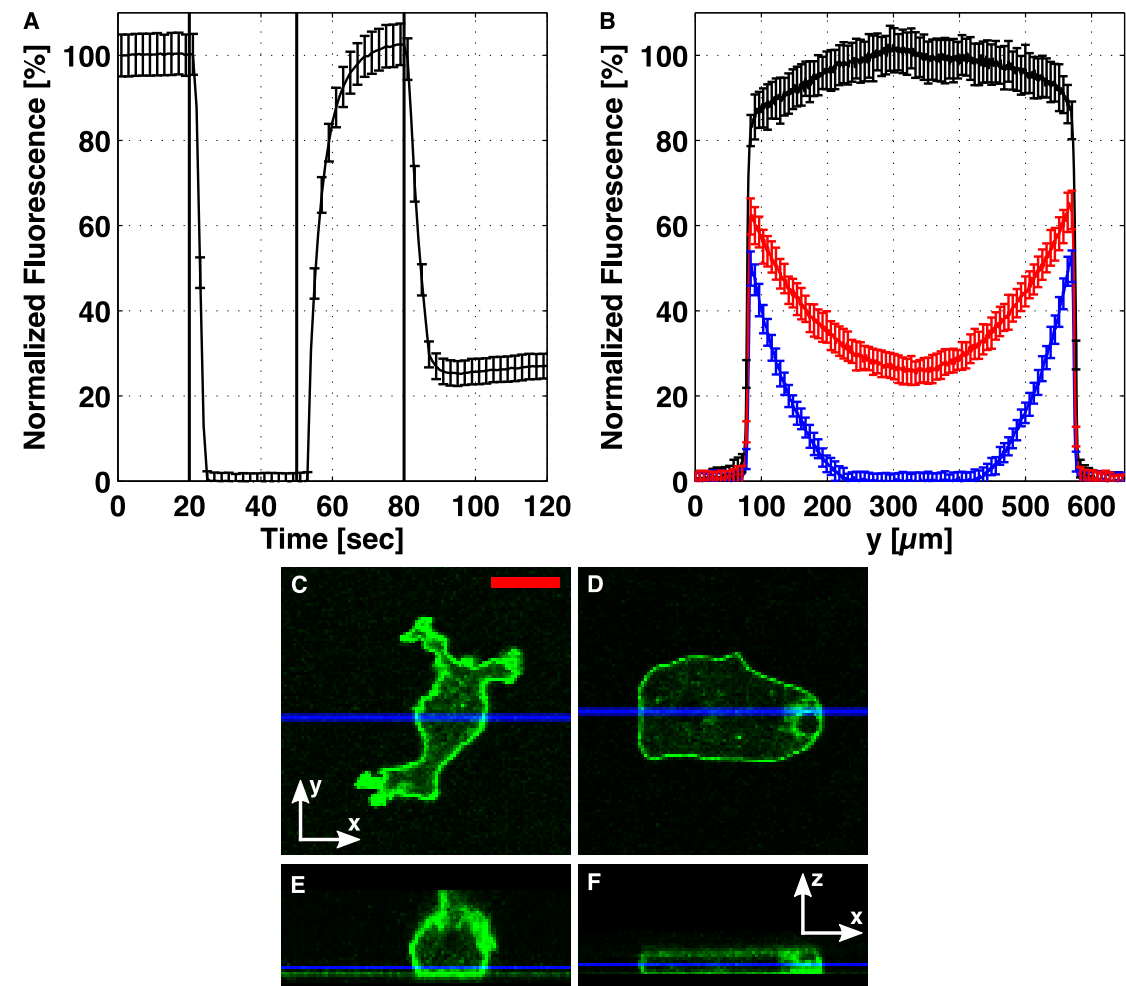

Figure 5.6.: Characterization of the through-flow actuator. The lower channel was filled with $100 \mu \mathrm{M}$ fluorescein, and in (A), the fluorescence in the middle of the channel is shown. At $\mathrm{t}=20 \mathrm{~s}$ (line 1), the pressure in the actuation channel was increased by 0.4 bar, at $t=40 \mathrm{~s}$ (line 2 ), the pressure was released, and at $\mathrm{t}=80 \mathrm{~s}$ (line 3 ), the pressure was increased to 0.2 bar above atmosphere. (B) shows the profile across the channel for different degrees of flattening, with the black, blue, and red curves corresponding to $\mathrm{t}=15 \mathrm{~s}, 40 \mathrm{~s}$, and $105 \mathrm{~s}$ in (A). Confocal $\mathrm{x}-\mathrm{y}$ scans are shown for a cAR1-GFP cell (C) before and (D) during flattening. (E,F) show the corresponding $\mathrm{X}-\mathrm{Z}$ sections. The blue lines in (C,D) show the $y$-values for the sections (E,F), and the blue lines in (E,F) show the z-value of images (C,D). Scale bar: $10 \mu \mathrm{m}$. 


\subsection{Discussion}

We have presented classical overlay techniques and more elaborate microfluidic methods for flattening eukaryotic cells. Overlay techniques achieve cell flattening by the removal of the fluid between the glas floor and a confining top layer. The microfluidic approaches rely on applying a pressure difference across a deformable PDMS layer, which acts as a ceiling for the cell flattening chamber.

The height of this PDMS ceiling is adjusted by changing this pressure difference. Variations of the oil overlay method have been used, for example, for the observation of quasi two-dimensional slugs of D. discoideum during its developmental cycle (Bonner, 1998), as well as to investigate meiosis in insect spermatocytes (Fleming and Rieder, 2003). The oil overlay technique is harder to control than the agar overlay method. The height of the aqueous layer is dependent on the distance from the wick and suffers from variations due to the breakup of the wetting layer. Furthermore, controlled exchange of the aqueous medium is even more difficult to achieve than in the case of the agar overlay once the cells are confined underneath the oil layer. The oil overlay method also has a number of advantages compared to the agar overlay. For instance, if stable operating conditions are required, the oil overlay is preferable to the agar assay since the latter requires a humid environment to prevent it from drying. For a brief review of overlay techniques see also Fleming and Rieder (2003). To complement the overlay techniques, we have developed single and double layer microfluidic devices to perform well-controlled flattening of living cells. The single layer device is the easiest to fabricate. The high aspect ratio, which is necessary for the operation of this device, allows many cells to be flattened at once. The relaxation time of this device, after flattening, is greater than $15 \mathrm{~min}$. Therefore, this device has only limited applicability for repeated cycles of flattening and relaxing. Furthermore, it is often desirable to decouple the control of flattening from the fluid flow in the channel containing the cells.

To accomplish this, an actuation channel is added above the cell flattening channel. The actuation channel can either be open, i.e. a through flow channel with an inlet and outlet, or closed with only an inlet. A closed-end device is well-suited for long term microscopic imaging experiments, because no continuous flow in the actuation channel is necessary. On the other hand, the through-flow device allows the continuous delivery of oxygen to the cells through the permeable PDMS film between the upper and lower layers. When the pressure is released, these devices recover to their initial states within a minute (see figure 5.6A). Note that the use of spacers to control the thickness of the PDMS film between the actuation and the compression channel makes our protocol less demanding in terms of equipment compared to the PDMS spin-coating approach that is commonly used for two- 
layer microfluidic fabrication (Squires and Quake (2005), Kim et al. (2007)). The main advantage of microfluidic methods compared to overlay techniques is the high level of control. Flattening can be tuned and ranges from gentle cell compression, to immobilization by mechanical trapping, and destruction by bursting the cell membrane. Due to the continuous flow, the chemical environment of the cells can be much better controlled inside a microfluidic flattening device. On the one hand, the medium surrounding the cells is continuously exchanged, so that waste products and signaling substances released by the cells themselves are removed and a stationary chemical environment can be implemented. On the other hand, additional chemical factors may be added to the fluid flow and even modulated in the course of the experiment, so that flattening and chemical stimulation can be realized simultaneously. Note that additional valves may be required to add chemical species to the fluid flow upstream of the actual flattening chamber.
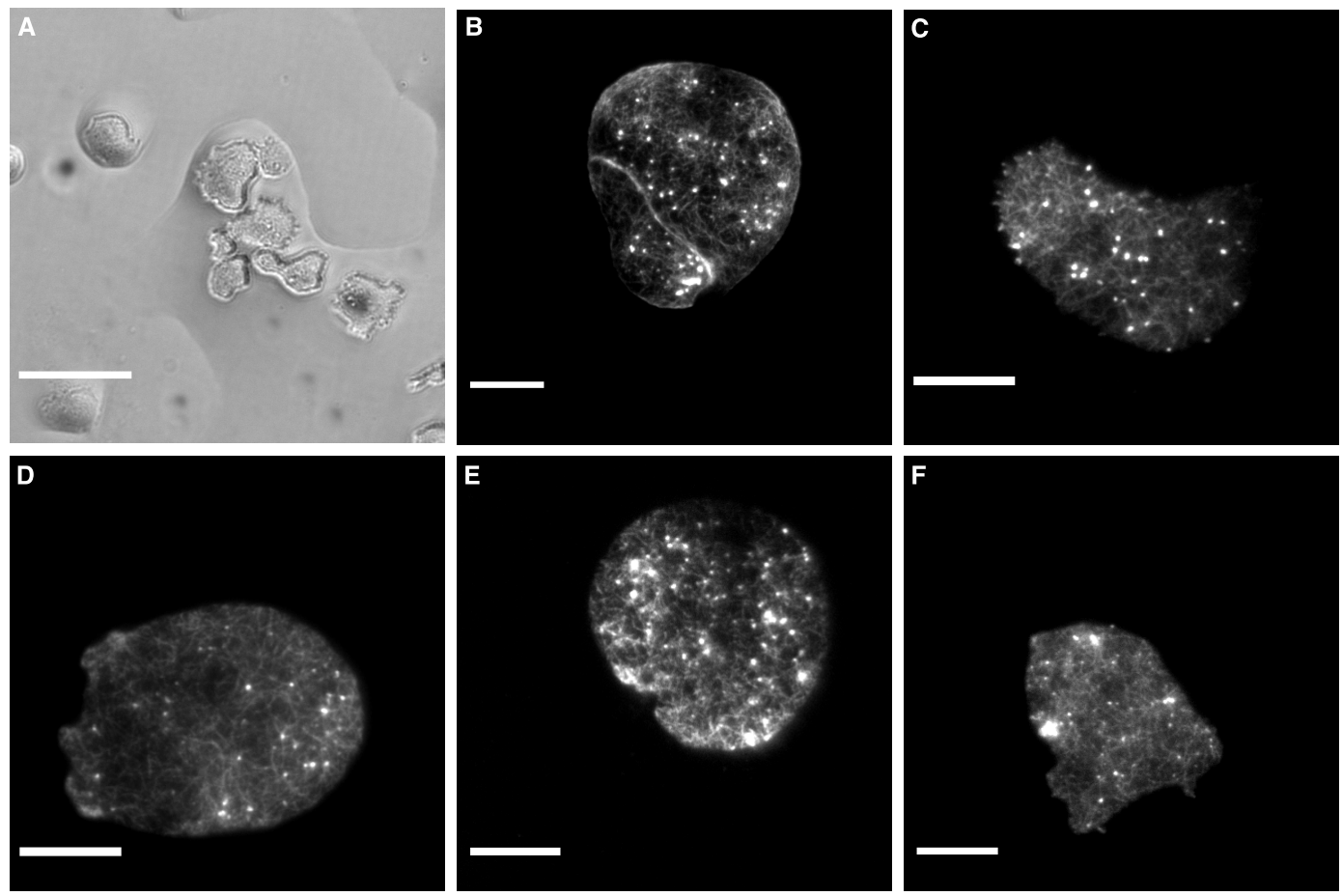

Figure 5.7.: Sample images using D. discoideum LimE-GFP. (A) BF image of cells under an oil overlayer. The breakup of the wetting layer can be seen, with flatten cells confined inside small aqueous islands. (B-F) TIRF microscopy images of cells flattened by the agar overlay (B), the oil overlay (C), the single layer microfluidic device (D), the closed-end actuator (E) and the throughflow actuator (F). Scale bar in A is $50 \mu \mathrm{m}$ and the scale bars in B to E display $10 \mu \mathrm{m}$. 
Despite these advantages, microfluidic techniques also have a number of drawbacks. Due to the rigidity of PDMS, the ceiling of the microfluidic flattening chambers will not adjust to the shape of the cells. In contrast, the overlay techniques will adapt to some extent to the morphology of the cells (figure 5.2C and D). This can be advantageous if, for example, large mechanical stress on the nucleus has to be avoided. Besides, the manufacturing of microfluidic devices requires elaborate micro-fabrication techniques like photo-lithography and polymer molding. Consequently, the assembly of microfluidic devices is more time consuming than the overlay techniques. Usually it takes several days to setup a microfluidic device, beginning with the chrome mask generation. The operation of microfluidic setups is also considerably more expensive, since advanced tools like micro-liter syringes and precision pumps are required.

Great care has to be taken to avoid leakage or inclusions of air in the tubing or the microchamber. Such gas inclusions would obstruct the stable operation of a microfluidic devices because of their large compressibility. This is especially true for the single layer microfluidic device, which achieves flattening by withdrawing the liquid from the channel. For imaging experiments, which do not require the application of drugs or a constant supply of nutrients, the agar overlay method is the most reliable. If a continuous fluid flow is needed, or direct and precise control of flattening is desired, then the microfluidic based flattening devices are superior to the overlay techniques. Among the microfluidic cell flattening devices described here, the through-flow actuator combines the advantages of high controllability, medium construction time, and reproducibility. To conclude, we summarize the key advantages and disadvantages of the five setups in table 5.1.

\begin{tabular}{cccc} 
Name & $\begin{array}{c}\text { Control of } \\
\text { flattening }\end{array}$ & $\begin{array}{c}\text { Control of } \\
\text { environment }\end{array}$ & $\begin{array}{c}\text { Construction } \\
\text { time }\end{array}$ \\
\hline \hline Agar overlay & no direct control $^{1}$ & no & $<1$ hour \\
Oil overlay & no direct control $^{1}$ & no & $<1$ hour \\
Single layer & via syringe pump $^{2}$ & yes $^{2}$ & $2-3$ hours $^{3}$ \\
Closed-end actuator $^{4}$ & via actuation channel $^{3}$ & yes (lower channel) & $>3$ hours $^{3}$ \\
Through-flow actuator $^{3}$ & via actuation channel & yes (lower channel) & $2-3$ hours $^{3}$ \\
\hline
\end{tabular}

Table 5.1.: Comparison of devices for eukaryotic cell flattening. Remarks: ${ }^{1}$ Indirect control via the removal of liquid below the overlayer. ${ }^{2}$ The syringe pump by which the collapse of the ceiling is controlled, might also be used to suck in nutrients or drugs. ${ }^{3}$ The time estimate takes only the assembling of microfluidic channels into account. The chrome mask generation, patterning of the $\mathrm{Si}$-wafer, and the following molding of the PDMS take more than a day. ${ }^{4}$ The characterization of the closed end actuator can be found in Westendorf et al. (2010). 


\subsection{Applications of the cell flattening devices}

Now we shall draw our attention on applications of the microfluidic devices, described above. Albert Bae showed two principal applications of the double layer flattening technique, which I will briefly review here (see Bae (2010) for more details):

- The double layer device was used to flatten entire cell aggregates to a cell layer of single cell thickness. This simplifies tracking of the cells while they spin around the aggregation center. This task was approached previously, but the thickness of the aggregate was still several layers of cells (Bonner (1998), Nicol et al. (1999)) or the cells have been plated as monolayers initially but the movement was not restricted in z-direction (Ma et al. (1997), Chien et al. (2000)). Here the microfluidic channel gives the necessary control over the aggregate height and additionally the aggregate is supplied with oxygen via the actuation channel (The importance of oxygen for the Dictyostelium development was investigated in Bonner et al. (1998)).

- D. discoideum moves too fast in order to obtain the exact three-dimensional cell shape. The confinement to almost two dimensions allows the quick readout of the cell geometry and the acquired stack of geometries was successfully used to simulate the fluid flow around a Dictyostelium amoeba. Therefore an initially 3 dimensional question on the swimming of Dictyostelium discoideum in dense fluids (see Barry and Bretscher (2010)) was reduced to two dimensions using microfluidic flattening (Bae and Bodenschatz, 2010).

Skoge et al. (2010) published a low ceiling device, which in principle would also allow to investigate the aggregation of $D$. discoideum in 2 dimensions. The height of the ceiling was set to $2 \mu \mathrm{m}$ and therefore considerably lower than the lowest heights observed within this work, since all cells flattened below height of approx. $3 \mu \mathrm{m}$ were destroyed ${ }^{5}$. A fixed ceiling height therefore might act as a selector, only allowing a fraction of cells to enter it. However, the authors stated that they did not observe a selection of the amoebae by the low ceiling device (Rappel, 2010).

I focused on the cortical actin dynamics imaged with TIRF-microscopy. This task was first approached by Diez et al. (2005) using the agar overlay method. Here I extended this analysis of a flattened LimE-GFP cell by the automated detection of the LimE-GFP foci. These temporary structures are regions of high filamentous concentration (see figure 5.8A, bright spots in the cell cortex). It has been hypothesized that they mediate the cell substrate ad-

\footnotetext{
${ }^{5}$ The membrane ruptured under this degree of flattening, which resulted in the release of the cytosol and the organelles.
} 
hesion $^{6}$ (Uchida and Yumura, 2004). In figure 5.8 a LimE-GFP cell is flattened using the single layer microfluidic flattening device. The $\mathrm{area}^{7}$ is close to constant (see figure 5.8D), although the cell is still able to protrude pseudopods. Therefore the microfluidic flattening is stable enough to identify and track the actin foci over time. The actin foci are detected after bandpass filtering the image and subsequent binary thresholding. The detected spots are shown as blue circles in figure 5.8B. The total number of detected actin foci over time is given in figure 5.8C. It is decreasing and shows strong fluctuations, which have been reported previously (Uchida and Yumura, 2004). The area of the cell attached to the glass surface is almost constant over the course of the experiment (see figure 5.8D). This shows again the stability of microfluidic cell flattening.

Standard beadtracking algorithms are sufficient for tracking the detected actin foci over time. The distribution of persistence times (i.e. the length of the tracks) is given in figure $5.8 \mathrm{E}$. Due to the automated process the peak at short lengths is introduced artificially ${ }^{8}$. The second maximum is between persistence times of 10 to $20 \mathrm{~s}$. This is in agreement with Uchida and Yumura (2004), where a turnover time of $15 \mathrm{~s}$ was observed. Due to the flattening technique we are now able to trace the adhesion points in a very slow moving cell. In figure $5.8 \mathrm{~F}$ the outlines of the cell show its movement. The traces are treated as simple lines between the first (appearance of the spot) and the last (vanishing of the spot) time point (figure 5.8F). The angle of the track with respect to the $\mathrm{x}$-axis is computed and its value color-coded (figure $5.8 \mathrm{G}$ shows the color coding). It becomes clear that the actin foci in the rear of this slow moving cell show a preferred direction.

However, it is still debated, whether these actin foci mediate the substrate adhesion (as hypothesized by Uchida and Yumura (2004)). Paxilin, which is a key regulatory element of focal adhesions (Giannone et al. (2004), Webb et al. (2004)) was also found in D. discoideum, but does not co-localize with the observed foci (Bukahrova et al., 2005). The results of this group are contradictory to Uchida and Yumura (2004) and this issue has not resolved yet. The actin foci might also indicate the localization of the filamentous actin with micropinosomes ${ }^{9}$. Clathrin, which coates these vesicles, is known to induce F-actin polymerization close to the internalized vesicle (Clarke and Maddera, 2006).

\footnotetext{
${ }^{6}$ Integrins have not been detected in Dictyostelium discoideum and therefore the mediator between the extracellular matrix and the actin foci on the membrane inside remains unknown.

${ }^{7}$ Area was calculated by adaptive thresholding to compensate the cells loss of fluorescence intensity over time.

${ }^{8}$ The algorithm counts detections, which result from noise, as short tracks and hence a large artificial peak at short track lengths is produced.

${ }^{9}$ Micropinosomes are membrane inclusions formed by endocytosis.
} 

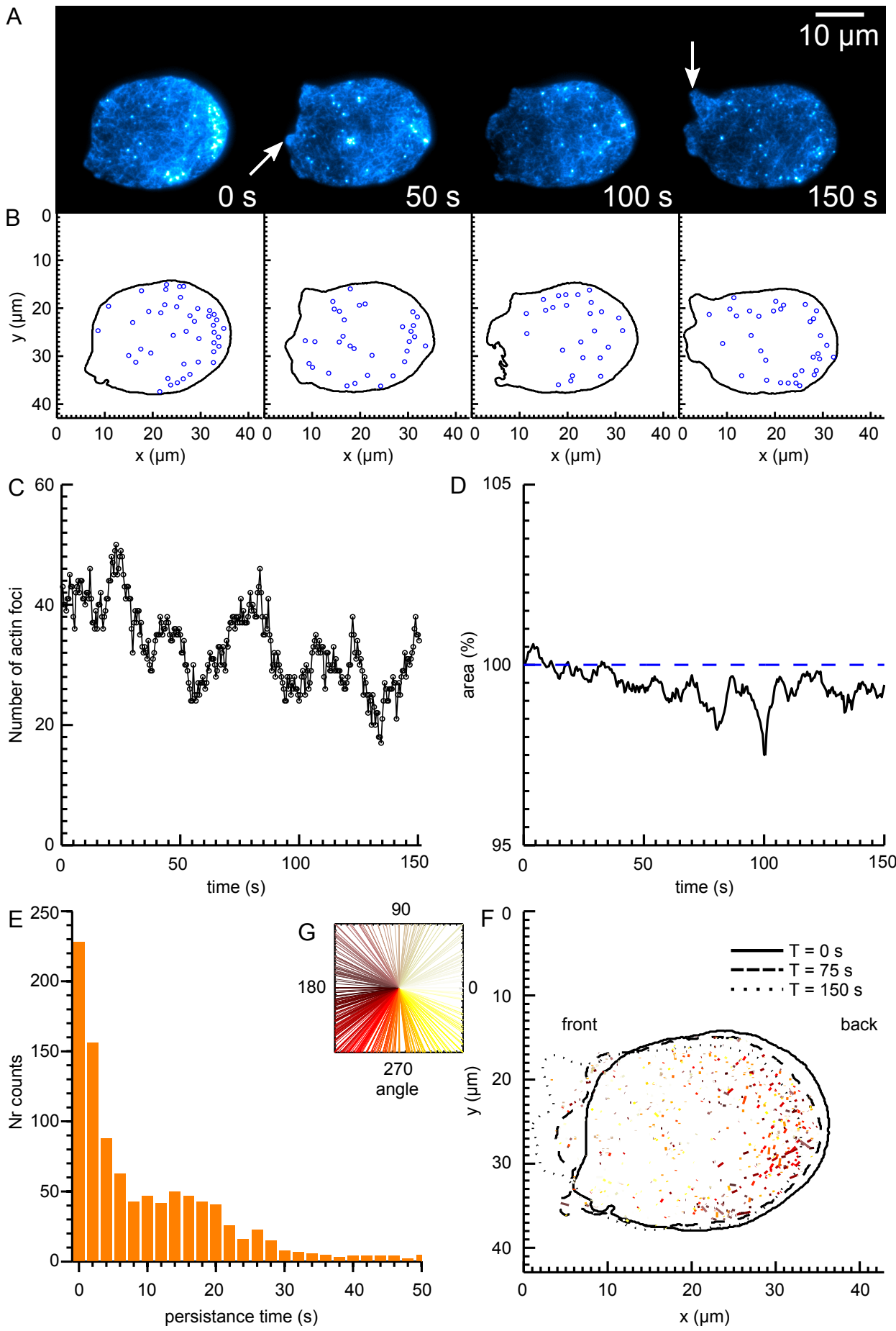

Figure 5.8.: Example analysis of a flattened Dictyostelium cell. (A) Micrographs of the cell (flattened in a single layer device) taken with TIRF-microscopy. The respective movie can be found in the electronic supplement. Gray values are colored in cyan for visibility. (B) shows the detected actin foci in blue. Detection applied bandpass filtering and binary thresholding. The number of foci over time is given in (C). The area over time under flattening is in this case close to constant. The cell however is still able to protrude pseudopods (see $(\mathrm{A}) \mathrm{t}=50 \mathrm{~s}$ and $\mathrm{t}=150 \mathrm{~s}$ ). A beadtracking routine was applied to track the actin foci. The length of each track gives the persistence time of the adhesion point. The distribution of persistence times is plotted as a histogram in (E). Due to the automated detection process, the noise (track lengths $\leq 5 \mathrm{~s}$ ) is high, but the second maximum between $10 \mathrm{~s}$ and $20 \mathrm{~s}$ is visible. Beadtracking was used to reveal the movement of each foci and its direction. Each track is displayed by a line connecting the first and the last point. The angle is color-coded $(\mathrm{G})$. In the rear of the cell the actin foci have a preferred direction. 
The actin cytoskeleton allows chemotactic amoebae to rapidly respond to changing environmental circumstances. In the case of developed Dictyostelium discoideum this is the spatio-temporal location of the chemoattractant cAMP. A single short-time pulse of cAMP induces an actin cytoskeletal response, reminiscent of a damped oscillation. Within this work I have shown that the characteristics of this oscillatory response display a cell to cell variety, leaving the majority of cells with a strongly damped response. Less frequently weakly damped responses, as well as self-sustained oscillating cells have been observed. Subsequent characterization of the actin cytoskeletal oscillatory dynamics with periodic pulse trains revealed a resonance of the system to input periods equal to $20 \mathrm{~s}$.

These observations led us to conjecture that the actin cytoskeleton of chemotactic amoebae operates close to the onset of an oscillatory instability (Hopf bifurcation) (Westendorf et al., 2012). An empiric delay differential equation approach was applied to simulate the obtained data. It recovers the majority of observed temporal patterns and explains the cell to cell variability, as every cell might be closer or further away from the instability. Furthermore it can be shown that the delay $\tau$ and the period of the self-sustained oscillation are related by: $\mathrm{T}=4 \tau$ (Murray, 2003). Hence $\mathrm{T} \approx 20 \mathrm{~s}$ for the experimentally determined $\tau$. This is in excellent agreement with the observed resonance at $\mathrm{T}=20 \mathrm{~s}$.

Our model of the actin cytoskeleton operating close to the onset of an oscillatory instability also gives a possible explanation for the observed temporal patterns in the SCAR(-)/ PIR121(-) LimE-GFP mutant, as the full population of cells seems to be shifted with respect to the critical parameter, such that a larger fraction of the total cell population is beyond the instability. Hence the self sustained oscillation is observed more often, than in the wildtype. Why is the operation close to an instability advantageous for the cell? The importance of 
operating close to an instability has been frequently discussed for different biological systems (Moreau and Sontag, 2003) and in the case of the cochlea it has been suggested that its maintenance on the edge of a Hopf bifurcation, allows selective amplification of signals close to the intrinsic frequency (Camalet et al., 2000). The periods of the cAMP pulses, applied within this work, are considerably shorter than the periods of cAMP waves $D$. discoideum experiences over the course of its aggregation (timescale of minutes). However, the pseudopod formation in a migrating $D$. discoideum cell is on the same order as the observed resonance (Bosgraaf and Van Haastert, 2009). This may indicate that internal signals are selectively amplified by the cytoskeletal machinery, resulting in pseudopod formation at timescales between $10 \mathrm{~s}$ and $20 \mathrm{~s}$ (Westendorf et al., 2012). 
First of all I'd like to thank my advisor Eberhard Bodenschatz for offering me the possibility to graduate in between the lines of biology and physics. His enthusiasm about physics and physical as well as biological questions has greatly influenced me. Second I would like to thank Carsten Beta, my second supervisor, for his continuously good support over the past 5 years. Oliver Steinbock agreed to be the second referee of my $\mathrm{PhD}$ thesis and I am grateful for that. Furthermore I'd like to thank Sarah Köster, Theo Geisel, Ulrich Parlitz, Wolfram Kollatschny and Jens Niemeyer to be in my thesis examination committee.

I cannot emphasize enough the help of Albert J. Bae. He taught me MATLAB, a lot of physics, as well as english and he contributed a large amount of work and ideas to both the flattening and the periodic forcing project. Furthermore we shared the same sense of tradition, as we visited a Subway restaurant each Wednesday and an asian restaurant each Friday. Yet another tradition I shared with Stephan Weiss, whom I met every Monday for the Conchita pizza seminar to discuss astrophysics (we shared the same minor subject), correct student homework or talk about life in general. As Stephan pointed out in his thesis acknowledgement already: It was one of the highlights of each week. I also need to point out my second GAUSS-PhD fellow Gabriel Amselem, who helped a lot with MATLAB and with whom I visited numerous student parties in Göttingen. I definitely would like to thank him once more, that he allowed me to celebrate my 30th birthday party in his (!) apartment. I am very thankful to Marco Tarantola. He joined the group at a critical stage, right after Albert and Gabriel left and right before the move to the new institute building. Together with him and Katharina Schneider we kept the Dicty group running. Furthermore I'd like to thank him for contributing his knowledge about atomic force microscopy to the projects, for leading the Dicty group and for proofreading the manuscript. Especially appreciated is his 
help in finalizing the manuscript of this thesis. Many thanks to my former Bachelor-student Rabea Sandmann. After just a few weeks she worked straightforward on her own and contributed greatly to the periodic forcing experiments. José Negrete Jr. joined the Dicty group in early 2011, but already within his first year he contributed much of his knowledge about dynamical systems to the periodic forcing project. Furthermore I am grateful for his help in the preparation of the nonlinear dynamics examination. Matthias Theves, a former diploma student contributed the cell tracking algorithm to the periodic forcing data analysis. $\mathrm{He}$ was a good companion over many weekends, especially in advance of conference talks. I'd like to thank the other group members of the Dicty group, namely Vladimir Zykov, Azam Gholami, Christoph Blum (also for proofreading), Hsin-Fang Hsu, Noriko Oikawa, Weixing Li for a nice working atmosphere over several years and in different offices. I mentioned Katharina Schneider already, but would like to furthermore state that I am very grateful for her work in the biology laboratory. She kept the laboratory running and with her and her enthusiasm we extended the cell culture lab to a functional molecular biology lab. Aside from work I always enjoyed chatting with her. Further thanks are addressed to Barbara Kasemann, Jan Sebastian Wittwer, Stefanie Gebel, Valerie Biewener and Kerstin Eckelmann for valuable technical assistance. I'd like to thank Andreas Renner, Artur Kubitzek and Andreas Kopp for help and support considering the MPI-DS cleanroom facility. Special thanks to Angela Meister our secretary, who took care of numerous organisatorial issues. Gerhard Nolte our system administrator was very helpful considering all hardware and software based issues. Olympus contributed valuable support for the light and laser microscopes by Helge Schmidt, Hendrik Deubel and Thomas Wenninger. Helge Schmidt, I'd like to thank in particular because we taught the course on confocal laser scanning microscopy together in 2010.

I am grateful to get to know and to discuss with the other former and present scientists of the Bodenschatz department: Kelken Chang, Michael Sinhuber, Mathieu Gibert (also for his suggestions on frequency analysis), Mireia Torralba, Haitao Xu, Dario Vincenzi, Holger Nobach, Florian Winkel, Simon Klein, Simon Schütz, Steffen Risius, Fabio di Lorenzo, Xiaozhou He, Ewe-Wei Saw, Sebastian Lambertz, Hengdong Xi, Walter Pauls, Huixuan Wu, Denis Funfschilling and Gabriel Seiden. Especially I always appreciated to share thougths and news with my $>5$ year fellows Gregory Bewley from the turbulence and Claudia Richter from the biomedical physics group. Further former and present members of the biomedical physics group, which I'd like to mention, are Amgad Squires (always a source of good ideas, considering MATLAB programming), Philip Bittihn, Alexander Ahlborn, Johannes Schröder-Schetelig (very useful advices considering high-speed camera imaging) and the head of the group Stefan Luther. 
I'd like to thank the former diploma student Robert Zimmermann. His advices on the periodic forcing project were very valuable and I am furthermore grateful for his help preparing the student lab courses. I have to thank Jennifer Mutschall for her enthusiastic help with $\mathrm{ITT}_{\mathrm{E}} \mathrm{X}$ and for considerably improving the style of this $\mathrm{PhD}$ thesis. I'm also very grateful for the help of Katherine R. Thomas. Her comments regarding the english and the science were very useful. Holly Capelo also helped improving the english and furthermore I am thankful for her help, preparing the examination in astrophysics. The person to ask in terms of chemistry was Julie L. Murison and she also organized fantastic (and new to me) party events, as well as introduced australian music acts to me. Edouard Galland, a former intern from the École polytechnique, explained microfluidic techniques to me and developed the first closed-end microfluidic actuator.

The characterization of the caged cAMP compounds was a crucial part of this thesis and I would like to thank Ullrich Benjamin Kaupp, Nico Kotzur, Timo Strünker, Luis Alvarez (all CAESAR, Bonn) as well as Claudia Höbartner (MPI BPC, Göttingen) for making their HPLC available and for further suggestions on the treatment of caged compounds. Hellen C. Ishikawa-Ankerhold (now LMU Munich) started the research on self sustained actin oscillations in the D. discoideum SCAR(-)/PIR121(-) knockout mutant. I continued with this project and I would like to thank here for advices and critical reading of the manuscript. Her PhD advisor Günter Gerisch (MPI Biochemistry, Martinsried) was the main source of mutant strains, used in this work. Furthermore I would like to thank him for valuable discussions on the periodic forcing project. For suggestions on the periodic forcing chapter, I'd like to thank Kees Weijer (University of Dundee, Scotland, UK) and Fred Wolf (MPI DS, Göttingen)

Over the course of this thesis two interesting scientific collaborations emerged. The first one on the collective oscillations in D. discoideum was mentioned in this thesis (see section 4.3) and I would like to thank Edith Schäfer, Burkhard Geil and Andreas Janshoff for the great teamwork. Furthermore, I would like to mention Regina Faubel and her supervisor Gregor Eichele (both MPI BPC Göttingen). Within the last year we set up a very interesting project, considering the cilia beating of the brain ventricular system and I am very much looking forward to continue research on this.

I was given the possibility to join the PHYSBIO 2007 summer school, which took place in St. Etienne de Tinée (France). This was a great experience and I thank Albrecht Ott and Jean-Luc Beaumont for organizing this excellent summer school. Furthermore I'd like to thank Jitka C̆jeková, András Czovek, Judith Varnai, Gergely J. Szollosi and Franziska Lautenschläger for organizing the PHYSBIO revival retreats in Prague, Budapest and Leipzig. Special thanks to Christoph Erlenkämper, whom I got to know during the PHYSBIO sum- 
mer school. He has become a good friend and furthermore helped a lot with the construction and the final characterization of the flattening devices. Special thanks to Larisa Yurlova for continuously inviting me to the previously mentioned numerous student parties. Other colleges, with whom I shared the latest developments in my thesis and whom I'd like to thank for their patience listening to me are: Sarah G. Schwarz Henriques, Kathy Gelato, Jannick Langfahl-Klabes, Quentin Brosseau, Martha Brennich, Jean-François Métayer, Nachiket Kashikar, Annika Döring and Max Neudecker.

For great support and motivation over many years I like to thank my former study colleagues and friends Christin Zachow, Henry Müller, Julia and Christoph Scherber, Florian Nagel, Anja May, Oliver Riebe, Falk Hillmann, Katja Klausz, Katja Muders, Diana Buss, as well as my former supervisor Gabriele Berg. Special thanks to Christin and Henry, who live and work in Graz and whom I visited at least once every year of my $\mathrm{PhD}$ thesis. I can not emphasize enough the help of Sindy Gebhard and Susann Schindler over the years we know each other. Also I would like to mention my long-time friends from school Björn Labuzinski and Thomas Brecheis as well as Marc Buck. For his continuous support over more than 4 years, I would like to acknowledge Dr. med. Klaus Radau-Pfeil. My final thanks are addressed to my family, my parents Christina and Hans-Joachim and my brother Norbert for their love, their support and help.

This work was funded by the Max Planck Society as well as the German Science Foundation in the framework of the SPP 1128 and the SFB 937. 
Appendix

\section{A. Supplementary material on CD-ROM}

- cfluorescein-uncaging.avi - Fluorescein release from DMNB-caged precursor.

DMNB-caged fluorescein is flown in a microfluidic channel and fluorescein is released by laser-mediated photolysis right in front of the imaging region. The movie corresponds to the obtained fluorescent profile in figure 2.5A. Images recorded at $10 \mathrm{~Hz}$ and displayed with $10 \mathrm{fps}$. Flow from left to right. Scale bar is $10 \mu \mathrm{m}$.

- TIRF-LimEGFP.avi - TIRF microcopy of LimE-GFP under flattening.

A single layer microfluidic compression chamber was used to flatten a $D$. discoideum LimE-GFP cell, confining it to the glass boundary. GFP is excited by the evanescent wave of a $488 \mathrm{~nm}$ Ar-laser. Images sampled with $2 \mathrm{~Hz}$ and displayed at $20 \mathrm{fps}$. Scale bar is $10 \mu \mathrm{m}$.

- Fluorescein-compression.avi - Visualization of the microfluidic compression.

The lower channel of this through-flow double layer device is filled with $100 \mu \mathrm{M}$ fluorescein and a volume flow of $5 \mu \mathrm{l} / \mathrm{h}$ is engaged. The fluorescein solution is excited by a MT20 burner. Changing the pressure in the actuation channel changes the height of the lower channel, decreasing the fluorescence intensity. Images were recorded with $1 \mathrm{~Hz}$ and are displayed with $10 \mathrm{fps}$. 


\section{B. MATLAB script for DDE simulation}

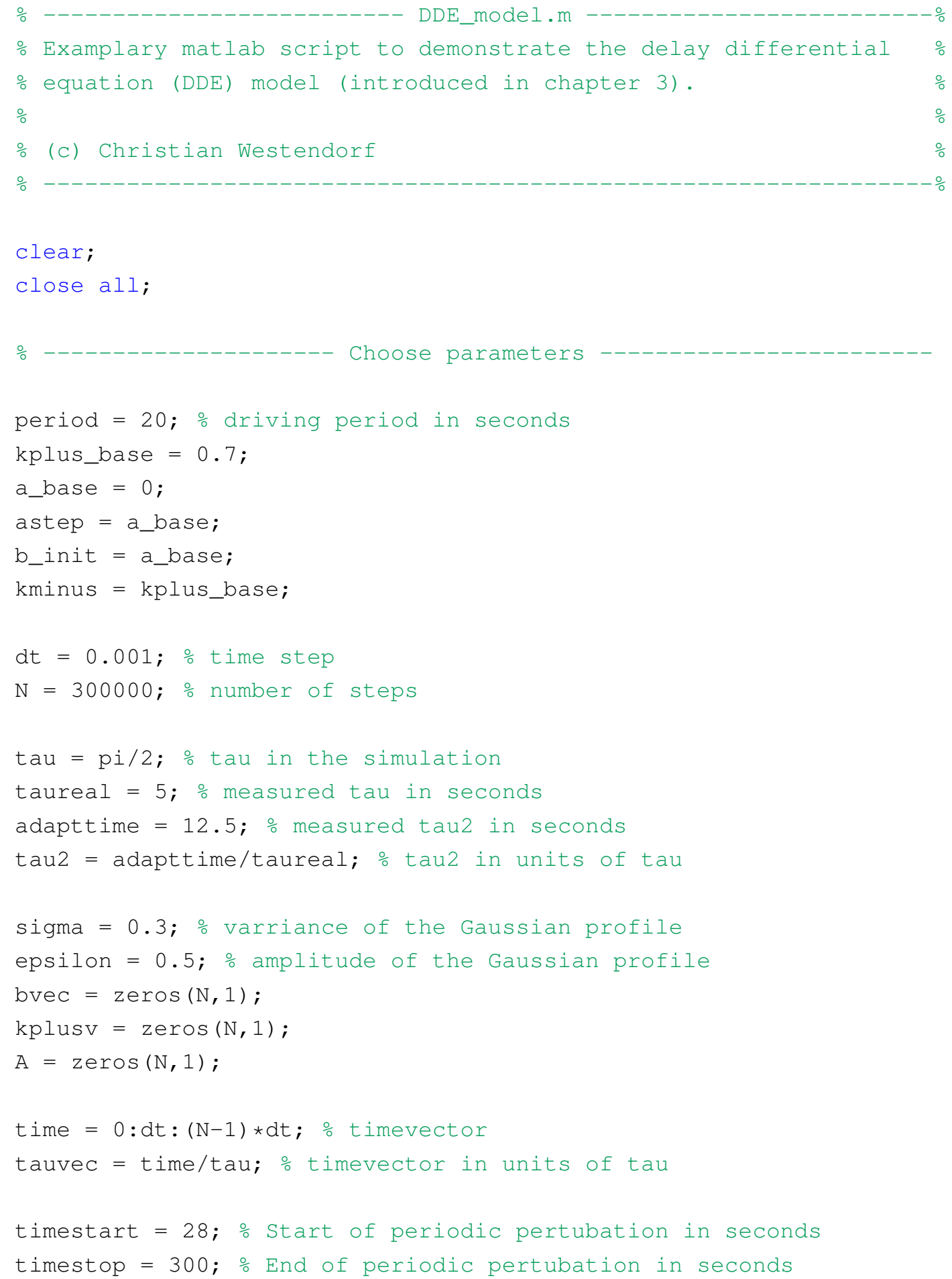




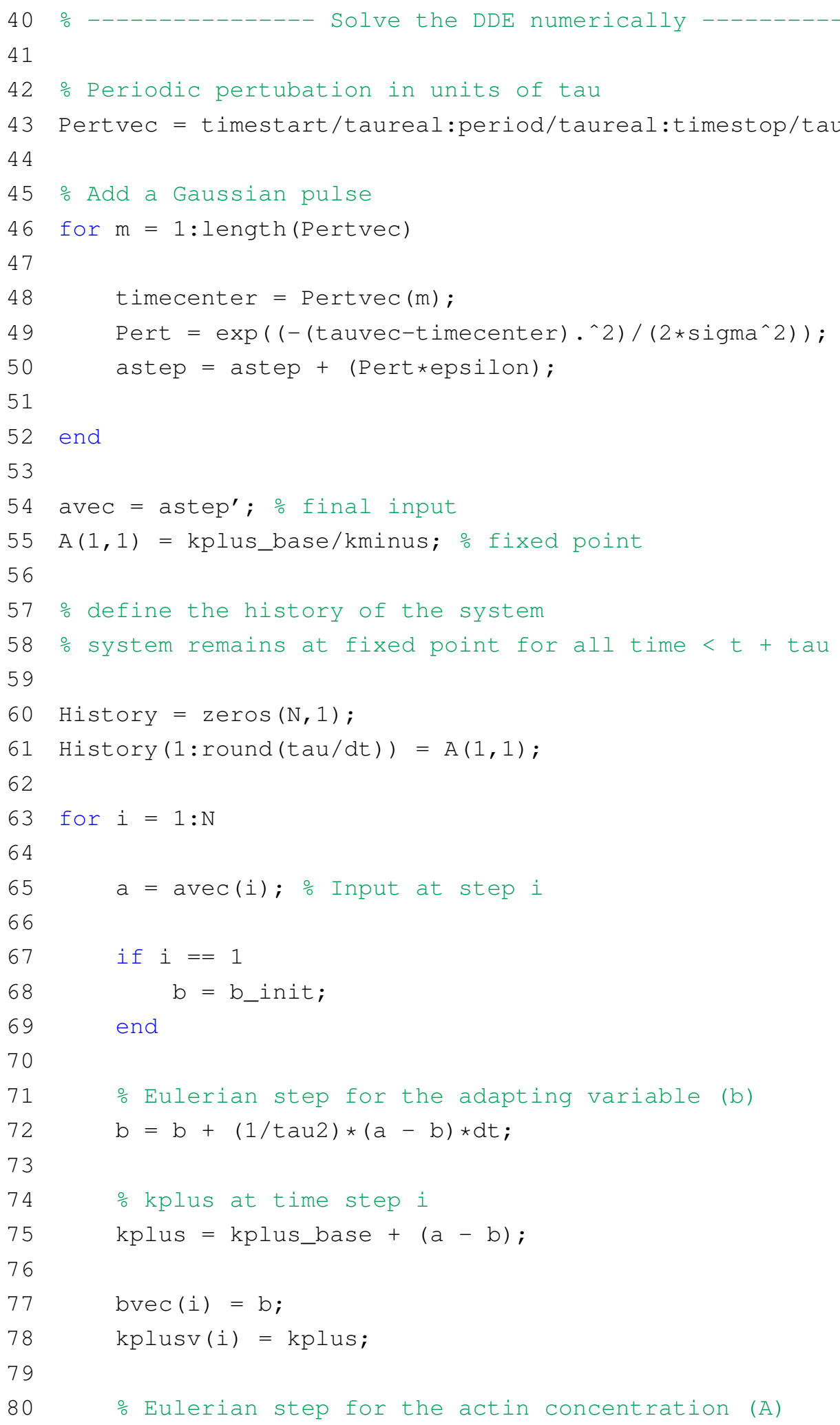




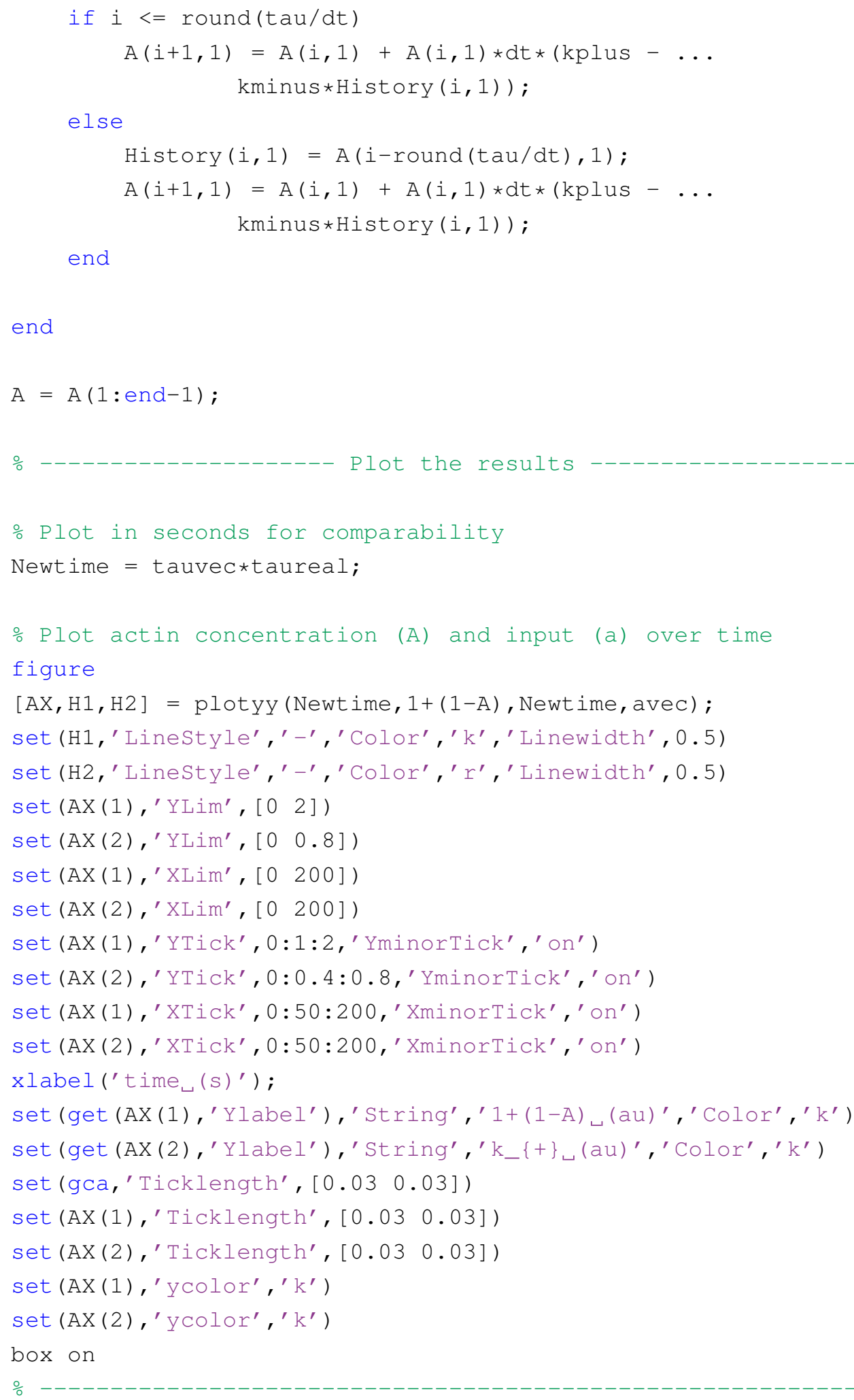




\section{Bibliography}

Amselem, G. (2010). Directional sensing and chemotaxis in eukaryotic cells - a quantitative study. PhD thesis, Georg August University Goettingen.

Andrew, N. and Insall, R. H. (2007). Chemotaxis in shallow gradients is mediated independently of PtdIns 3-kinase by biased choices between random protrusions. Nature Cell Biology, 9(2):193-U91.

Ashworth, J. and Watts, D. (1970). Metabolism of cellular slime mould Dictyosteliumdiscoideum grown in axenic culture. Biochemical Journal, 119(2):175-\&.

Aufderheide, K. (1986). Identification of the basal bodies and kinetodesmal fibers in living cells of Paramecium-tetraurelia sonneborn, 1975 and Paramecium-sonneborni Aufderheide, Daggett + Nerad, 1983. Journal of Protozoology, 33(1):77-80.

Aufderheide, K. J. (2008). An overview of techniques for immobilizing and viewing living cells. Micron, 39(2):71-76.

Axelrod, D. (1981). cell-substrate contacts illuminated by total internal-reflection fluorescence. Journal of Cell Biology, 89(1):141-145.

Bae, A. J. (2010). Explorations into the life of Dictyostelium discoideum. $\mathrm{PhD}$ thesis, Cornell University, Ithaca, NY.

Bae, A. J., Beta, C., and Bodenschatz, E. (2009). Rapid switching of chemical signals in microfluidic devices. Lab on a Chip, 9(21):3059-3065. 
Bae, A. J. and Bodenschatz, E. (2010). On the swimming of Dictyostelium amoebae. Proceedings of the National Academy of Sciences of the United States of America, 107(44):E165-E166.

Baggiolini, M. (1998). Chemokines and leukocyte traffic. Nature, 392(6676):565-568.

Bagorda, A. and Parent, C. A. (2008). Eukaryotic chemotaxis at a glance. Journal of Cell Science, 121(16):2621-2624.

Barltrop, J., Plant, P., and P, S. (1966). Photosensitive protective groups. Chemical Communications, 22:822-\&.

Barry, N. P. and Bretscher, M. S. (2010). Dictyostelium amoebae and neutrophils can swim. Proceedings of the National Academy of Sciences of the United States of America, 107(25):11376-11380.

Bear, J., Rawls, J., and Saxe, C. (1998). SCAR, a WASP-related protein, isolated as a suppressor of receptor defects in late Dictyostelium development. Journal of Cell Biology, 142(5):1325-1335.

Berg, H. (2003). The rotary motor of bacterial flagella. Annual Review of Biochemistry, 72:19-54.

Beta, C. (2009). Chemotaxis: Methods and protocols, volume 571 of Methods in Molecular Biology, pages 321-332. Humana Press Inc, 999 Riverview Dr, Ste 208, totowa, NJ 07512-1165 USA.

Beta, C., Froehlich, T., Boedeker, H. U., and Bodenschatz, E. (2008). Chemotaxis in microfluidic devices - a study of flow effects. Lab on a Chip, 8(7):1087-1096.

Beta, C., Wyatt, D., Rappel, W.-J., and Bodenschatz, E. (2007). Flow photolysis for spatiotemporal stimulation of single cells. Analytical Chemistry, 79(10):3940-3944.

Blagg, S. and Insall, R. (2004). Solving the WAVE function. Nature Cell Biology, 6(4):279281.

Blagg, S., Stewart, M., Sambles, C., and Insall, R. (2003). PIR121 regulates pseudopod dynamics and SCAR activity in Dictyostelium. Current Biology, 13(17):1480-1487.

Boenigk, W., Loogen, A., Seifert, R., Kashikar, N., Klemm, C., Krause, E., Hagen, V., Kremmer, E., Struenker, T., and Kaupp, U. B. (2009). An Atypical CNG Channel Activated by a Single cGMP Molecule Controls Sperm Chemotaxis. Science Signaling, 2(94). 
Bonner, J. (1998). A way of following individual cells in the migrating sings of Dictyostelium discoideum. Proceedings of the National Acadamy of Sciences of the United States of America, 95(16):9355-9359.

Bonner, J., Segel, L., and Cox, E. (1998). Oxygen and differentiation in Dictyostelium discoideum. Journal of Biosciences, 23(3):177-184.

Bosgraaf, L. and Van Haastert, P. J. M. (2009). Navigation of chemotactic cells by parallel signaling to pseudopod persistence and orientation. PLOS one, 4(8).

Bretschneider, T., Anderson, K., Ecke, M., Mueller-Taubenberger, A., Schroth-Diez, B., Ishikawa-Ankerhold, H. C., and Gerisch, G. (2009). The three-dimensional dynamics of actin waves, a model of cytoskeletal self-organization. Biophysical Journal, 96(7):28882900.

Bretschneider, T., Diez, S., Anderson, K., Heuser, J., Clarke, M., Muller-Taubenberger, A., Kohler, J., and Gerisch, G. (2004). Dynamic actin patterns and Arp2/3 assembly at the substrate-attached surface of motile cells. Current Biology, 14(1):1-10.

Brooks, F. J. and Carlsson, A. E. (2010). Actin polymerization overshoots induced by plusend capping. Physical Biology, 7(1).

Bukahrova, T., Weijer, G., Bosgraaf, L., Dormann, D., van Haastert, P., and Weijer, C. (2005). Paxillin is required for cell-substrate adhesion, cell sorting and slug migration during Dictyostelium development. Journal of Cell Science, 118(18):4295-4310.

Buzan, J. and Frieden, C. (1996). Yeast actin: Polymerization kinetic studies of wild type and a poorly polymerizing mutant. Proceedings of the National Academy of Sciences of the United States of America, 93(1):91-95.

Camalet, S., Duke, T., Julicher, F., and Prost, J. (2000). Auditory sensitivity provided by self-tuned critical oscillations of hair cells. Proceedings of the National Academy of Sciences of the United States of America, 97(7):3183-3188.

Carlsson, A. E. (2010). Actin dynamics: From nanoscale to microscale. Annual Review of Biophysics, 39:91-110.

Carlsson, L., Nystrom, L., Sundkvist, I., Markey, F., and Lindberg, U. (1977). Actin polymerizability is influenced by profilin, a low-molecular weight protein in non-muscle cells. Journal of Molecular Biology, 115(3):465-483. 
Caterina, M., Hereld, D., and Devreotes, P. (1995). Occupancy of the dictyostelium cAMP receptor, cAR1, induces a reduction in affinity which depends upon cooh-terminal serine residues. Journal of Biological Chemistry, 270(9):4418-4423.

Celani, A. and Vergassola, M. (2010). Bacterial strategies for chemotaxis response. Proceedings of the National Academy of Sciences of the United States of America, 107(4):1391-1396.

Chan, T. and Vese, L. (2001). Active contours without edges. IEEE Transactions on Image Processing, 10(2):266-277.

Chen, L., Iijima, M., Tang, M., Landree, M. A., Huang, Y. E., Xiong, Y., Iglesias, P. A., and Devreotes, P. N. (2007). PLA(2) and PI3K/PTEN pathways act in parallel to mediate chemotaxis. Developmental Cell, 12(4):603-614.

Chen, L., Janetopoulos, C., Huang, Y., Iijima, M., Borleis, J., and Devreotes, P. (2003). Two phases of actin polymerization display different dependencies on PI(3,4,5)P-3 accumulation and have unique roles during chemotaxis. Molecular Biology of the Cell, 14(12):5028-5037.

Chen, Z., Borek, D., Padrick, S. B., Gomez, T. S., Metlagel, Z., Ismail, A. M., Umetani, J., Billadeau, D. D., Otwinowski, Z., and Rosen, M. K. (2010). Structure and control of the actin regulatory WAVE complex. Nature, 468(7323):533-U207.

Chien, S., Chung, C., Sukumaran, S., Osborne, N., Lee, S., Ellsworth, C., McNally, J., and Firtel, R. (2000). The Dictyostelium LIM domain-containing protein LIM2 is essential for proper chemotaxis and morphogenesis. Molecular Biology of the Cell, 11(4):12751291.

Chisholm, R. and Firtel, R. (2004). Insights into morphogenesis from a simple developmental system. Nature Reviews Molecular Cell Biology, 5(7):531-541.

Chisholm, R., Hopkinson, S., and Lodish, H. (1987). Superinduction of the Dictyostelium discoedium cell surface cAMP receptor by pulses of cAMP. Proceedings of the National Acadamy of Sciences of the United States of America, 84(4):1030-1034.

Clarke, M. and Maddera, L. (2006). Phagocyte meets prey: Uptake, internalization, and killing of bacteria by Dictyostelium amoebae. European Journal of Cell Biology, 85(910):1001-1010. 
Condeelis, J., Singer, R., and Segall, J. (2005). The great escape: When cancer cells hijack the genes for chemotaxis and motility. Annual Review of Cell and Developmental Biology, 21:695-718.

Cooper, J. (1987). Effects of Cytochalasin and Phalloidin on Actin. Journal of Cell Biology, 105(4):1473-1478.

Crone, S. and Lee, K. (2002). The bound leading the bound: Target-derived receptors act as guidance cues. Neuron, 36(3):333-335.

Dalous, J., Burghardt, E., Mueller-Taubenberger, A., Bruckert, F., Gerisch, G., and Bretschneider, T. (2008). Reversal of cell polarity and actin-myosin cytoskeleton reorganization under mechanical and chemical stimulation. Biophysical Journal, 94(3):10631074.

Davidson, A. J. and Insall, R. H. (2011). Actin-based motility: WAVE regulatory complex structure reopens old SCARs. Current Biology, 21(2):R66-R68.

de la Roche, M. and Cote, G. (2001). Regulation of Dictyostelium Myosin I and II. Biochimica et Biophysica Acta-general Subjects, 1525(3):245-261.

DeHostos, E., Bradtke, B., Lottspeich, F., Guggenheim, R., and Gerisch, G. (1991). Coronin, an actin binding-protein of Dictyostelium-discoideum localized to cell-surface projections, has sequence similarities to G-protein beta-subunits. EMBO Journal, 10(13):4097-4104.

Depraitere, C. and Darmon, M. (1978). Growth of Dictyostelium-discoideum on different species of bacteria. Annales de Microbiologie, B129(3):451-\&.

Devreotes, P. and Janetopoulos, C. (2003). Eukaryotic chemotaxis: Distinctions between directional sensing and polarization. Journal of Biological Chemistry, 278(23):2044520448.

Dewitt, S., Darley, R. L., and Hallett, M. B. (2009). Translocation or just location? Pseudopodia affect fluorescent signals. Journal of Cell Biology, 184(2):197-203.

Diez, S., Gerisch, G., Anderson, K., Muller-Taubenberger, A., and Bretschneider, T. (2005). Subsecond reorganization of the actin network in cell motility and chemotaxis. Proceedings of the National Academy of Sciences of the United States of America, 102(21):76017606. 
Dormann, D., Kim, J., Devreotes, P., and Weijer, C. (2001). cAMP receptor affinity controls wave dynamics, geometry and morphogenesis in Dictyostelium. Journal of Cell Science, 114(13):2513-2523.

Dworkin, M. and Keller, K. (1977). Solubility and diffusion-coefficient of adenosine 3'-5'monophosphate. Journal of Biological Chemistry, 252(3):864-865.

Eden, S., Rohatgi, R., Podtelejnikov, A., Mann, M., and Kirschner, M. (2002). Mechanism of regulation of WAVE1-induced actin nucleation by Rac1 and Nck. Nature, 418(6899):790-793.

Eichinger, L., Pachebat, J., Glockner, G., Rajandream, M., Sucgang, R., Berriman, M., Song, J., Olsen, R., Szafranski, K., Xu, Q., Tunggal, B., Kummerfeld, S., Madera, M., Konfortov, B., Rivero, F., Bankier, A., Lehmann, R., Hamlin, N., Davies, R., Gaudet, P., Fey, P., Pilcher, K., Chen, G., Saunders, D., Sodergren, E., Davis, P., Kerhornou, A., Nie, X., Hall, N., Anjard, C., Hemphill, L., Bason, N., Farbrother, P., Desany, B., Just, E., Morio, T., Rost, R., Churcher, C., Cooper, J., Haydock, S., van Driessche, N., Cronin, A., Goodhead, I., Muzny, D., Mourier, T., Pain, A., Lu, M., Harper, D., Lindsay, R., Hauser, H., James, K., Quiles, M., Babu, M., Saito, T., Buchrieser, C., Wardroper, A., Felder, M., Thangavelu, M., Johnson, D., Knights, A., Loulseged, H., Mungall, K., Oliver, K., Price, C., Quail, M., Urushihara, H., Hernandez, J., Rabbinowitsch, E., Steffen, D., Sanders, M., Ma, J., Kohara, Y., Sharp, S., Simmonds, M., Spiegler, S., Tivey, A., Sugano, S., White, B., Walker, D., Woodward, J., Winckler, T., Tanaka, Y., Shaulsky, G., Schleicher, M., Weinstock, G., Rosenthal, A., Cox, E., Chisholm, R., Gibbs, R., Loomis, W., Platzer, M., Kay, R., Williams, J., Dear, P., Noegel, A., Barrell, B., and Kuspa, A. (2005). The genome of the social amoeba Dictyostelium discoideum. Nature, 435(7038):43-57.

Enculescu, M. and Falcke, M. (2011). Actin-based propulsion of spatially extended objects. New Journal of Physics, 13.

Engels, J. and Schlaeger, E. (1977). Synthesis, structure, and reactivity of Adenosine cyclic 3',5'-phosphate benzyl triesters. Journal of Medicinal Chemistry, 20(7):907-911.

Etzrodt, M., Ishikawa, H. C. F., Dalous, J., Mueller-Taubenberger, A., Bretschneider, T., and Gerisch, G. (2006). Time-resolved responses to chemoattractant, characteristic of the front and tall of Dictyostelium cells. FEBS Letters, 580(28-29):6707-6713.

Evangelista, M., Zigmond, S., and Boone, C. (2003). Formins: signaling effectors for assembly and polarization of actin filaments. Journal of Cell Science, 116(13):26032611. 
Feneberg, W., Westphal, M., and Sackmann, E. (2001). Dictyostelium cells' cytoplasm as an active viscoplastic body. European Biophysics Journal with Biophysics Letters, 30(4):284-294.

Fey, P., Kowal, A. S., Gaudet, P., Pilcher, K. E., and Chisholm, R. L. (2007). Protocols for growth and development of Dictyostelium discoideum. Nature Protocols, 2(6):13071316.

Filic, V., Marinovic, M., Faix, J., and Weber, I. (2012). A dual role for Rac1 GTPases in the regulation of cell motility. Journal of Cell Science, 125(2):387-398.

Fisher, P., Smith, E., and Williams, K. (1981). An extracellular chemical signal controlling phototactic behavior by D-discoideum slugs. Cell, 23(3):799-807.

Fleming, S. and Rieder, C. (2003). Flattening Drosophila cells for high-resolution light microscopic studies of mitosis in vitro. Cell Motility and the Cytoskeleton, 56(3):141146.

Flyvbjerg, H. and Petersen, H. (1989). Error-estimates on averages of correlated data. Journal of Chemical Physics, 91(1):461-466.

Franca-Koh, J. and Devreotes, P. (2004). Moving forward: Mechanisms of chemoattractant gradient sensing. Physiology, 19:300-308.

Franke, J. and Kessin, R. (1977). Defined minimal medium for axenic strains of Dictyostelium-discoideum. Proceedings of the National Academy of Sciences of the United States of America, 74(5):2157-2161.

Freyd, G., Kim, S., and Horvitz, H. (1990). Novel cysteine-rich motif and homeodomain in the product of the Caenorhabditis-elegans cell lineage gene LIN-II. Nature, 344(6269):876-879.

Fukui, Y., Yumura, S., Yumura, T., and Mori, H. (1986). Agar overlay method - highresolution immunofluorescence for the study of the contractile apparatus. Methods in Enzymology, 134:573-580.

Fuller, D., Chen, W., Adler, M., Groisman, A., Levine, H., Rappel, W.-J., and Loomis, W. F. (2010). External and internal constraints on eukaryotic chemotaxis. Proceedings of the National Academy of Sciences of the United States of America, 107(21):9656-9659. 
Futrelle, R., Traut, J., and Mckee, W. (1982). Cell behavior in Dictyostelium-discoideum - pre-aggregation response to localized cyclic-AMP pulses. Journal of Cell Biology, 92(3):807-821.

Gerisch, G. (2008). Personal communication.

Gerisch, G., Albrecht, R., Heizer, C., Hodgkinson, S., and Maniak, M. (1995). Chemoattractant-controlled accumulation of coronin at the leading-edge of Dictyostelium cells monitored using green fluorescent protein-coronin fusion protein. Current Biology, 5(11):1280-1285.

Gerisch, G. and Wick, U. (1975). Intracellular oscillations and release of cyclic-AMP from Dictyostelium cells. Biochemical and Biophysical Research Communications, 65(1):364-370.

Getreuer, P. (2010). tvreg: Variational image restoration and segmentation.

Gholami, A., Falcke, M., and Frey, E. (2008). Velocity oscillations in actin-based motility. New Journal of Physics, 10.

Giaever, I. and Keese, C. (1993). A morphological biosensor for mammalian-cells. Nature, 366(6455):591-592.

Giannone, G., Dubin-Thaler, B., Dobereiner, H., Kieffer, N., Bresnick, A., and Sheetz, M. (2004). Periodic lamellipodial contractions correlate with rearward actin waves. CELL, 116(3):431-443.

Gillies, N., Hariratnajothi, N., and Ong, C. (1976). Comparison of sensitivity of spores and amebas of Dictyostelium-discoideum to gamma-rays and ultraviolet-light. Journal of General Microbiology, 92(JAN):229-233.

Goley, E. D. and Welch, M. D. (2006). The ARP2/3 complex: an actin nucleator comes of age. Nature Reviews Molecular Cell Biology, 7(10):713-726.

Gomer, R. and Firtel, R. (1987). Cell-autonomous determination of cell-type choice in Dictyostelium development by cell-cycle phase. Science, 237(4816):758-762.

Gonzales, R., Woods, R., and Eddins, S. (2004). Digital image processing using MATLAB. Pearson Prentice Hall, Upper Saddle River, New Jersey, NY, USA.

Goode, B. L. and Eck, M. J. (2007). Mechanism and function of formins in the control of actin assembly. Annual Review of Biochemistry, 76:593-627. 
Hacker, U., Albrecht, R., and Maniak, M. (1997). Fluid-phase uptake by macropinocytosis in Dictyostelium. Journal of Cell Science, 110(Part 2):105-112.

Hagen, V Benndorf, K. and Kaupp, U. (2005). Control of cellular activity, chapter 4, pages 155-178. Blackwell Science Publ, Osney Mead, Oxford OX2 0EL, UK.

Hagen, V., Bendig, J., Frings, S., Eckardt, T., Helm, S., Reuter, D., and Kaupp, U. (2001). Highly efficient and ultrafast phototriggers for cAMP and cGMP by using longwavelength UV/vis-activation. Angewandte Chemie-International Edition, 40(6):1046+.

Hagen, V., Dzeja, C., Frings, S., Bendig, J., Krause, E., and Kaupp, U. (1996). Caged compounds of hydrolysis-resistant analogues of cAMP and cGMP: Synthesis and application to cyclic nucleotide-gated channels. Biochemistry, 35(24):7762-7771.

Hagen, V., Frings, S., Bendig, J., Lorenz, D., Wiesner, B., and Kaupp, U. (2002). Fluorescence spectroscopic quantification of the release of cyclic nucleotides from photocleavable [bis(carboxymethoxy)coumarin-4-yl]methyl esters inside cells. Angewandte Chemie-International Edition, 41(19):3625-3628.

Hall, A., Schlein, A., and Condeelis, J. (1988). Relationship of pseudopod extension to chemotactic hormone-induced actin polymerization in ameboid cells. Journal of Cellular Biochemistry, 37(3):285-299.

Heinrich, D., Youssef, S., Schroth-Diez, B., Engel, U., Aydin, D., Bluemmel, J., Spatz, J. P., and Gerisch, G. (2008). Actin-cytoskeleton dynamics in non-monotonic cell spreading. Cell Adhesion \& Migration, 2(2):58-68.

Hu, Z. and Lutkenhaus, J. (1999). Topological regulation of cell division in Escherichia coli involves rapid pole to pole oscillation of the division inhibitor MinC under the control of MinD and MinE. Molecular Microbiology, 34(1):82-90.

Huang, W., Ramsey, K. M., Marcheva, B., and Bass, J. (2011). Circadian rhythms, sleep, and metabolism. Journal of Clinical Investigation, 121(6):2133-2141.

Ibarra, N., Pollitt, A., and Insall, R. (2005). Regulation of actin assembly by SCAR/WAVE proteins. Biochemical Society Transactions, 33(Part 6):1243-1246.

Innocenti, M., Zucconi, A., Disanza, A., Frittoli, E., Areces, L., Steffen, A., Stradal, T., Di Fiore, P., Carlier, M., and Scita, G. (2004). Abi1 is essential for the formation and activation of a WAVE2 signalling complex. Nature Cell Biology, 6(4):319-327. 
Insall, R. H. and Machesky, L. M. (2009). Actin dynamics at the leading edge: From simple machinery to complex networks. Developmental Cell, 17(3):310-322.

Ishikawa-Ankerhold, C. H. (2008). Functional organization of the actin system in Dictyostelium. PhD thesis, Technical university Munich, Munich, Germany.

Ismail, A. M., Padrick, S. B., Chen, B., Umetani, J., and Rosen, M. K. (2009). The WAVE regulatory complex is inhibited. Nature Structural \& Molecular Biology, 16(5):561-563.

Iwadate, Y. and Yumura, S. (2008). Actin-based propulsive forces and myosin-II-based contractile forces in migrating Dictyostelium cells. Journal of Cell Science, 121(8):13141324.

Janetopoulos, C. and Firtel, R. A. (2008). Directional sensing during chemotaxis. FEBS Letters, 582(14):2075-2085.

Janssens, P. and VanHaastert, P. (1987). Molecular-basis of transmembrane signal transduction in dictyostelium-discoideum. Microbiological Reviews, 51(4):396-418.

Jin, T. (2009). Chemotaxis: Methods and protocols. Series: Methods in Molecular Biology.

Johnson, R., VanHaastert, P., Kimmel, A., Saxe, C., Jastorff, B., and Devreotes, P. (1992). The cyclic-nucleotide specificity of 3 cAMP receptors in Dictyostelium. Journal of Biological Chemistry, 267(7):4600-4607.

Julicher, F. and Prost, J. (1997). Spontaneous oscillations of collective molecular motors. Physical Review Letters, 78(23):4510-4513.

Kaplan, J., Forbush, B., and Hoffman, J. (1978). Rapid photolytic release of Adenosine 5'triphosphate from a protected analog - utilization by Na-K pump of human red blood-cell ghosts. Biochemistry, 17(10):1929-1935.

Karpen, J., Zimmerman, A., Stryer, L., and Baylor, D. (1988). Gating kinetics of the cyclic-GMP-activated channel of retinal rods - flash-photolysis and voltage-jump studies. Proceedings of the National Academy of Sciences of the United States of America, 85(4):1287-1291.

Karra, S. and Karim, M. N. (2009). Comprehensive methodology for detection and diagnosis of oscillatory control loops. Control Engineering Practice, 17(8):939-956.

Kessin, R. H. (2001). Dictyostelium: evolution, cell biology, and the development of multicellularity. Cambridge University Press, Cambridge, UK. 
Khurana, B., Khurana, T., Khaire, N., and Noegel, A. (2002a). Functions of LIM proteins in cell polarity and chemotactic motility. EMBO Journal, 21(20):5331-5342.

Khurana, T., Khurana, B., and Noegel, A. (2002b). LIM proteins: association with the actin cytoskeleton. Protoplasma, 219(1-2):1-12.

Kim, Y. C., Kang, J. H., Park, S.-J., Yoon, E.-S., and Park, J.-K. (2007). Microfluidic biomechanical device for compressive cell stimulation and lysis. Sensors and Actuators B-Chemical, 128(1):108-116.

Kitanishiyumura, T. and Fukui, Y. (1989). Actomyosin organization during cytokinesis reversible translocation and differential redistribution in Dictyostelium. Cell Motility and the Cytoskeleton, 12(2):78-89.

Konzok, A., Weber, I., Simmeth, E., Hacker, U., Maniak, M., and Muller-Taubenberger, A. (1999). DAip1, a Dictyostelium homologue of the yeast actin-interacting protein 1, is involved in endocytosis, cytokinesis, and motility. Journal of Cell Biology, 146(2):453464.

Kreppel, L., Fey, P., Gaudet, P., Just, E., Kibbe, W., Chisholm, R., and Kimmel, A. (2004). dictyBase: a new Dictyostelium discoideum genome database. Nucleic Acids Research, 32(SI):D332-D333.

Kruse, K. and Juelicher, F. (2005). Oscillations in cell biology. Current Opinion in Cell Biology, 17(1):20 - 26.

Kubo, R. (1957). Statistical-mechanical theory of irreversible processes .1. general theory and simple applications to magnetic and conduction problems. Journal of the Physical Society of Japan, 12(6):570-586.

Kunda, P., Craig, G., Dominguez, V., and Baum, B. (2003). Abi, Sra1, and Kette control the stability and localization of SCAR/WAVE to regulate the formation of actin-based protrusions. Current Biology, 13(21):1867-1875.

Lacayo, C. I., Soneral, P. A. G., Zhu, J., Tsuchida, M. A., Footer, M. J., Soo, F. S., Lu, Y., Xia, Y., Mogilner, A., and Theriot, J. A. (2012). Choosing orientation: influence of cargo geometry and ActA polarization on actin comet tails. Molecular Biology of the Cell, 23(4):614-629.

Laub, M. and Loomis, W. (1998). A molecular network that produces spontaneous oscillations in excitable cells of Dictyostelium. Molecular Biology of the Cell, 9(12):35213532. 
Lee, S., Shen, Z., Robinson, D. N., Briggs, S., and Firtel, R. A. (2010). Involvement of the Cytoskeleton in Controlling Leading-Edge Function during Chemotaxis. Molecular Biology of the Cell, 21(11):1810-1824.

Loomis, W. (1971). Sensitivity of Dictyostelium-discoideum to nucleic acid analogues. Experimental Cell Research, 64(2):484-\&.

Lovelady, D. C., Richmond, T. C., Maggi, A. N., Lo, C. M., and Rabson, D. A. (2007). Distinguishing cancerous from noncancerous cells through analysis of electrical noise. Physical Review E, 76(4, Part 1).

Ma, H., Gamper, M., Parent, C., and Firtel, R. (1997). The Dictyostelium MAP kinase kinase DdMEK1 regulates chemotaxis and is essential for chemoattractant-mediated activation of guanylyl cyclase. EMBO Journal, 16(14):4317-4332.

Machesky, L. and Insall, R. (1998). Scar1 and the related Wiskott-Aldrich syndrome protein, WASP, regulate the actin cytoskeleton through the Arp2/3 complex. Current Biology, 8(25):1347-1356.

Machesky, L., Mullins, R., Higgs, H., Kaiser, D., Blanchoin, L., May, R., Hall, M., and Pollard, T. (1999). Scar, a WASp-related protein, activates nucleation of actin filaments by the Arp2/3 complex. Proceedings of the National Academy of Sciences of the United States of America, 96(7):3739-3744.

Marmarelis, P. (1978). Analysis of physiological systems. Plenum Press, New York, NY.

Maroto, M. and Monak, N. (2008). Cellular oscillatory mechanisms. Landes Bioscience, Austin, TX, USA.

Martiel, J. and Goldbeter, A. (1987). A model based on receptor desensitization for cyclicAMP signaling in Dictyostelium cells. Biophysical Journal, 52(5):807-828.

Martin, P., Bozovic, D., Choe, Y., and Hudspeth, A. (2003). Spontaneous oscillation by hair bundles of the bullfrog's sacculus. Journal of Neuroscience, 23(11):4533-4548.

McCray, J., Herbette, L., Kihara, T., and Trentham, D. (1980). A new approach to timeresolved studies of ATP-requiring biological-systems - laser flash-photolysis of caged ATP. Proceedings of the National Academy of Sciences of the United States of America - Biological Sciences, 77(12):7237-7241. 
Min, T. L., Mears, P. J., Golding, I., and Chemla, Y. R. (2012). Chemotactic adaptation kinetics of individual Escherichia coli cells. Proceedings of the National Academy of Sciences of the United States of America, 109(25):9869-9874.

Moreau, L. and Sontag, E. (2003). Balancing at the border of instability. Physical Review $E, 68(2$, Part 1).

Murray, J. (2003). Mathematical biology. Springer Science+Buisness Media.

Neilson, M. P., Veltman, D. M., van Haastert, P. J. M., Webb, S. D., Mackenzie, J. A., and Insall, R. H. (2011). Chemotaxis: A feedback-based computational model robustly predicts multiple aspects of real cell behaviour. PLOS Biology, 9(5).

Nerbonne, J., Richard, S., Nargeot, J., and Lester, H. (1984). New photoactivatable cyclicnucleotides produce intracellular jumps in cyclic-AMP and cyclic-GMP concentrations. Nature, 310(5972):74-76.

Neujahr, R., Albrecht, R., Kohler, J., Matzner, M., Schwartz, J., Westphal, M., and Gerisch, G. (1998). Microtubule-mediated centrosome motility and the positioning of cleavage furrows in multinucleate myosin II null cells. Journal of Cell Science, 111(Part 9):12271240.

Nicol, A., Rappel, W., Levine, H., and Loomis, W. (1999). Cell-sorting in aggregates of Dictyostelium discoideum. Journal of Cell Science, 112(22):3923-3929.

Norgauer, J., Krutmann, J., Dobos, G., Traynorkaplan, A., Oades, Z., and Schraufstatter, I. (1994). Actin polymerization, calcium-transients, and phospholipid-metabolism in human neutrophils after stimulation with interleukin-8 and n-formyl peptide. Journal of Investigative Dermatology, 102(3):310-314.

Okada, K., Obinata, T., and Abe, H. (1999). XAIP1: a Xenopus homologue of yeast actin interacting protein 1 (AIP1), which induces disassembly of actin filaments cooperatively with ADF cofilin family proteins. Journal of Cell Science, 112(10):1553-1565.

Omann, G., Porasik, M., and Sklar, L. (1989). Oscillating actin polymerization depolymerization responses in human polymorphonuclear leukocytes. Journal of Biological Chemistry, 264(28):16355-16358.

Pang, K., Lee, E., and Knecht, D. (1998). Use of a fusion protein between GFP and an anti-binding domain to visualize transient filamentous-actin structures. Current Biology, 8(7):405-408. 
Pantaloni, D., Carlier, M., Coue, M., Lal, A., Brenner, S., and Korn, E. (1984). The critical concentration of actin in the presence of ATP increases with the number concentration of filaments and approaches the critical concentration of actin.ADP. Journal of Biological Chemistry, 259(10):6274-6283.

Parent, C., Blacklock, B., Froehlich, W., Murphy, D., and Devreotes, P. (1998). G protein signaling events are activated at the leading edge of chemotactic cells. Cell, 95(1):81-91.

Parent, C. and Devreotes, P. (1996). Molecular genetics of signal transduction in Dictyostelium. Annual Review of Biochemistry, 65:411-440.

Park, K., Rivero, F., Meili, R., Lee, S., Apone, F., and Firtel, R. (2004). Rac regulation of chemotaxis and morphogenesis in Dictyostelium. EMBO Journal, 23(21):4177-4189.

Poff, K. and Butler, W. (1974). Absorbance changes induced by blue-light in Phycomycesblakesleeanus and Dictyostelium discoideum. Nature, 248(5451):798-801.

Poff, K., Loomis, W., and Butler, W. (1974). Isolation and purification of photoreceptor pigment associated with phototaxis in Dictyostelium-discoideum. Journal of Biological Chemistry, 249(7):2164-2167.

Pollard, T., Blanchoin, L., and Mullins, R. (2000). Molecular mechanisms controlling actin filament dynamics in nonmuscle cells. Annual Review of Biophysics and Biomolecular Structure, 29:545-576.

Pollard, T. and Borisy, G. (2003). Cellular motility driven by assembly and disassembly of actin filaments. Cell, 112(4):453-465.

Pollard, T. D. (2007). Regulation of actin filament assembly by Arp2/3 complex and formins. Annual Review of Biophysics and Biomolecular Structure, 36:451-477.

Pollard, T. D. and Cooper, J. A. (2009). Actin, a central player in cell shape and movement. Science, 326(5957):1208-1212.

Potts, G. (1902). Zur Physiologie des Dictyostelium mucoroides. Flora, 91:281-347.

Prassler, J., Murr, A., Stocker, S., Faix, J., Murphy, J., and Marriott, G. (1998). DdLIM is a cytoskeleton-associated protein involved in the protrusion of lamellipodia in Dictyostelium. Molecular Biology of the Cell, 9(3):545-559.

Qin, D., Xia, Y., and Whitesides, G. M. (2010). Soft lithography for micro- and nanoscale patterning. Nature Protocols, 5(3):491-502. 
Raper, K. (1935). Dictyostelium discoideum, a new species of slime mold from decaying forest leaves. Journal of Agricultural Research, 50:0135-0147.

Raper, K. (1937). Growth and development of Dictyostelium discoideum with different bacterial associates. Journal of Agricultural Research, 55:0289-0316.

Raper, K. and Smith, N. (1939). The growth of Dictyostelium discoideum upon pathogenic bacteria. Journal of Bacteriology, 38(4):431-445.

Rappel, W.-J. (2010). Personal communication.

Rappel, W.-J. and Loomis, W. F. (2009). Eukaryotic chemotaxis. WILEY Interdisciplinary Reviews-Systems Biology and Medicine, 1(1):141-149.

Riedl, J., Crevenna, A. H., Kessenbrock, K., Yu, J. H., Neukirchen, D., Bista, M., Bradke, F., Jenne, D., Holak, T. A., Werb, Z., Sixt, M., and Wedlich-Soldner, R. (2008). Lifeact: a versatile marker to visualize F-actin. Nature Methods, 5(7):605-607.

Rubel, E. and Cramer, K. (2002). Choosing axonal real estate: Location, location, location. Journal of Comparative Neurology, 448(1):1-5.

Sadler, I., Crawford, A., Michelsen, J., and Beckerle, M. (1992). Zyxin and CCRP - 2 interactive Lim domain proteins associated with the cytoskeleton. Journal of Cell Biology, 119(6):1573-1587.

Sakai, H. and Naka, K. (1987). Signal transmission in the catfish retina .4. Transmission to Ganglion-cells. Journal of Neurophysiology, 58(6):1307-1328.

Samadani, A., Mettetal, J., and van Oudenaarden, A. (2006). Cellular asymmetry and individuality in directional sensing. Proceedings of the National Academy of Sciences of the United States of America, 103(31):11549-11554.

Sandmann, R. (2009). Microfluidic study of eukaryotic chemotaxis: periodic stimulation and intracellular response. Master's thesis, Georg August University Goettingen.

Sasaki, A., Chun, C., Takeda, K., and Firtel, R. (2004). Localized Ras signaling at the leading edge regulates PI3K, cell polarity, and directional cell movement. Journal of Cell Biology, 167(3):505-518.

Sasaki, A. T., Janetopoulos, C., Lee, S., Charest, P. G., Takeda, K., Sundheimer, L. W., Meili, R., Devreotes, P. N., and Firtel, R. A. (2007). G protein-independent Ras/PI3K/Factin circuit regulates basic cell motility. Journal of Cell Biology, 178(2):185-191. 
Saucerman, J. J., Zhang, J., Martin, J. C., Peng, L. X., Stenbit, A. E., Tsien, R. Y., and McCulloch, A. D. (2006). Systems analysis of PKA-mediated phosphorylation gradients in live cardiac myocytes. Proceedings of the National Academy of Sciences of the United States of America, 103(34):12923-12928.

Schaefer, E., Tarantola, M., Polo, E., Westendorf, C., Oikawa, N., Bodenschatz, E., Geil, B., and Janshoff, A. (2012). Chemotaxis of d. discoideum cells on ultraelectrodes monitored by impedance analysis. submitted to PLOSone.

Schaefer, E., Westendorf, C., Bodenschatz, E., Beta, C., Geil, B., and Janshoff, A. (2011). Shape oscillations of Dictyostelium discoideum cells on ultramicroelectrodes monitored by impedance analysis. Small, 7(6):723-726.

Schneider, N., Weber, I., Faix, J., Prassler, J., Muller-Taubenberger, A., Kohler, J., Burghardt, E., Gerisch, G., and Marriott, G. (2003). A LIM protein involved in the progression of cytokinesis and regulation of the mitotic spindle. Cell Motility and the Cytoskeleton, 56(2):130-139.

Segall, J., Block, S., and Berg, H. (1986). Temporal comparisons in bacterial chemotaxis. Proceedings of the National Academy of Sciences of the United States of America, 83(23):8987-8991.

Servant, G., Weiner, O., Herzmark, P., Balla, T., Sedat, J., and Bourne, H. (2000). Polarization of chemoattractant receptor signaling during neutrophil chemotaxis. Science, 287(5455):1037-1040.

Shimizu, T. S., Tu, Y., and Berg, H. C. (2010). A modular gradient-sensing network for chemotaxis in Escherichia coli revealed by responses to time-varying stimuli. Molecular Systems Biology, 6.

Skoge, M., Adler, M., Groisman, A., Levine, H., Loomis, W. F., and Rappel, W.-J. (2010). Gradient sensing in defined chemotactic fields. Integrative Biology, 2(11-12):659-668.

Spoon, D. (1978). New rotary microcompressor. Transactions of the American Microscopical Society, 97(3):412-416.

Squires, T. and Quake, S. (2005). Microfluidics: Fluid physics at the nanoliter scale. Reviews of Modern Physics, 77(3):977-1026.

Steffen, A., Rottner, K., Ehinger, J., Innocenti, M., Scita, G., Wehland, J., and Stradal, T. (2004). Sra-1 and Nap1 link Rac to actin assembly driving lamellipodia formation. EMBO Journal, 23(4):749-759. 
Strogatz, S. H. (2000). Nonlinear dynamics and chaos. Perseus book publishing, New York City, NY, USA.

Struenker, T., Goodwin, N., Brenker, C., Kashikar, N. D., Weyand, I., Seifert, R., and Kaupp, U. B. (2011). The CatSper channel mediates progesterone-induced Ca2+ influx in human sperm. Nature, 471(7338):382+.

Sussman, R. and Sussman, M. (1967). Cultivation of Dictyostelium discoideum in axenic medium. Biochemical and Biophysical Research Communications, 29(1):53-\&.

Swaney, K. F., Huang, C.-H., and Devreotes, P. N. (2010). Eukaryotic Chemotaxis: A network of signaling pathways controls motility, directional sensing, and polarity. Annual Review of Biophysics, 39:265-289.

Takeda, K., Shao, D., Adler, M., Charest, P. G., Loomis, W. F., Levine, H., Groisman, A., Rappel, W.-J., and Firtel, R. A. (2012). Incoherent Feedforward Control Governs Adaptation of Activated Ras in a Eukaryotic Chemotaxis Pathway. Science Signaling, $5(205)$.

Taylor, G. (1953). Dispersion of soluble matter in solvent flowing slowly through a tube. Proceedings of the Royal Society of London Series A-Mathematical and Physical Sciences, 219(1137):186-203.

Tero, A., Takagi, S., Saigusa, T., Ito, K., Bebber, D. P., Fricker, M. D., Yumiki, K., Kobayashi, R., and Nakagaki, T. (2010). Rules for biologically inspired adaptive network design. Science, 327(5964):439-442.

Theves, M. (2009). Quantitative study of eukaryotic chemotaxis with microfluidic devices. Master's thesis, Georg August University Goettingen, Germany.

Thornhill, N., Huang, B., and Zhang, H. (2003). Detection of multiple oscillations in control loops. Journal of Process Control, 13(1):91-100.

Tomchik, K. and Devreotes, P. (1981). Adenosine-3',5'-monophosphate waves in Dictyostelium-discoideum - a demonstration by isotope dilution-fluorography. Science, 212(4493):443-446.

Tsien, R. (1998). The green fluorescent protein. Annual Review of Biochemistry, 67:509544. 
Tu, Y., Shimizu, T. S., and Berg, H. C. (2008). Modeling the chemotactic response of Escherichia coli to time-varying stimuli. Proceedings of the National Academy of Sciences of the United States of America, 105(39):14855-14860.

Uchida, K. and Yumura, S. (2004). Dynamics of novel feet of Dictyostelium cells during migration. Journal of Cell Science, 117(8):1443-1455.

Ueda, M., Sako, Y., Tanaka, T., Devreotes, P., and Yanagida, T. (2001). Single-molecule analysis of chemotactic signaling in Dictyostelium cells. Science, 294(5543):864-867.

Uhlig, G. and Heimberg, S. (1981). A new versatile compression chamber for examination of living microorganisms. Helgolander Meeresuntersuchungen, 34(3):251-256.

Van Haastert, P. (1987). Differential effects of temperature on cyclic amp-induced excitation adaptation and deadaptation of adenylate and guanylate cyclase in dictyosteliumdiscoideum. Journal of Cell Biology, 105(5):2301-2306.

Van Haastert, P. J. M. (2010). Chemotaxis: insights from the extending pseudopod. Journal of Cell Science, 123(18):3031-3037.

Veltman, D. M. and Insall, R. H. (2010). WASP family proteins: Their evolution and its physiological implications. Molecular Biology of the Cell, 21(16):2880-2893.

Veltman, D. M., Keizer-Gunnik, I., and Van Haastert, P. J. M. (2008). Four key signaling pathways mediating chemotaxis in Dictyostelium discoideum. Journal of Cell Biology, 180(4):747-753.

Veltman, D. M. and van Haastert, P. J. M. (2008). The role of cGMP and the rear of the cell in Dictyostelium chemotaxis and cell streaming. Journal of Cell Science, 121(1):120 127.

Vicker, M. (2002). Eukaryotic cell locomotion depends on the propagation of self-organized reaction-diffusion waves and oscillations of actin filament assembly. Experimental Cell Research, 275(1):54-66.

Vicker, M. G. and Grutsch, J. F. (2008). Dual chemotaxis signalling regulates Dictyostelium development: Intercellular cyclic AMP pulses and intracellular F-actin disassembly waves induce each other. European Journal of Cell Biology, 87(10):845-861.

Wang, C. J., Bergmann, A., Lin, B., Kim, K., and Levchenko, A. (2012). Diverse sensitivity thresholds in dynamic signaling responses by social amoebae. Science Signaling, 5(213). 
Watts, D. and Ashworth, J. (1970). Growth of myxamoebae of cellular slime mould Dictyostelium-discoideum in axenic culture. Biochemical Journal, 119(2):171-\&.

Webb, D., Donais, K., Whitmore, L., Thomas, S., Turner, C., Parsons, J., and Horwitz, A. (2004). FAK-Src signalling through paxillin, ERK and MLCK regulates adhesion disassembly. Nature Cell Biology, 6(2):154+.

Weiss, J., Karma, A., Shiferaw, Y., Chen, P., Garfinkel, A., and Qu, Z. (2006). From pulsus to Pulseless the saga of cardiac alternans. Circulation Research, 98(10):1244-1253.

Welch, M. and Mullins, R. (2002). Cellular control of actin nucleation. Annual Review of Cell and Developmental Biology, 18:247-288.

Westendorf, C., Bae, A., Erlenkamper, C., Galland, E., Franck, C., Bodenschatz, E., and Beta, C. (2010). Live cell flattening-traditional and novel approaches. PMC Biophysics, page $15 \mathrm{pp}$.

Westendorf, C., Negrete Jr., J., Bae, A., Sandmann, R., Bodenschatz, E., and Beta, C. (submitted 09 / 2012). The actin cytoskeleton of chemotactic amoeba operates close to the onset of oscillations. submitted to PNAS.

Whitesides, G., Ostuni, E., Takayama, S., Jiang, X., and Ingber, D. (2001). Soft lithography in biology and biochemistry. Annual Review of Biomedical Engineering, 3:335-373.

Williams, K., Kessin, R., and Newell, P. (1974). Genetics of growth in axenic medium of cellular slime-mold Dictyostelium-discoideum. Nature, 247(5437):142-143.

Wymann, M., Kernen, P., Bengtsson, T., Andersson, T., Baggiolini, M., and Deranleau, D. (1990). Corresponding oscillations in neutrophil shape and filamentous actin content. Journal of Biological Chemistry, 265(2):619-622.

Xia, Y. and Whitesides, G. (1998). Soft lithography. Angewandte Chemie-International Edition, 37(5):551-575.

Xiao, Z., Zhang, N., Murphy, D., and Devreotes, P. (1997). Dynamic distribution of chemoattractant receptors in living cells during chemotaxis and persistent stimulation. Journal of Cell Biology, 139(2):365-374.

Yumura, S. (1994). Rapid translocation of myosin-ii in vegetative Dictyostelium amebas during chemotactic stimulation by Folic-acid. Cell Structure and Function, 19(3):143151. 
Yumura, S. and Fukui, Y. (1985). Reversible cyclic AMP-dependent change in distribution of myosin thick filaments in Dictyostelium. Nature, 314(6007):194-196.

Yumura, S., Mori, H., and Fukui, Y. (1984). Localization of Actin and Myosin for the study of ameboid movement in Dictyostelium using improved immunofluorescence. Journal of Cell Biology, 99(3):894-899. 


\title{
Curriculum Vitae
}

\author{
Personal Data
}

Name: Christian Westendorf.

Date of birth: May 17, 1980.

Place of birth: Rostock, Germany.

Nationality: German.

\section{Education and work experience}

05/2007-

PhD thesis on the oscillatory dynamics of the actin cytoskeleton.

MPI for Dynamics and Self-Organization, Göttingen, Germany.

Supervisors: Prof. Dr. E. Bodenschatz and Prof. Dr. C. Beta.

05/2007-

PhD in physics.

Georg-August University, Göttingen, Germany.

Major subject: Biophysics.

Minor subjects: Astrophysics, nonlinear dynamics.

03/2007-04/2007 Research assistant in technical chemistry.

Department of Technical Chemistry, University of Rostock, Germany.

Supervisor: Prof. Dr. U. Kragl.

07/2005-02/2006 Diploma thesis on plant/microbe interaction.

University of Rostock, Germany and Technical University of Graz, Austria.

Supervisor: Prof. Dr. G. Berg.

10/1999-09/2006 Diploma studies in biology (graded 1.1).

Major subject: Microbiology.

University of Rostock, Germany.

$07 / 1998$

German university entrance qualification (graded 1.5).

Richard-Wossidlo-Gymnasium, Ribnitz-Damgarten, Germany. 
Internship and Training

08/2007-10/2007 EU training program "Non-equilibrium in Physics and in Biology", St.Etienne de Tinée, France.

10/2005-11/2005 Intern at the department of microbiology of the university Zurich, Switzerland.

\section{Publications}

submit. 09/2012 "The actin cytoskeleton of chemotactic amoebae operates close to the onset of oscillations".

C. Westendorf, J. Negrete Jr., A. Bae, R. Sandmann, E. Bodenschatz and C. Beta, submitted to PNAS.

submit. 08/2012 "Chemotaxis of D. discoideum: collective oscillation of cellular contacts".

E. Schaefer, M. Tarantola, E. Polo, C. Westendorf, N. Oikawa, E. Bodenschatz, B. Geil and A. Janshoff, submitted to PLOS ONE.

$01 / 2011$

"Shape oscillations of Dictyostelium discoideum cells on ultraelectrodes monitored by impedance analysis".

E. Schaefer, C. Westendorf, E. Bodenschatz, C. Beta, B. Geil and A. Jahnshoff, Small (2011), 7(6), 723-726.

04/2010 "Live cell flattening - traditional and novel approaches".

C. Westendorf, A. Bae, C. Erlenkamper, E. Galland, C. Franck, E. Bodenschatz and C. Beta, PMC Biophysics (2010) 3:9.

11/2009 "The Caenorhabditis elegans assay: a tool to evaluate the pathogenic potential of bacterial biocontrol agents".

C. Zachow, H. Pirker, C. Westendorf, R. Tilcher and G. Berg, Eur J Plant Pathol (2009) 125:367-376.

$3 / 2009$

"Quorum- sensing effects in the antagonistic rhizosphere bacterium Serratia plymuthica HRO- C48".

H. Mueller, C. Westendorf, E. Leitner, L. Chernin, K. Riedel, S. Schmidt, L. Eberl and G. Berg, FEMS Microbiol Ecol (2009) 67 468-478c. 
$09 / 2012$

$07 / 2011$

$08 / 2010$

$03 / 2009$
Annual meeting of the German Biophysical Society 2012.

"Oscillatory instabilities of intracellular fiber networks" (Poster).

Georg-August University, Göttingen, Germany.

Scientific advisory board 2011.

"Biophysics of cellular dynamics" (Talk).

MPI for Dynamics and Self-Organization, Göttingen, Germany.

International Dictyostelium meeting 2010.

"Response of the actin cytoskeleton to rapidly applied stimuli" (Poster). Cardiff University, Cardiff, Wales.

German physical society (DPG) spring meeting 2009.

"Dynamics of the actin cytoskeleton in response to periodic stimuli" (Talk).

Technical University of Dresden, Germany.

\section{Teaching Experience}

04/2010-07/2012 Organisation of the seminar Current topics in biophysics and cell biology (together with E. Bodenschatz).

03/2010 Teaching assistant for the graduate lab course Confocal laser scanning microscopy.

04/2009-08/2009 Teaching assistant for the lecture Biophysics and physics of complex systems.

04/2008-02/2009 Teaching assistant for the lab course in mechanics, thermodynamics and optics for students with physics as a minor subject.

10/2007-02/2008 Teaching assistant for the lecture Biophysics II.

Göttingen, September 27, 2012 